\title{
Duas abordagens para casamento de padrões de pontos usando relações espaciais e casamento entre grafos
}

\author{
Alexandre Noma \\ TESE APRESENTADA \\ $\mathrm{AO}$ \\ Instituto De Matemática e Estatística \\ DA \\ Universidade de São Paulo \\ PARA OBTENÇÃO DO GRAU DE DOUTOR \\ EM \\ Ciência da Computação
}

Orientador: Prof. Dr. Roberto Marcondes Cesar Junior.

— São Paulo, Julho de 2010 -

- Durante este trabalho, o aluno recebeu apoio financeiro da FAPESP- 



\title{
Duas abordagens para casamento de padrões de pontos usando relações espaciais e casamento entre grafos
}

\author{
Este exemplar corresponde à redação final \\ da tese devidamente corrigida e \\ defendida por Alexandre Noma \\ e aprovada pela comissão julgadora.
}

São Paulo, 15 de julho de 2010

Banca examinadora:

- Prof. Dr. Roberto Marcondes Cesar Junior - IME-USP

- Prof. Dr. Anderson de Rezende Rocha - UNICAMP

- Profa. Dra. Leila Maria Garcia Fonseca - INPE

- Profa. Dra. Maria Cristina Ferreira de Oliveira - ICMC-USP

- Profa. Dra. Nina Sumiko Tomita Hirata - IME-USP 

Aos meus pais Massagi, Eliana, Luiz e Yoshiko. Aos meus irmãos Eduardo, Beatriz, Leandro e Larissa. À Ayumi, com muito amor. 



\section{Agradecimentos}

Primeiramente, meus sinceros agradecimentos ao professor Roberto Marcondes Cesar Junior, pela oportunidade, confiança, dedicação, paciência e compreensão.

Ao professor Luís Augusto Consularo, pelo suporte e pela disponibilização do código fonte do seu programa de segmentação interativa de imagens, proporcionando o ponto de partida para as implementações dos algoritmos deste trabalho.

Ao professor Alvaro Pardo, pela dedicação, agilidade e inteligência, tendo um especial destaque em promover um trabalho em equipe, sendo o principal responsável pelas nossas primeiras contribuições concretas em congresso e em revista.

Ao professor Luiz Velho, por fonecer uma aplicação inteligente e divertida para as nossas contribuições.

Aos professores e funcionários do IME-USP, especialmente aos meus orientadores de mestrado, Cris e Coelho, e aos funcionários da seção de pós-graduação e do audio-visual.

A todos os colegas, especialmente do laboratório da Rede Vision do IME-USP, pela amizade e ajuda. Dentre eles, não posso deixar de mencionar DavidJr, M.Oikawa, Jesús, DavidSP, Mh, Th, Paixão, Klava, ABVG, Celina, Jihan, Talita, Wonder, Pedro, DDantas, Paschoal, André e Fabrício.

À FAPESP pelo apoio financeiro.

Finalmente, sou eternamente grato aos meus pais, irmãos, familiares, e especialmente à minha esposa. Esta tese é integralmente dedicada a eles. 



\section{Resumo}

Casamento de padrões de pontos é um problema fundamental em reconhecimento de padrões. O objetivo é encontrar uma correspondência entre dois conjuntos de pontos, associados a características relevantes de objetos ou entidades, mapeando os pontos de um conjunto no outro. Este problema está associado a muitas aplicações, como por exemplo, reconhecimento de objetos baseado em modelos, imagens estéreo, registro de imagens, biometria, entre outros. Para encontrar um mapeamento, os objetos são codificados por representações abstratas, codificando as características relevantes consideradas na comparação entre pares de objetos. Neste trabalho, objetos são representados por grafos, codificando tanto as características 'locais' quanto as relações espaciais entre estas características. A comparação entre objetos é guiada por uma formulação de atribuição quadrática, que é um problema NP-difícil. Para estimar uma solução, duas técnicas de casamento entre grafos são propostas: uma baseada em grafos auxiliares, chamados de grafos deformados; e outra baseada em representações 'esparsas', campos aleatórios de Markov e propagação de crenças. Devido as suas respectivas limitações, as abordagens são adequadas para situações específicas, conforme mostrado neste documento. Resultados envolvendo as duas abordagens são ilustrados em quatro importantes aplicações: casamento de imagens de gel eletroforese 2D, segmentação interativa de imagens naturais, casamento de formas, e colorização assistida por computador. 



\begin{abstract}
Point set matching is a fundamental problem in pattern recognition. The goal is to match two sets of points, associated to relevant features of objects or entities, by finding a mapping, or a correspondence, from one set to another set of points. This issue arises in many applications, e.g. model-based object recognition, stereo matching, image registration, biometrics, among others. In order to find a mapping, the objects can be encoded by abstract representations, carrying relevant features which are taken into account to compare pairs of objects. In this work, graphs are adopted to represent the objects, encoding their 'local' features and the spatial relations between these features. The comparison of two given objects is guided by a quadratic assignment formulation, which is NP-hard. In order to estimate the optimal solution, two approximations techniques, via graph matching, are proposed: one is based on auxiliary graphs, called deformed graphs; the other is based on 'sparse' representations, Markov random fields and belief propagation. Due to their respective limitations, each approach is more suitable to each specific situation, as shown in this document. The quality of the two approaches is illustrated on four important applications: 2D electrophoresis gel matching, interactive natural image segmentation, shape matching, and computer-assisted colorization.
\end{abstract}





\section{Sumário}

Lista de Abreviaturas e Termos $\quad$ v

Lista de Símbolos vii

$\begin{array}{ll}\text { Lista de Figuras } & \text { ix }\end{array}$

1 Introdução 1

1.1 Motivação . . . . . . . . . . . . . . . . . . . 1

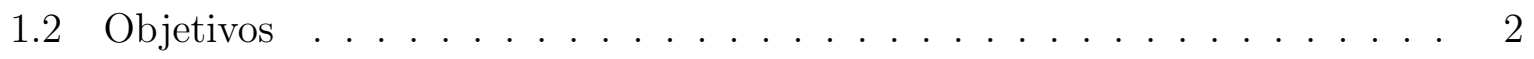

1.3 Contribuições . . . . . . . . . . . . . . . . . . . . . 3

1.4 Organização . . . . . . . . . . . . . . . . . . 4

2 Casamento de padrões de pontos via casamento entre grafos 5

2.1 Casamento entre grafos . . . . . . . . . . . . . . . . . 5

2.1 .1 Exato vs Inexato . . . . . . . . . . . . . . . 6

2.1.2 Homomorfismo vs Isomorfismo . . . . . . . . . . . . . 6

2.1.3 Histórico . . . . . . . . . . . . . . . . . . . . . . . . . . 7

2.2 Formulação via atribuição quadrática . . . . . . . . . . . . . . . . . . . . 10

2.2.1 Termo linear: distância de aparência $\left(d_{A}\right) \ldots \ldots$. . . . . . . 11

2.2.2 Termo quadrático: distância estrutural $\left(d_{S}\right) \ldots \ldots$. . . . . . . . 11

2.2.3 Desafio em casamento entre grafos . . . . . . . . . . . . 12 
2.3 Casamento via grafos deformados . . . . . . . . . . . . . . . . . . 12

2.3.1 Grafos deformados (DGs) . . . . . . . . . . . . . . . . 12

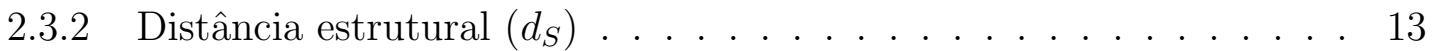

2.3.3 Otimização . . . . . . . . . . . . . . . . . . 15

2.4 Aplicação 1: casamento entre gels de eletroforese 2D . . . . . . . . . . . . . 17

2.4.1 Histórico . . . . . . . . . . . . . . . . . . . . . . . . . 18

2.4.2 Detecção de proteínas . . . . . . . . . . . . . . 20

2.4.3 Distância entre shape context . . . . . . . . . . . . . . . 20

2.4.4 Experimentos . . . . . . . . . . . . . . . . . . 21

2.5 Aplicação 2: segmentação interativa de imagens naturais . . . . . . . . . . 33

2.5.1 Histórico . . . . . . . . . . . . . . . . . . . 34

2.5.2 Abordagem de segmentação interativa . . . . . . . . . . . . . 35

2.5.3 Distância entre cores . . . . . . . . . . . . . . . . . 37

2.5.4 Pós-processamento . . . . . . . . . . . . . . . . . 38

2.5.5 Experimentos ....................... 40

2.6 Resumo da abordagem DG . . . . . . . . . . . . . . . . . 57

3 Casamento de padrões de pontos via campos aleatórios de Markov 59

3.1 Campos aleatórios de Markov . . . . . . . . . . . . . . . . . 59

3.1.1 Ingredientes principais de um MRF . . . . . . . . . . . . 60

3.1.2 Casamento entre grafos via MRFs . . . . . . . . . . . . . 61

3.1.3 MRFs em termos de campos aleatórios de Gibbs . . . . . . . . . . . 62

3.2 Formulação via máxima a posteriori . . . . . . . . . . . . . . . . . 63

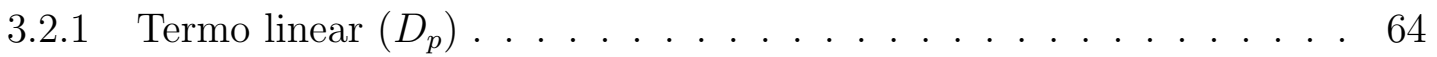

3.2.2 Termo quadrático: Markov $(M) \ldots \ldots . \ldots . \ldots 65$

3.3 Casamento via propagação de crenças $(\mathrm{BP})$. . . . . . . . . . . . . 65 
3.3 .1 Termo de Markov . . . . . . . . . . . . . . . . . . . 66

3.3 .2 Otimização eficiente via mínima-convolução . . . . . . . . . . . 67

3.4 Aplicação 1: casamento entre formas . . . . . . . . . . . . . . . . . . 69

3.4 .1 Histórico . . . . . . . . . . . . . . . . . . . . . 70

3.4 .2 Representação esparsa para as formas . . . . . . . . . . . . . . 71

3.4 .3 Distância entre formas . . . . . . . . . . . . . . . 71

3.4.4 Experimentos usando silhuetas . . . . . . . . . . . . . . . 72

3.4.5 Experimentos usando MNIST e COIL . . . . . . . . . . . 77

3.5 Aplicação 2: colorização assistida por computador . . . . . . . . . . . . . . 91

3.5 .1 Histórico . . . . . . . . . . . . . . . . . . . . . . . . . . 92

3.5 .2 Abordagem de colorização . . . . . . . . . . . . . . . . . 93

3.5.3 Distância entre regiões . . . . . . . . . . . . . . . . . . 94

3.5 .4 Experimentos . . . . . . . . . . . . . . . . . . 94

3.6 Resumo da abordagem BP . . . . . . . . . . . . . . . . . . . . . . . 104

4 Conclusões 105

4.1 Comentários finais . . . . . . . . . . . . . . . . . . . . 105

4.2 Trabalhos futuros . . . . . . . . . . . . . . . . . . . 107 


\section{Lista de Abreviaturas e Termos}

2DE eletroforese bidimensional

2DEM casamento entre gels 2DE

ARG grafo relacional com atributos

BGM casamento de grafos bipartidos

BP propagação de crenças

CAC colorização assistida por computador

CIELAB espaço de cores CIE $1976\left(L^{*}, a^{*}, b^{*}\right)$

DG grafo deformado

GA graduated assignment

GC graph cuts

GRF campo aleatório de Gibbs

ICP iterative closest point

INIS segmentação interativa de imagens naturais

MAP máxima a posteriori

MCS subgrafo comum máximo

MRF campo aleatório de Markov

SM casamento entre formas ou shape matching

$\mathrm{SC} \quad$ shape context

TPS thin-plate splines 


\section{Lista de Símbolos}

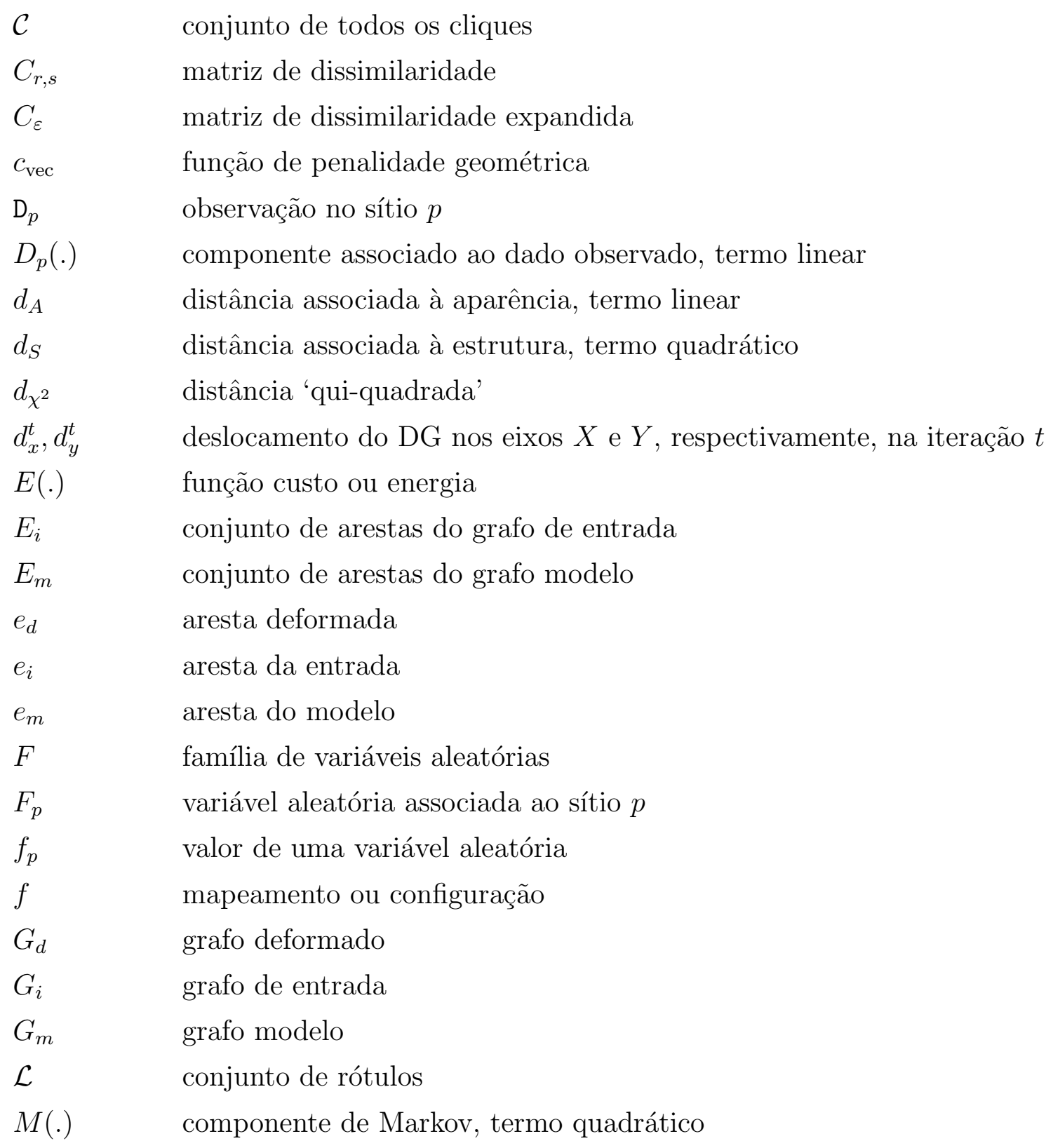




$\begin{array}{ll}m_{p q}^{t} & \text { mensagem BP do sítio } p \text { ao sítio } q \text {, na iteração } t \\ \mathcal{N} & \text { sistema de vizinhança } \\ P(.), p(.) & \text { distribuição de probabilidade } \\ p, q \in V_{i} & \text { vértices da entrada } \\ Q_{r, s} & \text { matriz de permutação } \\ \mathcal{S} & \text { conjunto de sítios } \\ \mathrm{SC}(.) & \text { atributos de shape context } \\ V_{c}(.) & \text { função potencial de clique } \\ V_{i} & \text { conjunto de vértices da entrada } \\ V_{m} & \text { conjunto de vértices do modelo } \\ v_{d} & \text { vértice deformado } \\ v_{i} & \text { vértice da entrada } \\ v_{m} & \text { vértice do modelo } \\ \alpha, \beta \in V_{m} & \text { vértices do modelo } \\ \varepsilon & \text { limiar para BGM usando o método Húngaro } \\ \lambda_{1} & \text { parâmetro para balancear os termos, linear e quadrático, de } E(.) \\ \lambda_{2} & \text { parâmetro para balancear os termos, angular e modular, de } c_{\text {vec }} \\ \mu & \text { atributos de vértices } \\ \nu & \text { atributos de arestas } \\ \theta & \text { ângulo entre dois vetores }\end{array}$




\section{Lista de Figuras}

1.1 Dois padrões de pontos. Os pontos correspondentes estão conectados por linhas pontilhadas. . . . . . . . . . . . . . . . . 1

2.1 (a) Dois padrões de pontos, usados como modelo e entrada, respectivamente. (b) Informação estrutural extraída do modelo, à esquerda, e sobreposto na entrada, à direita. (c) Grafo modelo $G_{m}$ e grafo deformado $G_{d}$ devido ao par $\left(v_{i}, v_{m}\right)$, respectivamente. $\ldots \ldots \ldots \ldots \ldots$

2.2 Correspondência entre um par de imagens 2DE. . . . . . . . . . . . 18

2.3 Detecção de proteínas. Curvas em verde ilustram bordas significativas. Pontos em vermelho indicam as posições com maiores concentrações protéicas. 20

2.4 Experimento 1. Comparação entre nosso algoritmo DG e BGM usando o método Húngaro, para diferentes valores de $\lambda_{1}$ e $\varepsilon$, respectivamente. Resultados obtidos de acordo com (a) diferentes percentuais de pontos removidos e (b) diferentes graus de ruído Gaussiano. . . . . . . . . . . . . . . . 22

2.5 Comparação entre nossa abordagem DG, BGM, BGM+TPS e GA para (a) diferentes porcentagens de pontos removidos e (b) diferentes graus de ruído Gaussiano. . . . . . . . . . . . . . . . . . . 23 
2.6 Comparação, usando (a) par complexo "095-098": grande quantidade de outliers e fortes deformações não-rígidas. O modelo se encontra à esquerda com $\left|V_{m}\right|=95$. Ao centro, a entrada possui $\left|V_{i}\right|=119$. Neste cenário complexo, existem 57 pontos da entrada sem correspondência. Na extrema direita, um exemplo de ruído Gaussiano $(\sigma=5)$ aplicado nos dois conjuntos de pontos, sobrepostos para uma melhor vizualização. (b) Comparação entre as abordagens DG, BGM, BGM+TPS, GA, considerando 100 pares artificiais obtidos de acordo com diferentes porcentagens de pontos removidos e diferentes valores de desvio padrão para ruído Gaussiano. A curva DG2 representa o algoritmo proposto simulando o conhecimento a priori fornecido pelo usuário. . . . . . . . . . . . . . . . . . . . 26

2.7 Comportamento médio dos métodos avaliados, usando 4500 pares artificiais, obtidos a partir de 45 pares originais, usando os marcadores de [2]. . .

2.8 Par "031-032": um exemplo em que nossa abordagem DG supera o algoritmo BGM+TPS. 1a. linha: para valores pequenos de $\epsilon$, um número excessivo de pontos da entrada são incorretamente descartados como ouliters pelo método BGM+TPS. Os ouliters são representados pelos números em destaque. 2a. linha: para altos valores de $\epsilon$, BGM+TPS produz correspondências incorretas, indicadas pelas linhas destacadas, sugerindo que a forma fechada de TPS é inadequada para este caso. 3a. linha: nosso resultado. . . . . . . . . . . . . . . . . . . 28

2.9 Nossos resultados para os pares "006-007", "008-009", "008-009". . . . . . 30

2.10 Nossos resultados para os pares "014-015", "016-017", "019-020" . . . . . 31

2.11 Nossos resultados para os pares "028-029", "074-075", "095-098" . . . . . 32

2.12 Resumo de nossa abordagem. Primeiramente, dividimos a imagem de entrada em regiões, via watershed. A seguir, dois padrões de pontos são gerados, um incluindo todas as regiões, e outro incluindo somente os pontos representando as regiões marcadas pelo usuário. Finalmente, o resultado da segmentação é obtido através do casamento entre os padrões de pontos. 
2.13 (a) Resultado obtido com dois traços, incorretamente incluindo parte do fundo. (b) Adição de um terceiro traço para eliminar completamente o fundo, corrigindo a extração das flores. Os contornos das regiões obtidas estão destacados. . . . . . . . . . . . . . . . . . . . . 36

2.14 Exemplos de segmentação usando imagens de Grabcut [76], Bai e Sapiro [7], e o 'tucano' usado em [62]. Coluna esquerda: imagens originais com traços do usuário. Coluna do meio: regiões rotuladas, sem o pós-processamento. Coluna direita: resultado após o pós-processamento.

2.15 Elementos estruturantes usados para suavizar as bordas do objeto de interesse.

2.16 Diferentes estilos de traços, com resultados similares de segmentação. .. . 40

2.17 Exemplos de segmentação usando imagens de: Berkeley [59], Grabcut [76], Bai e Sapiro [7]. Coluna esquerda: imagens originais com traços do usuário. Coluna do meio: regiões rotuladas. Coluna direita: composições de imagens com um novo fundo.

2.18 Banco de imagens de Berkeley [59]. Coluna esquerda: imagens originais com traços do usuário. Coluna do meio: regiões rotuladas. Coluna direita: objetos extraídos.

2.19 Banco de imagens Grabcut [76]. Coluna esquerda: imagens originais com traços do usuário. Coluna do meio: regiões rotuladas. Coluna direita: objetos extraídos.

2.20 Resultados quantitativos usando gabaritos de [60] e imagens de Berkeley [59].

2.21 Resultados quantitativos usando gabaritos de [60] e imagens de Berkeley [59].

2.22 Resultados quantitativos usando gabaritos de [60] e imagens de Berkeley [59].

2.23 Resultados quantitativos usando gabaritos de [60] e imagens de Berkeley [59].

2.24 Resultados quantitativos usando imagens de Grabcut [76]. . . . . . . . . . 48 
2.25 Resultados quantitativos usando imagens de Grabcut [76]. . . . . . . . . . 49

2.26 Resultados quantitativos usando imagens de Grabcut [76]. . . . . . . . . . 50

2.27 Resultados quantitativos usando imagens de Grabcut [76]. . . . . . . . . . 51

2.28 Exemplos adicionais de segmentação. Coluna esquerda: imagens originais com traços do usuário. Coluna do meio: regiões rotuladas. Coluna direita: composições de imagens com um novo fundo. . . . . . . . . . . . . . . 52

2.29 Exemplo de segmentação interativa com múltiplos rótulos. Coluna esquerda: Imagem original com traços do usuário. Coluna direita: regiões rotuladas. . . . . . . . . . . . . . . . . . . . 52

2.30 Comparação qualitativa entre seeded region growing (SRG) [3], simple interactive object extraction (SIOX) [41], interactive graph cuts (IGC) [17], e o nosso método DG. . . . . . . . . . . . . . . . 56

2.31 Imagem original e a respectiva máscara usada no papel dos traços do

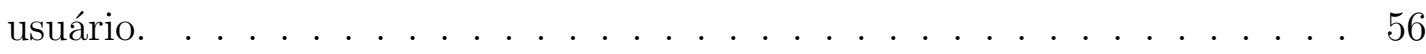

3.1 Resumo de nossa abordagem para casamento entre formas. . . . . . . . . 69

3.2 (a) Objeto. (b) Bordas obtidas pelo detector Canny. (c) Grafo esparso representando $(\mathrm{b}) . \ldots \ldots \ldots \ldots$. . . . . . . . . . . . . . . . . .

3.3 Exemplos de SM usando letras com diferentes fontes, dígitos manuscritos de MNIST [52], silhueta humana de [48] e silhuetas de Kimia [82]. (a) Silhueta de entrada. (b) Grafo de entrada. (c) Silhueta do modelo. (d) Grafo modelo. (e) Resultado de casamento usando nossa abordagem baseada em

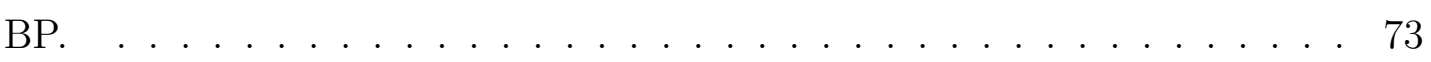

3.4 Exemplos de SM usando silhuetas de Kimia [82]. (a) Silhueta de entrada. (b) Grafo de entrada. (c) Silhueta do modelo. (d) Grafo modelo. (e) Resultado de casamento usando nossa abordagem baseada em BP. . . . . . . 74

3.5 Exemplos de SM comparando nossa abordagem baseada em BP, o método Húngaro (BGM), e graduated assignment (GA) [29]. . . . . . . . . . . 75

3.6 Em geral, variar $\varepsilon$ não resulta em eliminação dos cruzamentos de arestas pelo método BGM. . . . . . . . . . . . . . . . . . . . . . 75 
3.7 Um exemplo onde o método baseado em BP encontra corretamente o modelo $G_{m}$ imerso na entrada $G_{i}$. Por outro lado, GA tenta transformar todos os pontos, incluindo outliers, produzindo um resultado insatisfatório. . . . 76

3.8 Um exemplo de clutter, onde a entrada representa uma versão bastante ruidosa do modelo. Nosso resultado foi bastante satisfatório, em contraste com o resultado pobre obtido por GA . . . . . . . . . . . . . . 76

3.9 Taxas de erros para o reconhecimento de objetos 3D, usando o classificador 1-NN para diferentes tamanhos de conjuntos de treinamento. . . . . . . . 78

3.10 Usando 158 protótipos do banco de imagens COIL [61], nossa abordagem BP produziu 20 erros de um total de 1242 classificações.

3.11 Usando 158 protótipos do banco de imagens COIL [61], nossa abordagem BP produziu 20 erros de um total de 1242 classificações. . . . . . . . . . . . 80

3.12 Usando 158 protótipos do banco de imagens COIL [61], nossa abordagem BP produziu 20 erros de um total de 1242 classificações. . . . . . . . . . . 81

3.13 Usando 158 protótipos do banco de imagens COIL [61], nossa abordagem BP produziu 20 erros de um total de 1242 classificações. . . . . . . . . . . 82

3.14 Classificações incorretas produzidas pelo nosso classificador 1-NN, usando o banco de imagens COIL [61]. (a) Image de entrada. (b) Vizinho mais próximo, de acordo com a distância definida na Equação 3.22. (c) Grafo esparso representando (a), conforme descrito na Seção 3.4.2. (d) Similarmente, grafo esparso representando (b). (e) Casamento entre formas, onde os vértices de entrada sem correspondência estão destacados. . . . . . . . . 83

3.15 Classificações incorretas produzidas pelo nosso classificador 1-NN, usando o banco de imagens COIL [61]. (a) Image de entrada. (b) Vizinho mais próximo, de acordo com a distância definida na Equação 3.22. (c) Grafo esparso representando (a), conforme descrito na Seção 3.4.2. (d) Similarmente, grafo esparso representando (b). (e) Casamento entre formas, onde os vértices de entrada sem correspondência estão destacados. . . . . . . . 84 
3.16 Classificações incorretas produzidas pelo nosso classificador 1-NN, usando o banco de imagens COIL [61]. (a) Image de entrada. (b) Vizinho mais próximo, de acordo com a distância definida na Equação 3.22. (c) Grafo esparso representando (a), conforme descrito na Seção 3.4.2. (d) Similarmente, grafo esparso representando (b). (e) Casamento entre formas, onde os vértices de entrada sem correspondência estão destacados. . . . . . . . 85

3.17 Classificações incorretas produzidas pelo nosso classificador 1-NN, usando o banco de imagens COIL [61]. (a) Image de entrada. (b) Vizinho mais próximo, de acordo com a distância definida na Equação 3.22. (c) Grafo esparso representando (a), conforme descrito na Seção 3.4.2. (d) Similarmente, grafo esparso representando (b). (e) Casamento entre formas, onde os vértices de entrada sem correspondência estão destacados. . . . . . . . 86

3.18 Taxas de erros para o reconhecimento de dígitos manuscritos, usando classificadores $K$-NN para diferentes valores de $K \ldots \ldots \ldots$. . . . . . . . 87

3.19 Classificações incorretas produzidas pelo nosso classificador 5-NN, usando o banco de imagens MNIST [52]. Como feito em [9], no topo de cada dígito manuscrito, exibimos seu identificador, seguido pela correta classificação e pelo resultado incorreto produzido pelo nosso algoritmo. . . . . . . . . . . 88

3.20 Classificações incorretas produzidas pelo nosso classificador 5-NN, usando o banco de imagens MNIST [52]. Como feito em [9], no topo de cada dígito manuscrito, exibimos seu identificador, seguido pela correta classificação e pelo resultado incorreto produzido pelo nosso algoritmo. . . . . . . . . . . 89

3.21 Classificações incorretas produzidas pelo nosso classificador 5-NN, usando o banco de imagens MNIST [52]. Como feito em [9], no topo de cada dígito manuscrito, exibimos seu identificador, seguido pela correta classificação e pelo resultado incorreto produzido pelo nosso algoritmo. . . . . . . . . . . 90

3.22 Resumo da colorização assistida por computador. . . . . . . . . . . . . . . 91

3.23 Arestas são criadas entre regiões adjacentes. . . . . . . . . . . . . . . . 93 
3.24 Exemplo 'face'. Partindo do quadro colorido, representado pelo grafo modelo. O objetivo é colorir o próximo quadro (não-colorido), representado pelo grafo de entrada. Os resultados, do nosso método e do método de Bezerra et al. [14], são exibidos para comparação. . . . . . . . . . . . . . 95

3.25 Exemplos de colorização das animações (a) 'cufa', (b) 'lobinho' e (c) 'calango'. Para cada exemplo, apresentamos o modelo e a entrada colorizada pelo nosso método, respectivamente. . . . . . . . . . . . . . 96

3.26 Exemplos de colorização da animação 'cufa'. Quadros 35 a $40 . \quad$. . . . . . 98

3.27 Exemplos de colorização da animação 'cufa'. Quadros 40 a 44 . . . . . . . 99

3.28 Exemplos de colorização da animação 'calango'. Quadros 21 a 25. . . . . . 100

3.29 Exemplos de colorização da animação 'lobinho'. Quadros 25 a 29. . . . . . 101

3.30 Exemplos de colorização da animação 'lobinho'. Quadros 29 a 32. . . . . . 102

4.1 (a) Imagem de entrada. (b) Traços do usuário sobrepostos na imagem.

(c) Segmentação. . . . . . . . . . . . . . . . . . . . . . . . . . . 109 


\section{Lista de Tabelas}

2.1 Comparação entre as abordagens DG, BGM, BGM+TPS e GA, usando os pares originais de [2]. Para cada método, são exibidos o parâmetro utilizado e a correspondente taxa de erro. . . . . . . . . . . . . . 29

2.2 Tempos de execução para a computação da segmentação inicial pelo algoritmo de casamento, e para o refinamento pelo pós-processamento, usando

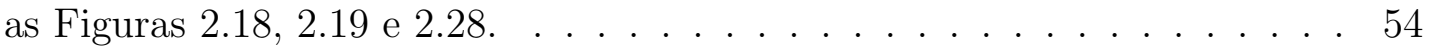

2.3 Regiões do watershed: quantidade original e reduzida, respectivamente, usando as Figuras 2.18, 2.19 e $2.28 . \quad \ldots \ldots \ldots \ldots \ldots \ldots$

2.4 Comparação quantitativa, usando as imagens de Blake e associados [16]. . 55

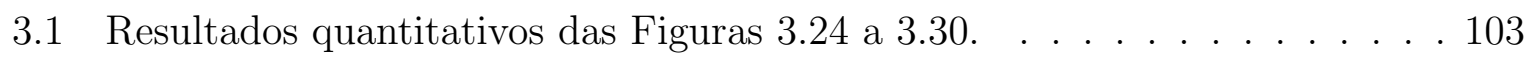

4.1 Resumo do desempenho das duas contribuições, DG e BP, sobre as quatro aplicações tratadas neste trabalho. . . . . . . . . . . . . . 105 


\section{Capítulo 1}

\section{Introdução}

\subsection{Motivação}

Casamento de padrões de pontos é um problema fundamental em reconhecimento de padrões. O objetivo é encontrar uma correspondência entre dois conjuntos de pontos, associados a características relevantes de objetos ou entidades, mapeando os pontos de um conjunto no outro, conforme ilustrado na Figura 1.1. Para encontrar um mapeamento, é desejável codificar os objetos por representações eficientes, de maneira que apenas as características relevantes sejam consideradas na comparação entre pares de objetos.

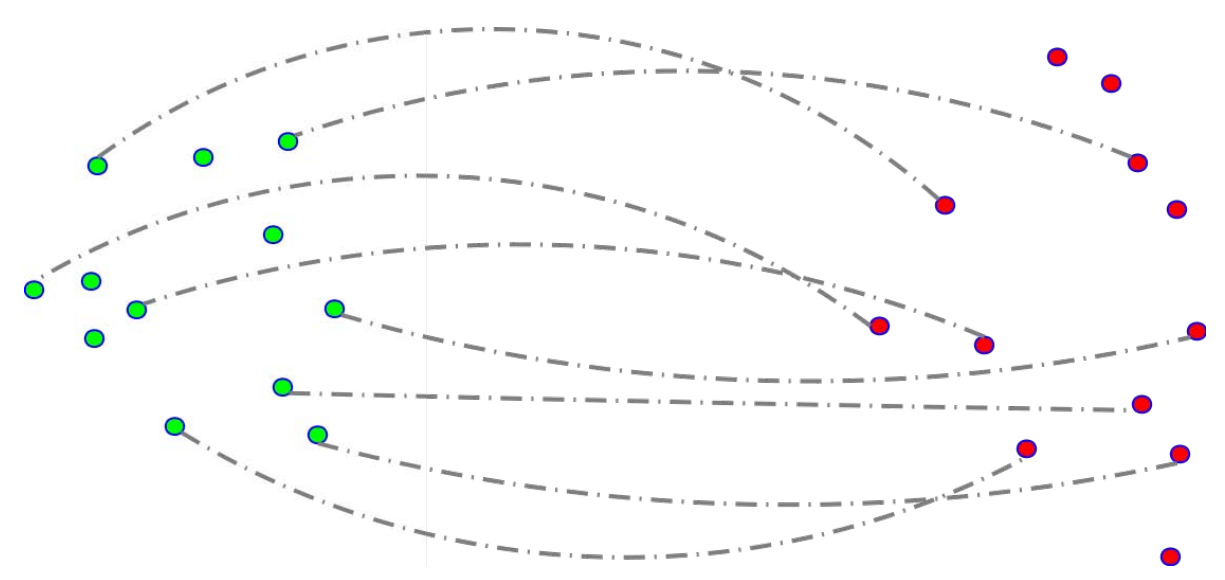

Figura 1.1: Dois padrões de pontos. Os pontos correspondentes estão conectados por linhas pontilhadas. 
Este problema possui uma variedade de aplicações. Em visão computacional, o reconhecimento de objetos pode ser realizado através da comparação de objetos representados por padrões de pontos, resumindo as características locais, em termos de aparência, e as relações espaciais entre os pontos [9]. Um outro exemplo é o problema envolvendo imagens estéreo, cujo objetivo é encontrar uma correspondência entre os pontos de um par de imagens, uma ligeiramente deslocada em relação a outra, adquiridas por um sistema binocular de câmeras [18]. Em processamento de imagens, existe o problema de registro, onde através de uma correspondência entre pontos, é possível computar uma transformação de modo a alinhar a imagem de entrada com a imagem de referência [30]. Existem muitas outras aplicações envolvendo algum tipo de casamento, por exemplo, na área de proteômica, onde análises diferenciais são extremamente importantes para diagnóticos médicos e desenvolvimento de medicamentos [74]. Outros exemplos incluem biometria, mineração de dados, entre outros [32].

\subsection{Objetivos}

O foco principal deste trabalho é sobre as áreas de visão computacional, processamento e análise de imagens, com o objetivo de desenvolver métodos eficientes para problemas práticos envolvendo casamento entre pontos provenientes de pares de imagens, explorando os requisitos específicos de cada aplicação.

Conforme descrito em [21], o problema de casamento de padrões de pontos tem sido tipicamente tratado como um problema de casamento entre grafos desde a década de 1970, quando estudos pioneiros foram publicados nesta área, por exemplo [8, 40]. No casamento entre grafos, os padrões de pontos são codificados por grafos, onde pontos são representados por vértices e arestas correspondem à estrutura, modelando as configurações espaciais da distribuição dos pontos.

Para codificar as informações relevantes de objetos, vértices e arestas podem ser atribuídos com vetores de características. Uma vez que os padrões são codificados por grafos, o problema de casamento de padrões de pontos se transforma em um problema de casamento entre grafos, cujo objetivo é encontrar um mapeamento de um conjunto de vértices de um grafo para um conjunto de vértices do outro, de modo a minimizar uma função custo, representando dissimilidades globais entre os dois objetos, avaliando 
os atributos associados aos dois grafos.

Desde o entusiasmo inicial do fim da década de 1970, inúmeras contribuições têm sido desenvolvidas para obter algoritmos cada vez mais eficientes para o problema de casamento entre grafos. Após um período de menor interesse a partir da década de 1980 até o começo da década de 1990, um crescente esforço têm sido realizado junto à comunidade científica, motivado pela modernização dos computadores [32]. Para grafos arbitrários, o problema de casamento entre grafos é NP-completo, incentivando pesquisadores a desenvolver aproximações eficientes com complexidade de tempo polinomial.

\subsection{Contribuições}

Uma análise da extensa literatura indica que a maioria dos métodos atuais consiste de técnicas sofisticadas para o problema genérico de casamento entre grafos, incluindo algoritmos de busca, técnicas de otimização contínua e métodos espectrais.

Ao contrário dos métodos sofisticados voltados para o problema genérico, propomos duas técnicas eficientes para o problema de casamento entre grafos, explorando as relações espaciais dentre os requisitos específicos de cada aplicação para facilitar o processo de casamento. O primeiro algoritmo é baseado em grafos auxiliares, chamados de grafos deformados (DGs), usados para evitar a comparação direta entre os dois grafos sendo casados. O segundo método explora as relações espaciais através de um novo termo de Markov na função custo, minimizada por um eficiente algoritmo de propagação de crenças (BP) [39]. Experimentos usando dados públicos são exibidos para permitir comparações. Os dois métodos são aplicados aos seguintes problemas práticos:

- casamento entre gels de eletroforese 2D [65, 66] (em colaboração com Alvaro Pardo, Universidade Católica do Uruguai),

- segmentação interativa de imagens naturais [1] (em colaboração com Luís Augusto Consularo, Tribunal Superior Eleitoral; Ana Beatriz Vicentim Graciano, Instituto de Matemática e Estatística; e Isabelle Bloch, TELECOM ParisTech),

- casamento entre formas ou shape matching [63], 
- e colorização assistida por computador [67] (em colaboração com Luiz Velho, Instituto Nacional de Matemática Pura e Aplicada).

A limitação principal do primeiro método, baseado em DGs, é que ele considera apenas um dos dois conjuntos de arestas para o cálculo do casamento. As arestas consideradas são representadas em um modelo, sendo este método mais adequado para problemas interativos, que aproveitam o conhecimento a priori fornecido pelo usuário, por exemplo, para centralizar o modelo sobre o conjunto de pontos da entrada, a serem classificados.

O segundo método, baseado em BP, não sofre esta restrição, considerando os dois conjuntos de arestas relativos aos dois grafos sendo casados. Porém, a qualidade da técnica baseada em BP é bastante conhecida apenas para grafos específicos, como por exemplo árvores e grafos com um único loop [94, 95], sendo a segunda abordagem adequada para problemas que exigem 'poucas' arestas nos dois grafos sendo casados.

\subsection{Organização}

Com o objetivo de descrever os dois métodos propostos como contribuições originais deste trabalho, esta Tese está organizada da seguinte maneira. A primeira abordagem, baseada em DGs, é detalhada no Capítulo 2, enquanto o segundo algoritmo, baseado em BP, é descrito no Capítulo 3. Cada um destes capítulos é acompanhado por teoria e aplicações, incluindo uma revisão bibliográfica sobre o problema concreto sendo tratado, juntamente com experimentos computacionais. Finalmente, conclusões e trabalhos futuros são desenhados no Capítulo 4, onde é dado um resumo dos resultados experimentais envolvendo as duas contribuições sobre as quatro aplicações tratadas neste trabalho, com o objetivo de fornecer mais informações para guiar o leitor sobre qual das duas abordagens seria mais interessante para uma determinada aplicação. 


\section{Capítulo 2}

\section{Casamento de padrões de pontos via casamento entre grafos}

Antes de descrever nossa primeira contribuição, o casamento de padrões de pontos é formulado como um problema de casamento entre grafos, acompanhado por um resumo sobre sua extensa literatura.

A primeira abordagem é baseada em grafos auxiliares, chamados de grafos deformados (DG), usados para uma avaliação eficiente das informações estruturais codificadas pelas arestas de um dos dois grafos sendo casados. O algoritmo resultante é aplicado no casamento entre gels de eletroforese bidimensional (2DEM) [65, 66] e no problema de segmentação interativa de imagens naturais (INIS) [1]. Para o 2DEM, além de utilizar uma abordagem gulosa para a otimização, este método combina a técnica iterative closest point (ICP) [13] para obter invariância quanto a translação.

\subsection{Casamento entre grafos}

Cada aplicação é tratada como um problema de casamento de padrões de pontos, associados a características relevantes de objetos ou entidades. O objetivo é encontrar uma correspondência entre os pontos dos dois conjuntos.

Neste trabalho, para o cálculo da correspondência, os objetos são codificados por grafos, representando poderosas ferramentas para codificar padrões do mundo real, resu- 
Casamento de padrões de pontos via casamento entre grafos

mindo as características relevantes de aparência ou características locais, e a estrutura ou relações entre as características locais. Estas informações de aparência e de estrutura são consideradas na comparação entre pares de objetos. Neste caso, cada ponto é representado por um vértice, e uma aresta é criada entre dois vértices para representar uma relação entre as extremidades da aresta. O objetivo do casamento entre grafos é encontrar um mapeamento entre os vértices dos dois grafos, representando os dois objetos sendo comparados.

\subsubsection{Exato vs Inexato}

O casamento entre grafos pode ser exato ou inexato. Um casamento exato é caracterizado

pela restrição de preservação de arestas no seguinte sentido: se dois vértices são conectados por uma aresta no primeiro grafo, então os seus correspondentes também devem estar conectados por uma aresta no segundo grafo [32]. Entretanto, em muitos problemas práticos, esta restrição é muito forte devido ao fato de que os grafos estão sujeitos a deformações resultantes de ruídos inseridos no processo de aquisição dos atributos e pela variabilidade intrínseca dos padrões. No lugar de proibir, o casamento inexato penaliza as correspondências que não obedecem a restrição de preservação de arestas, incrementando o valor da função custo.

Conforme descrito em [32], o caso inexato pode ser visto como uma generalização dos algoritmos de casamento exato: "Algoritmos ótimos para o casamento inexato sempre encontram uma solução representando o mínimo global da função custo. Se existir uma solução para o seu correspondente caso exato, ela também será encontrada por tais algoritmos." Esta Tese trabalha com casamentos inexatos e usamos o termo casamento no sentido inexato.

\subsubsection{Homomorfismo vs Isomorfismo}

A função de mapeamento pode representar, basicamente, um homomorfismo (injetora, muitos-para-um) ou um isomorfismo (bijetora, um-para-um). Na prática, o isomorfismo é muito restritivo e uma versão mais relaxada de casamento é o isomorfismo de subgrafo, que requer um isomorfismo entre um dos dois grafos e um subgrafo induzido por vértices do outro grafo. Um caso ainda mais relaxado de isomorfismo é o subgrafo comum máximo 
(MCS), que mapeia um subgrafo do primeiro grafo para um subgrafo isomórfico do segundo grafo. Todos os problemas de casamento descritos acima são NP-completos, exceto pelo isomorfismo entre grafos, para o qual ainda não foi demonstrado se pertence ou não a NP [32].

Para o casamento entre gels de eletroforese 2D, o objetivo é encontrar um MCS (correspondência um-para-um), enquanto para o problema de segmentação interativa de imagens naturais, desejamos encontrar um mapeamento representando um homomorfismo (muitos-para-um).

\subsubsection{Histórico}

O problema genérico de casamento entre grafos possui uma longa história, com origens na década de 1970, por exemplo, nos trabalhos pioneiros de Barrow e Popplestone [8] e de Fischler e Elschlager [40], que demonstraram a importância do uso das relações estruturais no contexto de reconhecimento de objetos em imagens.

O problema do casamento entre grafos é conhecido por ser custoso no caso geral, com sofisticados métodos, cujas complexidades computacionais variam de $O\left(n^{6}\right)$ até $O\left(n^{2}\right)$. Nesta seção, descrevemos brevemente três principais classes de algoritmos de casamento. Nosso objetivo não é detalhar com profundidade, mas sim ilustrar as principais tendências de pesquisa atuais, incluindo algoritmos baseados em busca, técnicas de otimização contínua e métodos espectrais. Para uma profunda revisão dos métodos, veja [32] .

\section{Algoritmos baseados em busca}

Tsai e Fu [88] foram os pioneiros na técnica baseada em buscas por pares de vértices correspondentes. A idéia principal é construir incrementalmente um mapeamento, expandindo iterativamente a solução inicialmente vazia através da adição de novos pares. A busca é guiada pelo custo do mapeamento parcial e por uma estimação de custo dos futuros pares, envolvendo os vértices ainda não-classificados. Os autores de [88] defini-

ram os grafos relacionais com atributos (ARGs), propondo custos baseados em operações de edição de grafos, explorando substituição de vértices e de arestas, e restringindo a aplicação do método para grafos isomórficos. Mais tarde, foi estendido para incluir outras 
Casamento de padrões de pontos via casamento entre grafos

operações como inserção e remoção [89], generalizando sua aplicação para isomorfismos de subgrafos.

Seguindo mais adiante com estas idéias, Sanfeliu e Fu [77] definiram uma distância baseada nas operações de edição de grafos, considerando um conjunto canônico de operações, como substituições de vértices e de arestas, e divisão e fusão de vértices. Eshera e Fu [37] propuseram um método para o cálculo da distância, baseado em definições apropriadas de subgrafos simples para obter casamentos ótimos, usados como aproximações para o problema original, via programação dinâmica.

Na mesma linha incremental de construção de soluções, Shapiro e Haralick [80] propuseram um algoritmo branch and bound para o cálculo de homomorfismos. Mais tarde, os mesmos autores propuseram uma métrica para avaliar descrições relacionais [81].

Outros métodos baseados em heurísticas para estimar os custos dos futuros pares foram propostos na literatura, por exemplo [11].

\section{Otimização contínua}

No lugar de aplicar buscas discretas, uma alternativa é tratar o problema de casamento como um problema de otimização contínua, permitindo o uso de técnicas bastante úteis, que apesar de não garantirem uma solução ótima global, podem proporcionar soluções rápidas e precisas.

Os métodos mais importantes desta classe são baseados em relaxação probabilística (probabilistic relaxation labeling). Fischler e Elschlager [40] foram os pioneiros nesta técnica. Mais tarde, Rosenfeld et al. [75] introduziram uma formalização para a técnica de relaxação, através de uma heurística iterativa usada para atualizar as verosimilhanças das correspondências, de acordo com as evidências providas pelos vértices vizinhos.

Kittler e Hancock [49] e Christmas et al. [28] propuseram formulações probabilísticas mais sólidas, resultando em um arcabouço probabilístico Bayesiano de relaxação que provou ser bastante útil para problemas de casamento. Hancock e associados [47, 57, 87] exploraram várias abordagens probabilísticas, introduzindo técnicas bastante sólidas de casamento, de grande impacto sobre a comunidade científica.

Gold e Rangarajan [44] propuseram o algoritmo graduated assignment, aplicando a 
técnica graduated nonconvexity para evitar mínimos locais. Este algoritmo apresentou resultados expressivos quando comparado ao padrão dos algoritmos de relaxação descritos anteriormente, sendo um dos algoritmos usados para avaliar a qualidade das duas contribuições propostas neste trabalho.

Recentemente, algoritmos de relaxação baseados em campos aleatórios de Markov (MRF) têm sido propostos na literatura, por exemplo [20], permitindo o uso do poderoso arcabouço MAP-MRF (veja Section 3.2), com poderosas técnicas de otimização, incluindo programação semi-definida [78, 85] e propagação de crenças (BP) [36]. Nossa segunda contribuição segue esta tendência, baseada em um algoritmo eficiente baseado em BP [39] (veja Capítulo 3).

\section{Métodos espectrais}

Um interesse especial por parte dos pesquisadores têm recaído sobre análises espectrais para mensurar similaridades entre grafos. A idéia básica é que os autovalores e autovetores da matriz de adjacência de um grafo são invariantes com relação a permutações dos vértices, resultando em um mesmo conjunto de autovalores e autovetores para grafos isomórficos. Apesar do fato que a volta nem sempre é válida, as propriedades espectrais e a complexidade computacional para a decomposição espectral são bastante atrativas.

Um dos primeiros trabalhos nesta área foi devido a Umeyama [90]. Por decomposição espectral, o autor derivou uma expressão simples para otimizar a função custo, assumindo que os grafos são isomórficos.

Carcassoni e Hancock [24] desenvolveram um método baseado em agrupamento dos prováveis vértices correspondentes, explorando a hierarquia resultante para mapear primeiramente os grupos e depois os vértices dentro dos grupos. Diferentemente do método proposto por Umeyama, esta técnica pode ser aplicada a grafos com tamanhos diferentes.

Abordagens misturando técnicas de relaxação e métodos espectrais têm sido propostas recentemente. Por exemplo, podemos citar os algoritmos spectral matching [53] e spectral matching with affine constraints [33]. A diferença principal entre os dois é que, enquanto o primeiro aplica as restrições de mapeamento (por exemplo, um-para-um) em um passo separado (discretization step), o segundo impõe as restrições de mapeamento como parte integrante da otimização, durante a análise espectral. 


\subsection{Formulação via atribuição quadrática}

Com o objetivo de mensurar a qualidade de um mapeamento, muitas aplicações envolvendo casamento podem ser formuladas através de uma atribuição quadrática (quadratic assignment problem), onde o termo linear avalia as característica locais de aparência, enquanto o termo quadrático da função custo avalia as informações estruturais. O problema de atribuição quadrática é NP-difícil, incentivando pesquisadores a desenvolverem técnicas eficientes de aproximação.

Neste trabalho, usamos grafos relacionais com atributos (ARGs) [88] para codificar os dois tipos de informações, aparência e estrutura. Basicamente, um ARG é um grafo orientado, onde as informações de aparência são representadas por atributos de vértices, e as informações estruturais como atributos de arestas.

Dados dois ARGs, um de entrada e outro representando o modelo, nos concentramos no problema de casamento entre grafos atribuídos, onde desejamos encontrar um mapeamento entre vértices da entrada e vértices do modelo, avaliando os atributos dos vértices e das arestas dos dois grafos através de uma função custo. Denotamos o grafo modelo por $G_{m}=\left(V_{m}, E_{m}, \mu, \nu\right)$, um vértice do modelo por $v_{m} \in V_{m}$, seu atributo por $\mu\left(v_{m}\right)$, uma aresta (orientada) do modelo por $e_{m} \in E_{m}$ e seu atributo por $\nu\left(e_{m}\right)$. Notações similares são usadas para o grafo de entrada $G_{i}$. Neste texto, ARGs são simplesmente chamados de grafos e os dois termos são usados como sinônimos. Dados um grafo de entrada e um grafo modelo, representando os dois objetos ou padrões a serem casados, o objetivo é encontrar um mapeamento $f: V_{i} \rightarrow V_{m}$, de vértices da entrada para vértices do modelo, minimizando a seguinte função custo ou função energia:

$$
E(f)=\lambda_{1} \sum_{v_{i} \in V_{i}} d_{A}\left(v_{i}, f\left(v_{i}\right)\right)+\left(1-\lambda_{1}\right) \sum_{\left(v_{i}, v_{i}^{\prime}\right) \in E_{i}} d_{S}^{\prime}\left(\left(v_{i}, v_{i}^{\prime}\right),\left(f\left(v_{i}\right), f\left(v_{i}^{\prime}\right)\right)\right) .
$$

Ambos termos, linear e quadrático, são balanceados por um parâmetro $\lambda_{1}$, um número real entre 0 e 1. 


\subsubsection{Termo linear: distância de aparência $\left(d_{A}\right)$}

O termo linear $d_{A}$ compara pares de vértices $\left(v_{i}, v_{m}\right)$ representando os pontos correspondentes, avaliando diretamente os atributos $\mu\left(v_{i}\right)$ e $\mu\left(v_{m}\right)$. Para cada aplicação específica, usamos diferentes atributos para os vértices. Por exemplo, para o casamento entre gels de eletroforese 2D, os atributos representam informações de shape context [9], enquanto para o problema de segmentação interativa de imagens naturais, os atributos representam informações de cores.

\subsubsection{Termo quadrático: distância estrutural $\left(d_{S}\right)$}

O termo quadrático $d_{S}$ representa a distância estrutural, usada para avaliar atributos de arestas, considerando penalidades geométricas usadas para caracterizar as relações espaciais, penalizando mudanças na orientação e na distância entre os pontos.

Para todas as aplicações tratadas nesta Tese, adotamos as seguintes informações estruturais. A idéia principal é que cada aresta orientada possui um vetor correspondente como seu atributo, onde cada vetor é definido pelas coordenadas dos pontos representados pelas extremidades da aresta. Inspirado no trabalho descrito em [26], as posições relativas são avaliadas pela seguinte equação, que compara dois vetores, $\overrightarrow{v_{1}}$ e $\overrightarrow{v_{2}}$, avaliando o ângulo formado e a diferença de comprimento entre os dois vetores:

$$
c_{\mathrm{vec}}\left(\overrightarrow{v_{1}}, \overrightarrow{v_{2}}\right)=\lambda_{2} \frac{|\cos \theta-1|}{2}+\left(1-\lambda_{2}\right) \frac{|| \overrightarrow{v_{1}}|-| \overrightarrow{v_{2}}||}{C_{S}}
$$

onde $\theta$ representa o ângulo entre os dois vetores e $|\vec{v}|$ denota o módulo ou comprimento de um vetor. $C_{S}$ é um termo de normalização, representando a diferença máxima de tamanho entre dois vetores.

O primeiro termo da Equação 2.2 representa o custo 'angular', atribuindo custos elevados a vetores opostos. O segundo termo representa o custo 'modular', atribuindo valor proporcional à diferença entre os comprimentos dos dois vetores, normalizado por uma constante $C_{S}$ para manter os valores entre 0 e 1 . O parâmetro $\lambda_{2}$ varia entre 0 e 1 , para balancear a importância entre os dois termos, angular e modular. Penalidades geométricas similares à equação apresentada acima também foram exploradas em $[10,53]$. A definição completa da distância estrutural $d_{S}$, para o algoritmo baseado em DG, é feita na Seção 2.3.2. 


\subsubsection{Desafio em casamento entre grafos}

O desafio principal do problema de casamento entre grafos consiste em desenvolver maneiras eficientes para casar dois grafos com tamanhos diferentes, por exemplo, com quantidades distintas de vértices. Neste caso, não é trivial avaliar os atributos das arestas de maneira eficiente. Resultados experimentais exibidos na literatura mostram que grande parte dos algoritmos disponíveis possuem uma tendência de produzir resultados piores quando submetidos a grafos com tamanhos distintos. Esta deterioração se mostra mais evidente quando a diferença de tamanho entre os grafos aumenta, conforme observado em [23].

Em situações reais, outliers podem surgir devido a diferenças entre os padrões ou devido a erros de detecção. Neste caso, os grafos de entrada e o de modelo tendem a possuir quantidades distintas de vértices, dificultando a avaliação estrutural entre os dois, especialmente para grandes instâncias do problema.

Para avaliar de maneira eficiente as informações codificadas nas arestas, exploramos as relações espaciais entre os pontos. Nossa primeira abordagem compara atributos de arestas usando grafos auxiliares, chamados de grafos deformados, onde a idéia principal é sempre efetuar a comparação estrutural entre dois grafos de mesma topologia.

\subsection{Casamento via grafos deformados}

\subsubsection{Grafos deformados (DGs)}

Para uma avaliação eficiente das informações estruturais codificadas pelos atributos de arestas, grafos auxiliares, chamados de grafos deformados (DGs), são usados em nossa primeira abordagem. Dado um par $\left(v_{i}, v_{m}\right), v_{i} \in V_{i}, v_{m} \in V_{m}$, de modo a avaliar o impacto estrutural causado ao associar um vértice de entrada $v_{i}$ a um vértice modelo $v_{m}$, primeiramente computamos o DG correspondente, $G_{d}\left(v_{i}, v_{m}\right)$, representando uma deformação local no grafo modelo $G_{m}$ com respeito aos atributos de arestas causada pela simulação de troca de coordenadas do vértice $v_{m}$. $G_{d}\left(v_{i}, v_{m}\right)$ é calculado da seguinte maneira. Este grafo auxiliar é quase uma cópia do modelo $G_{m}$, com os mesmos vértices e arestas, e mesmos atributos de arestas, exceto pelos atributos correspondentes às arestas deformadas, 
resultantes da troca de coordenadas do vértice $v_{m}$ pelas coordenadas do vértice $v_{i}$. Esta troca de coordenadas é feita na cópia correspondente de $v_{m}$ em $G_{d}\left(v_{i}, v_{m}\right)$, resultando no vértice deformado $v_{d}$. As arestas deformadas são aquelas que possuem uma extremidade em $v_{d}$, conforme ilustradas na Figura 2.1(c).

Note que apenas os atributos das arestas deformadas em $G_{d}\left(v_{i}, v_{m}\right)$ podem variar em relação aos seus correspondentes no modelo $G_{m}$. Logo, durante a comparação entre os dois grafos, $G_{d}\left(v_{i}, v_{m}\right)$ e $G_{m}$, apenas as arestas deformadas são examinadas, permitindo uma eficiente avaliação estrutural.

\subsubsection{Distância estrutural $\left(d_{S}\right)$}

Para as duas aplicações, casamento entre gels de eletroforese 2D e segmentação interativa de imagens naturais, as informações estruturais extraídas do modelo foram utilizadas para o cálculo dos casamentos, conforme ilustrado à esquerda da Figura 2.1(b). O objetivo dos DGs é avaliar as deformações não-rígidas entre os pontos correspondentes. Para efetuar esta tarefa de maneira eficiente, as informações de adjacência presentes em $E_{i}$ são ignoradas para evitar possíveis incompatibilidades topológicas entre os grafos de entrada e de modelo. Para um dado par $\left(v_{i}, v_{m}\right)$, primeiramente calculamos o seu correspondente DG, $G_{d}\left(v_{i}, v_{m}\right)$, possibilitando uma eficiente avaliação estrutural dada pela seguinte expressão, no papel do termo quadrático da Equação 2.1:

$$
d_{S}\left(G_{d}\left(v_{i}, v_{m}\right), G_{m}\right)=\frac{1}{\left|E\left(v_{d}\right)\right|} \sum_{e_{d} \in E\left(v_{d}\right)} c_{\mathrm{vec}}\left(\nu\left(e_{d}\right), \nu\left(e_{m}\right)\right)
$$

onde $c_{\mathrm{vec}}(\cdot)$ corresponde a função de penalidade geométrica definida pela Equação 2.2, $E\left(v_{d}\right)$ denota o conjunto de arestas deformadas com uma extremidade em $v_{d},\left|E\left(v_{d}\right)\right|$ denota o seu tamanho, $e_{m}$ é a aresta do modelo correspondente a aresta deformada $e_{d}$, e $\nu\left(e_{d}\right)$ e $\nu\left(e_{m}\right)$ são os vetores correspondentes aos atributos das arestas. A Equação 2.3 representa o custo médio entre as arestas deformadas e suas respectivas arestas do modelo.

$\mathrm{Na}$ avaliação estrutural usando DGs, cada $v_{i} \in V_{i}$ tende a ser associado ao vértice 'mais próximo' $v_{m} \in V_{m}$, conforme ilustrado à direita da Figura 2.1(b). Na Figura 2.1(c), é ilustrado um exemplo de grande deformação devido a um vértice $v_{i}$ distante de $v_{m}$. 


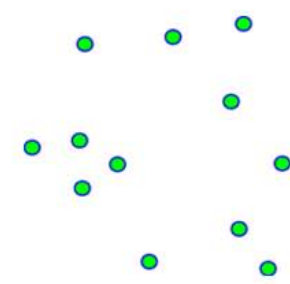

(a)
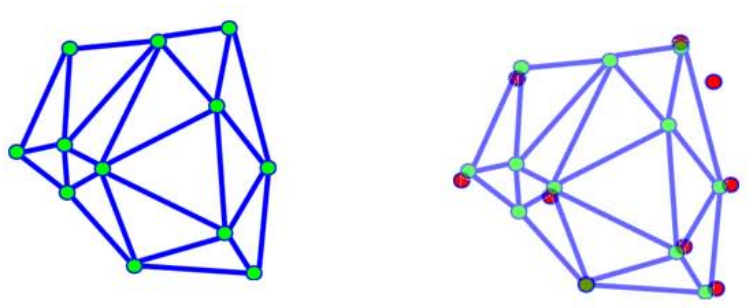

(b)
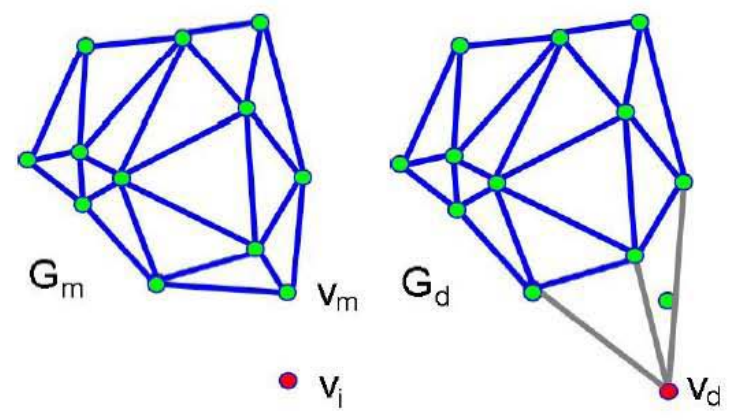

(c)

Figura 2.1: (a) Dois padrões de pontos, usados como modelo e entrada, respectivamente. (b) Informação estrutural extraída do modelo, à esquerda, e sobreposto na entrada, à direita. (c) Grafo modelo $G_{m}$ e grafo deformado $G_{d}$ devido ao par $\left(v_{i}, v_{m}\right)$, respectivamente. 


\subsubsection{Otimização}

Usando DGs, a avaliação dos atributos de arestas dependem apenas do par $\left(v_{i}, v_{m}\right)$ correntemente examinado. Logo, cada par pode ser examinado de maneira independente pela seguinte expressão:

$$
E\left(v_{i}, v_{m}\right)=\lambda_{1} d_{A}\left(v_{i}, v_{m}\right)+\left(1-\lambda_{1}\right) d_{S}\left(G_{d}\left(v_{i}, v_{m}\right), G_{m}\right)
$$

e a Equação 2.1 pode ser reescrita da seguinte maneira:

$$
E(P)=\sum_{\left(v_{i}, v_{m}\right) \in P} E\left(v_{i}, v_{m}\right)
$$

onde $P$ é o conjunto dos pares representando um homomorfismo entre vértices da entrada e vértices do modelo. Para minimizar a Equação 2.5, usamos o seguinte algoritmo guloso.

\section{Calculahomomorfismo $\left(G_{i}, G_{m}\right)$}

$1 P \leftarrow \emptyset$

2 para cada vértice $v_{i} \in V_{i}$

3 faça

$4 \quad c_{\min } \leftarrow \infty ; v_{\min } \leftarrow \mathrm{NULL}$

$5 \quad$ para cada vértice $v_{m} \in V_{m}$

6 faça

$7 \leftarrow E\left(v_{i}, v_{m}\right)$

$8 \quad$ se $c<c_{\min }$

9 faça

10

11

$$
c_{\min } \leftarrow c ; v_{\min } \leftarrow v_{m}
$$

\section{2 devolva $P$}

O próximo algoritmo recebe os dois grafos, o de entrada e o modelo, e devolve um conjunto $P^{\prime}$ de pares $\left(v_{i}, v_{m}\right)$ representando um MCS entre $G_{i}$ e $G_{m}$. O objetivo é descartar possíveis outliers, ou pontos sem correspondência, da solução inicial $P$ durante o pósprocessamento seguinte. Para cada $v_{m} \in V_{m}$, o algoritmo avalia o custo de cada par $\left(v_{i}, v_{m}\right) \in P, v_{i} \in V_{i}$, através da Equação 2.4, mantendo apenas um único par $\left(v_{i}^{\prime}, v_{m}\right)$ em $P^{\prime}$ associado ao menor custo, descartando os demais pares $\left(v_{i}, v_{m}\right), v_{i} \neq v_{i}^{\prime}$, de $P^{\prime}$. Os pares descartados são considerados como outliers, sem correspondência. 
CalculamCS $\left(G_{i}, G_{m}\right)$

$1 \quad P \leftarrow$ CalculaHomomorfismo $\left(G_{i}, G_{m}\right)$

2 Pós-processamento de $P$ :

para cada $v_{m}$, mantenha apenas um único par $\left(v_{i}, v_{m}\right)$, de custo mais baixo, em $P^{\prime}$.

3 devolva $P^{\prime}$

Por um lado, observamos que para obter 'bons' casamentos entre padrões complexos de pontos, com grandes deformações não-rígidas entre os pontos correspondentes, é preciso efetuar alinhamentos adequados entre o grafo modelo e o conjunto de pontos da entrada para uma avaliação estrutural via DGs. Por outro lado, se as correspondências são conhecidas, o alinhamento pode ser facilmente estimado, resultando em um problema similar ao do 'ovo e da galinha'. Neste trabalho, exploramos a estratégia ICP [13], que alterna dois passos, estimação das correspondências e cálculo do alinhamento, que são repetidos até a convergência do algoritmo ou até que ele atinja um número máximo de iterações. Esta técnica é resumida no algoritmo a seguir:

\section{CalculamCSICP $\left(G_{i}, G_{m}\right)$}

1 Inicialize os deslocamentos $d_{x}^{0}$ e $d_{y}^{0}$

2 repita

$3 \quad$ Atualize as coordenadas em $G_{m}$ usando $d_{x}^{t-1}$ e $d_{y}^{t-1}$.

$4 \quad P^{t} \leftarrow \operatorname{CalculamCS}\left(G_{i}, G_{m}\right)$.

$5 \quad$ Estime os deslocamentos $d_{x}^{t}$ e $d_{y}^{t}$ a partir de $P^{t}$.

6 até o resultado convergir ou o algoritmo atingir um \# máximo de iterações

7 devolva $P^{t}$

Para alinhar o grafo modelo sobre o padrão de pontos da entrada de maneira apropriada, deve-se estimar um deslocamento apropriado, representado pelo par $\left(d_{x}, d_{y}\right)$, usado para atualizar as coordenadas dos vértices do modelo, adicionando $\left(d_{x}, d_{y}\right)$ a cada ponto do modelo. Note que não há necessidade de recálculo de SC, mantendo assim a eficiência do método proposto.

Conforme descrito em [13], o método ICP apresenta propriedades de convergência bastante desejáveis. Entretanto, esta técnica garante a convergência somente para mínimos locais. Desta forma, a qualidade dos resultados dependem de uma inicialização adequada dos parâmetros. Por exemplo, para o problema 2DEM, o deslocamento inicial 
foi inicializado de maneira automática, usando-se as coordenadas correspondentes às medianas de cada padrão de pontos, atribuindo $d_{x}^{0}=\operatorname{median}\left\{x_{i}\right\}-\operatorname{median}\left\{x_{m}\right\}$ e $d_{y}^{0}=$ median $\left\{y_{i}\right\}-\operatorname{median}\left\{y_{m}\right\}$, onde median $\left\{x_{i}\right\}$ representa a mediana no eixo $X$ dentre todos os pontos da entrada. Similarmente median $\left\{y_{i}\right\}$ representa a mediana no eixo $Y$. Median $\left\{x_{m}\right\}$ e median $\left\{y_{m}\right\}$ são definidos de maneira análoga. Ainda para o problema 2DEM, mostramos que, para pares complexos de padrões de pontos, podemos obter resultados de qualidade, explorando o conhecimento a priori do usuário na inicialização dos deslocamentos $\left(d_{x}^{0}, d_{y}^{0}\right)$ (veja Seção 2.4.4, experimento 2).

Em uma dada iteração $t$, para calcular os deslocamentos $d_{x}^{t}$ e $d_{y}^{t}$ a partir das correspondências estimadas, consideramos a mediana de cada eixo, $X$ e $Y$, dos deslocamentos ocorridos entre os pontos correspondentes em $P^{t}$, atribuindo $d_{x}^{t}=\operatorname{median}\left\{x_{i}-x_{m}\right\}$, onde $x_{i}$ and $x_{m}$ são as coordenadas no eixo $X$ dos vértices correspondentes, da entrada e do modelo. Analogamente, $d_{y}^{t}=\operatorname{median}\left\{y_{i}-y_{m}\right\}$.

\subsection{Aplicação 1: casamento entre gels de eletroforese $2 \mathrm{D}$}

Eletroforese bidimensional $(2 \mathrm{DE})^{1}$ é um método bastante conhecido para separação de proteínas, extremamente utilizado na área de proteômica [74]. A idéia principal é separar as proteínas contidas em uma amostra usando duas propriedades independentes, como por exemplo, ponto isoelétrico e massa. Um exemplo de par de imagens obtidas por esta técnica é ilustrado na Figura 2.2, juntamente com a correspondência entre os pontos detectados nas duas imagens.

Cada mancha na imagem representa um acúmulo de proteína, cujo tamanho depende da quantidade de proteína presente na amostra. Uma escala de cinza é posicionada no topo de cada imagem para possibilitar uma calibração dos níveis de cinza. Aparentemente simples, o processamento manual de imagens 2DE é bastante trabalhoso. Durante a comparação das amostras, um único experimento pode envolver um grande número de

\footnotetext{
${ }^{1}$ Esta parte foi desenvolvida com o professor Alvaro Pardo, que nos proporcionou uma importante aplicação para o nosso método baseado em DGs. Graças a sua dedicação e competência, obtivemos nossas primeiras publicações durante o doutorado em conferência [66] e em revista [65].
} 


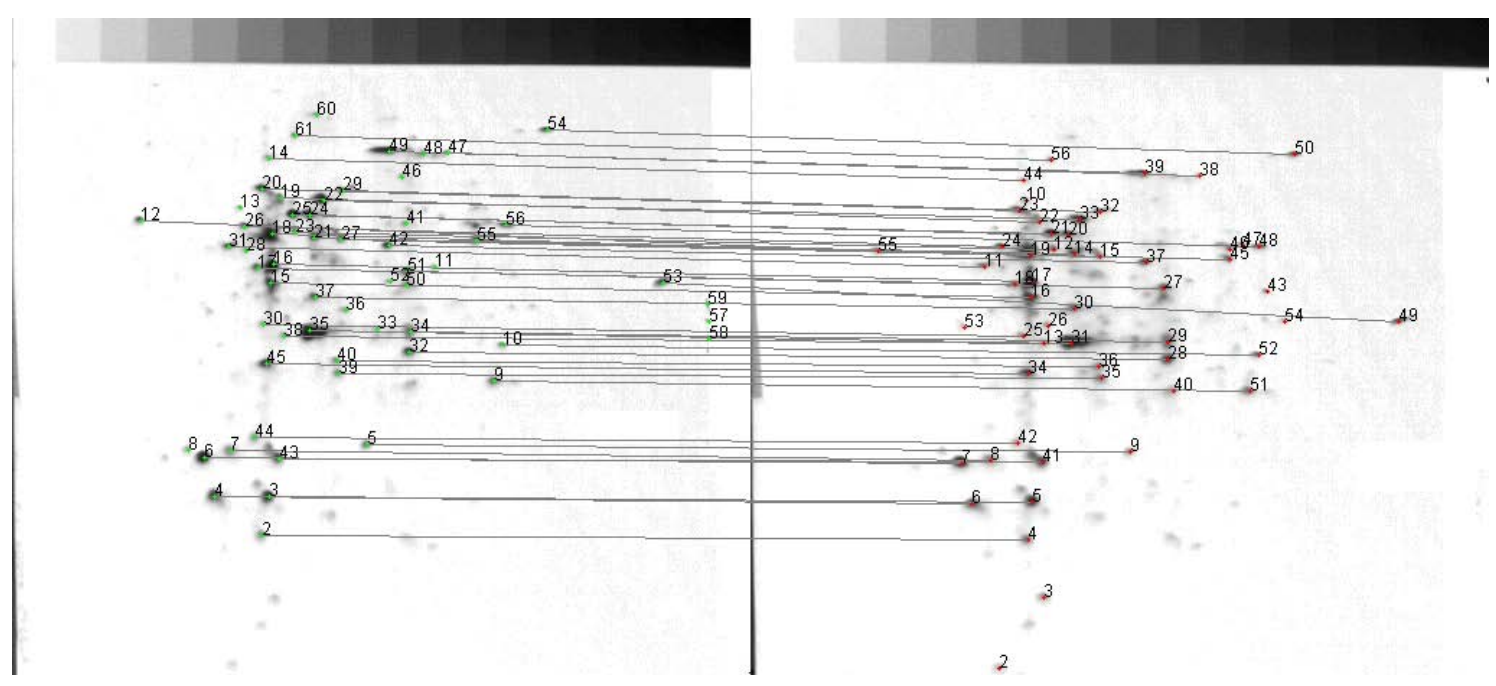

Figura 2.2: Correspondência entre um par de imagens 2DE.

pares de imagens para obtenção das mudanças protéicas. Para este estudo diferencial, é necessário registrar as duas imagens através do cálculo das correspondências entre os pontos detectados, representando as proteínas (Figura 2.2).

A técnica 2DE é bastante popular devido principalmente à sua simplicidade. Por outro lado, a simplicidade dos materiais e dos equipamentos utilizados não permitem um alto grau de controle durante os experimentos, de modo que, em geral, podem ocorrer grandes deformações nas imagens resultantes, dificultando ainda mais o processo de registro de imagens.

\subsubsection{Histórico}

Nesta seção, descrevemos os principais métodos relacionados ao casamento entre gels de eletroforese bidimensional (2DEM) [65, 66], exibindo as tendências principais de pesquisa.

Conforme descrito em [74], na prática, uma única imagem 2DE pode conter de 3000 a 4000 proteínas e estudos recentes realizaram análises diferenciais envolvendo conjuntos com até 100 imagens, exigindo algoritmos cada vez mais eficientes. Nesta Tese, propomos um algoritmo simples, baseado em DGs, com complexidade de tempo $O\left(n^{2}\right)$.

A maioria dos métodos existentes não considera a informação estrutural presente entre os pontos para o cálculo das correspondências. Geralmente, tais métodos se baseiam em 
características extraídas dos pontos para o cálculo das correspondências. Por exemplo, existem abordagens baseadas em distâncias entre pares de pontos correspondentes, como é o caso do algoritmo graduated assignment (GA) [29] usando a distância Euclidiana. Nesta mesma linha, podemos citar o algoritmo descrito em [9], combinando uma estratégia de casamento entre grafos bipartidos (BGM) e shape context (SC), cujos resultados são bastante conhecidos na literatura devido ao alto grau de robustez quanto à presença de outliers. Os dois métodos citados anteriormente alternam dois passos, cálculo das correspondências e estimativa das transformações, por exemplo, usando thin-plate splines (TPS), produzindo resultados bastante expressivos.

Um dos trabalhos mais importantes para o problema 2DEM é descrito em [74], onde há uma importante comparação entre diversos estados-da-arte, envolvendo algoritmos iterativos que alternam cálculo das correspondências e estimação de transformação. Estes dois passos são repetidos com o objetivo de refinar os resultados obtidos, seguindo a mesma estratégia do algoritmo ICP [13]. Durante os experimentos, os autores avaliaram diferentes tipos de distâncias e diferentes abordagens para o cálculo das correspondências. Por exemplo, dentre as distâncias avaliadas, podemos citar a Euclidiana e SC. Como abordagens para o cálculo das correspondências, os autores avaliaram diversos métodos, como por exemplo, closest point, $K$-closest points e BGM.

Seguindo esta tendência, aplicamos a formulação de atribuição quadrática definida na Seção 2.2, combinando SC no papel do termo linear e a distância estrutural (Equação 2.3) no papel do termo quadrático, resultando em uma função custo minimizada pelo algoritmo baseado em DGs, CAlculaMCS $\left(G_{i}, G_{m}\right)$, descrito na Seção 2.3.3, para solucionar o problema 2DEM. Para pares complexos de imagens 2DE, combinamos uma estratégia baseada em ICP, usando o algoritmo CALCulaMCSICP $\left(G_{i}, G_{m}\right)$, alternando cálculo das correspondências e estimação de deslocamento. Neste último, mostramos que é possível explorar o conhecimento a priori do usuário, fornecido através da validação de algumas poucas correspondências entre pontos, usadas como deslocamento incial para alinhar o grafo modelo sobre os pontos da entrada. Durante os experimentos, simulamos pares artificiais obtidos a partir de dados reais para comparar nossa abordagem com outras conhecidas na literatura, envolvendo distância Euclidiana, SC, BGM, estimação de transformação e ICP. Através dos experimentos artificiais, pudemos testar diferentes graus de degradação. Resultados envolvendo pares originais, obtidos em casos reais, também são 


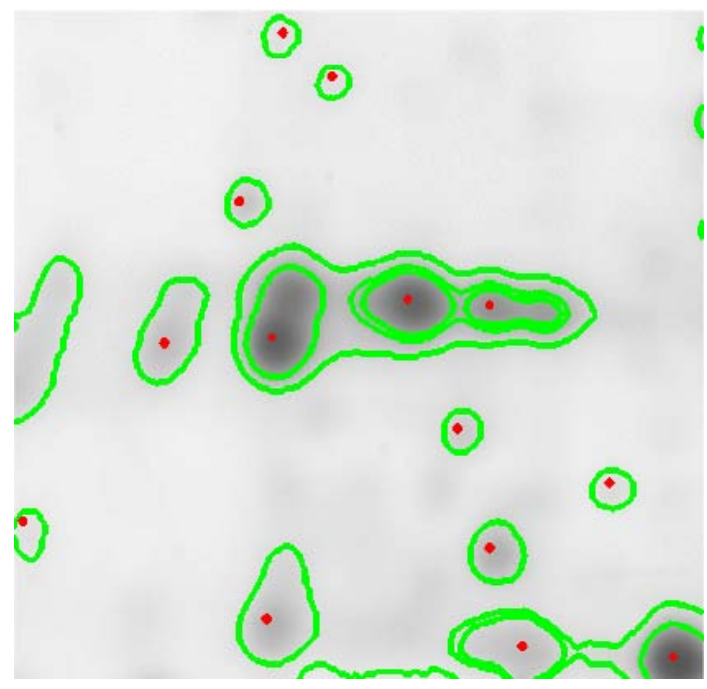

Figura 2.3: Detecção de proteínas. Curvas em verde ilustram bordas significativas. Pontos em vermelho indicam as posições com maiores concentrações protéicas.

exibidos para reforçar a qualidade e a aplicabilidade de nosso método.

\subsubsection{Detecção de proteínas}

Os melhores algoritmos de casamento de imagens 2DE são baseadas em técnicas de casamento de padrões de pontos, onde cada ponto representa uma proteína. Para a detecção das proteínas nas imagens 2DE, usamos o algoritmo descrito em [4], baseado na detecção de manchas 'significativas', em termos de contraste e de forma, neste caso, o formato oval ou elíptico. O ponto mais escuro na mancha é selecionado para representar cada proteína, representando o pico de concentração protéica. A Figura 2.3 ilustra um resultado de detecção obtido pelo algoritmo em questão.

\subsubsection{Distância entre shape context}

No papel do termo linear da Equação 2.1, usamos a mesma distância entre shape context (SC) usada no trabalho descrito em [74]. A idéia central de SC é descrever cada ponto em termos da distribuição dos pontos restantes em sua vizinhança, que é dividida em um conjunto de regiões, usando coordenadas polares. Um histograma é então obtido a partir 
do número de pontos contabilizados em cada região. O histograma normalizado relativo ao ponto $r$ e denotado por $h_{r}(k)$, onde $k$ identifica o número do bin dentro do histograma. Para avaliar a distância entre os SCs relativos a dois pontos, $r$ e $s$, usamos a distância $\chi^{2}$ :

$$
d_{A}(r, s)=d_{\chi^{2}}(S C(r), S C(s))=\frac{1}{2} \sum_{k} \frac{\left(h_{r}(k)-h_{s}(k)\right)^{2}}{h_{r}(k)+h_{s}(k)}
$$

\subsubsection{Experimentos}

Para exibir os benefícios do algoritmo proposto, comparamos nossa abordagem baseada em DGs com dois métodos bastante conhecidos na literatura: graduated assignment (GA) [29] e o algoritmo de casamento de grafos bipartidos (BGM) usando shape context (SC) [9], ambos explorando thin-plate splines (TPS) para estimação de transformação.

O BGM, usando o método Húngaro, é o método mais simples dentre os métodos usados para o casamento entre padrões de pontos, considerando apenas o termo linear da Equação 2.1 (veja [69] para mais detalhes). Dada uma matriz de dissimilaridade entre pontos, $C_{r, s}=d_{S C}(r, s)$, o objetivo é encontrar uma correspondência que minimize o custo dado por:

$$
\min _{Q_{r, s}} \sum_{r, s} C_{r, s} Q_{r, s}
$$

onde $Q_{r, s}$ representa uma matriz de permutações codificando o mapeamento. Para rejeitar possíveis outliers, um conjunto de pontos virtuais de custo $\varepsilon$ é incluído, expandindo a matriz de dissimilaridade $C_{\varepsilon}=\left[\begin{array}{ll}C & \varepsilon\end{array}\right]$. Usando-se dados com gabarito, os melhores resultados são definidos pela seleção de $\varepsilon^{*}$ que minimiza o número de erros.

Em [74], os métodos envolvidos foram testados de acordo com pares artificiais de padrões de pontos. Para cada par artificial, foram gerados dois conjuntos de pontos, denotados por source e target. O conjunto de pontos source foi criado de acordo com uma distribuição uniforme, e o target foi obtido como uma versão distorcida do source, simulando diferentes graus de deformações de acordo com transformações spline. No nosso caso, usando pares reais de imagens 2DE, aplicamos deformações nas duas imagens de cada par testado, usando ruído Gaussiano nas coordenadas dos pontos. Para simular a presença de outliers, os autores de [74] aleatoriamente adicionaram e removeram pontos dos conjuntos designados como target. No nosso caso, removemos aleatoriamente pontos das duas imagens do par, com o objetivo de aumentar ainda mais o grau de dificuldade. 

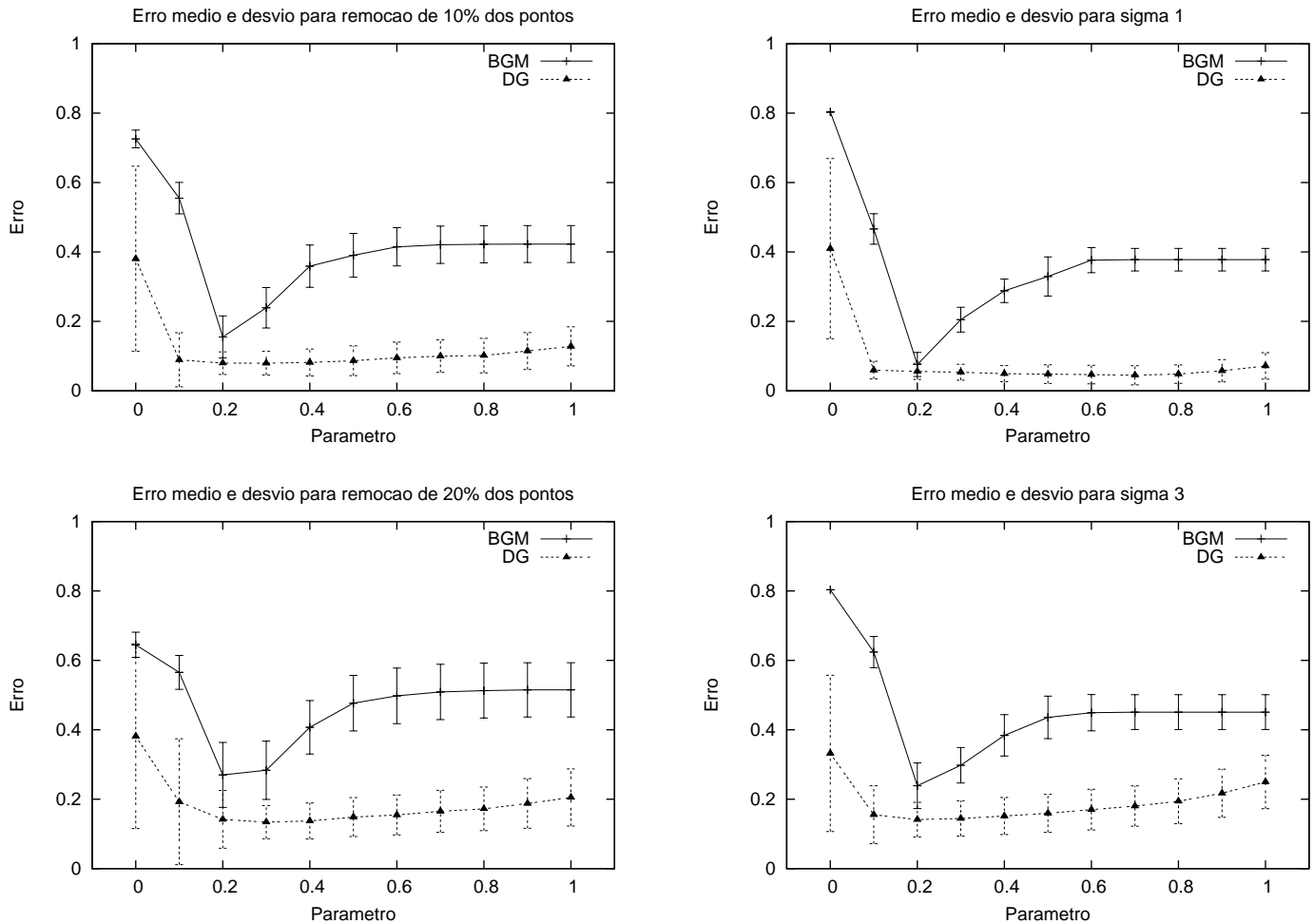

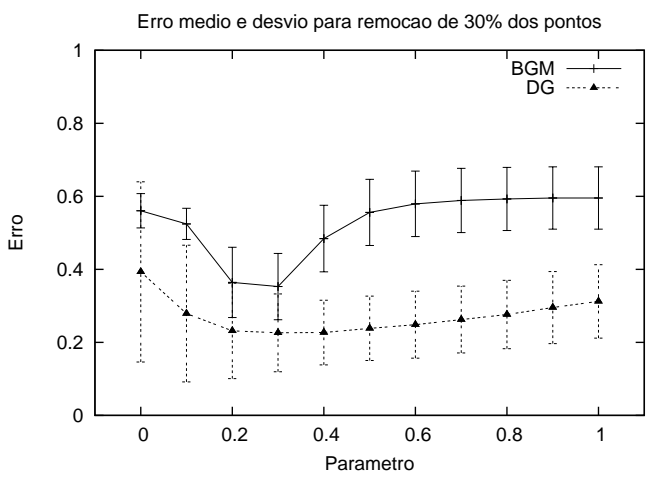

(a)

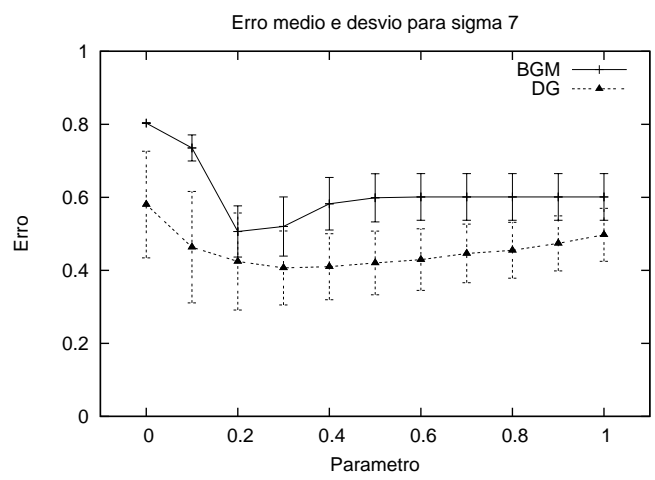

(b)

Figura 2.4: Experimento 1. Comparação entre nosso algoritmo DG e BGM usando o método Húngaro, para diferentes valores de $\lambda_{1}$ e $\varepsilon$, respectivamente. Resultados obtidos de acordo com (a) diferentes percentuais de pontos removidos e (b) diferentes graus de ruído Gaussiano. 
Para os gabaritos, imagens do banco de dados [2] foram processados, por exemplo, usando o método de detecção proposto em [4], e verificados manualmente. Nesta base de dados, cada imagem é numerada, por exemplo, o par "008-009" se refere às imagens "008" e "009".

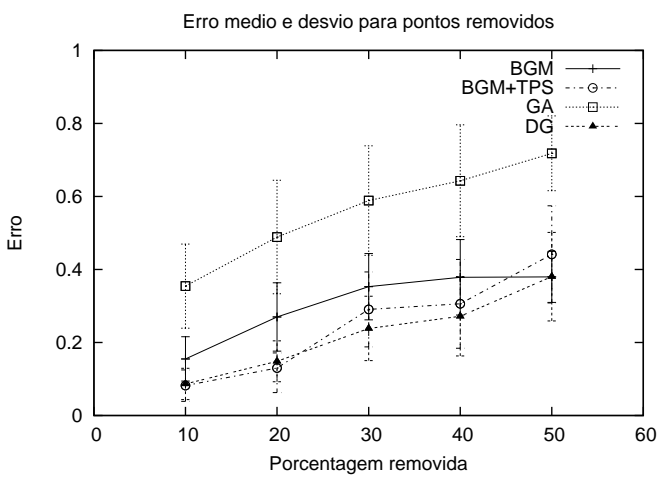

(a)

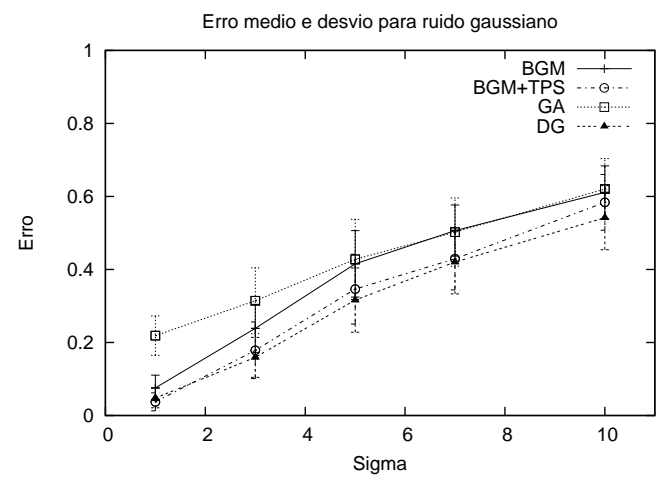

(b)

Figura 2.5: Comparação entre nossa abordagem DG, BGM, BGM+TPS e GA para (a) diferentes porcentagens de pontos removidos e (b) diferentes graus de ruído Gaussiano.

A avaliação computacional foi dividida em três experimentos. No primeiro, a abordagem DG é comparada com BGM para ilustrar o comportamento dos parâmetros $\lambda_{1}$ e $\varepsilon$. O objetivo do segundo experimento foi testar o método DG em situações mais desafiadoras, seguindo a mesma linha do primeiro experimento, mas aplicado a um complexo par de imagens 2DE, conforme ilustrado na Figura 2.6(a). Neste caso mais difícil, existe um número considerável de outliers e grandes deformações não-rígidas entre os pontos correspondentes. Isto resultou em falha de todos os métodos avaliados, exceto pela nossa abordagem baseada em DG, que demonstrou ser bastante promissora ao simular o uso do conhecimento a priori fornecido pelo usuário durante a inicialização dos deslocamentos $d_{x}^{0}$ e $d_{y}^{0}$, indicando que, através da validação de alguns pares de pontos correspondentes, o usuário pode obter bons resultados pelo nosso método. Do ponto de vista prático, isto é uma característica desejável, onde bons resultados devem ser obtidos com uma mínima intervenção por parte usuário. No terceiro e último experimento, resultados obtidos usando pares originais de [2] são exibidos na Tabela 2.1, ilustrando a aplicabilidade de nossa abordagem para situações reais, finalizando com uma análise de comportamento médio, seguindo os experimentos artificiais 1 e 2, mas aplicado para vários pares de imagens. 


\section{Experimento 1}

O primeiro experimento se baseou no par "008-009", ilustrado na Figura 2.2, onde o modelo se encontra à esquerda, com $\left|V_{m}\right|=61$. No lado direito da mesma figura, a entrada possui $\left|V_{i}\right|=56$ e existem 11 pontos da entrada sem correspondência. Este experimento foi dividido em duas partes:

Primeira parte: Subconjuntos de 10, 20 e 30\% foram aleatoriamente removidos dos dois conjuntos originais de pontos, de modo a comparar os métodos DG e BGM de acordo com um crescente número de outliers. Este cenário tem o objetivo de simular possíveis erros ocorridos durante a fase de detecção de proteínas e diferenças naturais de conteúdo protéico. Para cada porcentagem, 100 pares artificiais foram gerados e testados pelos dois métodos. Os erros de classificação foram computados para diferentes valores de $\lambda_{1}$ e $\varepsilon$. Em todos os experimentos, a taxa de erro foi calculada tomando-se o número de vértices da entrada classificados incorretamente, dividido pelo total $\left|V_{i}\right|$.

O parâmetro $\lambda_{1}$ na Equação 2.4 controla a importância entre aparência (SC) e estrutura. Para $\lambda_{1}=0$, somente a informação estrutural é considerada no cálculo do casamento. Similarmente, para $\lambda_{1}=1$, o algoritmo considera apenas a informação de SC. Já o parâmetro $\lambda_{2}$ é usado para balancear as duas penalidades geométricas presentes na Equação 2.3. Em todos os testes, usamos $\lambda_{2}=0.5$ de maneira que os dois termos, angular e modular, influenciem igualmente no resultado do casamento. Uma ilustração completa do primeiro experimento é apresentada na Figura 2.4(a).

Segunda parte: Para avaliar a robustez do algoritmo proposto devido a diferentes graus de deformações não-rígidas entre os pontos correspondentes, simulamos as deformações através da adição de ruído Gaussiano nas coordenadas dos pontos das duas imagens, de acordo com diferentes valores de desvio padrão $\sigma$. Valores elevados de $\sigma$ resultam em grandes perturbações nas coordenadas dos pontos. Neste caso, tanto a estrutura quanto a informação de SC são afetados pelo ruído Gaussiano. Para cada valor de $\sigma, 100$ pares artificiais foram gerados. O comportamento completo para esta parte do experimento é exibido na Figura 2.4(b).

Estas duas partes do experimento levaram à seguinte conclusão: as informações estruturais melhoraram os resultados de classificação, onde o nosso método DG obteve os melhores resultados, consistentes dentro de um grande intervalo de valores de $\lambda_{1}$, variando 
entre 0.2 e 0.8 .

Comparando-se os melhores resultados obtidos anteriormente, DG com $\lambda_{1}=0.5$ e BGM com $\varepsilon$ entre 0.2 e 0.3 , contra os métodos GA e BGM+TPS, aplicados ao mesmo conjunto de dados artificiais, nossa abordagem DG se manteve com os melhores resultados, conforme exibido na Figura 2.5. Em todos os experimentos, avaliamos GA e BGM+TPS usando a forma fechada de estimação de transformação via TPS, representada pela solução analítica dos parâmetros da transformação, usando a correspondência calculada entre os pontos. Na Figura 2.5(a), GA obteve um resultado ruim, indicando que a distância Euclidiana é uma característica pobre para discriminar padrões com poucos pontos e com uma quantidade expressiva de outliers nos dois conjuntos de pontos. Por outro lado, a informação de SC produziu melhores resultados. Nas Figuras 2.5(a) e (b), nossos resultados foram comparáveis aos resultados obtidos por BGM+TPS [9], conhecido na literatura por ser bastante robusto a presença de outliers.

\section{Experimento 2}

Neste experimento, ilustramos um cenário desafiador, onde os pares artificiais são obtidos a partir do par "095-098", que possui uma grande quantidade de outliers e fortes distorções não-rígidas entre os pontos correspondentes (Figura 2.6(a)). Na extrema direita da Figura 2.6(a), ilustramos um par artificial, resultante da aplicação de ruído Gaussiano usando $\sigma=5$, representando um caso mais complicado que o exemplo (Jitter $=4$ ) apresentado em [23]. Note que em nossos experimentos, simulamos distorções ainda mais fortes, usando $\sigma$ até 10 .

Os pares artificiais foram obtidos da mesma maneira que o experimento 1. Note que para o par "095-098", a grande quantidade de pontos 'espalhados' produz uma informação extremamente pobre de SC. Neste caso, mesmo a abordagem robusta BGM+TPS não conseguiu obter resultados satisfatórios, conforme ilustrado na Figura 2.6(b). Observamos também resultados bastante pobres de nosso método quando aplicamos a inicialização automática do alinhamento, baseada nas medianas dos dois conjuntos de pontos, conforme descrito no final da Seção 2.3.3. Estes resultados são ilustrados pela curva DG1 na Figura 2.6(b).

Para testar a aplicabilidade de nosso método neste complexo cenário, simulamos a 

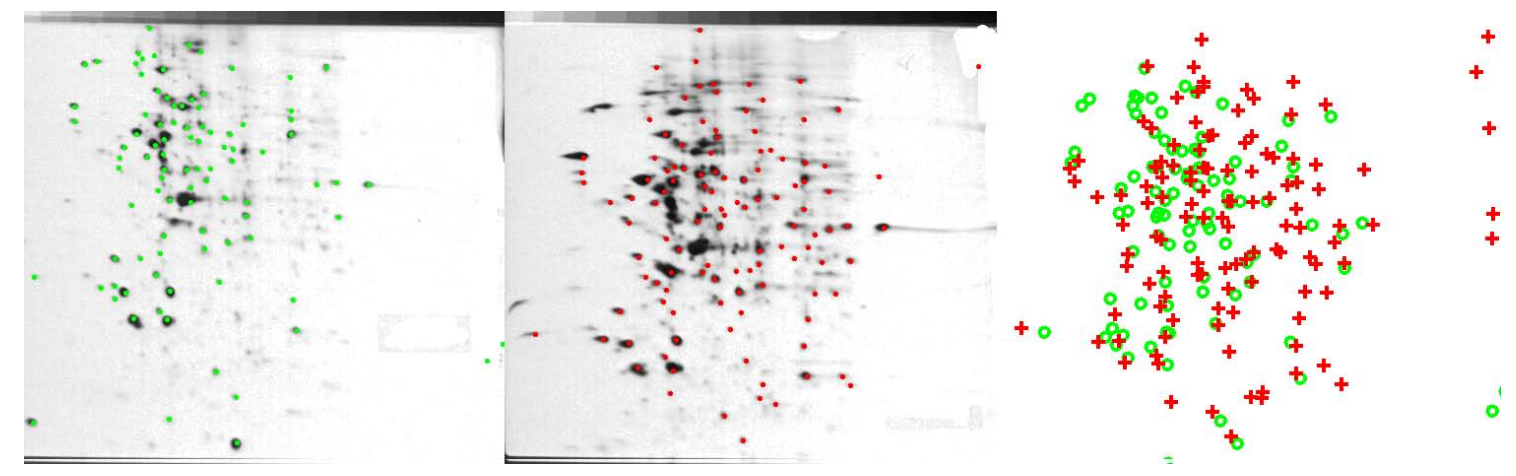

(a)
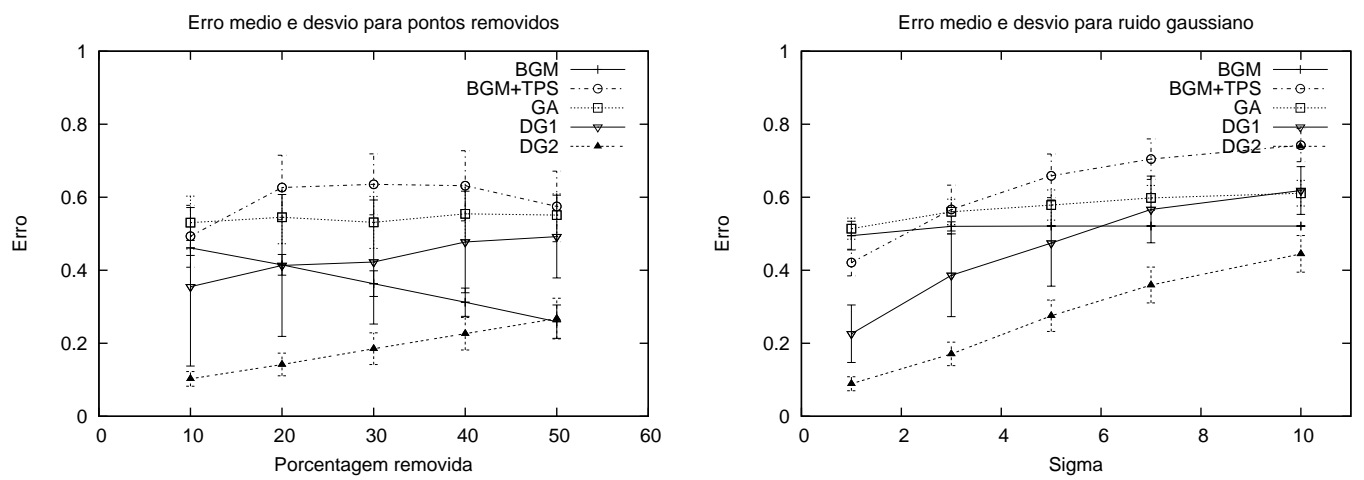

(b)

Figura 2.6: Comparação, usando (a) par complexo "095-098": grande quantidade de outliers e fortes deformações não-rígidas. O modelo se encontra à esquerda com $\left|V_{m}\right|=95$. Ao centro, a entrada possui $\left|V_{i}\right|=119$. Neste cenário complexo, existem 57 pontos da entrada sem correspondência. Na extrema direita, um exemplo de ruído Gaussiano $(\sigma=5)$ aplicado nos dois conjuntos de pontos, sobrepostos para uma melhor vizualização. (b) Comparação entre as abordagens DG, BGM, BGM+TPS, GA, considerando 100 pares artificiais obtidos de acordo com diferentes porcentagens de pontos removidos e diferentes valores de desvio padrão para ruído Gaussiano. A curva DG2 representa o algoritmo proposto simulando o conhecimento a priori fornecido pelo usuário. 
inclusão do conhecimento a priori dado pelo usuário, inicializando o deslocamento inicial $d_{x}^{0}$ e $d_{y}^{0}$ com a mediana dos deslocamentos entre os pontos correspondentes usando o gabarito. Em uma situação real, esta informação poderia ser obtida a partir de algumas correspondências fornecidas pelo usuário, validando alguns pares de pontos correspondentes para inicializar os valores de $d_{x}^{0}$ e de $d_{y}^{0}$. Como resultado desta simulação, obtivemos sensíveis melhorias, conforme exibido na Figura 2.6(b), onde a curva DG2 superou todos os outros métodos.

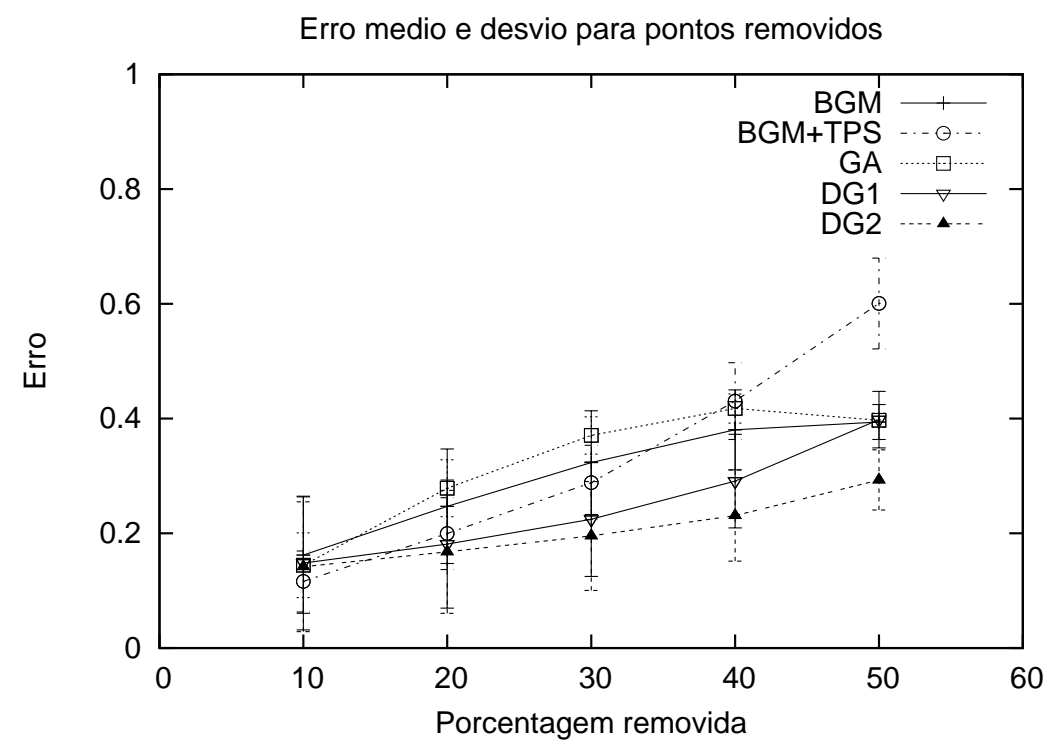

Figura 2.7: Comportamento médio dos métodos avaliados, usando 4500 pares artificiais, obtidos a partir de 45 pares originais, usando os marcadores de [2].

\section{Experimento 3}

A Tabela 2.1 exibe resultados envolvendo pares originais de imagens obtidas de [2]. Em todos os casos, nossa abordagem DG superou todos os outros métodos envolvidos, BGM, BGM+TPS e GA. A diferença na qualidade de resultado é maior para o caso difícil, representado pelo par "095-098". Diferentemente do experimento 2, para todos os pares originais testados, incluindo o par "095-098", não usamos nenhuma informação de gabarito, ou seja, a inicialização dos deslocamentos foi feita de maneira automática, usando somente as medianas das coordendas dos dois conjuntos de pontos, atribuindo 


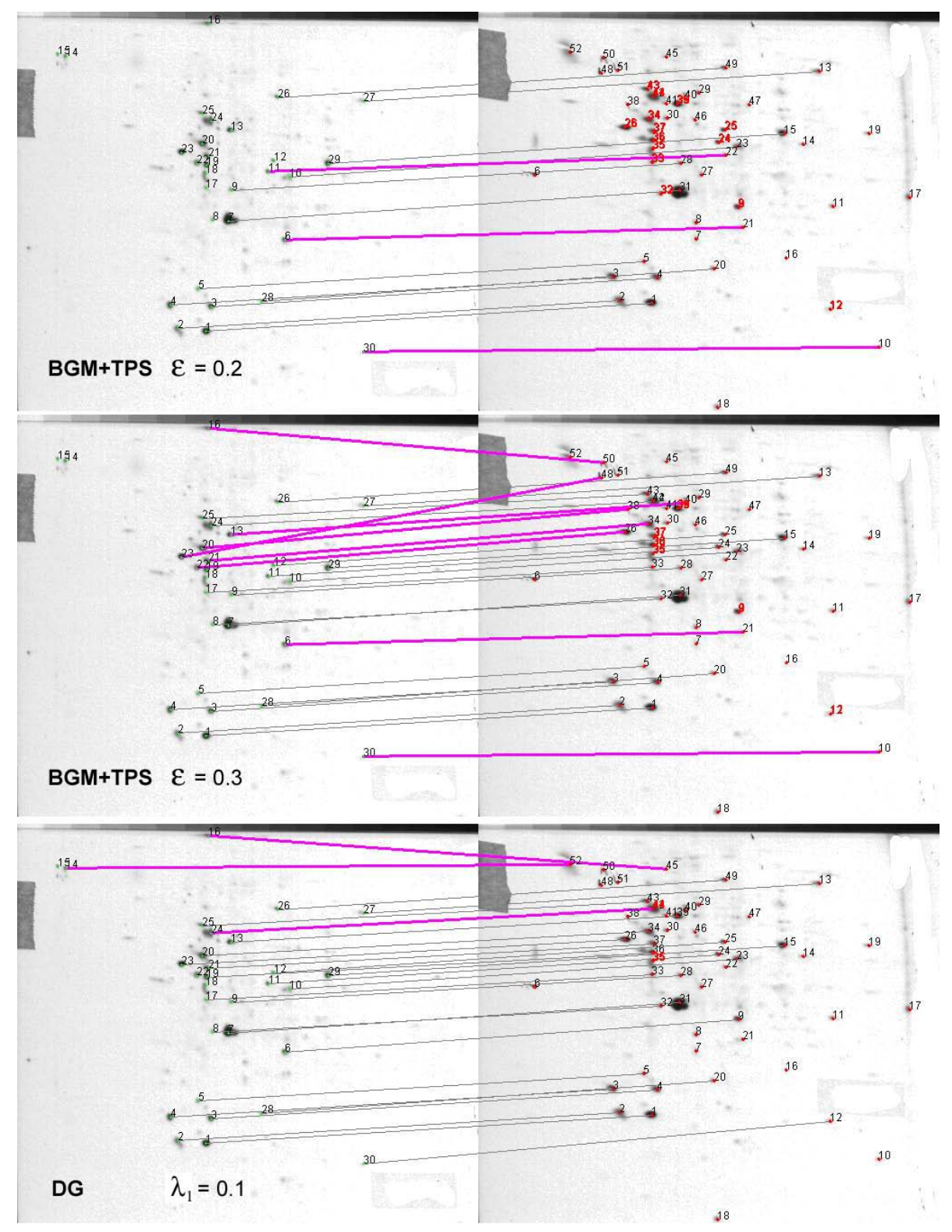

Figura 2.8: Par "031-032": um exemplo em que nossa abordagem DG supera o algoritmo BGM+TPS. 1a. linha: para valores pequenos de $\epsilon$, um número excessivo de pontos da entrada são incorretamente descartados como ouliters pelo método BGM+TPS. Os ouliters são representados pelos números em destaque. 2a. linha: para altos valores de $\epsilon$, BGM+TPS produz correspondências incorretas, indicadas pelas linhas destacadas, sugerindo que a forma fechada de TPS é inadequada para este caso. 3a. linha: nosso resultado. 
Tabela 2.1: Comparação entre as abordagens DG, BGM, BGM+TPS e GA, usando os pares originais de [2]. Para cada método, são exibidos o parâmetro utilizado e a correspondente taxa de erro.

\begin{tabular}{c|cc|cc|c|cc}
\hline imagens & $\varepsilon$ & BGM & $\varepsilon$ & BGM+TPS & GA & $\lambda_{1}$ & DG \\
\hline $006-007$ & 0.3 & 0.1111 & 0.2 & $\mathbf{0 . 0 0 0 0}$ & 0.1667 & 0.5 & $\mathbf{0 . 0 0 0 0}$ \\
$008-009$ & 0.2 & 0.0492 & 0.2 & 0.0179 & 0.1475 & 0.5 & $\mathbf{0 . 0 1 6 4}$ \\
$011-012$ & 0.3 & 0.0851 & 0.2 & 0.1220 & 0.2927 & 0.5 & $\mathbf{0 . 0 2 4 4}$ \\
$014-015$ & 0.2 & 0.3395 & 0.2 & 0.2970 & 0.1881 & 0.7 & $\mathbf{0 . 1 6 8 3}$ \\
$016-017$ & 0.2 & 0.3684 & 0.2 & 0.3878 & 0.5921 & 0.3 & $\mathbf{0 . 2 2 3 7}$ \\
$019-020$ & 0.2 & 0.0213 & 0.2 & 0.0638 & 0.2340 & 0.6 & $\mathbf{0 . 0 1 4 5}$ \\
$028-029$ & 0.2 & 0.2755 & 0.2 & 0.2959 & 0.3776 & 0.3 & $\mathbf{0 . 1 6 4 8}$ \\
$031-032$ & 0.3 & 0.1539 & 0.3 & 0.2692 & 0.2667 & 0.1 & $\mathbf{0 . 0 9 6 2}$ \\
$074-075$ & 0.2 & 0.1563 & 0.2 & 0.2099 & 0.1875 & 0.5 & $\mathbf{0 . 0 6 1 7}$ \\
$095-098$ & 0.2 & 0.4874 & 0.2 & 0.4632 & 0.6387 & 0.5 & $\mathbf{0 . 1 0 9 2}$ \\
\hline
\end{tabular}

$d_{x}^{0}=\operatorname{median}\left\{x_{i}\right\}-\operatorname{median}\left\{x_{m}\right\}$ e $d_{y}^{0}=\operatorname{median}\left\{y_{i}\right\}-\operatorname{median}\left\{y_{m}\right\}$, conforme descrito no fim da Seção 2.3.3.

Como um último teste, avaliamos o comportamento médio de cada método, usando 21 marcadores de [2], pertencentes a dez imagens diferentes $(6 \ldots 9,12,14 \ldots 17,19)$, resultando em 45 combinações possíveis de pares de imagens. Para testar a presença de outliers, seguimos a mesma estratégia dos experimentos 1 e 2 , removendo diferentes porcentagens de pontos e gerando 100 pares artificiais para cada combinação, totalizando 4500 pares. Na Figura 2.7, observamos que para um crescente número de pontos removidos, a taxa de erro de todos os métodos aumenta devido ao crescente número de outliers. Note que a curva DG2 permanece mais estável, superando os demais métodos.

A Figura 2.8 ilustra o par "031-032", onde nosso método DG supera o algoritmo BGM+TPS, indicando que, para o problema 2DEM, a avaliação estrutural usando DGs é mais adequada que a forma fechada de estimação de transformação usando TPS, principalmente na presença de outliers, comum em situações reais. As Figuras 2.9 até 2.11 ilustram os resultados obtidos pela nossa abordagem sobre os pares restantes, usados na Tabela 2.1. 


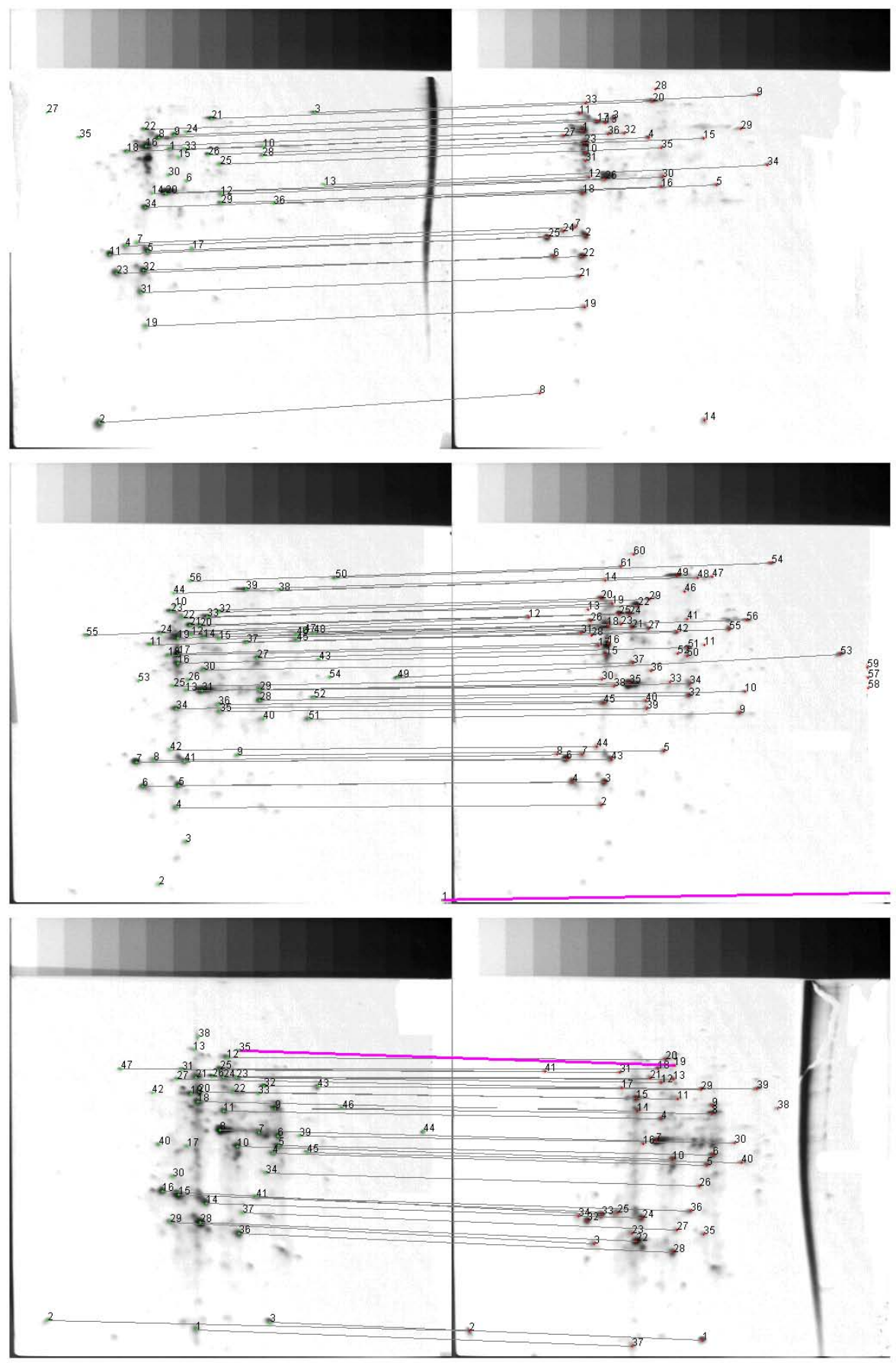

Figura 2.9: Nossos resultados para os pares "006-007", "008-009", "008-009". 

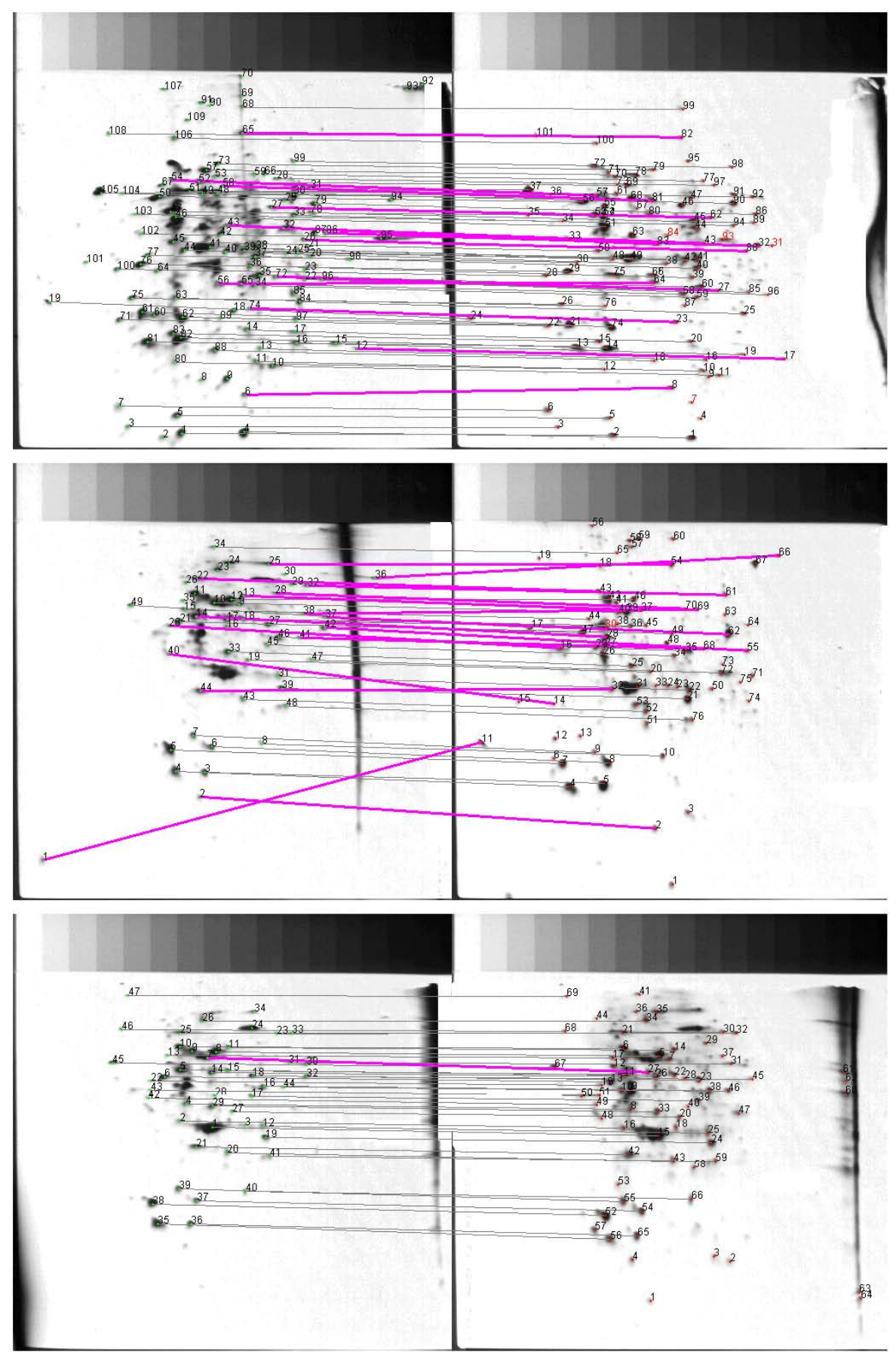

Figura 2.10: Nossos resultados para os pares "014-015", "016-017", "019-020". 

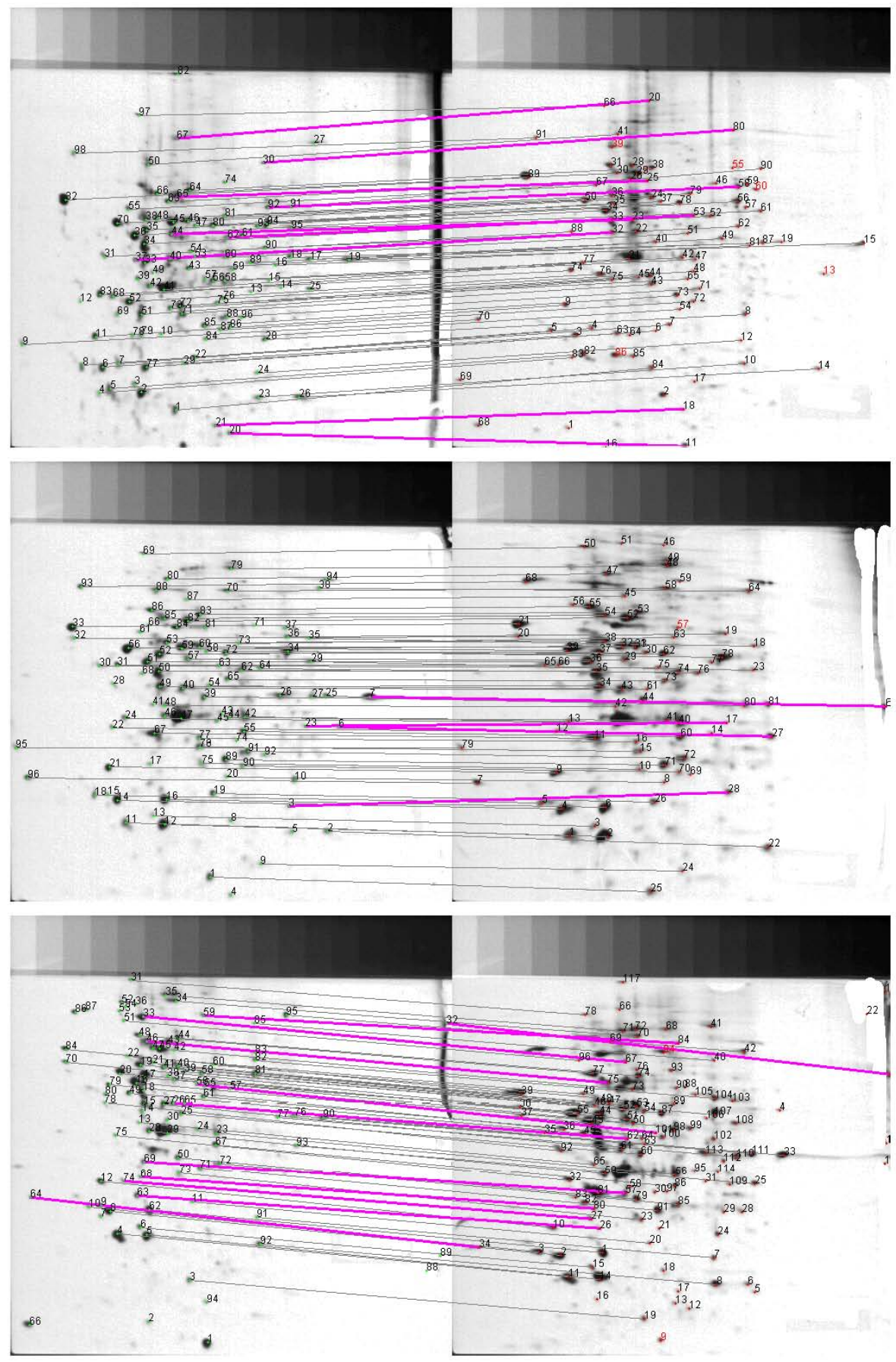

Figura 2.11: Nossos resultados para os pares "028-029", "074-075", "095-098". 


\subsection{Aplicação 2: segmentação interativa de imagens naturais}

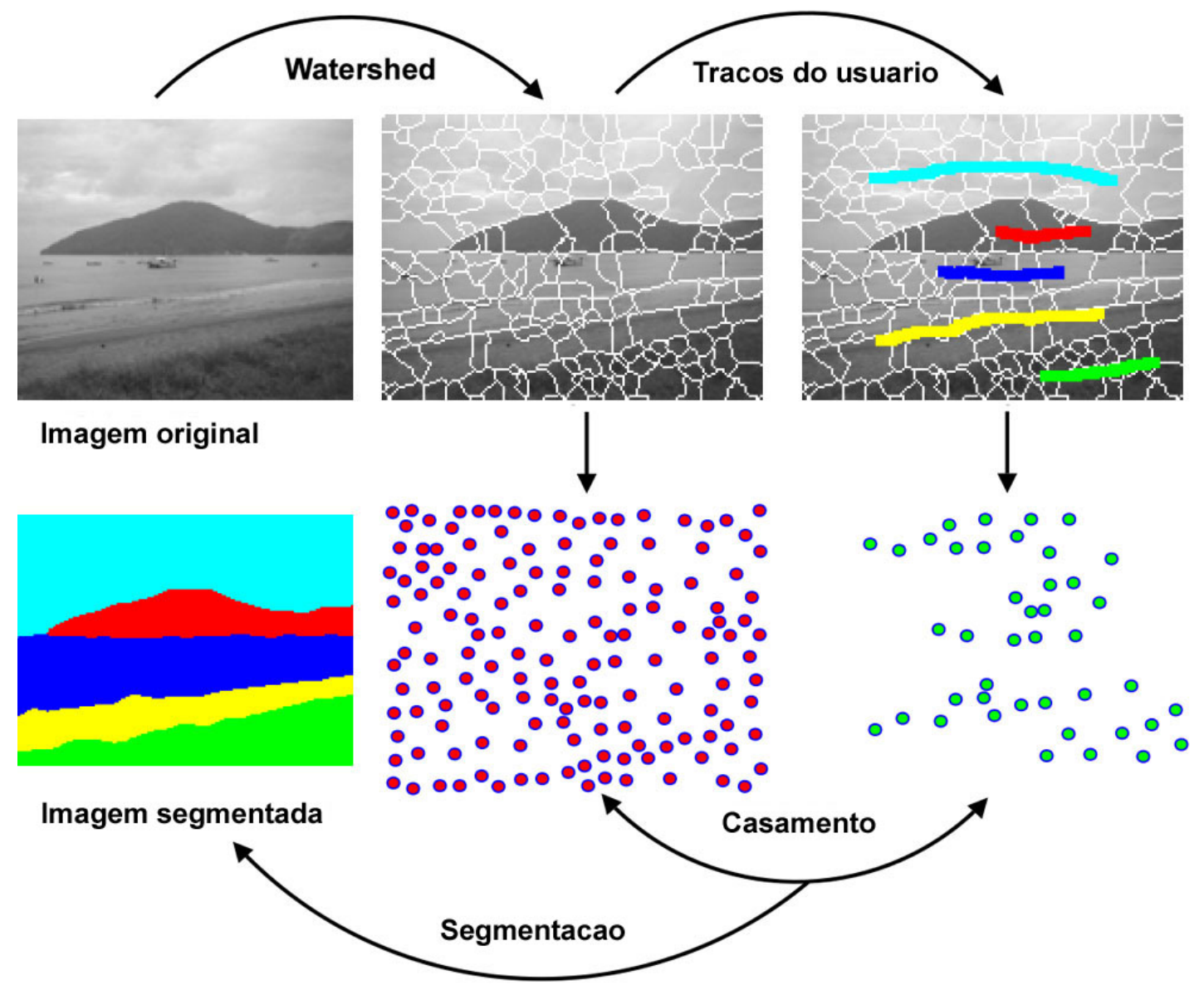

Figura 2.12: Resumo de nossa abordagem. Primeiramente, dividimos a imagem de entrada em regiões, via watershed. A seguir, dois padrões de pontos são gerados, um incluindo todas as regiões, e outro incluindo somente os pontos representando as regiões marcadas pelo usuário. Finalmente, o resultado da segmentação é obtido através do casamento entre os padrões de pontos.

A segmentação de imagens naturais é um problema fundamental em processamento de imagens. Basicamente, o objetivo é dividir uma dada imagem em diferentes regiões. Um exemplo de aplicação é a extração do objeto de interesse, removendo-se o antigo fundo para uma composição com um novo fundo de imagem. Para o caso geral, existe 
Casamento de padrões de pontos via casamento entre grafos

ambiguidade de interpretação dos objetos de interesse, sendo necessária uma intervenção do usuário no processo de segmentação. Uma maneira bastante comum de interação com o usuário é através de traços sobre a imagem, rotulando as regiões de interesse. A idéia é que após esta rotulação inicial, o processo de segmentação siga automaticamente para o restante da imagem. O sistema deve também permitir adicionar e remover traços para obter o resultado desejado, com um mínimo de esforço.

Aplicamos nossa abordagem baseada em DG para o cálculo eficiente de uma solução inicial para o problema de segmentação interativa de imagens, estendendo um trabalho anterior, descrito em $[31]^{2}$. Combinado a um simples pós-processamento, usado para melhorar a qualidade da segmentação, nosso método DG reduziu, em muitos casos, a intervenção do usuário, no sentido em que poucos traços resultaram em segmentações de qualidade.

Apesar da existência de vários sistemas de segmentação baseados em grafos, não é comum o uso de técnicas de casamento entre grafos. No nosso caso, dado um grafo de entrada, representando a imagem, e um grafo modelo, representando as regiões marcadas pelos usuário, o objetivo é encontrar um homomorfismo entre os dois grafos, mapeando vértices da entrada a vértices do modelo. Nossa abordagem é resumida na Figura 2.12.

\subsubsection{Histórico}

Nesta seção, descrevemos os principais métodos baseados em grafos relacionados ao problema de segmentação, considerando as intenções do usuário, expressas tipicamente através de traços fornecidos no início do processo de segmentação.

O trabalho pioneiro envolvendo graph cuts (GC) foi descrito em [17] por Boykov e Jolly, usando o algoritmo, baseado em pixels, de corte-mínimo/fluxo-máximo para a extração de objeto/fundo. Os traços fornecidos pelo usuário são usados como hard constraints e para coletar informações estatísticas sobre as cores dos pixels. O algoritmo Grabcut [76] é uma extensão deste método, simplificando a interação do usuário, explorando misturas de

\footnotetext{
${ }^{2}$ Esta parte da pesquisa foi realizada em colaboração com os professores Luís Augusto Consularo e Isabelle Bloch, e com a aluna de doutorado Ana Beatriz Vicentim Graciano. Em particular, a implementação do algoritmo descrito em [31], disponibilizada pelo professor Consularo, foi o ponto de partida para os nossos trabalhos, de onde surgiu o nosso primeiro protótipo do algoritmo baseado em DGs aplicado ao problema de segmentação.
} 
Gaussianas para as estatísticas das cores dos pixels. Desde então, vários outros métodos baseados em GC foram propostos na literatura, estendendo para a segmentação de vídeos, através de formulações matemáticas cada vez mais sofisticadas.

Um outro trabalho bastante importante envolvendo segmentação baseada em pixels é descrito em [46]. O problema é tratado por passeios aleatórios (random walks). Para cada pixel, associa-se o rótulo de maior probabilidade de um caminhante aleatório atingí-lo, partindo do correspondente traço fornecido pelo usuário. Um trabalho recente relacionado com passeios aleatórios e GC é descrito em [35], onde o problema de segmentação é tratado como uma inferência estatística de transdução.

Algoritmos baseados em pixels são fundamentais para o cálculo de resultados precisos de segmentação, especialmente na presença de bordas 'fracas' ou de pouco contraste. Apesar disso, recentemente, abordagens baseadas em fusão de regiões têm sido propostas na literatura. Li et al. [55] apresentaram um método de fusão de regiões, combinando GC e regiões de watershed. Ning et al. [62] propuseram um mecanismo de fusão de regiões por similaridade maximal para o problema de segmentação interativa. Comparadas com as abordagens baseadas em pixels, os autores observaram que as estratégias baseadas em regiões aceleram o processo de segmentação, além de aumentar a robustez com relação a ruídos. Nosso método é baseado em fusão de regiões para obter uma segmentação inicial, refinada por um pós-processamento baseado em pixels, combinando as vantagens das duas abordagens.

Nossos experimentos foram inspirados no importante trabalho de Bai e Sapiro [7], onde os autores apresentaram resultados impressionantes em termos de qualidade e de simplicidade de traços. O método proposto foi baseado em fast kernel density estimation [96] para as estatísticas de cores, melhorando a qualidade dos resultados obtidos anteriormente por Protiere e Sapiro [72], usando distância geodésica. Neste trabalho, tentamos seguir a mesma simplicidade de traços apresentada em [7].

\subsubsection{Abordagem de segmentação interativa}

O problema de segmentação interativa de imagens naturais (INIS) [1] é uma tarefa mais simples que o problema 2DEM, no sentido em que os dois padrões de pontos já estão alinhados e o método ICP não é necessário neste caso. Neste cenário mais simples, propomos 


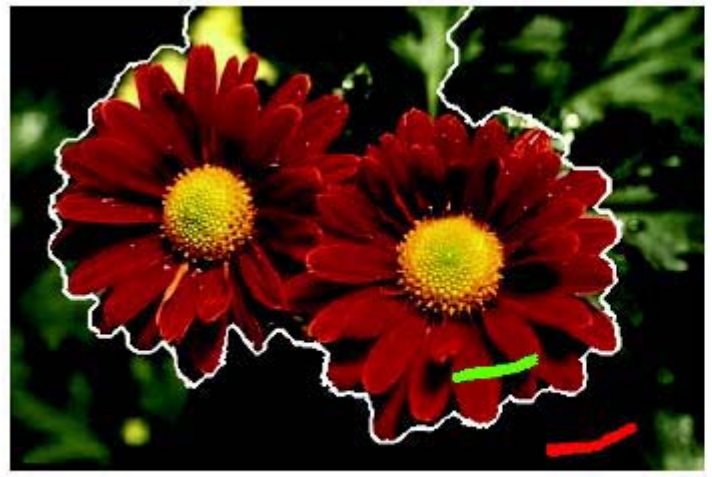

(a)

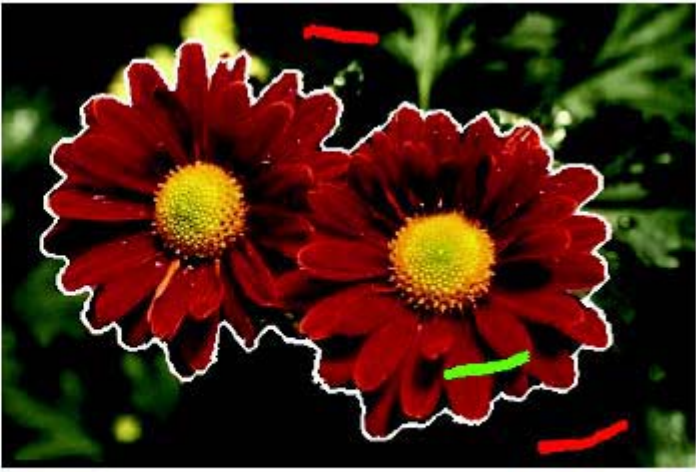

(b)

Figura 2.13: (a) Resultado obtido com dois traços, incorretamente incluindo parte do fundo. (b) Adição de um terceiro traço para eliminar completamente o fundo, corrigindo a extração das flores. Os contornos das regiões obtidas estão destacados.

um método de segmentação, com as seguintes características:

1. O algoritmo é baseado na transformação watershed [93]. Primeiramente, aplicamos o watershed na imagem de entrada. Cada região resultante é representada pelo seu centróide. O grafo de entrada inclui todas as regiões, enquanto o grafo modelo inclui apenas as regiões marcadas pelos traços do usuário. Note que o uso de regiões oferecem importantes vantagens em relação ao uso de pixels: regiões são mais tolerantes a ruídos, suas bordas coincidem com as bordas dos objetos, além de facilitar a formulação matemática do problema, por exemplo, envolvendo relações espaciais entre as regiões $[83,84]$.

2. O resultado inicial de segmentação é dado pelo casamento entre os dois grafos, mapeando cada vértice de entrada a um vértice do modelo (homomorfismo, muitos-paraum), usando o algoritmo CalculaHomomorfismo $\left(G_{i}, G_{m}\right)$ definido na Seção 2.3.3.

3. A segmentação inicial é posteriormente refinada no nível de pixels, removendo-se as regiões desconexas e melhorando a borda dos objetos de interesse (veja Seção 2.5.4).

4. O método proposto é computacionalmente mais rápido que o algoritmo proposto em [31]. Reduzimos a complexidade de tempo em uma ordem de magnitude e a qualidade dos resultados podem ser comparados a muitos algoritmos do estado-daarte. 
Apesar de o nosso algoritmo não ser limitado apenas à segmentação binária, objeto/fundo, nossos experimentos se concentram em exemplos de extração de objetos para composição de imagens com um novo fundo. No caso da segmentação binária, o algoritmo trabalha com duas 'cores' para os traços do usuário, uma cor representando o objeto e outra representando o fundo. Durante o início do processo de segmentação, o usuário é requisitado a marcar as regiões de interesse, usando as duas cores, transmitindo ao algoritmo informações sobre o objeto e o fundo. O objetivo do algoritmo é propagar a rotulação inicial para o restante da imagem. No nosso caso, exploramos as cores das regiões como informação de aparência, e as posições relativas entre as regiões como informação estrutural.

Nosso método permite ao usuário adicionar e remover traços de modo a obter a segmentação desejada. A Figura 2.13(a) exibe uma segmentação binária calculada a partir dos traços iniciais fornecidos pelo usuário. Na Figura 2.13(b), o usuário adiciona um novo traço de maneira a corrigir o resultado de segmentação. Note que após adicionar ou remover um traço, o grafo modelo é atualizado e o algoritmo para o cálculo de casamento é executado usando o novo modelo.

\subsubsection{Distância entre cores}

Além de permitir o uso das relações espaciais entre as regiões, os traços são utilizados também para coletar informações sobre as cores das regiões. Como informação de aparência, usamos as intensidades médias de cada região. Para imagens coloridas, usamos três médias, uma para cada canal no espaço de cores CIELAB. Para cada vértice $v$, o seu atributo $\mu(v)$ é composto pelos três valores médios. Para um dado par $\left(v_{i}, v_{m}\right)$, comparamos diretamente os respectivos atributos dos vértices:

$$
d_{A}\left(v_{i}, v_{m}\right)=\frac{\text { EuclideanDistance }\left(\mu\left(v_{i}\right), \mu\left(v_{m}\right)\right)}{C_{A}} .
$$

avaliando a distância Euclidiana entre os atributos $\mu\left(v_{i}\right)$ and $\mu\left(v_{m}\right)$, no papel do termo linear da Equação 2.1. O resultado final é normalizado por uma constante $C_{A}$ para manter os valores entre 0 e 1. 

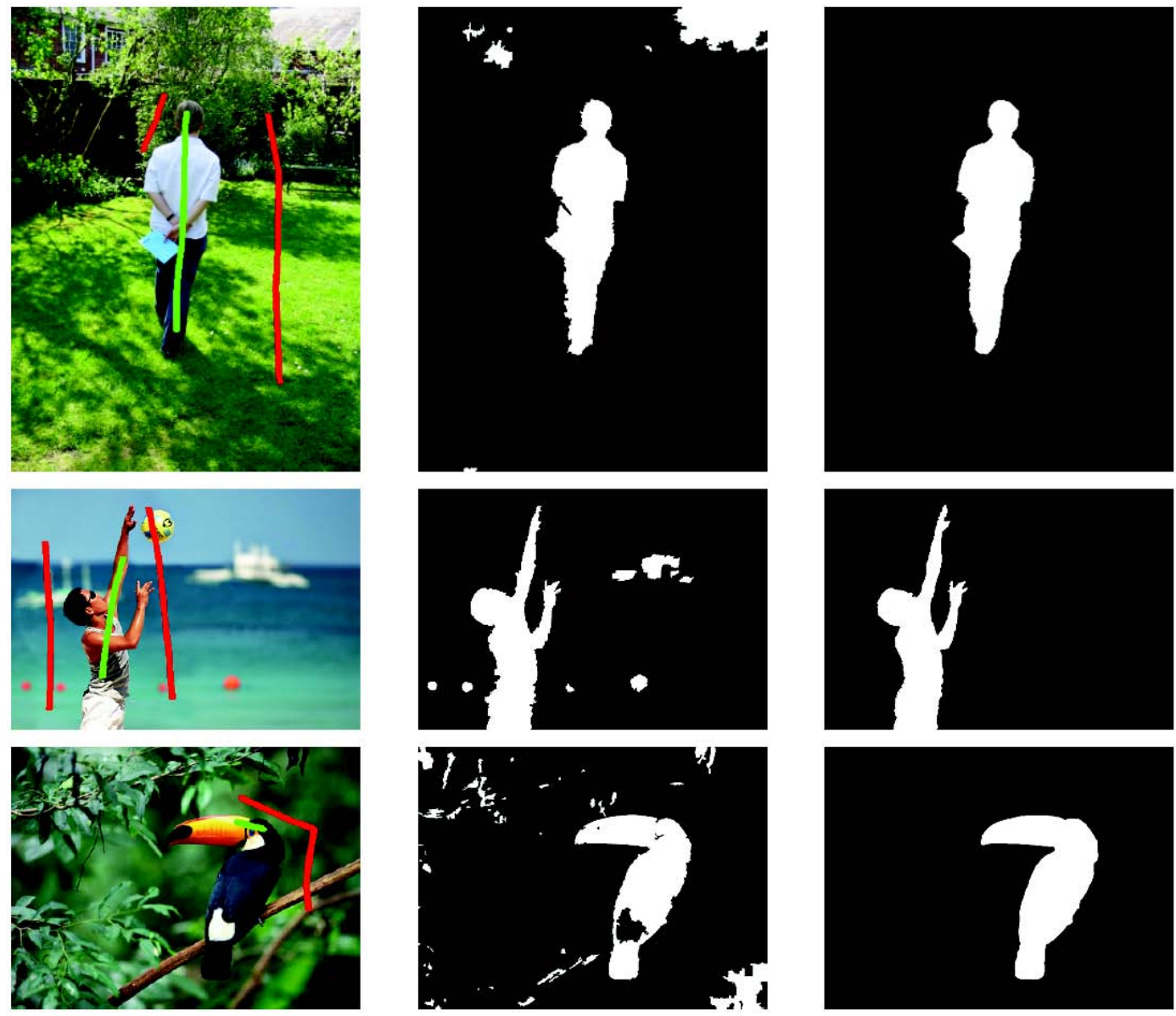

Figura 2.14: Exemplos de segmentação usando imagens de Grabcut [76], Bai e Sapiro [7], e o 'tucano' usado em [62]. Coluna esquerda: imagens originais com traços do usuário. Coluna do meio: regiões rotuladas, sem o pós-processamento. Coluna direita: resultado após o pós-processamento.

\subsubsection{Pós-processamento}

Após computar o resultado inicial de segmentação, a nossa abordagem refina o resultado no nível de pixels, similarmente ao algoritmo descrito em [55], aproveitando as vantagens das duas técnicas, fusão de regiões e segmentação baseada em pixels, em termos de velocidade, robustez a ruídos e precisão nas bordas.

Para aumentar a qualidade dos resultados de segmentação e simplificar os traços necessários providos pelo usuário, aplicamos os seguintes passos durante o pós-processamento:

(P1) Os traços são impostos como hard constraints, como por exemplo em [17]. 
(P2) As regiões resultantes da segmentação inicial são processadas de maneira a garantir que cada região esteja conectada com um traço do usuário de mesma cor. Por exemplo, regiões classificadas como objeto que não estão conectadas a um traço de objeto são fundidas com as regiões representando o fundo. Similarmente para as regiões classificadas como fundo.

(P3) Refinamento no nível de pixels nas bordas do objeto de interesse.

(P4) Filtros conexos [34] são aplicados para suavizar as bordas do objeto.

Todos os passos acima são procedimentos simples que podem ser executados em complexidade de tempo linear. Cada passo é detalhado a seguir.

O passo (P1) indica que cada pixel colorido por um traço do usuário deve manter este rótulo ou cor no resultado final de segmentação. No nosso caso, usamos esta restrição para contornar o problema de bordas fracas, de pouco ou sem contraste.

O passo (P2) é extremamente importante e implica que o resultado final da segmentação possui no máximo uma componente conexa por traço desenhado pelo usuário. Durante os experimentos, observamos que este passo agregou robustez ao algoritmo, exigindo uma quantidade menor de traços para obter bons resultados de segmentação (Figura 2.14). Note que em [7], pode-se provar por desigualdade triangular que a métrica utilizada pelos autores induz resultados de segmentação em que cada traço resulta em no máximo uma componente, reforçando ainda mais a necessidade deste passo para diminuir o esforço do usuário.

O passo (P3) tem o objetivo de corrigir pixels mal classificados presentes nas bordas dos objetos de interesse, devido a imprecisões no cálculo do watershed, causadas principalmente pelo descarte da informação de cores. A princípio, este passo pode ser executado por um algoritmo baseado em pixels, usando traços gerados automaticamente a partir de uma estreita faixa contornando o objeto de interesse. No nosso caso, usamos uma heurística simples que resultou em resultados bastante satisfatórios. Primeiramente, todos os pixels contidos na faixa são marcados como desconhecidos, considerando uma estreita faixa formada pelas bordas, interna e externa, do objeto. O objetivo é reclassificar cada pixel $r$ contido na faixa, atribuindo um rótulo associado ao pixel de cor mais próxima, avaliando todos os pixels em um círculo de raio $R$ e centro em $r$, usando a Equação 2.8. Este passo 
é repetido $K$ vezes. Em nossos experimentos, usamos $R=10$ pixels e repetimos este passo $K=10$ vezes.

O passo (P4) aplica um filtro conexo na borda do objeto de modo a suavizar o seu contorno. Isto é feito através de uma sequência de operadores morfológicos, mais precisamente de aberturas e de fechamentos [34] usando os elementos estruturantes exibidos na Figura 2.15.
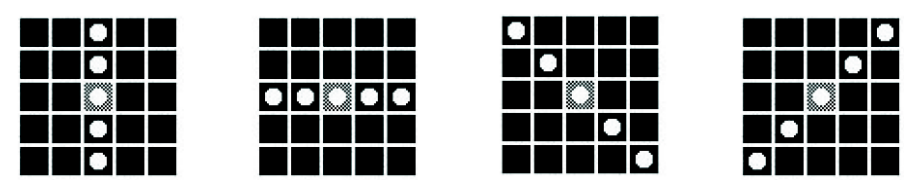

Figura 2.15: Elementos estruturantes usados para suavizar as bordas do objeto de interesse.

A Figura 2.14 ilustra as melhorias produzidas pelos passos de pós-processamento, convertendo o resultado inicialmente fragmentado em um resultado mais limpo e com contornos mais lisos.

\subsubsection{Experimentos}
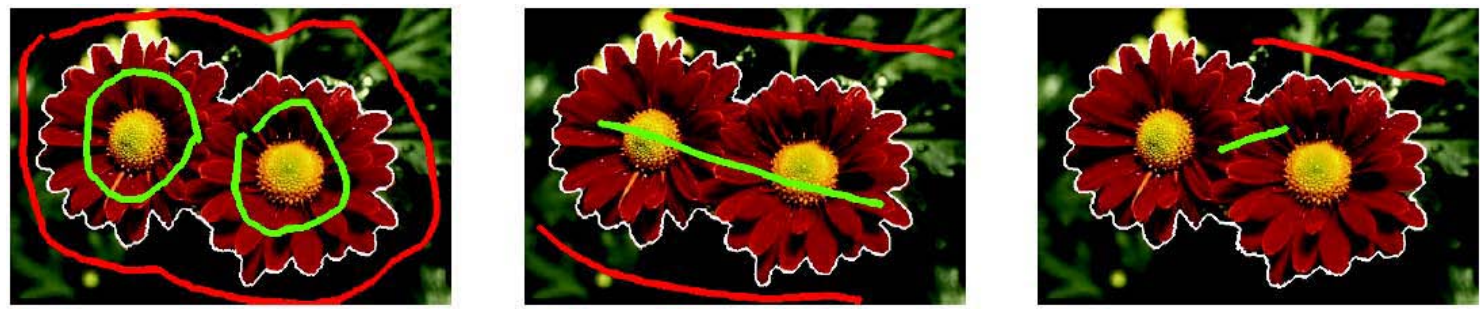

Figura 2.16: Diferentes estilos de traços, com resultados similares de segmentação.

Para avaliar a qualidade de nosso método, implementamos um protótipo em Java, disponível no sítio do SourceForge [1], testando os seguintes bancos de imagens naturais: Berkeley [59], Grabcut [76] e Bai e Sapiro [7]. Os resultados obtidos foram bastante satisfatórios, geralmente com traços extremamente simples. Seguindo a mesma idéia do trabalho apresentado em [7], a Figura 2.16 ilustra resultados similares de segmentação usando diferentes estilos simples de traços, indicando a robustez de nosso algoritmo.

A Figura 2.17 ilustra exemplos de qualidade, de segmentação e de composição de imagens com novo fundo. A Figura 2.18 ilustra nossos resultados usando imagens de 

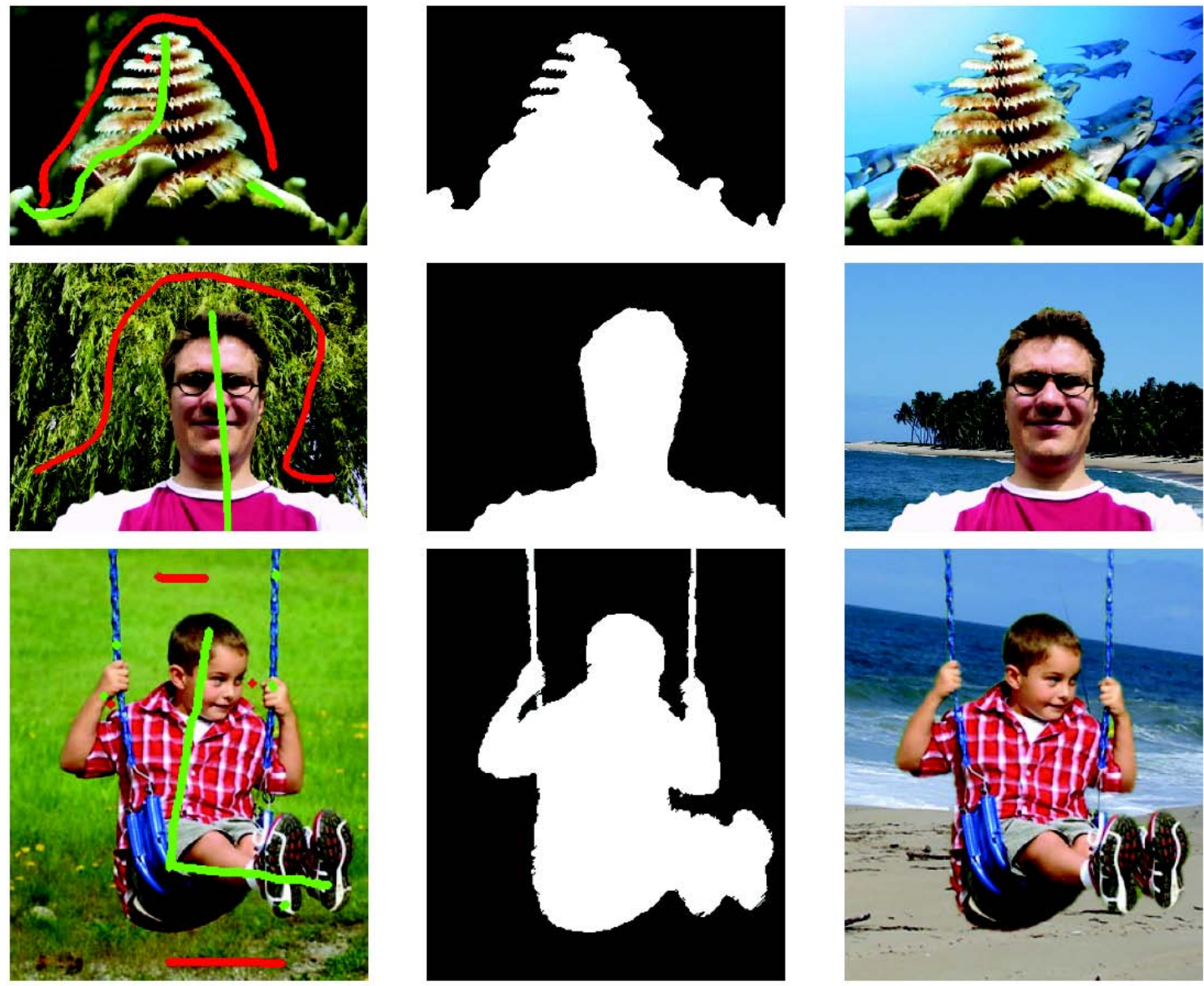

Figura 2.17: Exemplos de segmentação usando imagens de: Berkeley [59], Grabcut [76], Bai e Sapiro [7]. Coluna esquerda: imagens originais com traços do usuário. Coluna do meio: regiões rotuladas. Coluna direita: composições de imagens com um novo fundo.

Berkeley [59]. Similarmente, resultados sobre imagens de Grabcut [76] são exibidos na Figura 2.19. Resultados quantitativos, usando gabaritos, são exibidos nas Figuras 2.20 a 2.27, onde a qualidade de extração dos objetos é mensurada através do índice de Jaccard [42], comumente usado em experimentos envolvendo algoritmos de segmentação, conforme observado em [60], permitindo comparações de resultados entre diferentes trabalhos. Note que os bancos de imagens de Berkeley e Grabcut são bastante populares, possibilitando comparar diferentes métodos de segmentação.

Imagens adicionais e os respectivos resultados são ilustrados na Figura 2.28, apresentando dois exemplos com uma grande variabilidade de cores, resultando em diversas regiões de aparências similares, tanto no objeto quanto no fundo. Neste caso, usando 

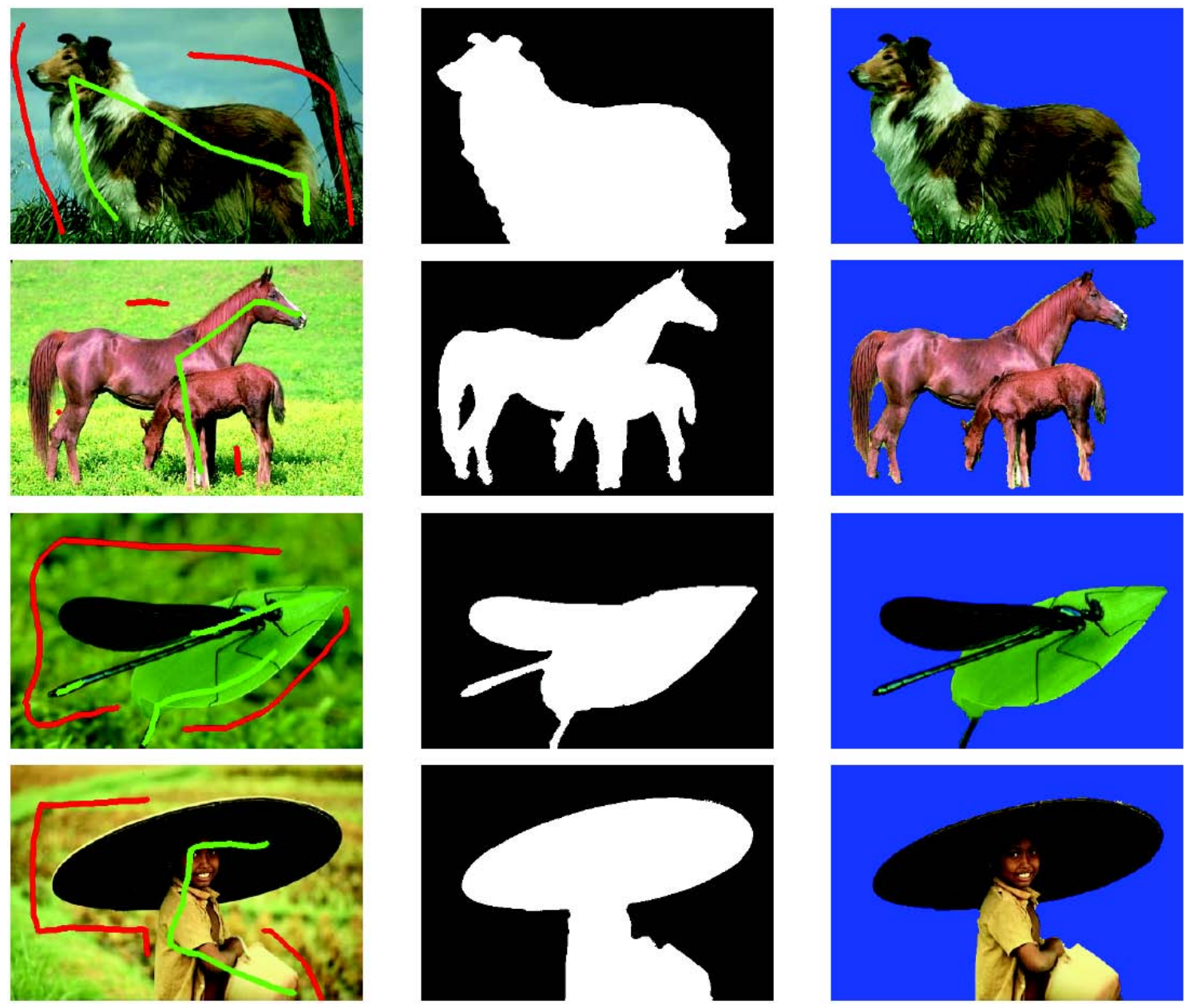

Figura 2.18: Banco de imagens de Berkeley [59]. Coluna esquerda: imagens originais com traços do usuário. Coluna do meio: regiões rotuladas. Coluna direita: objetos extraídos.

apenas as informações de aparência, representada pelas cores dos pixels, o processo de segmentação se torna uma tarefa bastante ambígua. Em geral, algoritmos como [41] não produzem o resultado desejado, demonstrando assim a importância das informações estruturais codificadas nas arestas dos grafos.

Uma clara vantagem do método DG é a possibilidade de aplicação do mesmo algoritmo, CalculaHomomorfismo $\left(G_{i}, G_{m}\right)$, Seção 2.3.3, para o caso de múltiplos rótulos (Figura 2.29), já que o cálculo do casamento não depende das cores dos vértices, definidas pelos traços do usuário. Uma outra vantagem é que os métodos baseados em regiões não dependem fortemente da largura dos traços do usuário, ao contrário dos métodos baseados em pixels, que geralmente se aproveitam de traços mais grossos para coletar 

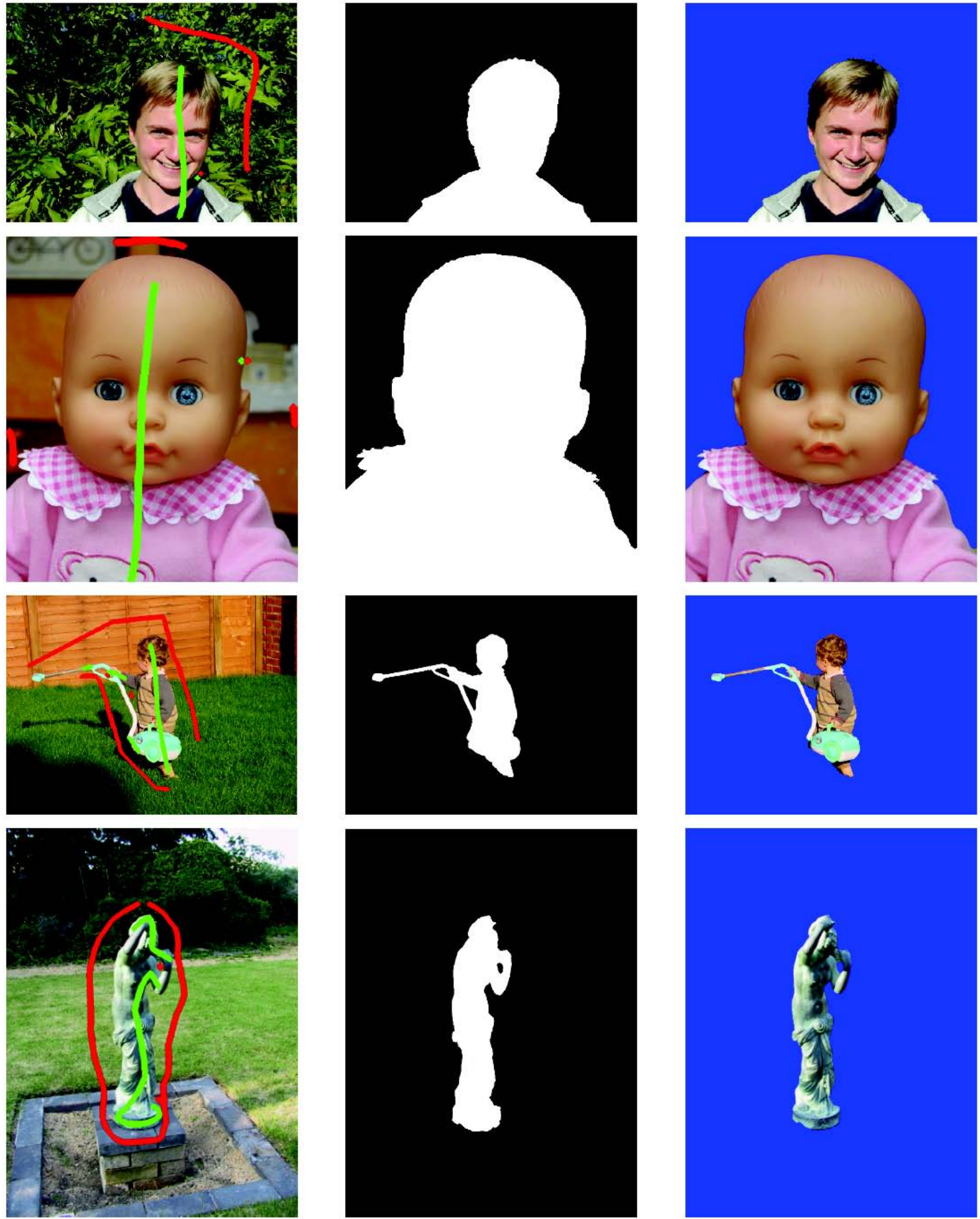

Figura 2.19: Banco de imagens Grabcut [76]. Coluna esquerda: imagens originais com traços do usuário. Coluna do meio: regiões rotuladas. Coluna direita: objetos extraídos. 

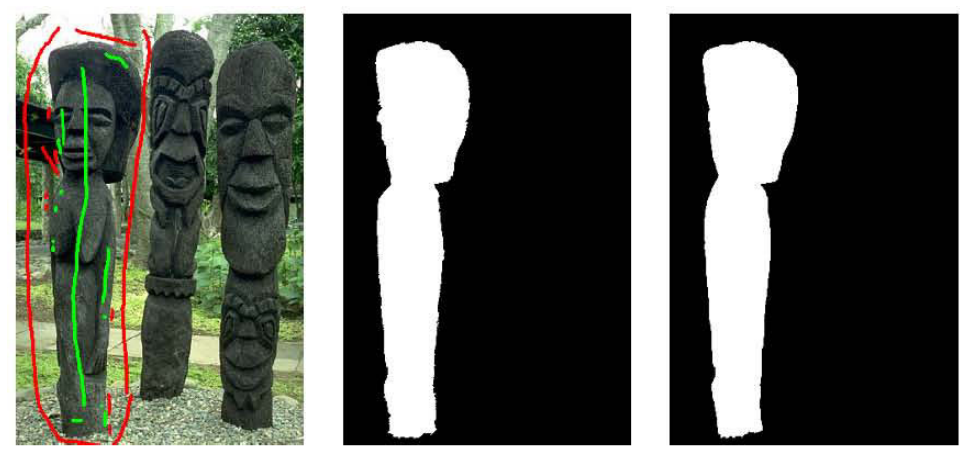

0.9768
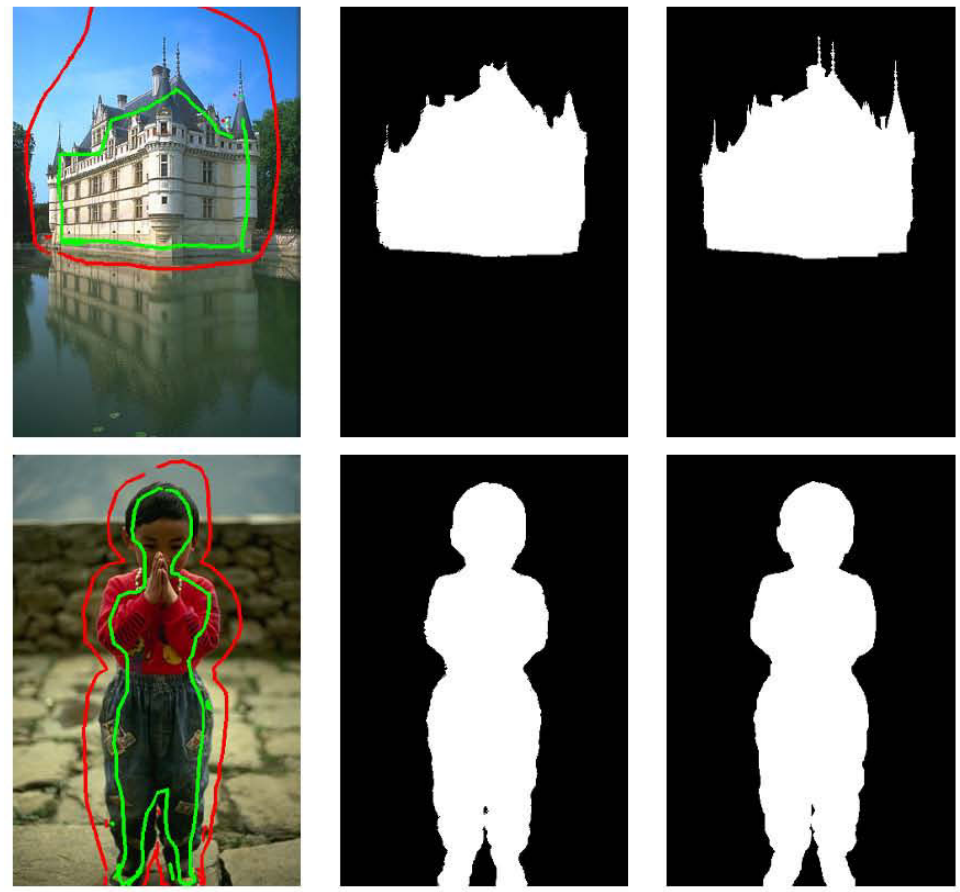

0.9725
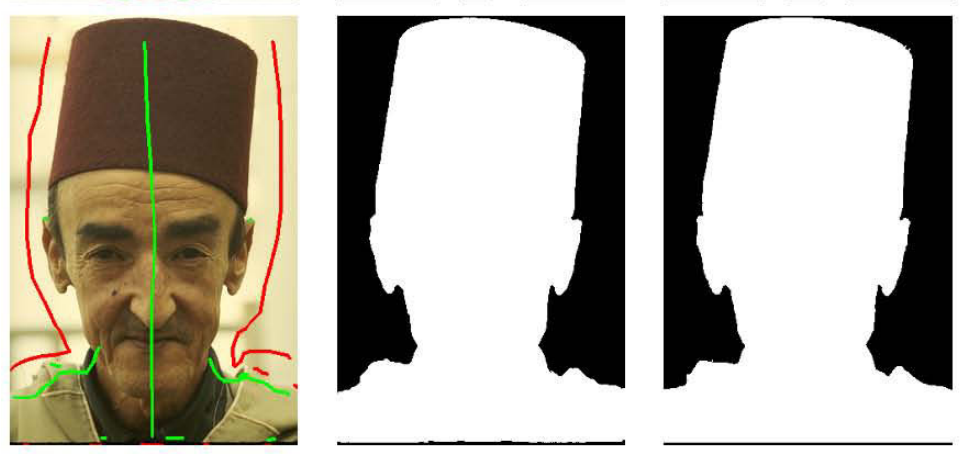

0.9782

(Tracos do usuario)

(Nosso resultado)

(Gabarito)

0.9907

(Indice de Jaccard)

Figura 2.20: Resultados quantitativos usando gabaritos de [60] e imagens de Berkeley [59]. 

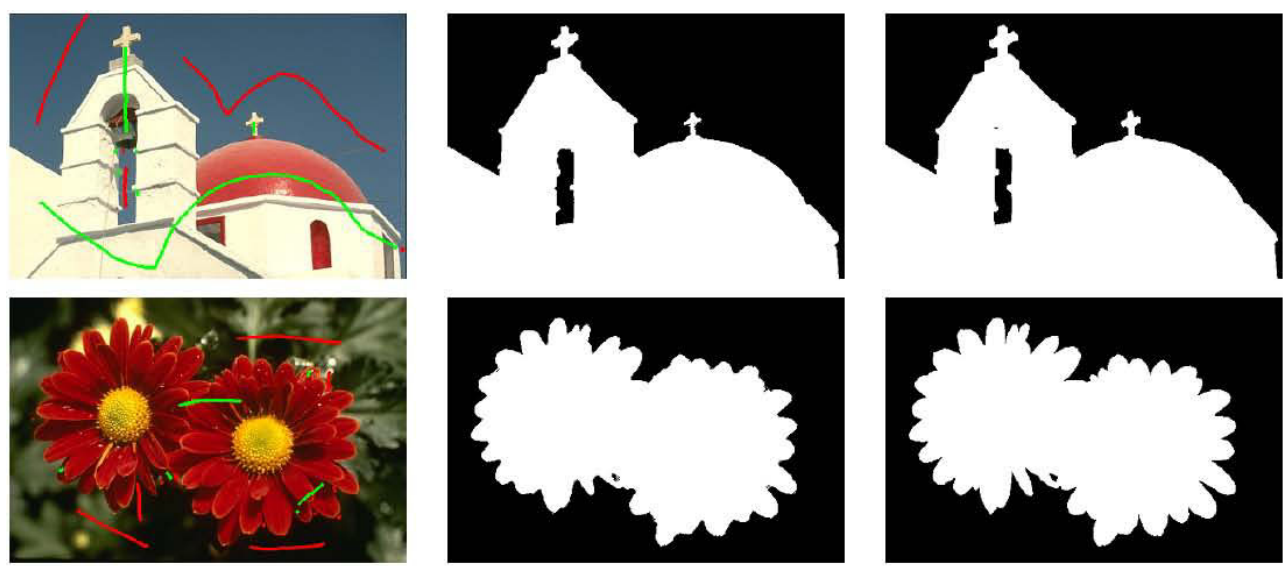

0.9889
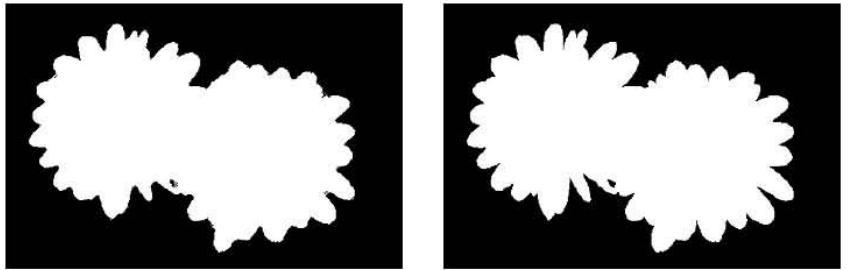

0.9781
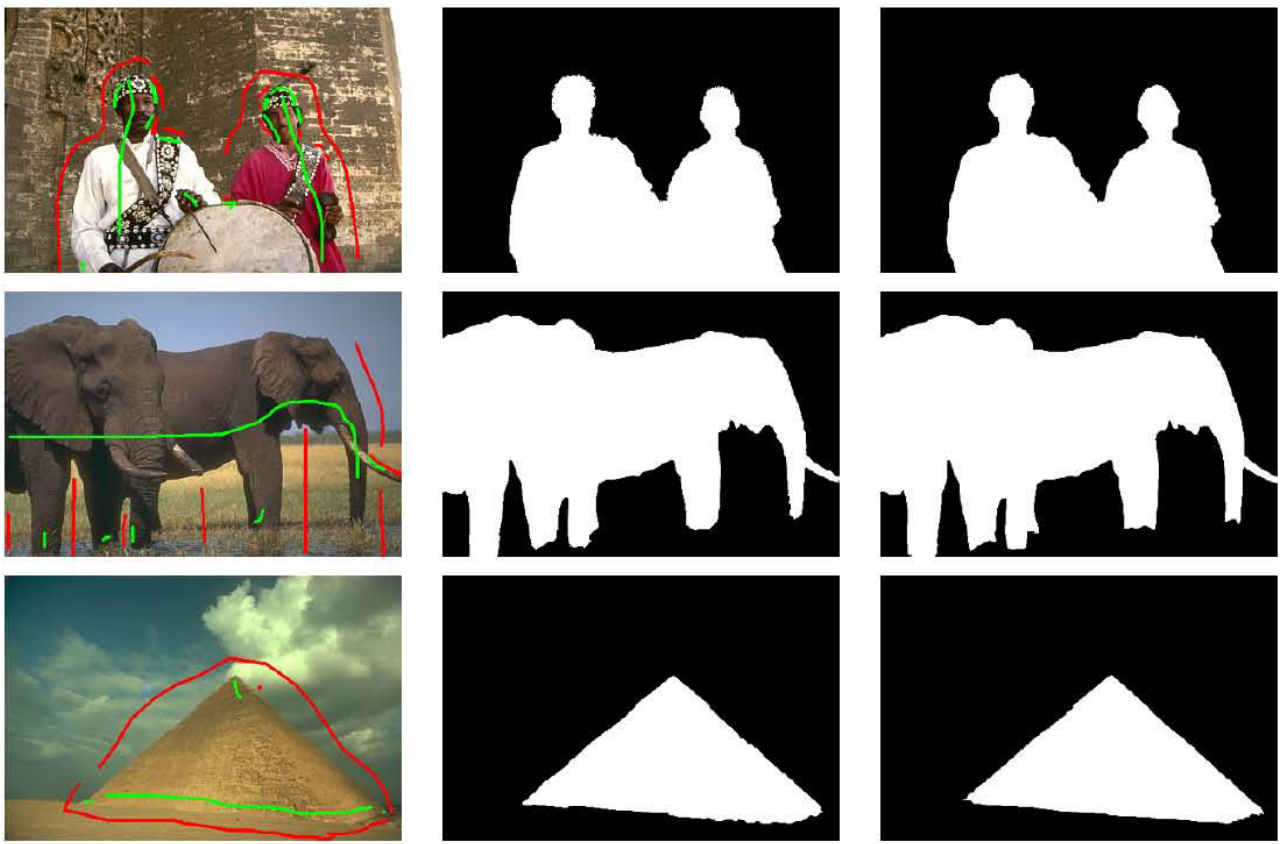

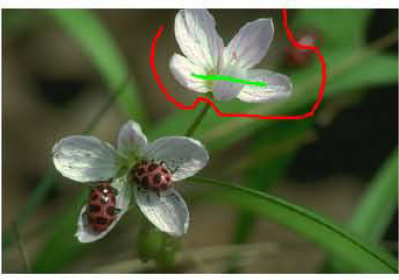

(Tracos do usuario)

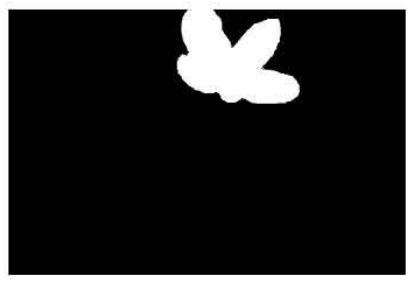

(Nosso resultado)

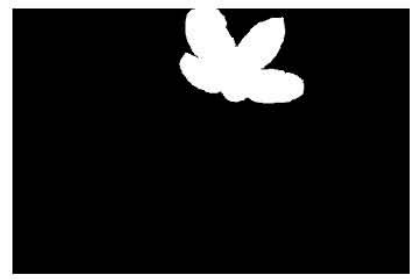

(Gabarito)
0.9872

0.9865

0.9788

0.9751 (Indice de Jaccard)

Figura 2.21: Resultados quantitativos usando gabaritos de [60] e imagens de Berkeley [59]. 

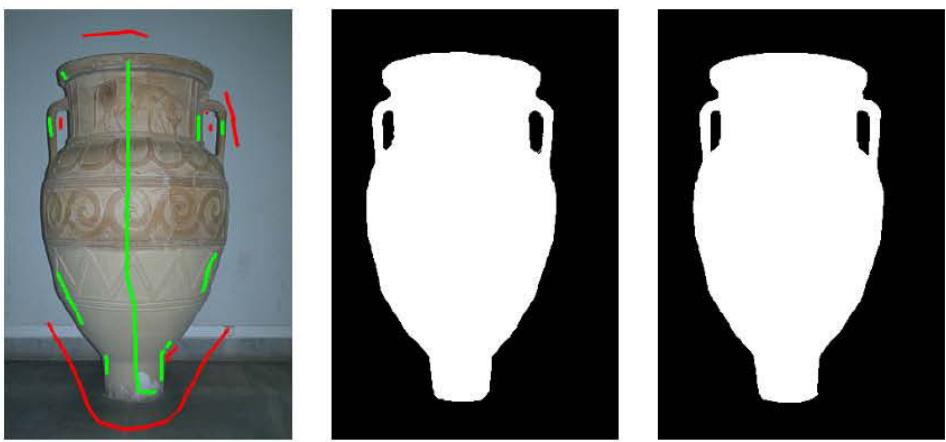

0.9897
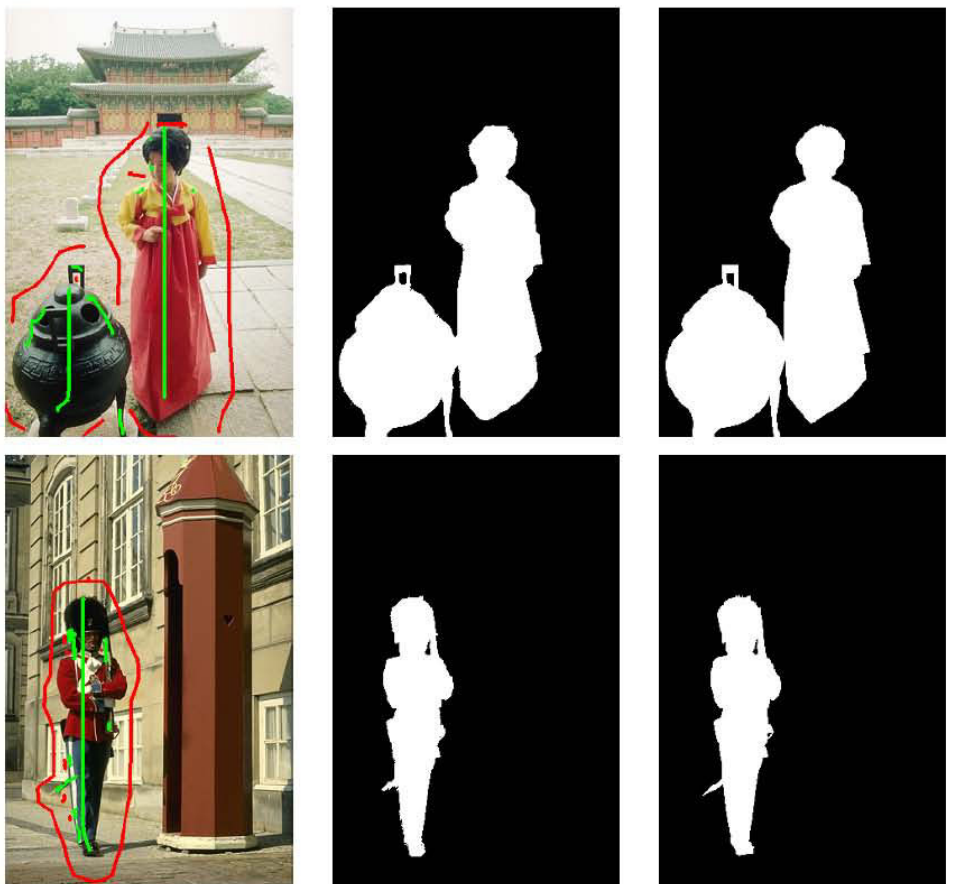

0.9815

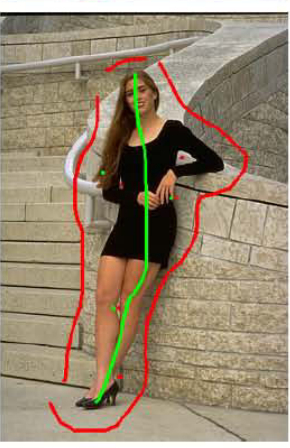

(Tracos do usuario)

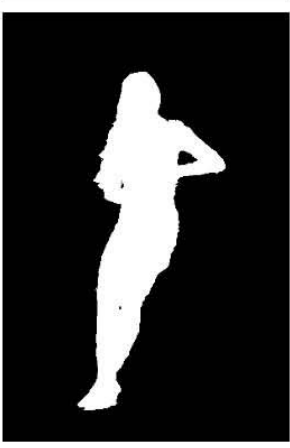

(Nosso resultado)

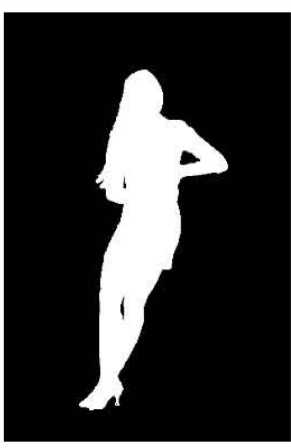

(Gabarito)
0.9710

0.9723

(Indice de Jaccard)

Figura 2.22: Resultados quantitativos usando gabaritos de [60] e imagens de Berkeley [59]. 

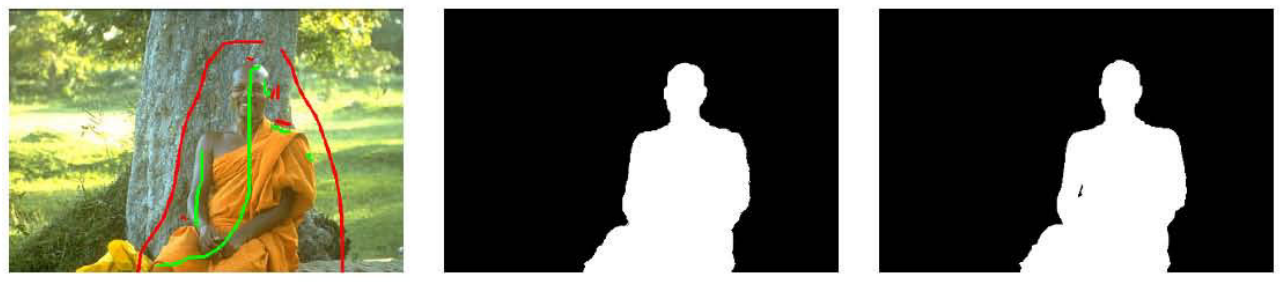

0.9857
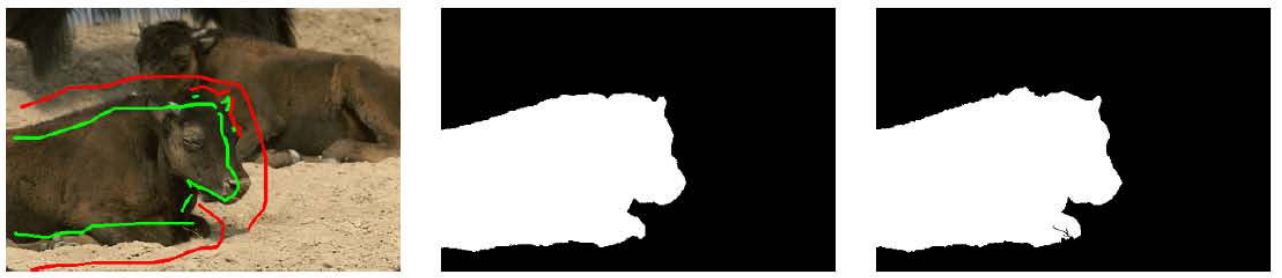

0.9854
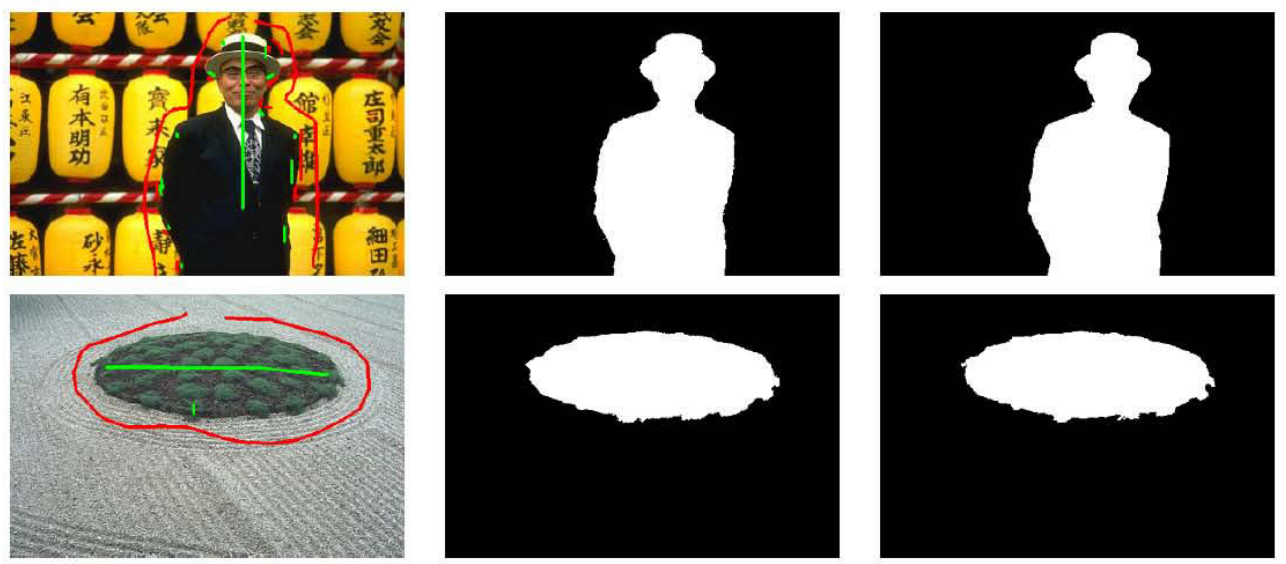

0.9909
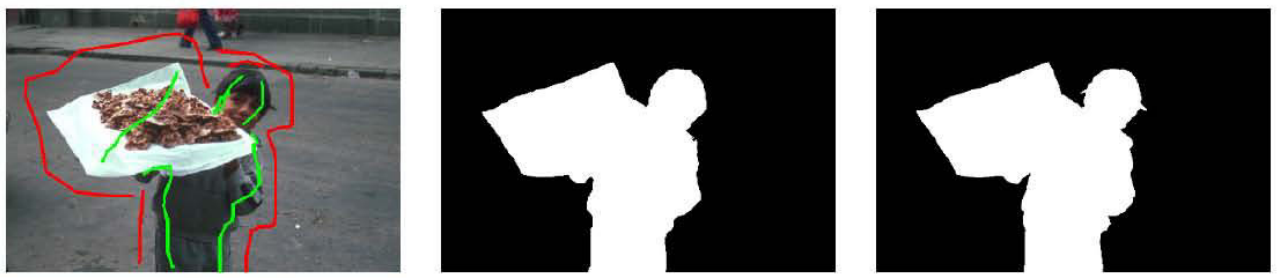

0.9837
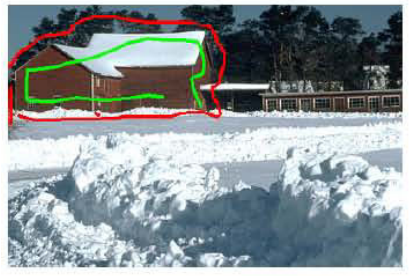

(Tracos do usuario)

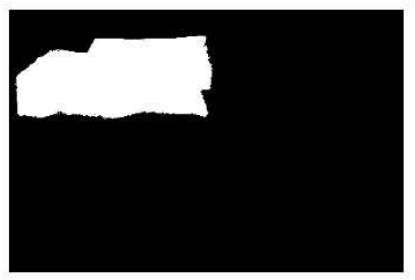

(Nosso resultado)

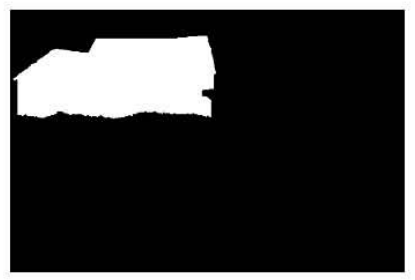

(Gabarito)
0.9757

(Indice de Jaccard)

Figura 2.23: Resultados quantitativos usando gabaritos de [60] e imagens de Berkeley [59]. 

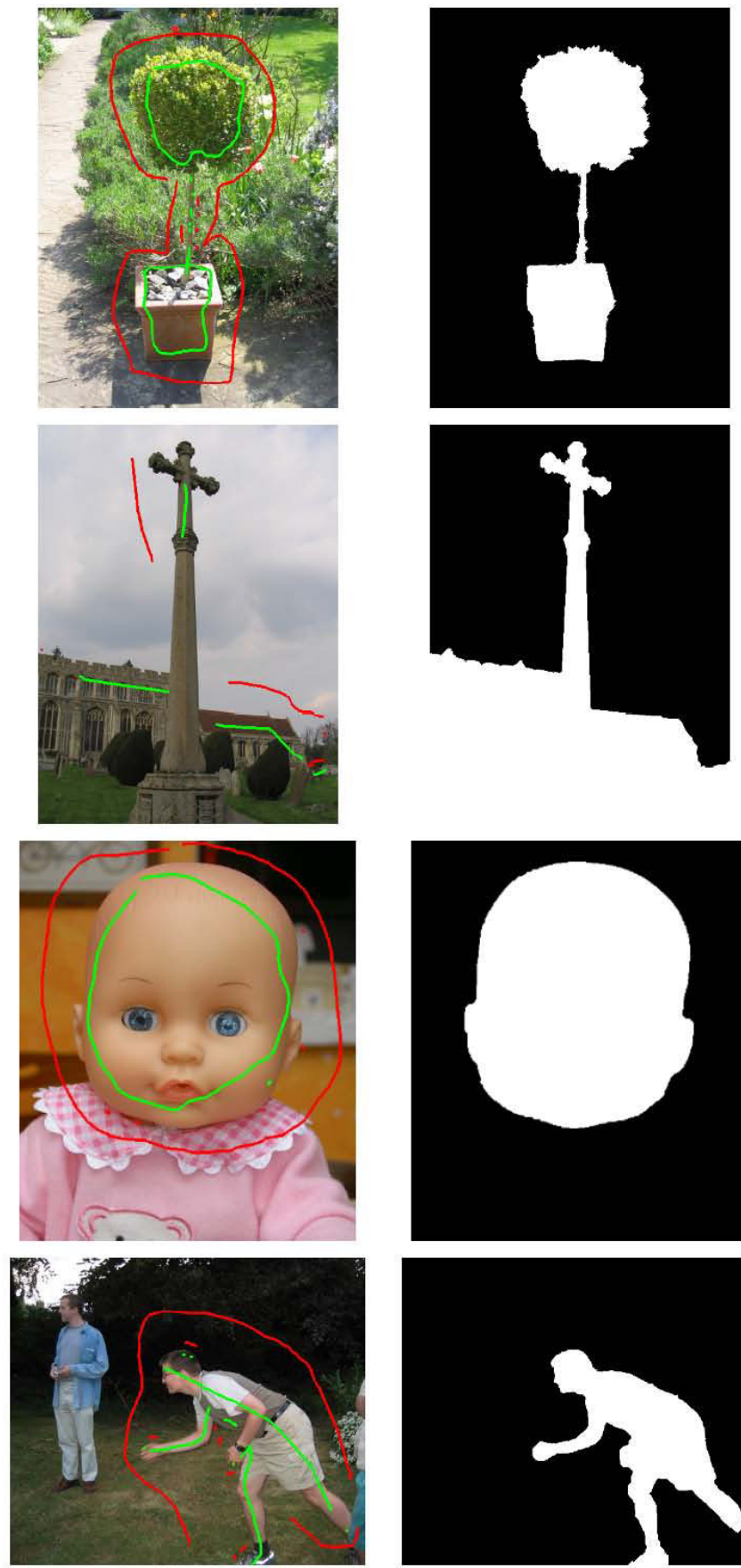

(Tracos do usuario)

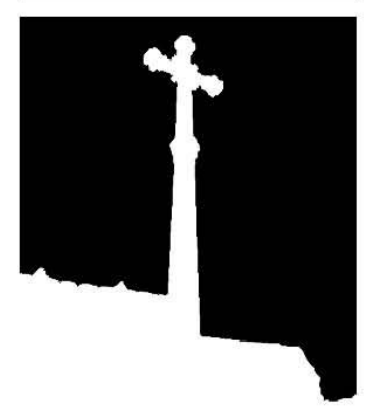

(Nosso resultado)

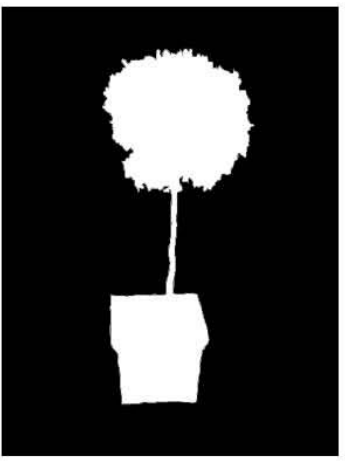

0.9678

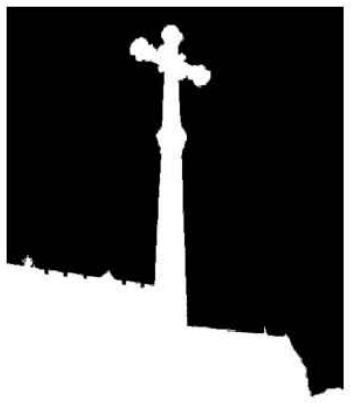

0.9978
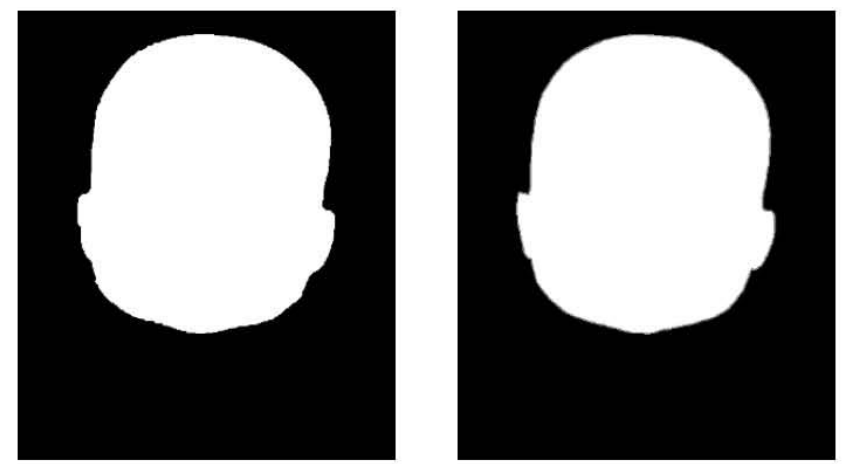

0.9987

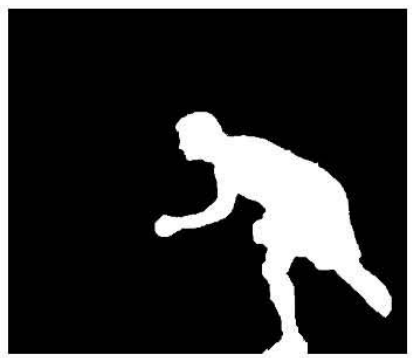

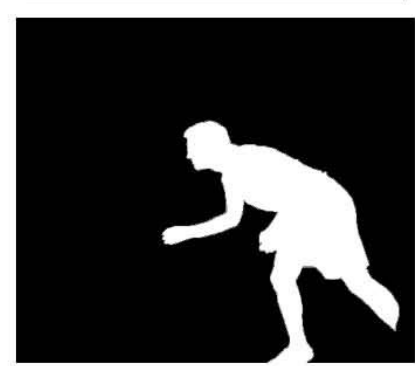

(Gabarito)
0.9808 (Indice de Jaccard)

Figura 2.24: Resultados quantitativos usando imagens de Grabcut [76]. 

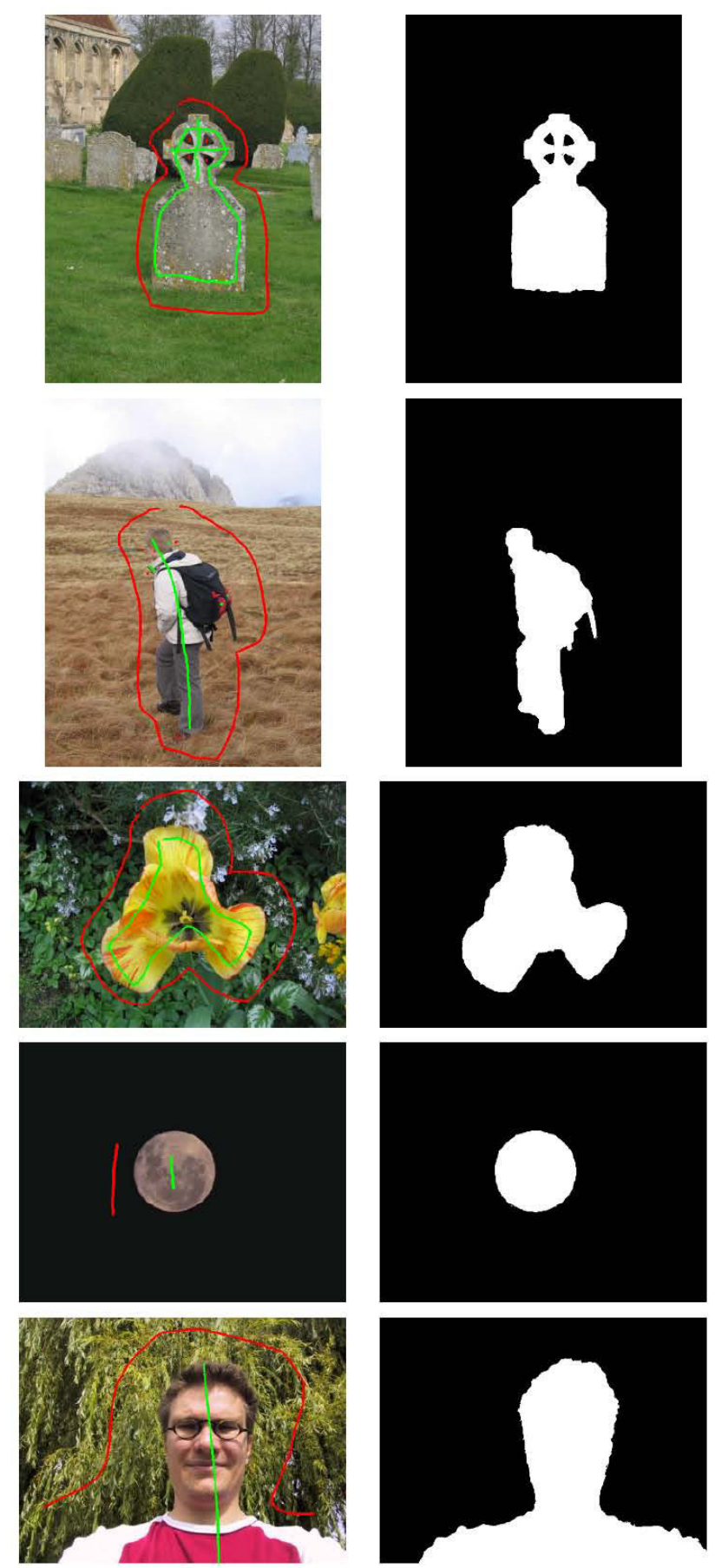

(Tracos do usuario)
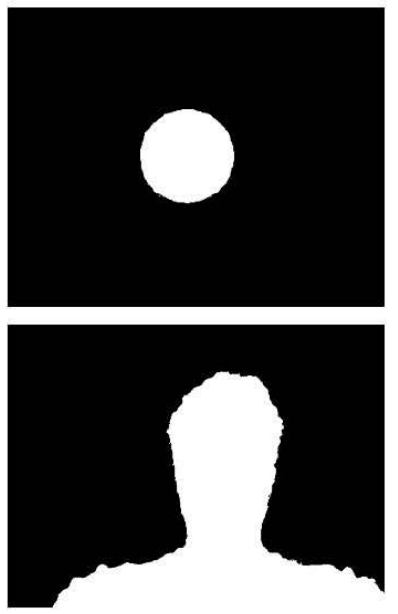

(Nosso resultado)

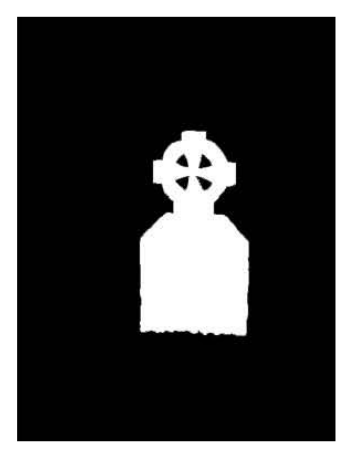

0.9820

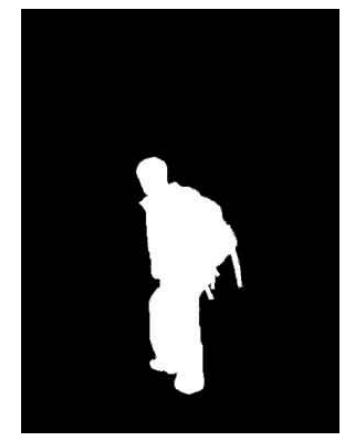

0.9850

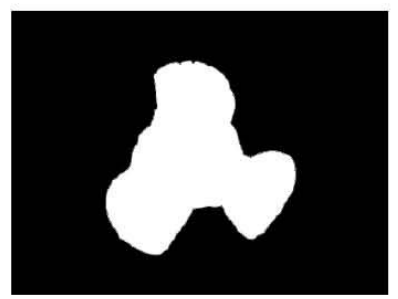

0.9972
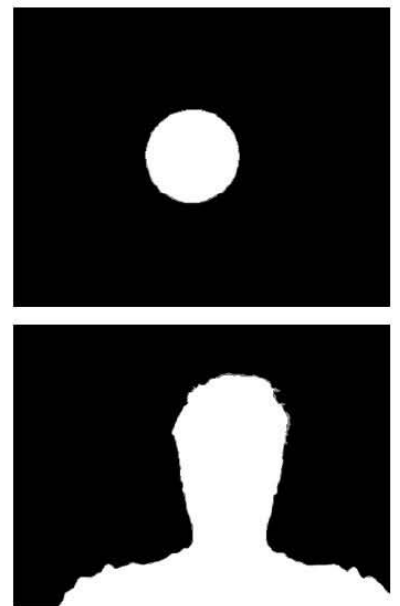

(Gabarito)

\subsection{5}

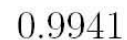

(Indice de Jaccard)

Figura 2.25: Resultados quantitativos usando imagens de Grabcut [76]. 

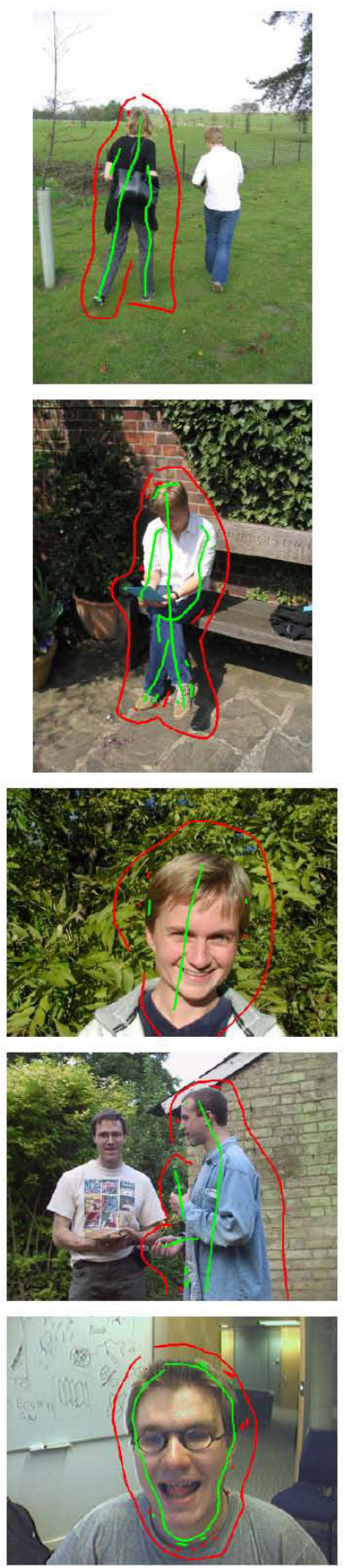

(Tracos do usuario)
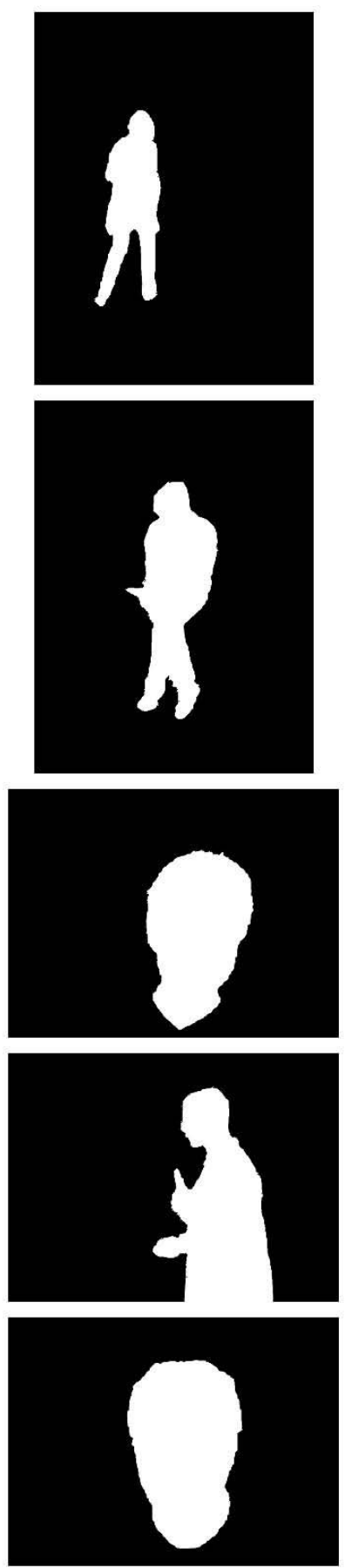

(Nosso resultado)

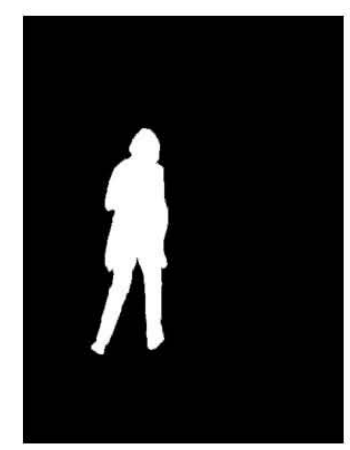

0.9882

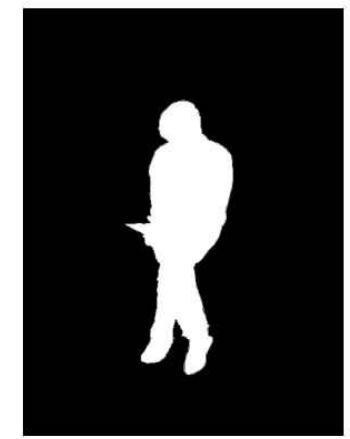

0.9858

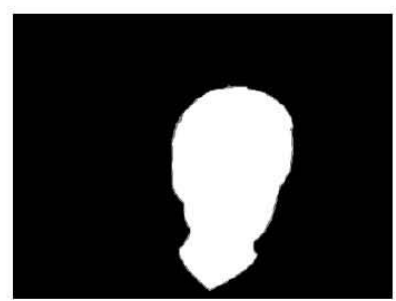

0.9947

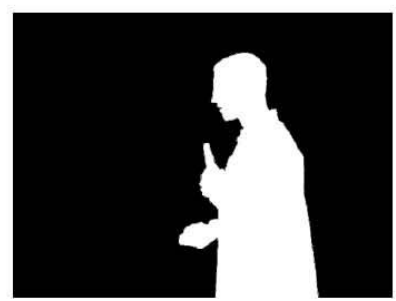

0.9938

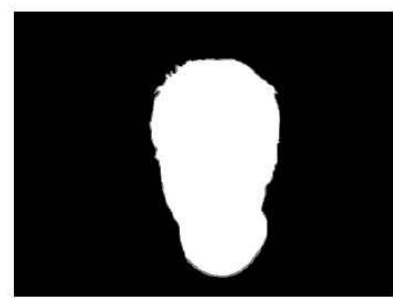

(Gabarito)
0.9848

(Indice de Jaccard)

Figura 2.26: Resultados quantitativos usando imagens de Grabcut [76]. 

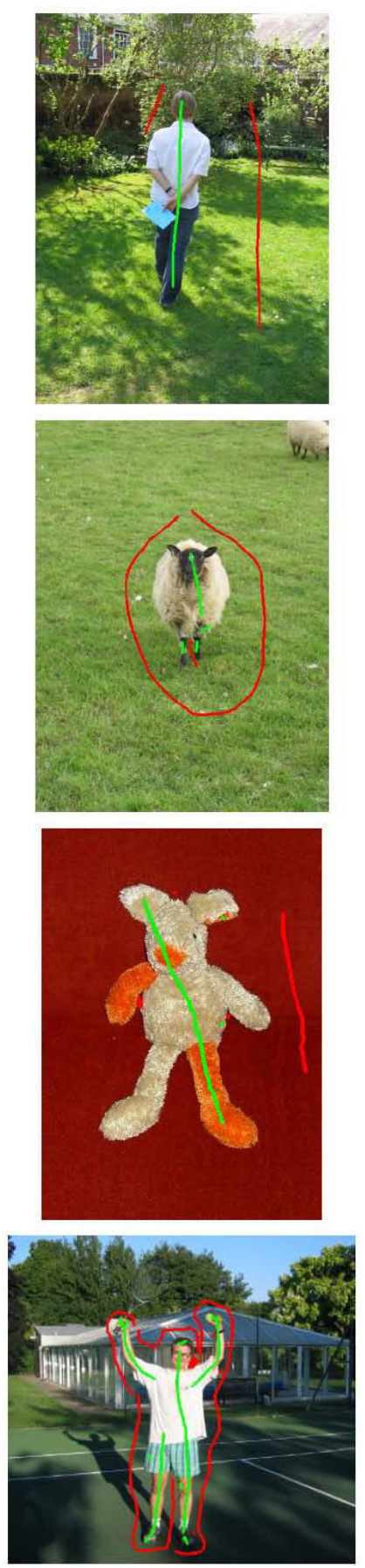

(Tracos do usuario)
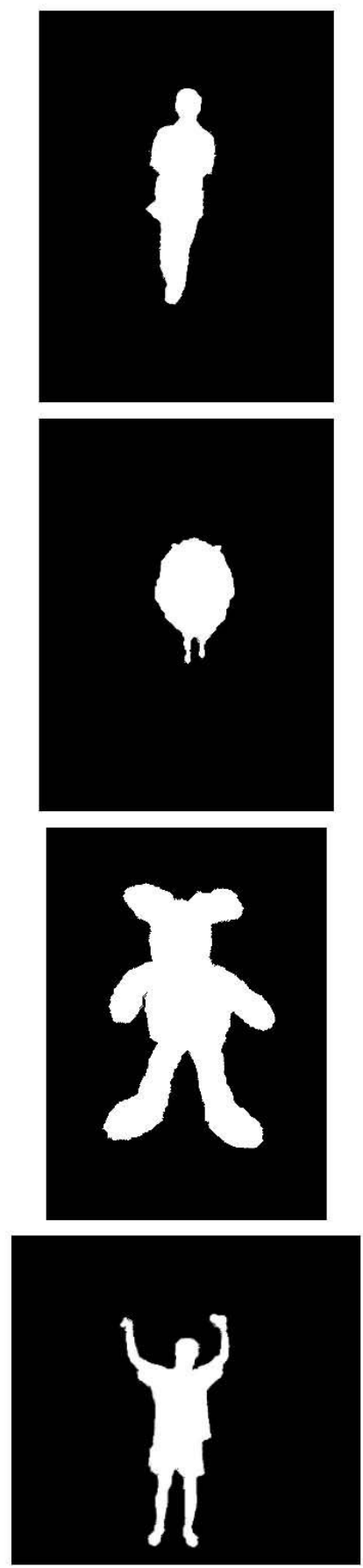

(Nosso resultado)

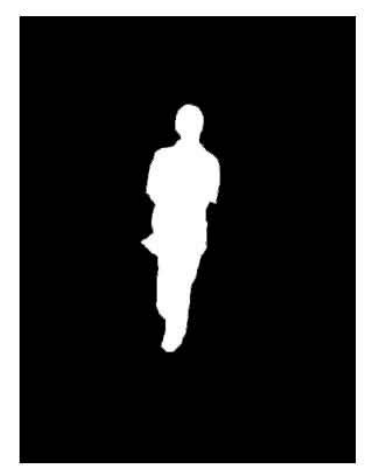

0.9907

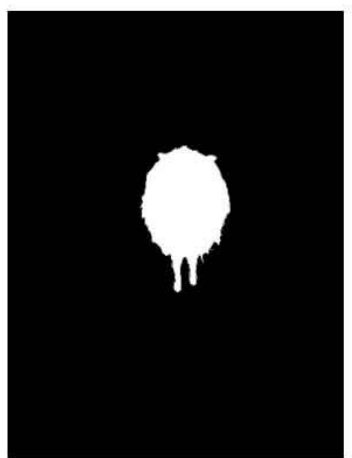

0.9794

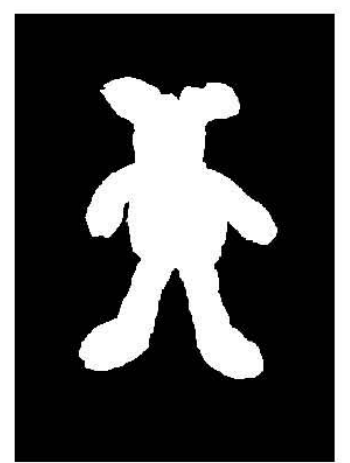

0.9635

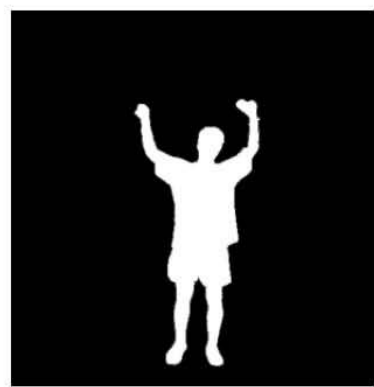

(Gabarito)
0.9739

(Indice de Jaccard)

Figura 2.27: Resultados quantitativos usando imagens de Grabcut [76]. 

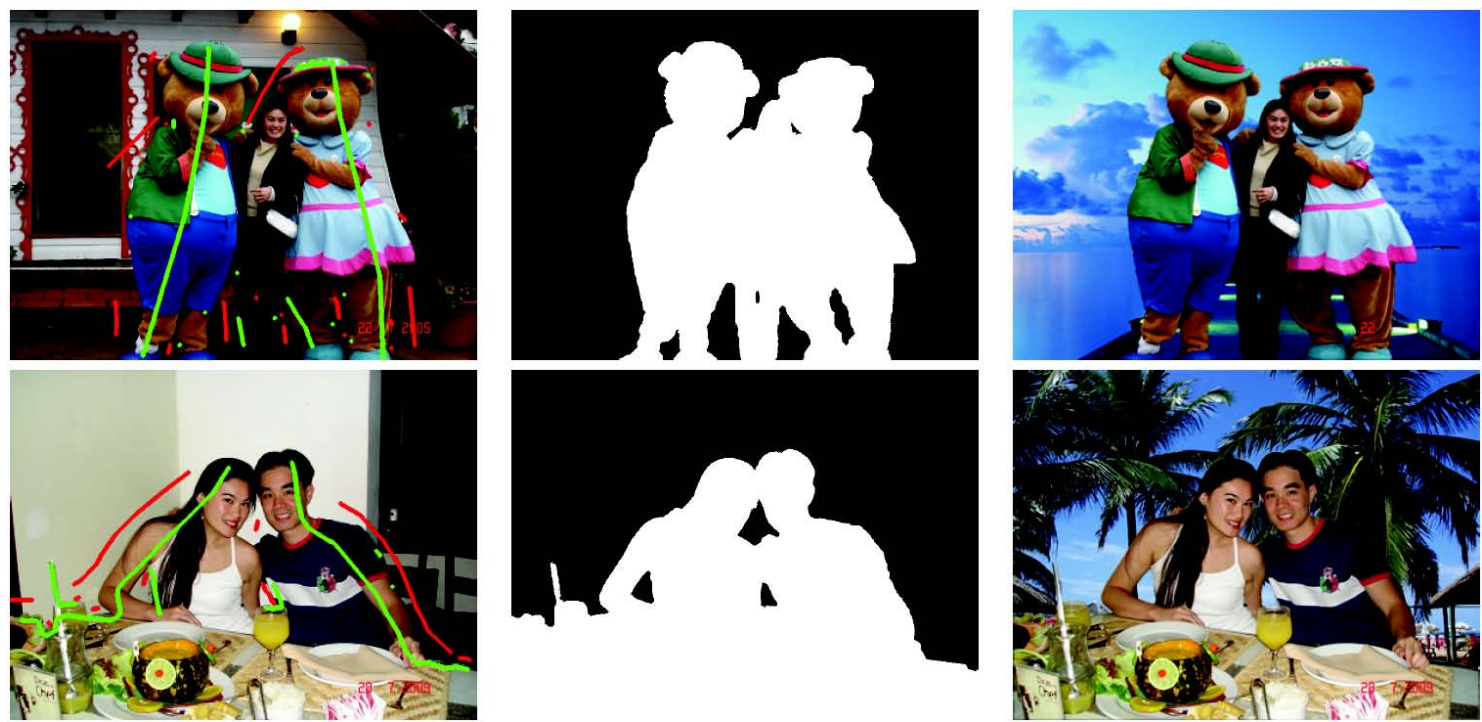

Figura 2.28: Exemplos adicionais de segmentação. Coluna esquerda: imagens originais com traços do usuário. Coluna do meio: regiões rotuladas. Coluna direita: composições de imagens com um novo fundo.

um número maior de pixels, acumulando mais estatísticas sobre as cores dos pixels com uma quantidade menor de traços. Em nossa implementação, usamos traços de dois pixels de largura, permitindo uma maneira 'elegante e limpa' do sistema interagir com o usuário. Nesta Tese, os resultados foram apresentados com traços dilatados para uma melhor visualização.
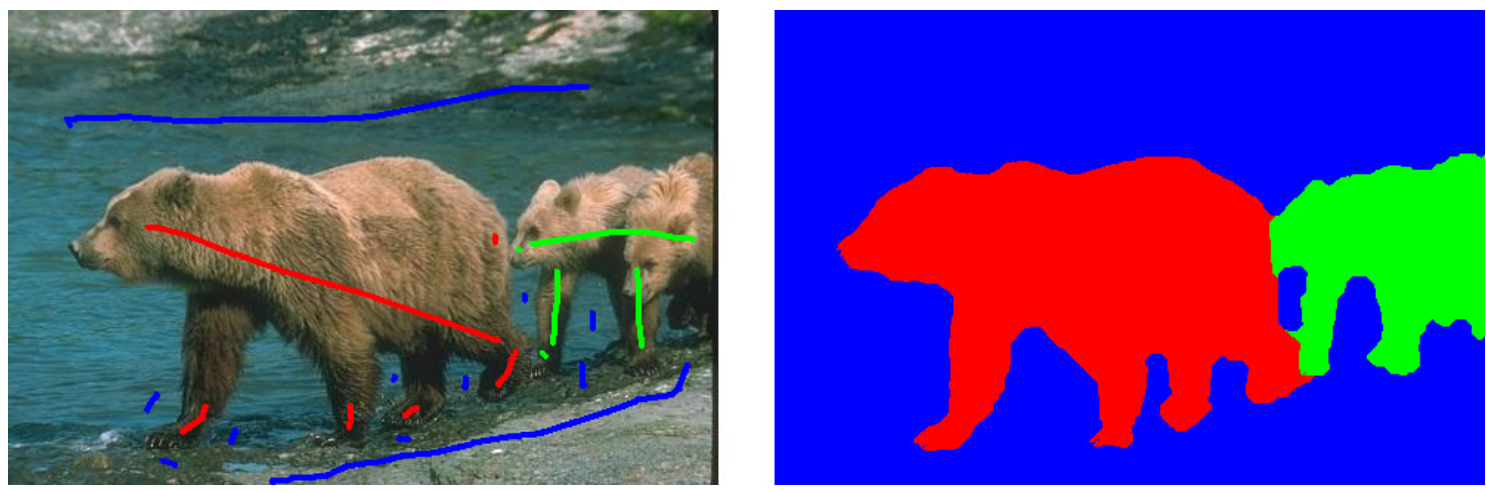

Figura 2.29: Exemplo de segmentação interativa com múltiplos rótulos. Coluna esquerda: Imagem original com traços do usuário. Coluna direita: regiões rotuladas.

Os experimentos foram executados em um computador com processador Intel Quad Core $2.4 \mathrm{GHz}$ e 4GB de memória RAM. A Tabela 2.2 apresenta os respectivos tempos de 
execução para cada uma das imagens usadas nas Figuras 2.18, 2.19 e 2.28. Para cada imagem, exibimos suas dimensões (largura e altura), a quantidade de vértices de cada grafo, o tempo consumido para a construção de cada grafo, finalizando com o tempo de execução do algoritmo CalculaHomomorfismo $\left(G_{i}, G_{m}\right)$, pós-processamento (Seção 2.5.4) e o total. Note que a maior quantidade de tempo gasto foi na construção do grafo de entrada $G_{i}$. Isto se deve ao pré-processamento usado para reduzir o número de regiões do watershed, fundindo pequenas regiões, menores que 25 pixels em nossos experimentos, com a região adjacente de aparência mais próxima, usando a Equação 2.8. Isto causou uma certa lentidão na hora de inicializar o sistema, mas sem afetar a interatividade do sistema com o usuário. Além disso, foi extremamente importante pois reduziu drasticamente o número de regiões, conforme exibido na Tabela 2.3, contribuindo consideravelmente para a redução dos tamanhos dos dois grafos e, consequentemente o tempo de cálculo do casamento. Na Tabela 2.2, apesar do fato de que programas escritos em Java são geralmente mais lentos que os programas escritos, por exemplo, em $\mathrm{C}++$, note que a atualização do modelo e o cálculo do casamento foram bastante rápidos, justificando a aplicação de nosso método para o problema de segmentação interativa de imagens.

Uma ilustração qualitativa é dada na Figura 2.30, onde usamos a implementação de McGuinness e Connor [60], comparando o nosso método com três algoritmos implementados: seeded region growing (SRG) [3], simple interactive object extraction (SIOX) [41], e interactive graph cuts (IGC) [17]. Na Figura 2.30, o objetivo foi extrair a pirâmide do fundo. Neste caso, as cores da pirâmide e da areia são bastante similares. Métodos baseados na informação de aparência falham neste cenário, como é o caso do SRG e do SIOX. Já o método IGC produziu um resultado melhor, pois este método inclui um termo regularizador, balanceando a restrição de suavidade e o contraste das bordas dos objetos. Mas neste exemplo, o IGC teve alguns problemas nas bordas da pirâmide. Ao contrário, o nosso método produziu um resultado mais satisfatório, pois as bordas mais importantes estavam presentes no watershed.

Uma comparação quantitativa é ilustrada na Tabela 2.4, onde usamos a base de dados de Blake e associados [16]. Este banco possui 50 imagens, sendo particularmente interessante devido ao fato de incluir máscaras, usadas no papel dos traços do usuário. Por exemplo, na Figura 2.31, o objetivo é extrair as duas flores do fundo. Para isto, a região branca foi usada para marcar as duas flores e a região cinza escuro foi usada para 
Tabela 2.2: Tempos de execução para a computação da segmentação inicial pelo algoritmo de casamento, e para o refinamento pelo pós-processamento, usando as Figuras 2.18, 2.19 e 2.28 .

\begin{tabular}{lccr|rrrrr} 
& & & & \multicolumn{5}{|c}{ Tempos computacionais (em milisegundos) } \\
Imagem & Tamanho & $\left|V_{i}\right|$ & $\left|V_{m}\right|$ & $G_{i}$ & $G_{m}$ & Casamento & Pós-proc & Total \\
\hline berk-dog & $480 \times 320$ & 1431 & 185 & 3516 & 422 & 819 & 1233 & 5990 \\
berk-horse & $480 \times 320$ & 1015 & 63 & 3791 & 203 & 454 & 2193 & 6641 \\
berk-insect & $480 \times 320$ & 2144 & 244 & 3782 & 234 & 1735 & 1234 & 6985 \\
berk-boy & $480 \times 320$ & 1976 & 186 & 3218 & 261 & 1182 & 1293 & 5954 \\
grab-person2 & $600 \times 450$ & 2350 & 117 & 4789 & 263 & 923 & 1711 & 7686 \\
grab-doll & $462 \times 549$ & 2836 & 128 & 4718 & 208 & 1093 & 1458 & 7477 \\
grab-statue & $768 \times 1024$ & 5175 & 315 & 18019 & 667 & 4289 & 6377 & 29352 \\
grab-child & $1024 \times 768$ & 6214 & 314 & 17709 & 556 & 4702 & 4770 & 27737 \\
add-bears & $800 \times 600$ & 5557 & 386 & 10480 & 326 & 5696 & 4644 & 21146 \\
add-couple & $800 \times 600$ & 4025 & 325 & 10127 & 562 & 3327 & 3249 & 17265 \\
\hline
\end{tabular}

marcar o fundo. Já as regiões cinza claro e preta foram usadas para indicar as regiões em dúvida, a serem classificadas. Na Tabela 2.4, comparamos o nosso método DG com alguns dos principais estados-da-arte. Por exemplo, usando $\lambda_{1}=0.5$ para cada uma das 50 imagens, obtivemos um resultado comparável ao random walker [46]. Em geral, diferentes imagens necessitam de diferentes valores para $\lambda_{1}$, balanceando a importância entre o termo de aparência e o termo estrutural na função custo para obter o 'melhor' resultado de segmentação. Usando um valor para o parâmetro $\lambda_{1}$, associado ao 'melhor' resultado para cada uma das imagens, reduzimos a taxa média de erro, superando um resultado recentemente publicado em [71]. Para cada imagem, a taxa de erro foi calculada conforme descrita em [16], dividindo-se o total de pixels classificados incorretamente sobre o total de pixels nas regiões em dúvida. 
Tabela 2.3: Regiões do watershed: quantidade original e reduzida, respectivamente, usando as Figuras 2.18, 2.19 e 2.28 .

\begin{tabular}{lcr} 
& \multicolumn{2}{c}{ Número de regiões do watershed } \\
Imagem & Original & Pré-proc \\
\hline berk-dog & 14420 & 1431 \\
berk-horse & 17471 & 1015 \\
berk-insect & 9620 & 2144 \\
berk-boy & 11205 & 1976 \\
grab-person2 & 27705 & 2350 \\
grab-doll & 15446 & 2836 \\
grab-statue & 90294 & 5175 \\
grab-child & 84025 & 6214 \\
add-bears & 34591 & 5557 \\
add-couple & 27595 & 4025 \\
\hline
\end{tabular}

Tabela 2.4: Comparação quantitativa, usando as imagens de Blake e associados [16].

\begin{tabular}{lc} 
Method & Error rate \\
\hline GMMRF [16] & $7.9 \%$ \\
IGC [17] & $6.7 \%$ \\
Random walker [46] & $5.4 \%$ \\
DG ( $\left.\lambda_{1}=0.5\right)$ & $\mathbf{5 . 4 \%}$ \\
Geodesic GC [71] & $4.8 \%$ \\
DG ('melhor' $\left.\lambda_{1}\right)$ & $\mathbf{4 . 2 \%}$ \\
\hline
\end{tabular}




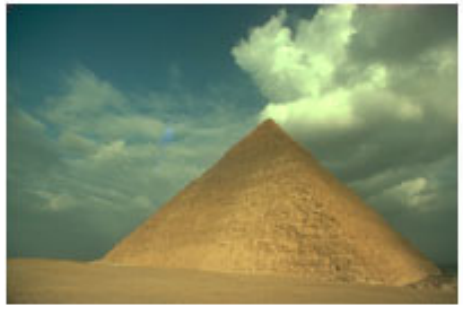

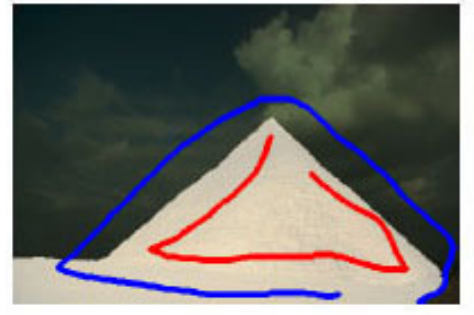

(SRG)

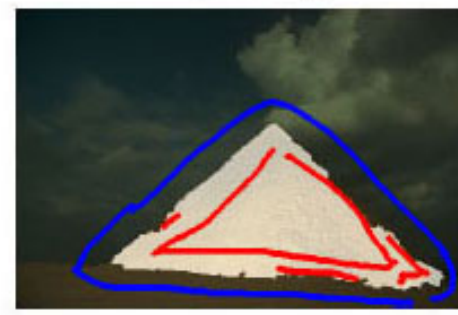

(IGC)

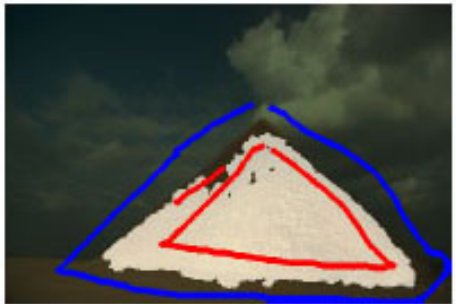

(SIOX)

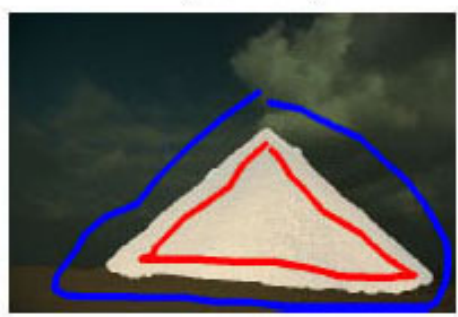

(DG)

Figura 2.30: Comparação qualitativa entre seeded region growing (SRG) [3], simple interactive object extraction (SIOX) [41], interactive graph cuts (IGC) [17], e o nosso método DG.
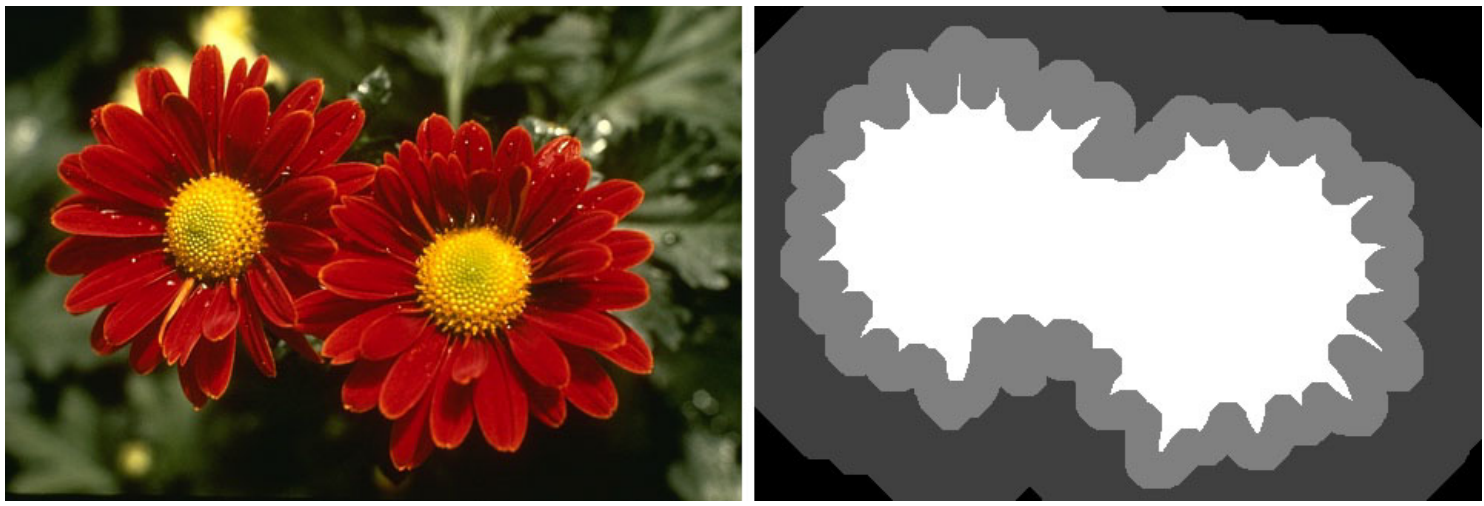

Figura 2.31: Imagem original e a respectiva máscara usada no papel dos traços do usuário. 


\subsection{Resumo da abordagem DG}

Em nossa primeira abordagem, um grafo auxiliar chamado de grafo deformado (DG) foi proposto para avaliar, de maneira eficiente, as informações estruturais dadas pelas relações espaciais entre pontos.

Para o casamento entre gels de eletroforese bidimensional (2DEM), nosso algoritmo se baseou em dois componentes principais: uma técnica gulosa para estimar as correspondências e uma estratégia baseada em iterative closest point (ICP) [13], estimando as coordenadas de alinhamento do grafo modelo sobre o padrão de pontos da entrada para computar DGs adequados. Durante a fase de estimação da correspondência, a chave para obter eficiência foi o fato do algoritmo sempre comparar grafos de mesma topologia, usando DGs para avaliar as informações estruturais.

O método proposto foi comparado com algoritmos conhecidos na literatura, envolvendo distância Euclidiana, shape context, casamento de grafos bipartidos, estimação de transformações envolvendo thin-plate splines e ICP. Os experimentos envolveram o banco de dados público [2], onde produzimos resultados satisfatórios relativos ao par "095-098", representando um caso desafiador, com um grande número de pontos e grandes deformações não-rígidas entre os pontos correspondentes. Usamos testes artificiais sobre este par complexo para justificar que o nosso método é bastante promissor para explorar o conhecimento a priori, onde o deslocamento inicial poderia ser inicializado a partir de algumas poucas correspondências validadas pelo usuário. De maneira geral, os resultados confirmaram a necessidade das informações estruturais para obter melhores resultados, além da eficiência dos DGs para avaliar estas informações.

Também baseada em DGs, uma versão simplificada da abordagem foi aplicada ao problema de segmentação interativa de imagens naturais (INIS). Neste caso, os dois grafos a serem casados já estão alinhados, pois são obtidos de uma mesma imagem, não havendo a necessidade de se usar a estratégia ICP. Apesar de sua simplicidade, a abordagem gulosa baseada em DGs, combinada a um pós-processamento, produziu resultados de qualidade para uma variedade de imagens naturais. Os experimentos indicaram que o algoritmo de casamento é rápido na prática, podendo ser usado de maneira interativa. Além disso, o algoritmo não é restrito a segmentação binária e pode ser aplicado para múltiplos rótulos, representando múltiplas partes de objetos [45, 64]. 


\section{Capítulo 3}

\section{Casamento de padrões de pontos via campos aleatórios de Markov}

No capítulo anterior, o casamento de padrões de pontos foi formulado como um casamento entre grafos. Antes de apresentar a segunda contribuição, o casamento entre grafos é formulado por campos aleatórios de Markov (MRFs), fornecendo os requisitos necessários para as seções subsequentes.

A segunda abordagem é baseada em uma formulação Bayesiana de máxima a posteriori, seguindo a mesma formulação de atribuição quadrática definida no capítulo anterior. Combinada com representações esparsas de objetos, este método é aplicado com sucesso ao casamento entre formas (shape matching, SM) [63]. Resultados bastante promissores foram obtidos para o problema de colorização assistida por computador (CAC) [67]. O método é baseado no eficiente algoritmo de propagação de crenças (BP) via mínimaconvolução [39].

\subsection{Campos aleatórios de Markov}

A teoria sobre campos aleatórios de Markov (MRF) proporciona uma maneira conveniente de representar informações contextuais de objetos ou entidades, representados por padrões de pontos ou outro tipo de características espacialmente correlacionadas, caracterizando as influências mútuas entre as entidades através de probabilidades. A praticidade desta 
teoria é largamente atribuída à equivalência entre MRFs e as distribuições de Gibbs, estabelecida por Hamersley e Clifford (1971).

MRFs têm sido aplicados com sucesso a problemas de visão, como cálculo de disparidades de imagens estéreo e restauração de imagens. Atualmente, existem duas técnicas bastante populares usadas para estimar MRFs: graph cuts (GC) [18] e belief propagation (BP) [39]. Métodos GC são baseados em uma implementação eficiente do algoritmo de corte-mínimo/fluxo-máximo em grafos. As abordagens BP são baseadas em propagação de mensagens. Enquanto os métodos GC dependem de condições particulares para minimizar a função de energia $[18,50]$, os algoritmos BP não sofrem nenhuma explícita restrição em relação a função custo, sendo mais genéricos neste sentido. Nossa segunda contribuição é um método baseado em BP.

A seguir, apresentamos os ingredientes principais de um MRF, necessários para descrever nosso método baseado em BP. Seguindo [92], mostramos que minimizar a Equação 2.1, definida pela atribuição quadrática (Seção 2.2), equivale a estimar a máxima a posteriori de um certo MRF. Uma revisão mais detalhada sobre MRFs pode ser encontrada em [54].

\subsubsection{Ingredientes principais de um MRF}

Seja $F=\left\{F_{1}, \ldots, F_{k}\right\}$ uma família de variáveis aleatórias definidas em um conjunto de sítios $\mathcal{S}$. Suponha que cada variável aleatória $F_{i}$ assuma um valor $f_{i}$ em um conjunto de rótulos $\mathcal{L}$. $F$ é chamado de campo aleatório.

$F_{i}=f_{i}$ denota o evento em que $F_{i}$ assume o valor $f_{i}$. $\left(F_{1}=f_{1}, \ldots, F_{k}=f_{k}\right)$ denota o evento conjunto. Por simplicidade, um evento conjunto é abreviado por $F=f$, onde $f=\left\{f_{1}, \ldots, f_{k}\right\}$ é uma configuração de $F$, correspondente a uma realização do campo.

Para um conjunto discreto de rótulos $\mathcal{L}$, a probabilidade de uma variável aleatória $F_{i}$ assumir um valor $f_{i}$ é denotado por $P\left(F_{i}=f_{i}\right)$, e abreviado por $P\left(f_{i}\right)$. A probabilidade conjunta é denotada por $P(F=f)=P\left(F_{1}=f_{1}, \ldots, F_{k}=f_{k}\right)$, e abreviada por $P(f)$.

$F$ é um campo aleatório de Markov em $\mathcal{S}$, com respeito a um sistema de vizinhança $\mathcal{N}$, se e somente se as seguintes propriedades são satisfeitas:

$$
\begin{aligned}
\text { (Positiva) } & P(f)>0, \forall f \in F \\
\text { (Markoviana) } & P\left(f_{i} \mid f_{\mathcal{S} \backslash\{i\}}\right)=P\left(f_{i} \mid f_{\mathcal{N}(i)}\right)
\end{aligned}
$$


onde $\mathcal{S} \backslash\{i\}$ representa a diferença entre conjuntos, $f_{\mathcal{S} \backslash\{i\}}$ é o conjuto dos rótulos nos sítios em $\mathcal{S} \backslash\{i\}$ e $f_{\mathcal{N}(i)}$ é o conjunto dos rótulos nos sítios vizinhos de $i$.

A propriedade (Positiva) é assumida por questões técnicas e geralmente pode ser satisfeita na prática. Por exemplo, quando esta propriedade é satisfeita, a probabilidade conjunta de qualquer campo aleatório é únicamente determinada pelas probabilidades (locais) condicionais [12].

A propriedade (Markoviana) define as características locais do campo aleatório $F$ : um rótulo interage somente com os seus rótulos vizinhos. Por exemplo, nosso método BP aproveita representações eficientes baseadas em vizinhanças esparsas, conforme descrito na Seção 3.4.2.

\subsubsection{Casamento entre grafos via MRFs}

A seguir, seguimos uma notação compatível com a utilizada na literatura MRF.

Dados dois grafos, um de entrada $G_{i}$ e um modelo $G_{m}$, definimos um MRF no grafo de entrada $G_{i}, \mathcal{S}=V_{i}$. Desta maneira, para cada vértice de entrada $p \in V_{i}$, existe uma variável aleatória $F_{p}$, assumindo valores $f_{p}=\alpha \in V_{m}$ no conjunto de rótulos $\mathcal{L}=V_{m}$. O sistema de vizinhança é dado pelo conjunto das arestas do grafo de entrada, $\mathcal{N}=E_{i}$.

A propriedade (Markoviana) definida na Equação 3.2 restringe a dependência de um dado vértice a apenas aos seus vizinhos, sendo reescrita como:

$$
P\left(f_{p} \mid f_{V_{i} \backslash\{p\}}\right)=P\left(f_{p} \mid f_{E_{i}(p)}\right)
$$

onde $E_{i}(p)$ denota o conjunto dos vizinhos de $p$ no grafo de entrada.

Um trabalho importante envolvendo MRFs e casamento entre grafos é devido a Caelli e Caetano [20]. Os autores definem um MRF no grafo modelo $G_{m}$, assumindo a existência de um único 'sinal' (modelo) imerso na 'cena' (entrada, representando o sinal com ruídos), especificamente desenhado para o problema de isomorfismo de subgrafo, aplicado a experimentos artificiais para casar padrões de segmentos de reta.

Neste trabalho, propomos uma generalização, definindo um MRF no grafo de entrada $G_{i}$, de modo que cada vértice de entrada seja rotulado, permitindo generalizar o casamento, tanto para homomorfismo quanto para MCS. O resultado da rotulação representa um homomorfismo. Para obter um MCS, aplicamos o pós-processamento descrito 
nas Seções 2.3.3 e 3.3 para 'desempatar' rótulos, convertendo um homomorfismo para um MCS.

Anguelov et al. [5] propuseram uma formulação baseada também em um mapeamento partindo da cena para o modelo. Mas neste caso, os autores inverteram os papéis considerados aqui e em [20], assumindo que a cena é uma visão parcial ou completa do modelo. Tanto em [20] como no nosso método, assume-se que o modelo é uma visão parcial ou completa da cena, permitindo uma procura por ocorrências do modelo dentro da cena (veja o exemplo de clutter e os exemplos de segmentação de múltiplos objetos nas Figuras 3.8 e 4.1, respectivamente).

\subsubsection{MRFs em termos de campos aleatórios de Gibbs}

O teorema de Hamersley e Clifford [12] estabelece uma maneira adequada de especificar um MRF. Este teorema prova a equivalência entre MRFs e campos aleatórios de Gibbs (GRFs). Antes de escrever um MRF em termos de GRFs, definimos GRFs. Um GRF é determinado pela distribuição de Gibbs:

$$
P(f)=Z^{-1} \cdot \exp \left(-\sum_{c \in \mathcal{C}} V_{c}(f)\right)
$$

onde $\mathcal{C}$ é o conjunto de todos os cliques. Um conjunto de vértices é chamado de clique se cada vértice do conjunto é vizinho dos demais vértices, pertencentes ao conjunto. $Z$ é uma constante de normalização. $\left\{V_{c}(f)\right\}$ é o conjunto das funções potenciais, definidas para avaliar cliques, mapeando configurações a números reais.

Logo, para definir um MRF, basta especificar as funções potenciais de clique. No nosso caso, isto é feito da seguinte maneira. Considerando a formulação quadrática definida na Seção 2.2, assumimos que, para todos os cliques de tamanhos distintos de dois, a função potencial de clique vale zero. Para cliques de tamanho dois, a função potencial de clique é definida por:

$$
V_{c}(f)=M_{p, q}^{\prime}\left(f_{p}, f_{q}\right)=M^{\prime}\left(f_{p}, f_{q}\right)
$$

onde $M^{\prime}\left(f_{p}, f_{q}\right)$ é chamado de componente de Markov, com o mesmo papel que o termo quadrático da Equação 2.1. Reescrevendo a Equação 3.4, a distribuição conjunta de um 
MRF pode ser definida por:

$$
P(f)=Z^{-1} \cdot \exp \left(-\sum_{(p, q) \in \mathcal{N}} M^{\prime}\left(f_{p}, f_{q}\right)\right)
$$

\subsection{Formulação via máxima a posteriori}

A teoria MRF permite formulações com princípios estabelecidos de otimalidade, baseados em teorias de decisão e de estimação estatística. Máxima a posteriori (MAP) é um dos critérios estatísticos de otimalidade mais populares e mais utilizados em visão computacional. Esta técnica, no contexto de MRFs, resulta no famoso arcabouço MAP-MRF, popularizado por Geman e Geman [43] e outros, amplamente adotado pela maioria dos trabalhos envolvendo MRFs.

Geralmente, o campo $F$ não é diretamente observável e é preciso estimar sua configuração $f$, baseada em uma observação D, no nosso caso, representando as informações de aparência, relacionada a $f$ através de uma função de verossimilhança $P(D \mid f)$.

Por uma função a priori $P(f)$, é possível representar as informações contextuais de objetos, no nosso caso, representados por pontos distribuídos no espaço, favorecendo certos padrões através de atribuições de valores mais altos de probabilidades.

Pela regra de Bayes, a probabilidade a posteriori pode ser definida como:

$$
p(f \mid \mathrm{D})=\frac{p(\mathrm{D} \mid f) \cdot p(f)}{p(\mathrm{D})}
$$

Para estimar 'a melhor' configuração $f$, usamos a técnica MAP:

$$
f^{*}=\arg \max _{f \in F} p(\mathrm{D} \mid f) \cdot p(f)
$$

onde o objetivo é encontrar uma configuração que maximiza a distribuição a posteriori, definida na Equação 3.7.

Agora precisamos definir $p(\mathrm{D} \mid f)$ de maneira apropriada para obter uma formulação de atribuição quadrática, similar a da Seção 2.2. Seja $\mathrm{D}_{p}$ a aparência observada no vértice $p$. Considerando que ruídos sobre a aparência de cada vértice ocorrem de maneira independente, temos que:

$$
p(\mathrm{D} \mid f)=\prod_{p \in V_{i}} p\left(\mathrm{D}_{p} \mid f_{p}\right)
$$


Para as informações de aparência, assumimos que:

$$
p\left(\mathrm{D}_{p} \mid l\right)=C_{p} \cdot \exp \left(-D_{p}(l)\right) \quad \text { para } l \in \mathcal{L},
$$

onde $C_{p}$ é uma constante de normalização e $D_{p}$ possui o mesmo papel do termo linear da Equação 2.1, avaliando as informações de aparência. A verossimilhança pode ser então definida por:

$$
p(\mathrm{D} \mid f) \propto \exp \left(-\sum_{p \in V_{i}} D_{p}\left(f_{p}\right)\right)
$$

Reescrevendo a Equação 3.8, substituindo $p(\mathrm{D} \mid f)$ e $p(f)$ pelas Equações 3.11 e 3.6, respectivamente, temos que:

$$
f^{*}=\arg \max _{f \in F} \exp \left(-\sum_{p \in V_{i}} D_{p}\left(f_{p}\right)-\sum_{(p, q) \in \mathcal{N}} M^{\prime}\left(f_{p}, f_{q}\right)\right)
$$

que é equivalente a minimizar a seguinte função de energia, usada para mensurar a qualidade de um mapeamento ou rotulação $f: V_{i} \rightarrow V_{m}$ :

$$
E(f)=\sum_{p \in V_{i}} D_{p}\left(f_{p}\right)+\lambda_{1} \sum_{(p, q) \in E_{i}} M\left(f_{p}, f_{q}\right)
$$

onde $\lambda_{1}$ é um número real (positivo), balanceando a influência do termo quadrático sobre o resultado do casamento. Note que a Equação 3.13 é similar à Equação 2.1, mas usando a notação MRF.

\subsubsection{Termo linear $\left(D_{p}\right)$}

Similarmente ao capítulo anterior, cada vértice da entrada $p \in V_{i}$ possui um atributo $\mu_{i}(p)$ e cada vértice do modelo $\alpha \in V_{m}$ possui um atributo $\mu_{m}(\alpha)$. O termo linear $D_{p}\left(f_{p}\right)$ compara diretamente os atributos $\mu_{i}(p)$ e $\mu_{m}\left(f_{p}\right)$, atribuindo um custo proporcional à dissimilaridade dos dois vértices. Para cada aplicação específica, usamos diferentes atributos de vértices. Por exemplo, para o casamento entre formas (shape matching, SM), os atributos representam as informações de shape context [9], enquanto para o problema de colorização assistida por computador (CAC), usamos área e perímetro. 


\subsubsection{Termo quadrático: Markov $(M)$}

Para as duas aplicações, SM e CAC, adotamos as mesmas informações estruturais descritas na Seção 2.2.2. Cada aresta orientada $(p, q) \in E_{i}$ possui um atributo $\nu_{i}(p, q)$ no grafo de entrada. Similarmente é o caso para o modelo, $(\alpha, \beta) \in E_{m}, \nu_{m}(\alpha, \beta)$. O componente de Markov $M\left(f_{p}, f_{q}\right)$ compara $\nu_{i}(p, q)$ e $\nu_{m}\left(f_{p}, f_{q}\right)$, atribuindo um custo proporcional à dissimilaridade dos vetores, representando os atributos das arestas, avaliados pela função de penalidade geométrica definida na Equação 2.2. A definição completa do termo de Markov, para o algoritmo baseado em BP, é feita na Seção 3.3.1.

\subsection{Casamento via propagação de crenças (BP)}

Conforme descrito na Seção 3.2, encontrar um mapeamento que minimize a Equação 3.13 corresponde ao problema de máxima a posteriori (MAP). Para estimar uma solução, usamos um algoritmo de propagação de crenças (BP), formulado por distribuições de probabilidade, seguindo uma técnica equivalente ao máximo-produto, usando o negativo do log das probabilidades [39]. Neste caso, o máximo-produto é transformado em uma mínima-soma, apresentando menor sensibilidade a artefatos numéricos, correspondendo diretamente à energia definida na Equação 3.13 .

O método é baseado em troca de mensagens, propagadas pelo grafo de entrada $G_{i}$, de acordo com o sistema de vizinhança definido pelas arestas $E_{i}$. Cada mensagem é um vetor, cuja dimensão é dada pela quantidade de rótulos $\left|V_{m}\right|$, possibilitando, para cada vértice de entrada $q \in V_{i}$, obter os 'custos' de cada rótulo $f_{q} \in V_{m}$.

Seja $m_{p q}^{t}$ a mensagem que o vértice $p$ envia ao vizinho $q$, na iteração $t$. Inicialmente, todas as entradas do vetor $m_{p q}^{0}$ valem zero. No início de cada iteração, as novas mensagens são computadas por:

$$
m_{p q}^{t}\left(f_{q}\right)=\min _{f_{p}}\left(M\left(f_{p}, f_{q}\right)+D_{p}\left(f_{p}\right)+\sum_{s \in E_{i}(p) \backslash\{q\}} m_{s p}^{t-1}\left(f_{p}\right)\right)
$$

onde $E_{i}(p) \backslash\{q\}$ denota os vizinhos de $p$ exceto $q$. Após $T$ iterações ${ }^{1}$, para cada vértice

\footnotetext{
${ }^{1}$ Para obter boas soluções, $T$ deve ser suficiente para que as informações de aparência e de estrutura sejam propagadas por todo o grafo. Por exemplo, para árvores ou grafos com um único loop, $T$ deve ser suficiente para a solução convergir ou oscilar de maneira periódica.
} 
de entrada, calculamos um vetor de confianças (belief vector), representando os custos de cada rótulo:

$$
b_{q}\left(f_{q}\right)=D_{q}\left(f_{q}\right)+\sum_{p \in E_{i}(q)} m_{p q}^{T}\left(f_{q}\right) .
$$

Para cada vértice de entrada, escolhemos um rótulo associado ao custo mínimo para obter um homomorfismo. Para o cálculo de um MCS, aplicamos o mesmo pós-processamento do algoritmo DG, descrito na Seção 2.3.3, desempatando os pares associados a um mesmo vértice do modelo. Para cada vértice do modelo, escolhemos apenas um único vértice da entrada associado ao menor custo, mantendo apenas este par na solução, descartando os demais associados ao mesmo vértice do modelo, indicando que os vértices restantes da entrada estão agora sem classificação.

\subsubsection{Termo de Markov}

Neste trabalho, propomos o seguinte termo de Markov $M\left(f_{p}, f_{q}\right)$ para avaliar as relações espaciais, comparando os vetores codificados nos atributos das arestas da entrada e do modelo, avaliando mudanças de comprimento e de orientação, e penalizando casos que não obedecem a restrição de preservação de arestas, caracterizado por casamentos inexatos, conforme descrito na Seção 2.1.1.

No nosso caso, a restrição de preservação de arestas representa a adjacência entre pontos consecutivos na representação esparsa de objetos, definida para o casamento entre formas (SM) na Seção 3.4.2. Para a colorização assistida por computador (CAC), esta restrição representa a adjacência entre regiões.

O termo de Markov é definido da seguinte maneira:

$$
M\left(f_{p}, f_{q}\right)=\left\{\begin{array}{l}
c_{\mathrm{vec}}\left(\nu_{i}(p, q), \nu_{m}\left(f_{p}, f_{q}\right)\right), \text { se }\left(f_{p}, f_{q}\right) \in E_{m} \\
d, \quad \text { se }\left(f_{p}, f_{q}\right) \notin E_{m} \text { e } f_{p} \neq f_{q}
\end{array}\right.
$$

onde o primeiro caso compara os dois vetores usando a Equação 2.2, e o segundo caso penaliza o custo, adicionando uma constante positiva $d$, incentivando vértices adjacentes a possuírem um mesmo rótulo ${ }^{2}$. Nos experimentos, usamos $d=1$, correspondendo ao valor máximo atribuído pela função de penalidade geométrica definida na Equação 2.2.

\footnotetext{
${ }^{2}$ Bastante similar ao modelo de Potts, usado popularmente como uma restrição de suavidade (smoothness) a problemas low level vision.
} 
No caso de vértices vizinhos com um mesmo rótulo, temos que:

$$
M\left(f_{p}, f_{q}\right)=M(\alpha, \alpha)=c_{\mathrm{vec}}(\nu(p, q), \overrightarrow{0})<d
$$

Assumindo $\theta=0$ para o caso ilustrado na Equação 3.17, a função de penalidade geométrica atribui um valor proporcional a $|\nu(p, q)|$, penalizando vértices distantes, bastante desejável para obter homomorfismos com 'agrupamentos compactos', onde vértices de mesmo rótulo se encontram mais próximos.

Por outro lado, como $M(\alpha, \alpha)$ pode assumir valores diferentes de zero, o termo de Markov proposto não é uma semi-métrica. Neste caso, os métodos baseados em GC, descritos em [18], não garantem boas aproximações. Felizmente, esta limitação não se aplica às técnicas baseadas em BP.

Note que $M$ é uma métrica se satisfaz as três seguintes propriedades:

(m1) $M\left(f_{p}, f_{q}\right)=0 \Leftrightarrow f_{p}=f_{q}$

(m2) $M\left(f_{p}, f_{q}\right)=M\left(f_{q}, f_{p}\right) \geq 0$

(m3) $M\left(f_{p}, f_{q}\right) \leq M\left(f_{p}, f_{r}\right)+M\left(f_{r}, f_{q}\right)$

$M$ é uma semi-métrica se satisfaz as propriedades (m1) e (m2).

\subsubsection{Otimização eficiente via mínima-convolução}

O ponto crítico das abordagens $\mathrm{BP}$, em especial a técnica baseada em máximo-produto, ou equivalentemente mínima-soma, é o cálculo ou atualização das mensagens, definida na Equação 3.14. Felzenszwalb e Huttenlocher [39] propuseram diversas técnicas para diminuir a complexidade de tempo dos algoritmos baseados em BP. Em especial, eles observaram que a atualização das mensagens pode ser expressa como uma mínima-convolução, aplicando esta técnica para termos quadráticos representando diferentes maneiras de avaliar a suavidade (smoothness). As restrições de suavidade são popularmente usadas para problemas low level vision, como cálculo de disparidades de imagens estéreo e restauração de imagens, onde é esperada uma variação suave das intensidades dos pixels no interior das regiões, e uma variação brusca somente nas bordas dos objetos. Neste trabalho, estendemos esta técnica para explorar as relações espaciais através do termo de Markov 
proposto na Seção 3.3.1, permitindo aplicações de 'nível mais alto', como por exemplo, o reconhecimento de objetos via casamento entre formas (shape matching), apresentado na Seção 3.4 .

Primeiramente, reescrevemos a Equação 3.14 através de uma mínima-convolução:

$$
m_{p q}^{t}\left(f_{q}\right)=\min _{f_{p}}\left(h\left(f_{p}\right)+M\left(f_{p}, f_{q}\right)\right)
$$

onde

$$
h\left(f_{p}\right)=D_{p}\left(f_{p}\right)+\sum_{s \in E_{i}(p) \backslash\{q\}} m_{s p}^{t-1}\left(f_{p}\right)
$$

A mínima-convolução é uma operação análoga à convolução discreta padrão, trocando o operador min por somatória, e o operador de soma pelo operador de multiplicação. Conforme observado em [39], apesar de a convolução discreta possuir uma maneira genérica eficiente de ser computada, usando a transformada rápida de Fourier, nenhum resultado genérico é conhecido para mínimas-convoluções. Entretanto, os autores de [39] mostraram maneiras eficientes para computar as mensagens BP em tempo linear, para as restrições de suavidade mais comumente usadas em low level vision, sendo o modelo de Potts o mais simples de todos. Baseado no modelo de Potts, descrito em [39], para calcular uma mensagem de maneira eficiente, assumimos que:

$$
m_{p q}^{t}\left(f_{q}\right)=\min \left(\min _{f_{p}} h\left(f_{p}\right)+d, H\left(f_{q}\right)\right),
$$

onde o termo $H\left(f_{q}\right)$ foi modificado para incluir o termo de Markov, avaliando as relações espaciais:

$$
H\left(f_{q}\right)=\min _{f_{p} \in E_{m}\left(f_{q}\right) \cup\left\{f_{q}\right\}}\left(h\left(f_{p}\right)+M\left(f_{p}, f_{q}\right)\right),
$$

Na Equação 3.21, a adjacência do grafo modelo é explorada de modo a garantir que cada aresta no modelo seja visitada no máximo um número constante de vezes, durante cada atualização do vetor de mensagem, resultando em um algoritmo com complexidade computacional amortizada $O\left(\left|E_{m}\right|\right)$. Note que o próprio rótulo $f_{q}$ também é incluído na avaliação para testar o caso ilustrado na Equação 3.17, não incrementando a complexidade do algoritmo. 
Para as duas aplicações, SM e CAC, foram usados grafos modelos planares, onde $\left|E_{m}\right|=O\left(\left|V_{m}\right|\right)$. Desta forma, cada atualização de vetor de mensagem possui uma complexidade de tempo $O\left(\left|V_{m}\right|\right)$. Como este cálculo é repetido para cada um dos vértices de entrada, o método BP proposto possui um consumo de tempo $O\left(\left|V_{i}\right| \cdot\left|V_{m}\right|\right)=O\left(n^{2}\right)$, $n=\max \left\{\left|V_{i}\right|,\left|V_{m}\right|\right\}$, executando $T$ iterações, $T$ constante.

\subsection{Aplicação 1: casamento entre formas}

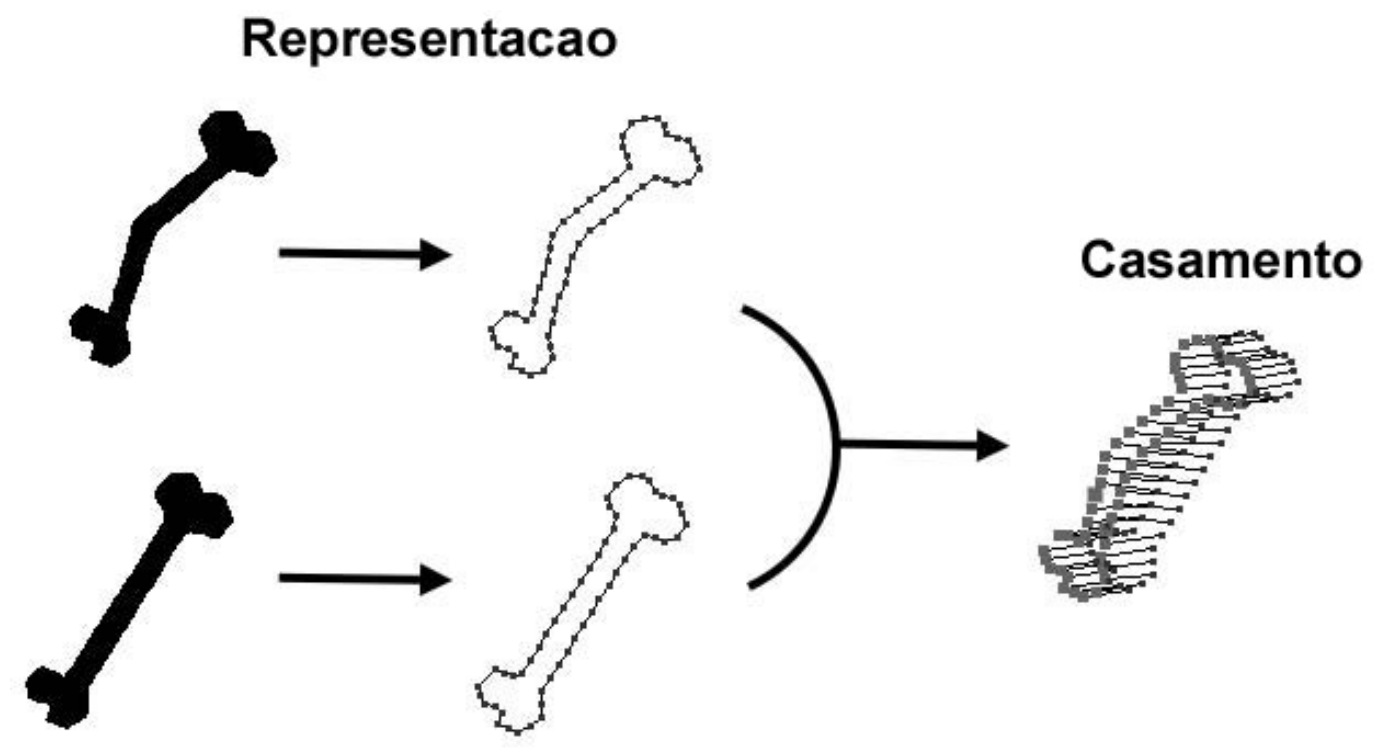

Figura 3.1: Resumo de nossa abordagem para casamento entre formas.

Forma (shape) é uma informação fundamental para consultas em bancos de dados baseados em imagens. Grandes esforços de pesquisa têm sido realizados para desenvolver técnicas de similaridade entre as formas, por exemplo usando as silhuetas dos objetos [91]. Estratégias mais genéricas representam as formas como um conjunto de pontos, considerando, além dos contornos externos de silhueta, também os contornos 'internos' dos objetos, fornecendo mais informações sobre a aparência, por exemplo, informações de textura. Neste trabalho, seguimos a segunda abordagem, onde um objeto é representado por um conjunto de pontos, e a sua forma é representada por um padrão discreto de pontos, amostrados nas bordas do objeto obtidas por um detector, por exemplo, Canny. O objetivo do casamento entre formas (shape matching, SM) [63] é encontrar um MCS 
entre dois padrões de pontos, através de representações compactas dos objetos para obter eficiência computacional.

O método proposto explora com sucesso representações esparsas para as formas, usando o termo de Markov definido na Seção 3.3.1, combinado com uma nova distância, usada para mensurar a dissimilaridade entre as formas, proposta na Seção 3.4.3. Resultados bastante satisfatórios são ilustrados nas Seções 3.4.4 e 3.4.5, usando bancos de imagens bem conhecidos na literatura, como as bases de dados de silhuetas de Kimia [82] e de MPEG-7 [51], e os bancos de imagens COIL [61] e MNIST [52], para reconhecimento de objetos 3D e de dígitos manuscritos.

\subsubsection{Histórico}

Trabalhos fortemente relacionados ao nosso são devido a Belongie et al. [9] e Torresani et al. [86]. Inspirados no trabalho descrito em [9], usamos shape contexts como informações de aparência. Para a estrutura, exploramos as relações espaciais em termos de adjacência, distância e orientação entre os pontos, usando o termo de Markov descrito na Seção 3.3.1. Os autores de [86] também exploraram estes três aspectos de relações espaciais, mas formulados em uma complexa expressão de energia, otimizada por uma sofisticada técnica de decomposição dual baseada em busca exaustiva por subproblemas locais, resultando em um método de alto custo computacional. Por exemplo, o método resultante foi testado para uma pequena fração do banco de dados MNIST. No nosso caso, usamos uma formulação mais simples, baseada em atribuição quadrática, representando uma energia mais simples, definida na Equação 3.13, possibilitando a aplicação do método BP sobre o banco MNIST completo.

Recentemente, técnicas de aprendizado, por exemplo [27], têm sido propostas para melhorar a qualidade da classificação, exigindo algoritmos eficientes para o problema SM. Nesta Tese, exploramos as relações espaciais de maneira simples e eficiente, combinando uma formulação de atribuição quadrática baseada em MRFs e o uso de representações compactas para as formas, resultando em um algoritmo BP eficiente, com complexidade computacional quadrática, conforme descrito na Seção 3.3.2. 


\subsubsection{Representação esparsa para as formas}

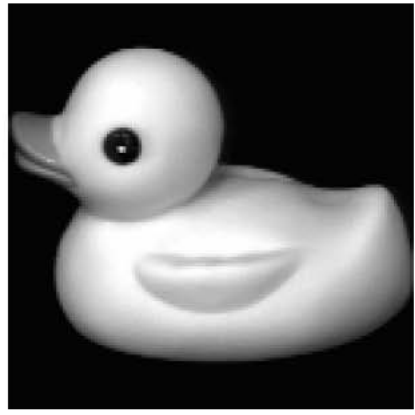

(a)

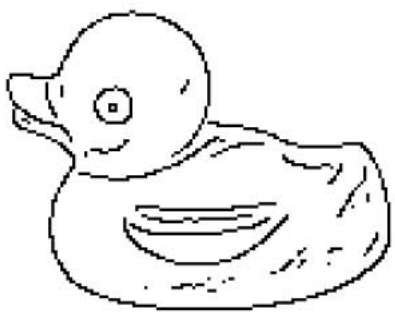

(b)

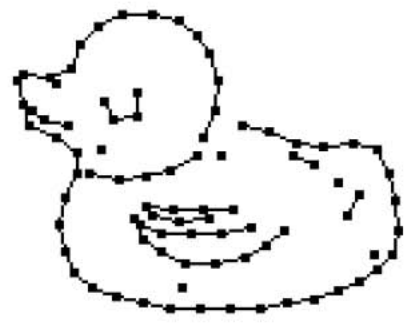

(c)

Figura 3.2: (a) Objeto. (b) Bordas obtidas pelo detector Canny. (c) Grafo esparso representando (b).

Para cada grafo, amostramos de maneira grosseira pontos uniformemente espaçados nas bordas de um objeto, representando cada ponto por um vértice. Similarmente a [38], contornos poligonais foram usados para aproximar os contornos originais do objeto, conforme ilustrado na Figura 3.2(c). Entretanto, o autor de [38] usou uma triangularização dos pontos amostrados na silhueta do objeto para permitir uma sofisticada decomposição em partes deformáveis. Aqui, usamos uma representação mais simples, mas não restrita a silhuetas.

\subsubsection{Distância entre formas}

Para operacionalizar a noção de similaridade entre as formas representadas pelos dois grafos, propomos a seguinte distância:

$$
d_{\text {shape }}\left(G_{i}, G_{m}\right)=\sum_{p \in V_{i}: f_{p} \neq \text { NULL }} b_{p}\left(f_{p}\right)+\sum_{p \in V_{i}: f_{p}=\text { NULL }} \Lambda_{p}\left(f_{p}\right)
$$

onde o primeiro termo é a soma das confianças computadas pela Equação 3.15, considerando apenas os vértices de entrada rotulados. Já os vértices não rotulados são penalizados pelo segundo termo, atribuindo o custo máximo $\Lambda_{p}\left(f_{p}\right)=\max _{p \in V_{i}: f_{p} \neq \mathrm{NULL}}\left\{b_{p}\left(f_{p}\right)\right\}$ a cada vértice sem classificação. 
A distância proposta acima foi usada para classificar objetos 3D e dígitos manuscritos, usando dados de COIL [61] e de MNIST [52], respectivamente. Para completar a definição de nossa abordagem baseada em BP para o problema SM, usamos a distância entre SCs definida na Equação 2.6, Seção 2.4.3, no papel do termo linear da Equação 3.13.

\subsubsection{Experimentos usando silhuetas}

O método proposto foi testado com silhuetas, por exemplo, usando imagens de Kimia [82] e de MPEG-7 [51], conforme ilustrado nas Figuras 3.3 e 3.4. Neste caso, foi feita uma comparação qualitativa com dois algoritmos: graduated assigment (GA) [29], conhecido por ser extremamente robusto a deformações não-rígidas, e o método Húngaro para BGM [68], usando apenas as informações de aparência, SCs, para ilustrar a qualidade de nossa abordagem e a importância das relações espaciais. Conforme descrito na Seção 2.4.4 e Equação 2.7, para rejeitar possíveis outliers, usamos uma matriz de dissimilaridade expandida $C_{\varepsilon}=\left[\begin{array}{ll}C & \varepsilon\end{array}\right]$, de tamanho $2 \times \max \left\{\left|V_{i}\right|,\left|V_{m}\right|\right\}$ para o método Húngaro, BGM.

A Figura 3.5 ilustra alguns casos onde as informações de SC estão bastante deterioradas devido a grandes deformações entre as formas. Neste caso, o algoritmo BGM produziu resultados insatisfatórios, com um grande número de 'arestas cruzadas', indicando um grande número de correspondências equivocadas. Em nossa abordagem, as relações espaciais entre os pontos consecutivos, amostrados na borda do objeto, são usadas para compensar a pouca discriminação proporcionada pelos SCs, ajudando a produzir resultados comparáveis ao do método GA. Em todas as figuras, os pontos em destaque representam vértices da entrada sem correspondência.

Usando um $\varepsilon$ maior que o máximo das distâncias entre os SCs, o método BGM casa o maior número possível de vértices. Neste caso, BGM descarta vértices da entrada como outliers somente se $\left|V_{i}\right|>\left|V_{m}\right|$. Variando $\varepsilon$ com valores menores, mais vértices são descartados, mas em geral isso não garante eliminar as 'arestas cruzadas', conforme ilustrado na Figura 3.6. Em nossa abordagem baseada em BP, vértices da entrada são descartados pelo desempate de vértices, convertendo o homomorfismo obtido para um MCS, conforme descrito no final da Seção 3.3. Com este simples pós-processamento, obtivemos resultados comparáveis aos do algoritmo GA, conforme exibido na Figura 3.5.

Apesar do algoritmo GA ser bastante robusto a deformações, ilustramos na Figura 3.7 

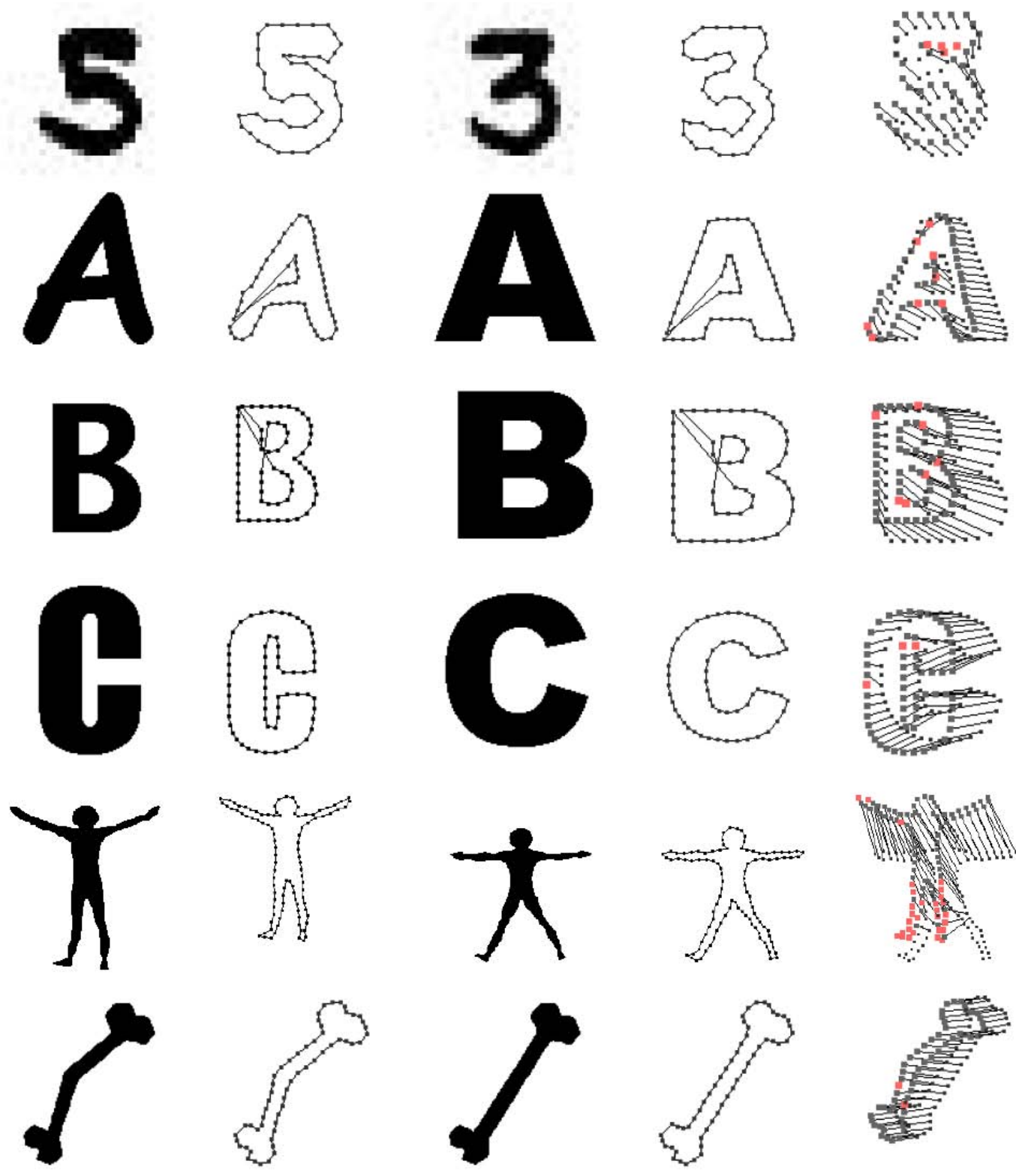

(a)

(b)

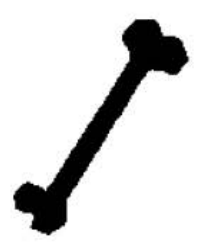

(c)

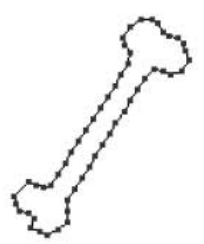

(d)

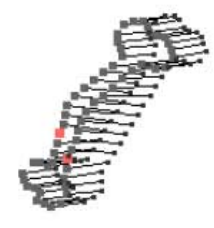

(e)

Figura 3.3: Exemplos de SM usando letras com diferentes fontes, dígitos manuscritos de MNIST [52], silhueta humana de [48] e silhuetas de Kimia [82]. (a) Silhueta de entrada.
(b) Grafo de entrada.
(c) Silhueta do modelo.
(d) Grafo modelo.
(e) Resultado de casamento usando nossa abordagem baseada em BP. 

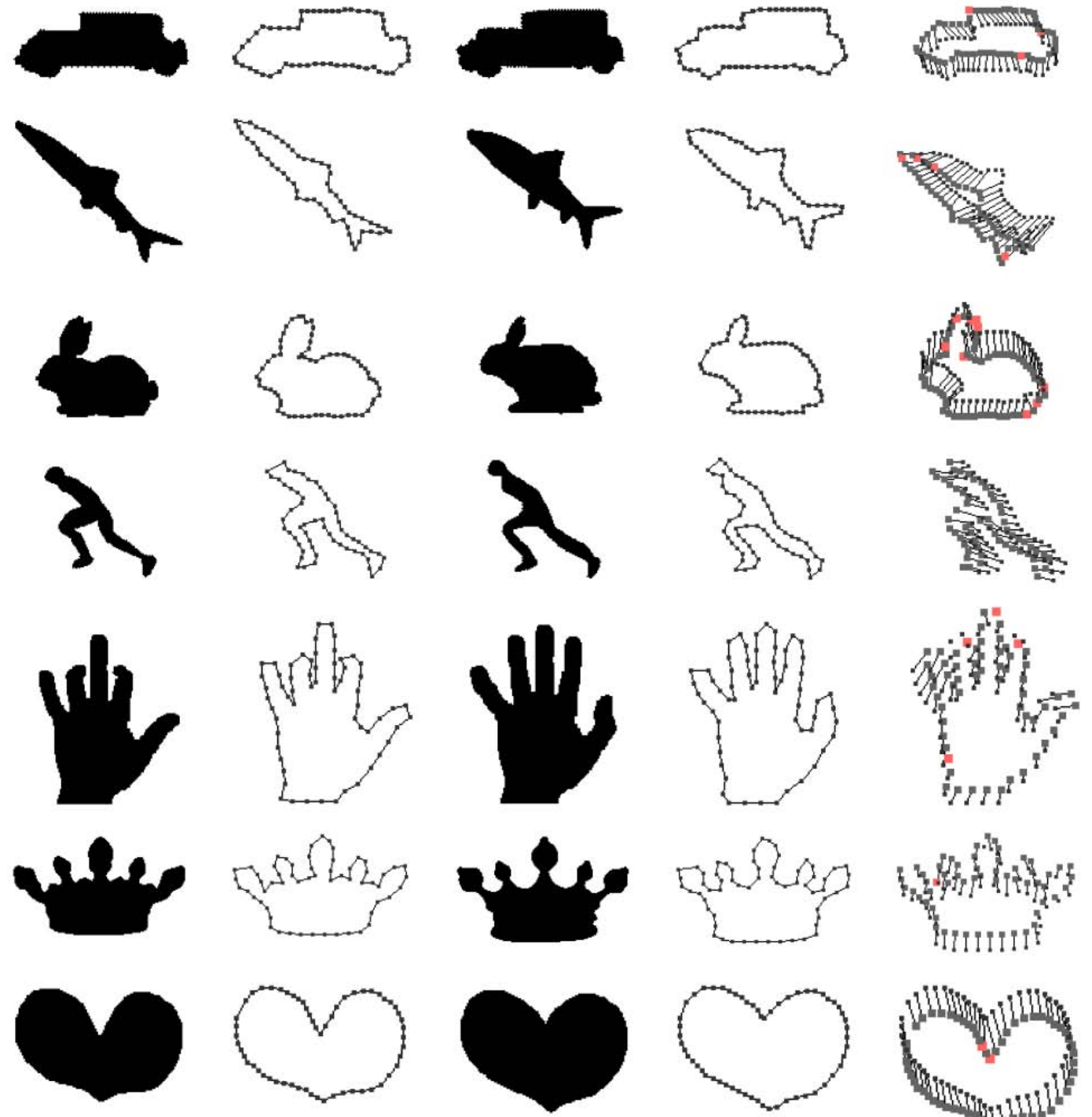

(a)

(b)

(c)

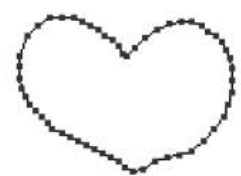

(d)

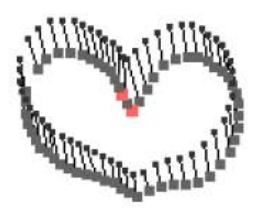

(e)

Figura 3.4: Exemplos de SM usando silhuetas de Kimia [82]. (a) Silhueta de entrada. (b) Grafo de entrada. (c) Silhueta do modelo. (d) Grafo modelo. (e) Resultado de casamento usando nossa abordagem baseada em BP. 

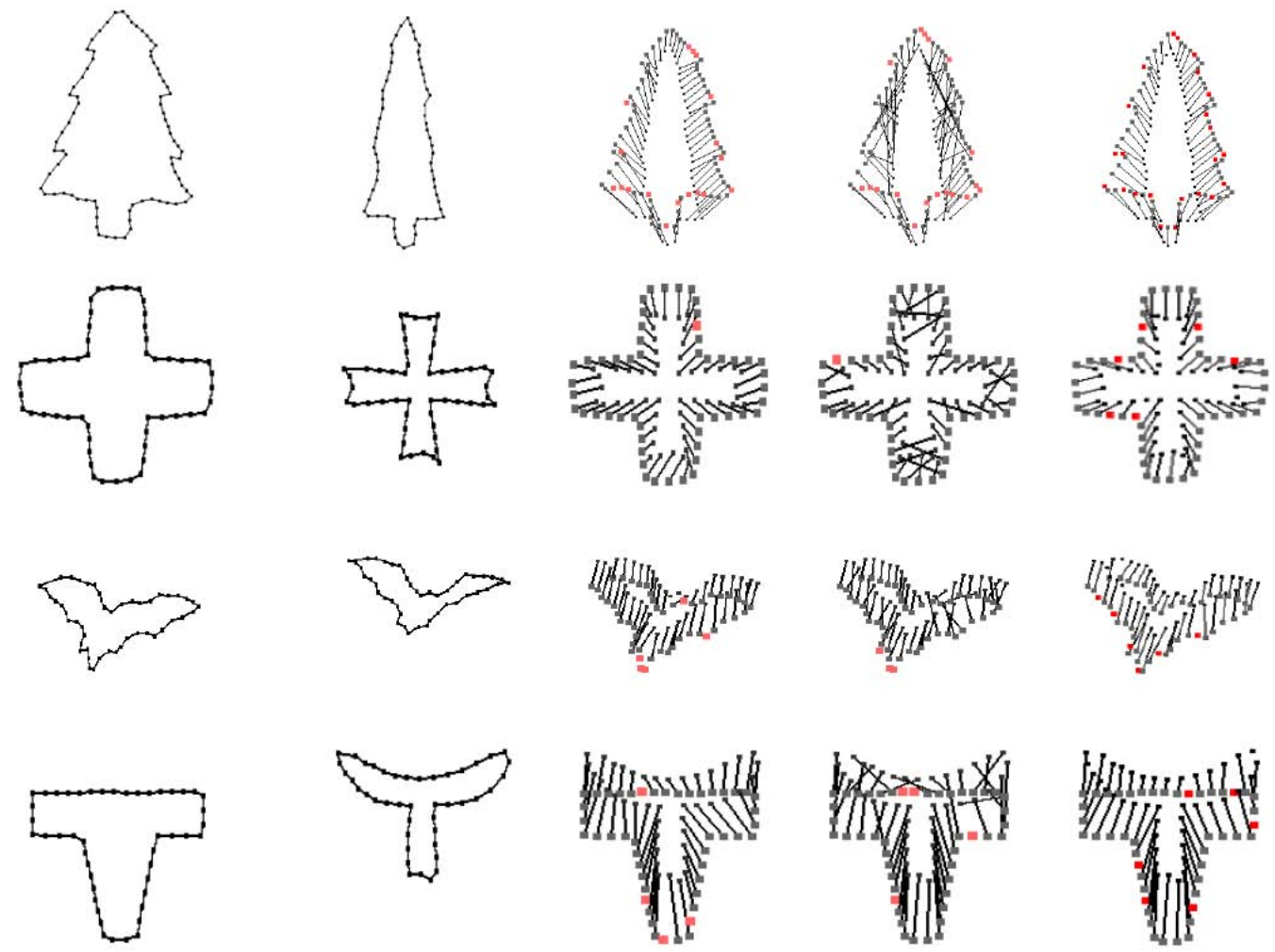

$\left(G_{i}\right)$

$\left(G_{m}\right)$

(BP)
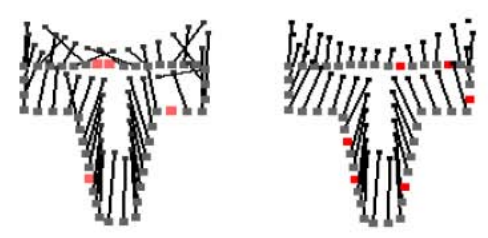

(BGM)

(GA)

Figura 3.5: Exemplos de SM comparando nossa abordagem baseada em BP, o método Húngaro (BGM), e graduated assignment (GA) [29].

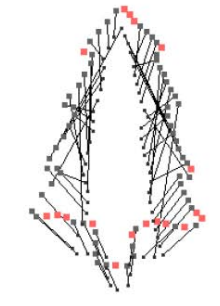

$$
(\varepsilon=0.5)
$$

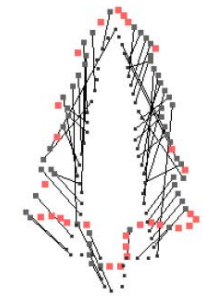

$(\varepsilon=0.4)$

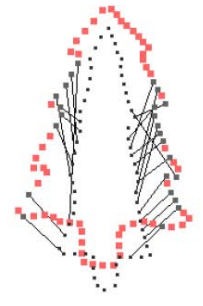

$(\varepsilon=0.3)$

Figura 3.6: Em geral, variar $\varepsilon$ não resulta em eliminação dos cruzamentos de arestas pelo método BGM. 


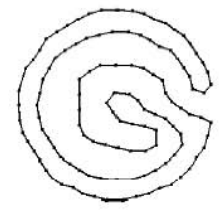

$\left(G_{i}\right)$

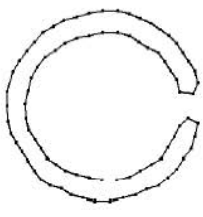

$\left(G_{m}\right)$

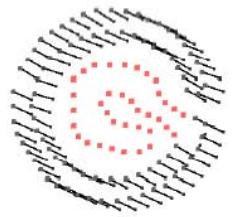

$(\mathrm{BP})$
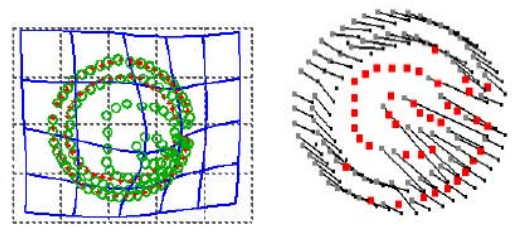

$(\mathrm{GA})$

Figura 3.7: Um exemplo onde o método baseado em BP encontra corretamente o modelo $G_{m}$ imerso na entrada $G_{i}$. Por outro lado, GA tenta transformar todos os pontos, incluindo outliers, produzindo um resultado insatisfatório.

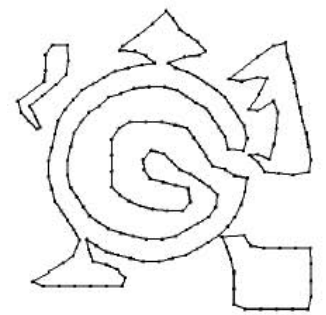

$\left(G_{i}\right)$

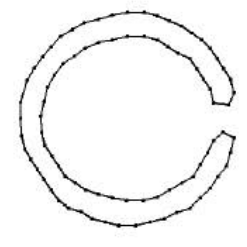

$\left(G_{m}\right)$

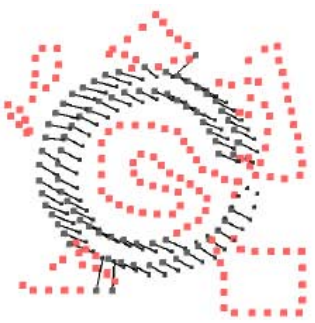

$(\mathrm{BP})$

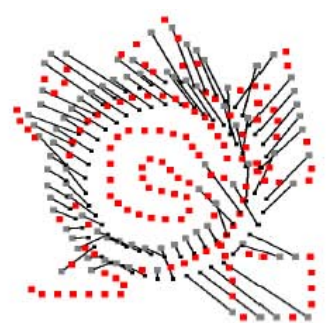

(GA)

Figura 3.8: Um exemplo de clutter, onde a entrada representa uma versão bastante ruidosa do modelo. Nosso resultado foi bastante satisfatório, em contraste com o resultado pobre obtido por GA. 
uma situação simples em que nossa abordagem BP supera GA. Como nossa formulação é um 'mapeamento da cena para o modelo', conforme descrito na Seção 3.1.2, isso permite procurar por ocorrências do modelo imersas na cena. Na Figura 3.7, o método BP encontra corretamente o modelo $G_{m}$ imerso na entrada $G_{i}$, representando a cena. Neste caso, o modelo representa uma visão parcial da cena. Por outro lado, o método GA produz um resultado insatisfatório, produzindo correspondências incorretas, devido ao fraco poder discriminativo da distância Euclidiana e a incapacidade das transformações TPS de suprirem essa falta de informações de aparência. Os benefícios de nossa abordagem são mais evidentes na presença de clutter, conforme exibido na Figura 3.8.

\subsubsection{Experimentos usando MNIST e COIL}

Similarmente a [9], nosso método se encontra na categoria dos classificadores baseados em protótipos, onde classes são representadas por exemplos ideais, não por um conjunto de regras lógicas formais. A classificação ou reconhecimento baseado em protótipos pode ser diretamente traduzido para o arcabouço computacional que inclui métodos baseados no vizinho mais próximo (nearest-neighbor, $\mathrm{NN}$ ), usando múltiplas visões de cada objeto a ser classificado. Por exemplo, através de classificadores 1-NN, a classificação é determinada pelo protótipo ou pela visão mais próxima, usando a distância definida na Equação 3.22. Para classificadores $K-\mathrm{NN}$, a classificação é dada pela classe com maior votação dentre os $K$ protótipos mais próximos.

Neste contexto, usando classificadores 1-NN, é importante estudar a performance para diferentes valores de $n$, especialmente para valores pequenos de $n$, onde $n$ é o número de protótipos, ou visões, ou exemplos no conjunto de treinamento. Para classificadores $K$ NN com um conjunto de treinamento de tamanho fixo, é importante analisar a robustez de acordo com diferentes valores de $K$.

Estas duas questões são tratadas neste experimento, dividida em duas partes. A primeira usa a base de imagens COIL [61] para o reconhecimento de objetos 3D. Neste caso, testamos nosso classificador 1-NN usando conjuntos de protótipos de diferentes tamanhos, obtidos por visões amostradas, igualmente espaçadas. A segunda parte usa a base MNIST [52] para o reconhecimento de dígitos manuscritos, onde testamos nossa abordagem $K$-NN para diferentes valores de $K$. 


\section{COIL}

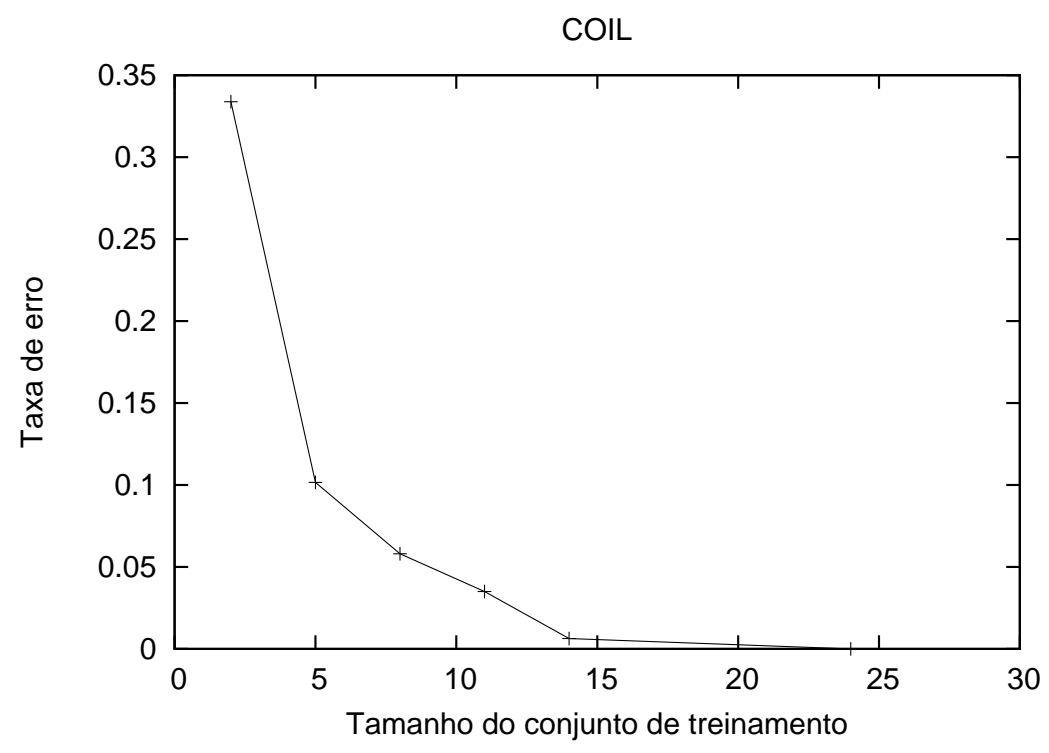

Figura 3.9: Taxas de erros para o reconhecimento de objetos 3D, usando o classificador 1-NN para diferentes tamanhos de conjuntos de treinamento.

O banco de imagens COIL [61] envolve 20 objetos encontrados popularmente em uma residência. Cada objeto foi colocado em uma plataforma rotacionável e fotografado a cada $5^{\circ}$. Esta base de dados inclui 70 visões distintas por objeto. Testamos nosso classificador 1-NN para diferentes conjuntos de treinamentos, definidos selecionando-se visões igualmente espaçadas. A Figura 3.9 ilustra o comportamento do nosso método, onde a taxa de erro decresce conforme o número de protótipos aumenta, concordando com o esperado. Por exemplo, usando 8 visões igualmente espaçadas por objeto, a taxa de erro obtida foi de 0.0581 .

Além disso, em abordagens baseadas em protótipos, diferentes classes ou categorias necessitam diferentes quantidades de protótipos ou visões, dependendo da complexidade de cada objeto. Usando uma estratégia de agrupamento baseada em $k$-medoids, seguimos de maneira similar a abordagem descrita em [9] para uma escolha mais adequada de protótipos.

$k$-medoids é um método de agrupamento para particionar $n$ observações em $k$ grupos (clusters), onde cada observação pertence ao grupo com o protótipo mais próximo. 


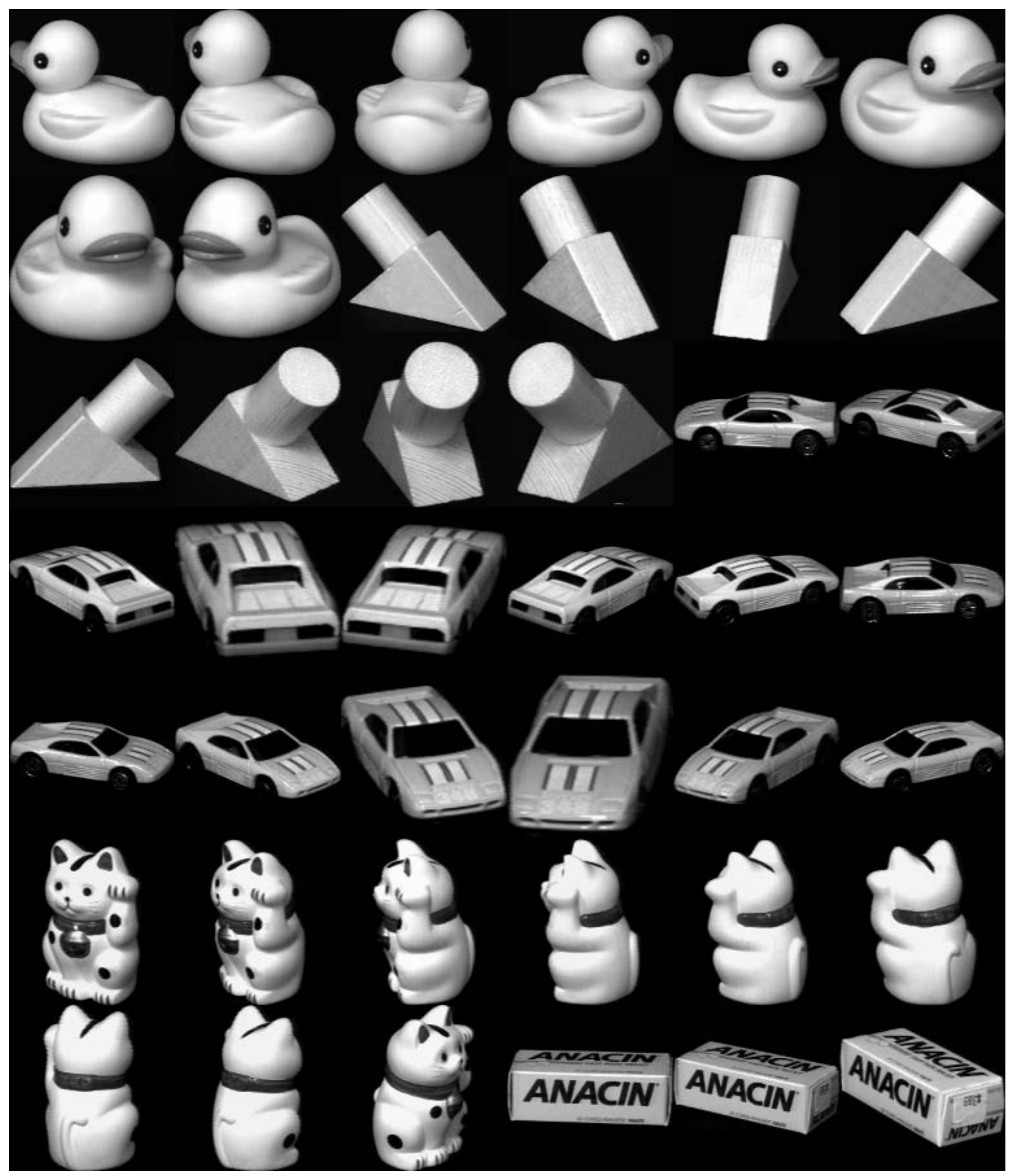

Figura 3.10: Usando 158 protótipos do banco de imagens COIL [61], nossa abordagem BP produziu 20 erros de um total de 1242 classificações. 


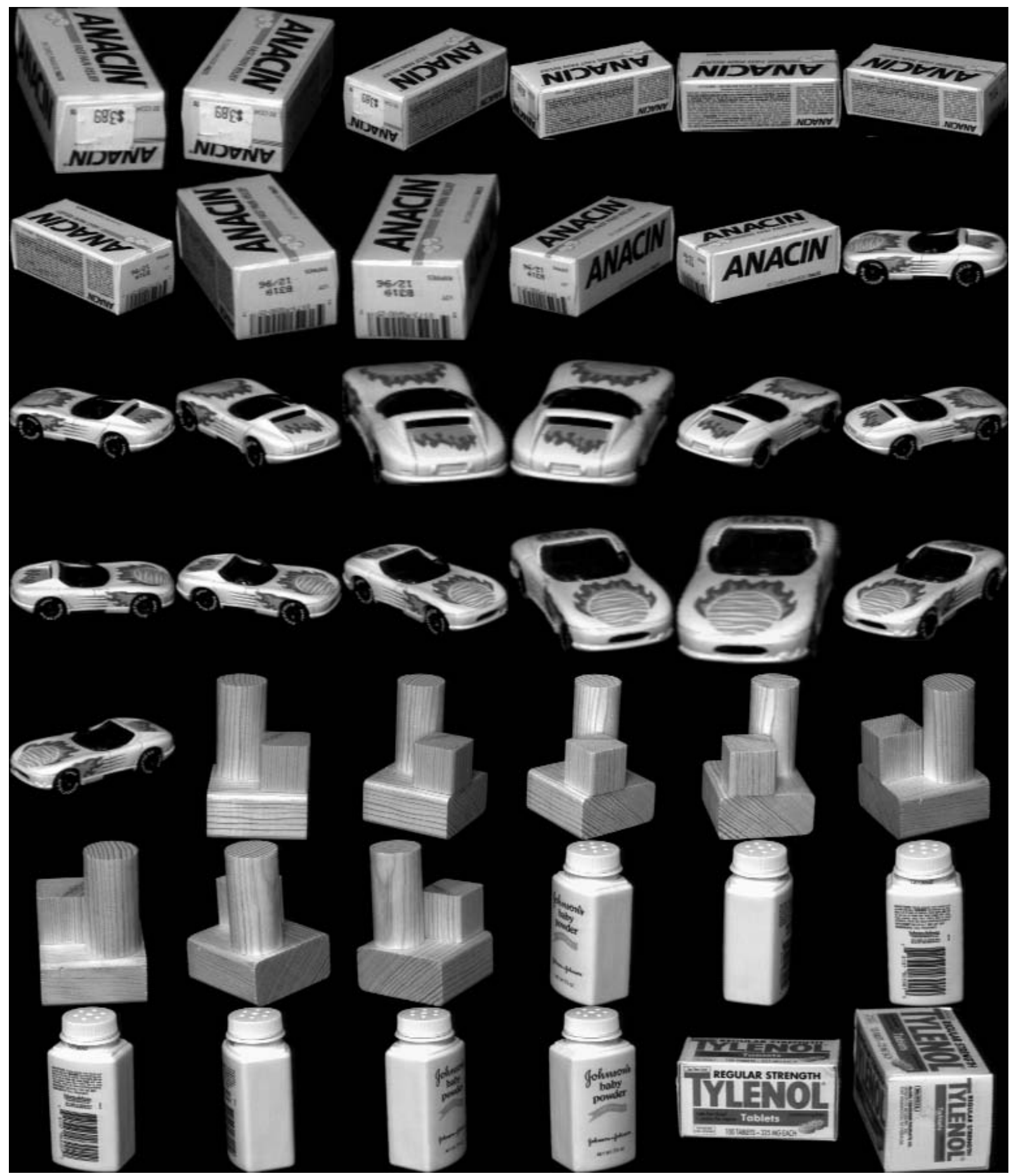

Figura 3.11: Usando 158 protótipos do banco de imagens COIL [61], nossa abordagem BP produziu 20 erros de um total de 1242 classificações. 


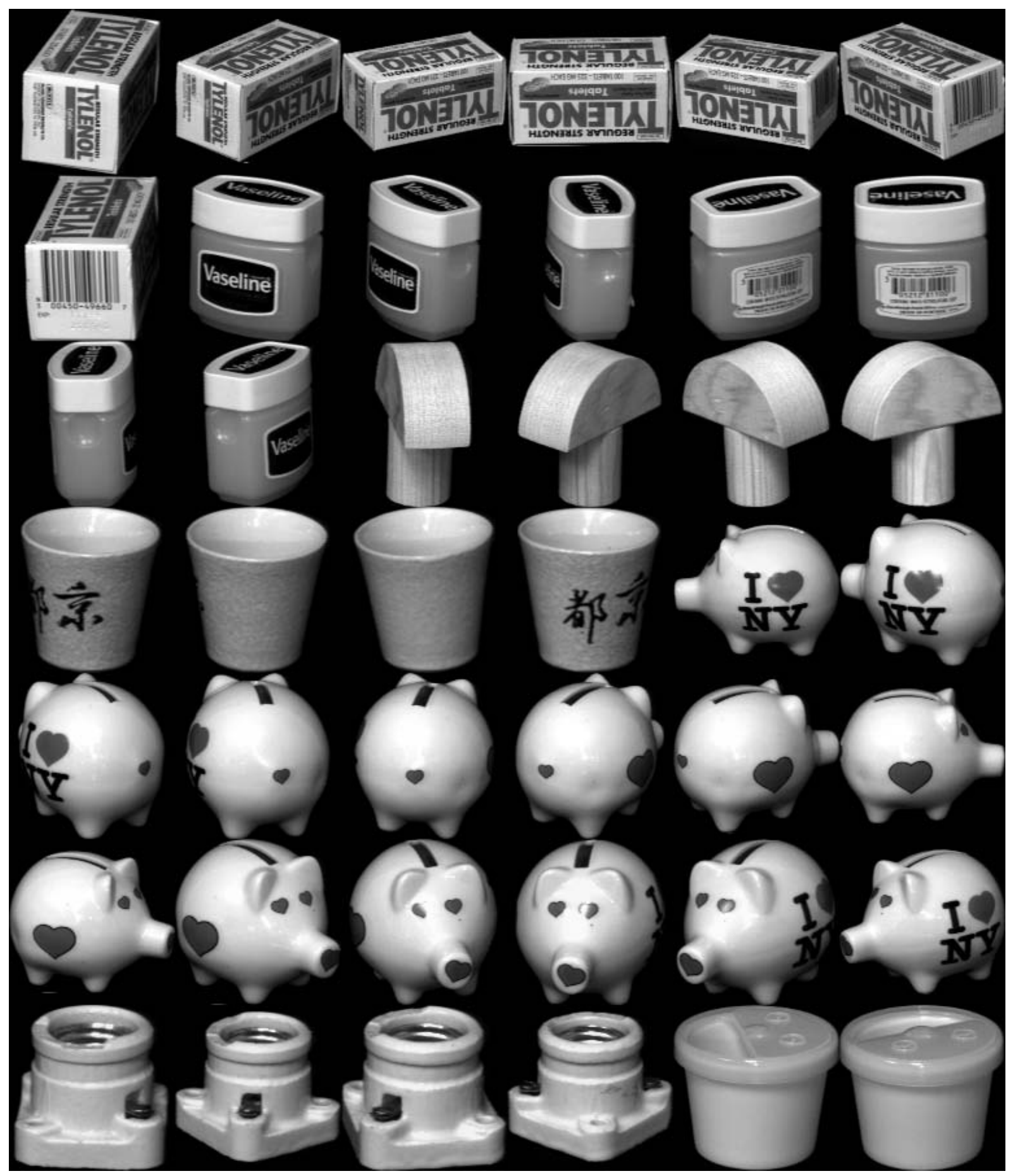

Figura 3.12: Usando 158 protótipos do banco de imagens COIL [61], nossa abordagem BP produziu 20 erros de um total de 1242 classificações. 


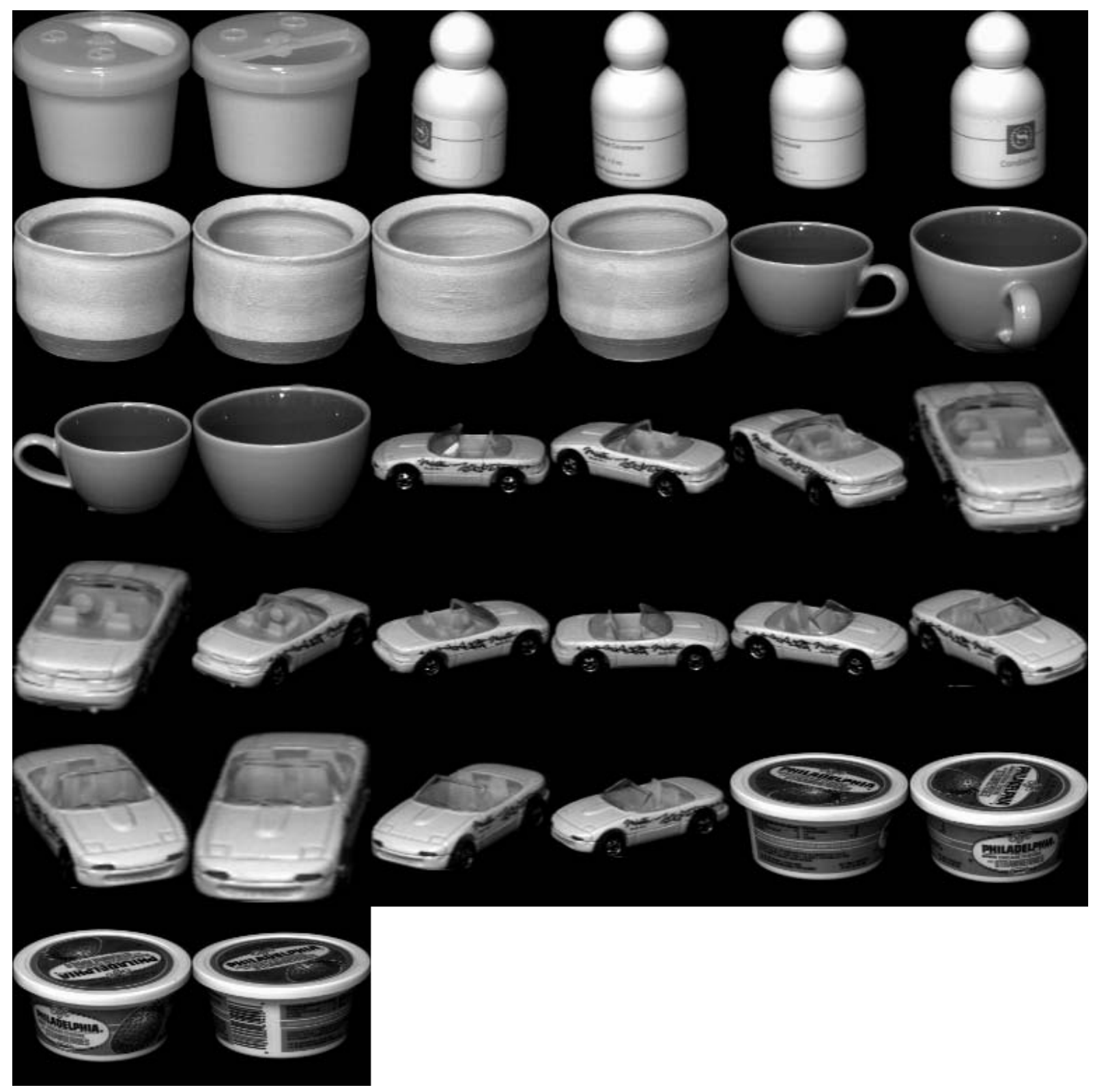

Figura 3.13: Usando 158 protótipos do banco de imagens COIL [61], nossa abordagem BP produziu 20 erros de um total de 1242 classificações. 

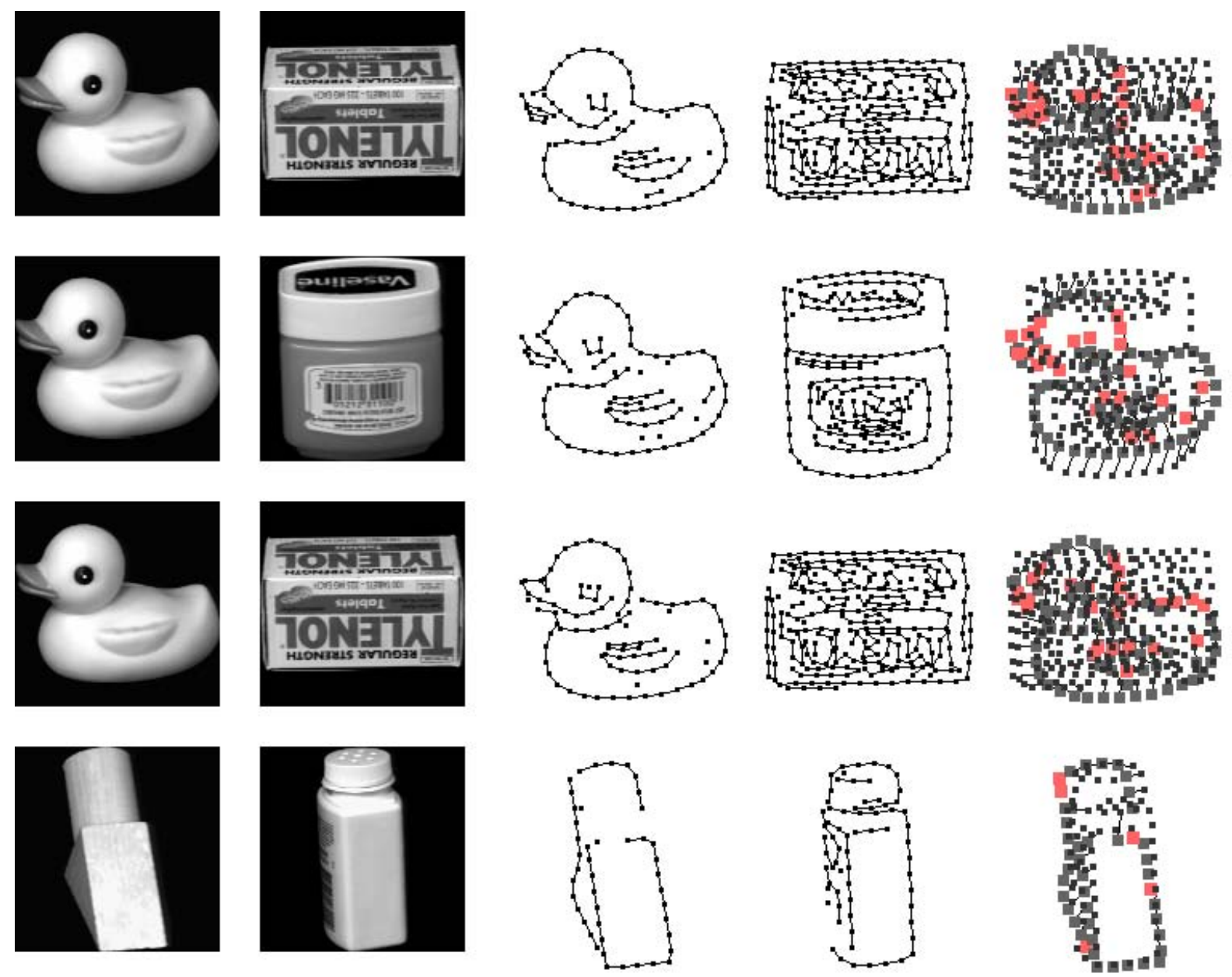

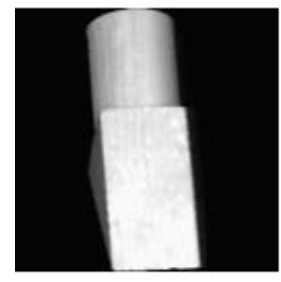

(a)

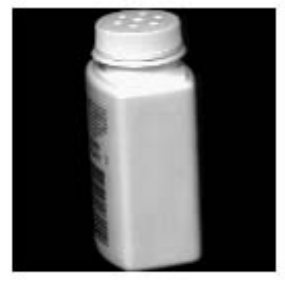

(b)

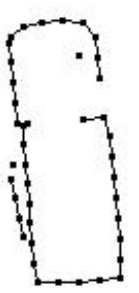

(c)

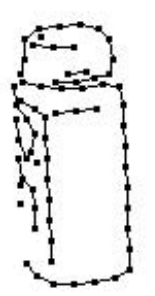

(d)

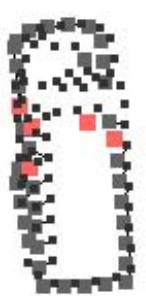

(e)

Figura 3.14: Classificações incorretas produzidas pelo nosso classificador 1-NN, usando o banco de imagens COIL [61]. (a) Image de entrada. (b) Vizinho mais próximo, de acordo com a distância definida na Equação 3.22. (c) Grafo esparso representando (a), conforme descrito na Seção 3.4.2. (d) Similarmente, grafo esparso representando (b). (e) Casamento entre formas, onde os vértices de entrada sem correspondência estão destacados. 

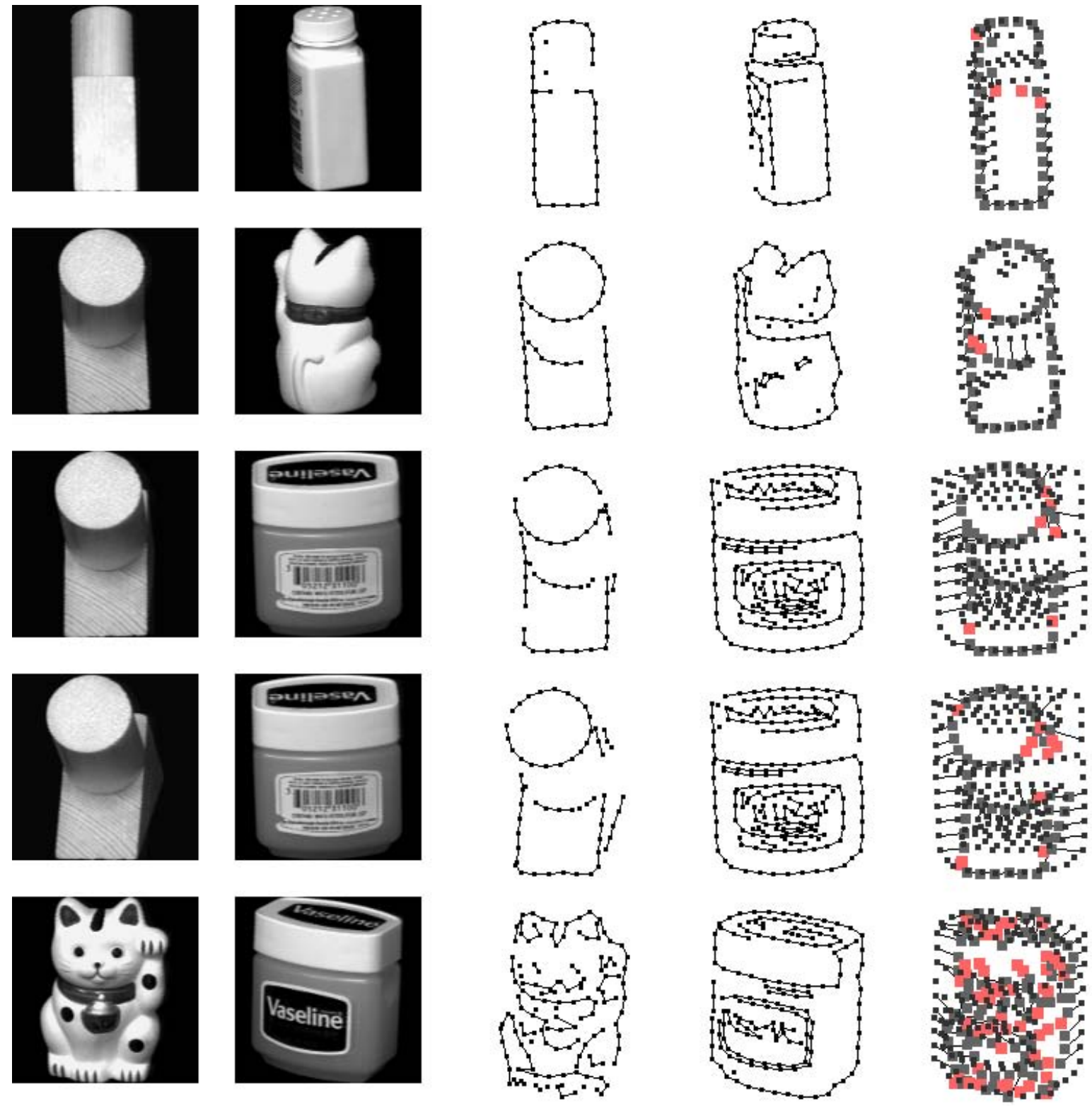

(a)

(b)

(c)

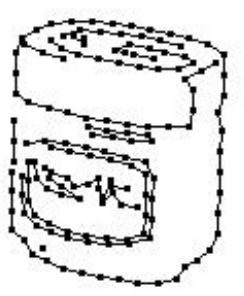

(d)

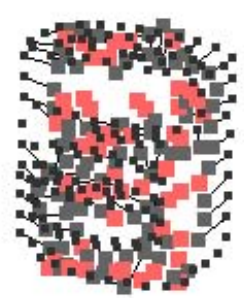

(e)

Figura 3.15: Classificações incorretas produzidas pelo nosso classificador 1-NN, usando o banco de imagens COIL [61]. (a) Image de entrada. (b) Vizinho mais próximo, de acordo com a distância definida na Equação 3.22. (c) Grafo esparso representando (a), conforme descrito na Seção 3.4.2. (d) Similarmente, grafo esparso representando (b). (e) Casamento entre formas, onde os vértices de entrada sem correspondência estão destacados. 

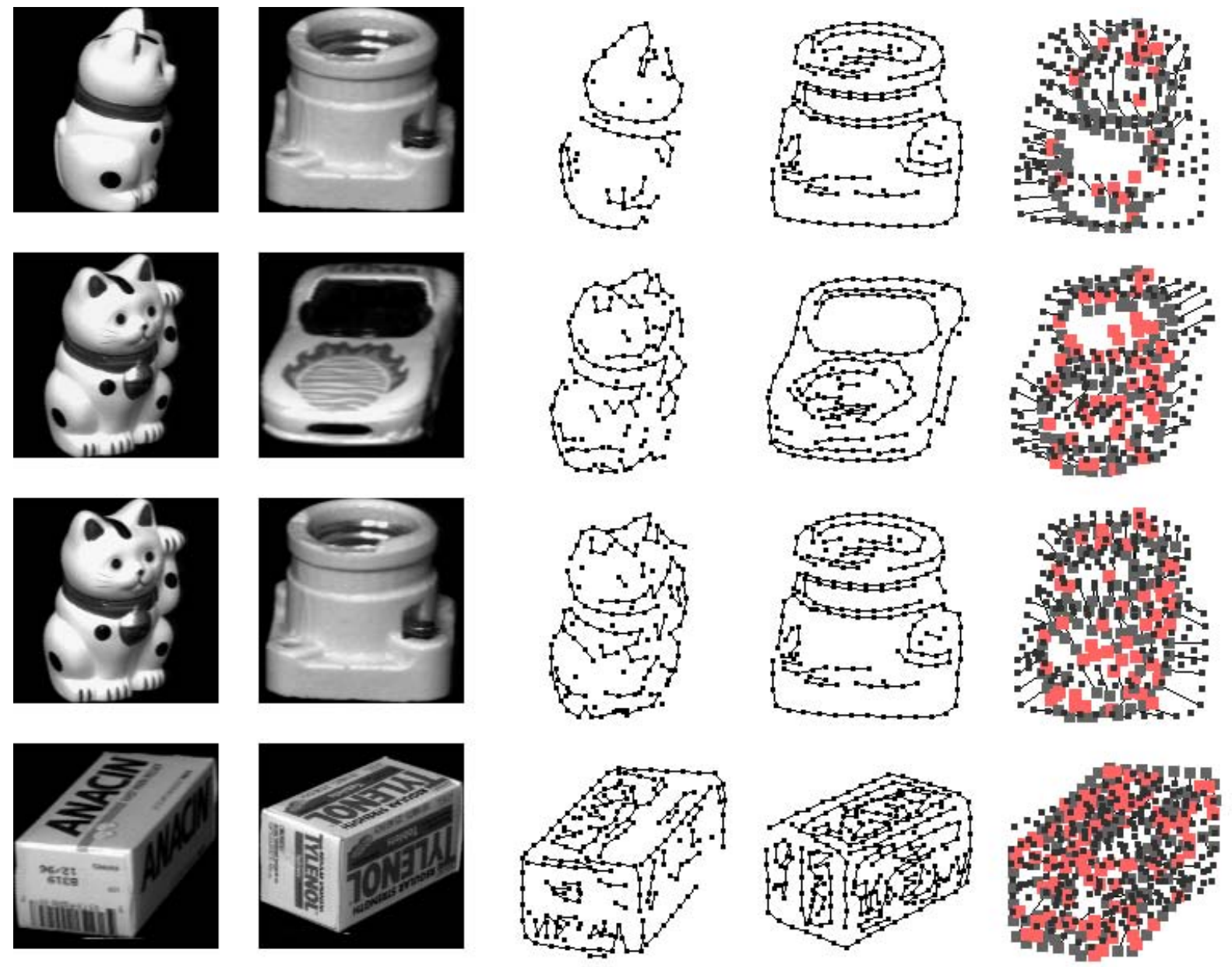

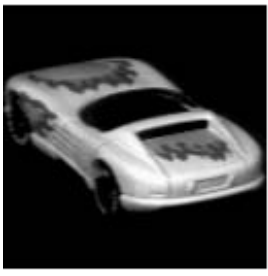

(a)

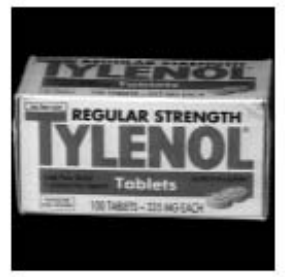

(b)

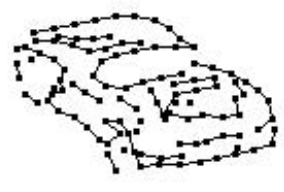

(c)

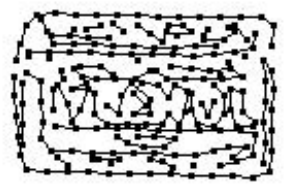

(d)

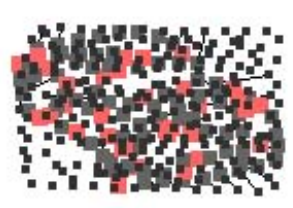

(e)

Figura 3.16: Classificações incorretas produzidas pelo nosso classificador 1-NN, usando o banco de imagens COIL [61]. (a) Image de entrada. (b) Vizinho mais próximo, de acordo com a distância definida na Equação 3.22. (c) Grafo esparso representando (a), conforme descrito na Seção 3.4.2. (d) Similarmente, grafo esparso representando (b). (e) Casamento entre formas, onde os vértices de entrada sem correspondência estão destacados. 

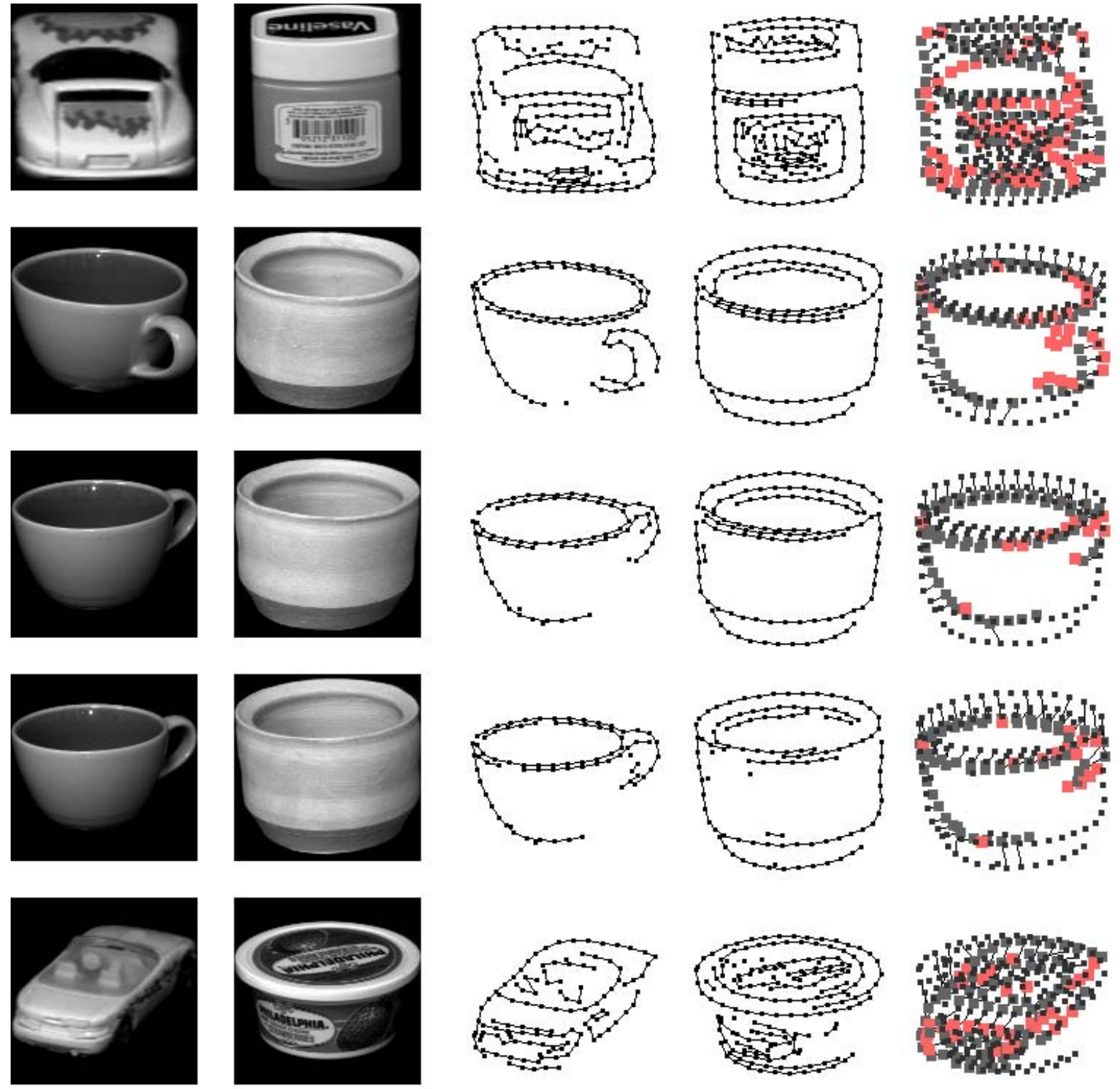

(a)

(b)

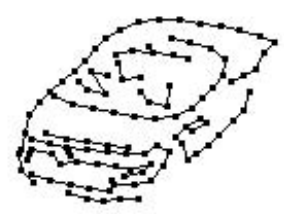

(c)

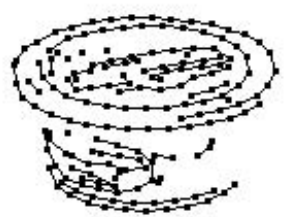

(d)

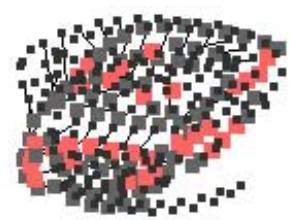

(e)

Figura 3.17: Classificações incorretas produzidas pelo nosso classificador 1-NN, usando o banco de imagens COIL [61]. (a) Image de entrada. (b) Vizinho mais próximo, de acordo com a distância definida na Equação 3.22. (c) Grafo esparso representando (a), conforme descrito na Seção 3.4.2. (d) Similarmente, grafo esparso representando (b). (e) Casamento entre formas, onde os vértices de entrada sem correspondência estão destacados. 
Os protótipos são chamados de medoids, representando observações que minimizam a distância média no grupo.

Através de $k$-medoids, melhoramos a taxa de erro de 0.0581 para 0.0161 , usando 158 protótipos (Figuras 3.10 a 3.13, uma média de 8 protótipos por objeto), resultando em 20 erros de um total de 1242 classificações. Por exemplo, esta taxa é menor que a taxa de erro obtida em [9], que foi de 0.0240 usando uma média de 4 protótipos por objeto. As Figuras 3.14 a 3.17 exibem todos os 20 erros obtidos pelo nosso método combinado com $k$-medoids. Por exemplo, nosso método falha quando há um grande número de rótulos não utilizados, ou seja, vértices do modelo sem correspondência. Note que este caso viola a hipótese assumida de que o modelo está imerso na cena, e a Equação 3.22 não penaliza os rótulos não utilizados.

\section{MNIST}

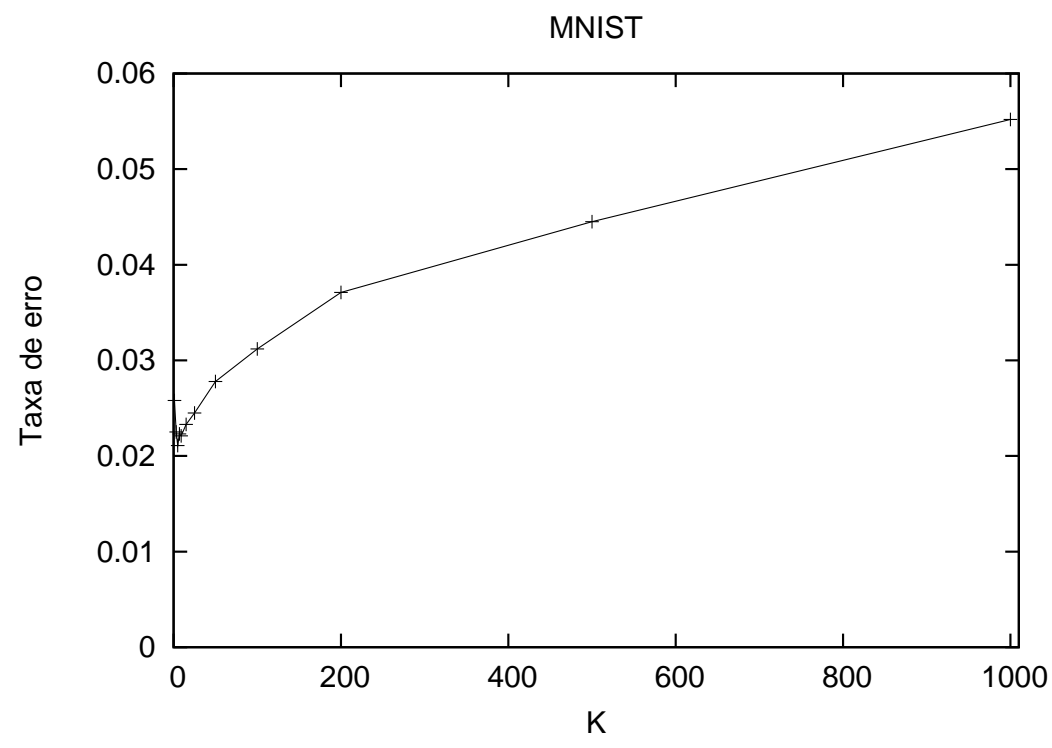

Figura 3.18: Taxas de erros para o reconhecimento de dígitos manuscritos, usando classificadores $K$-NN para diferentes valores de $K$.

O banco de dados MNIST [52] consiste de 60000 dados de treinamento e 10000 dados de teste, representando dígitos manuscritos. No sítio http://yann.lecun.com/exdb/ mnist/, é feita uma comparação entre mais de 60 algoritmos, cujas taxas de erro variam 


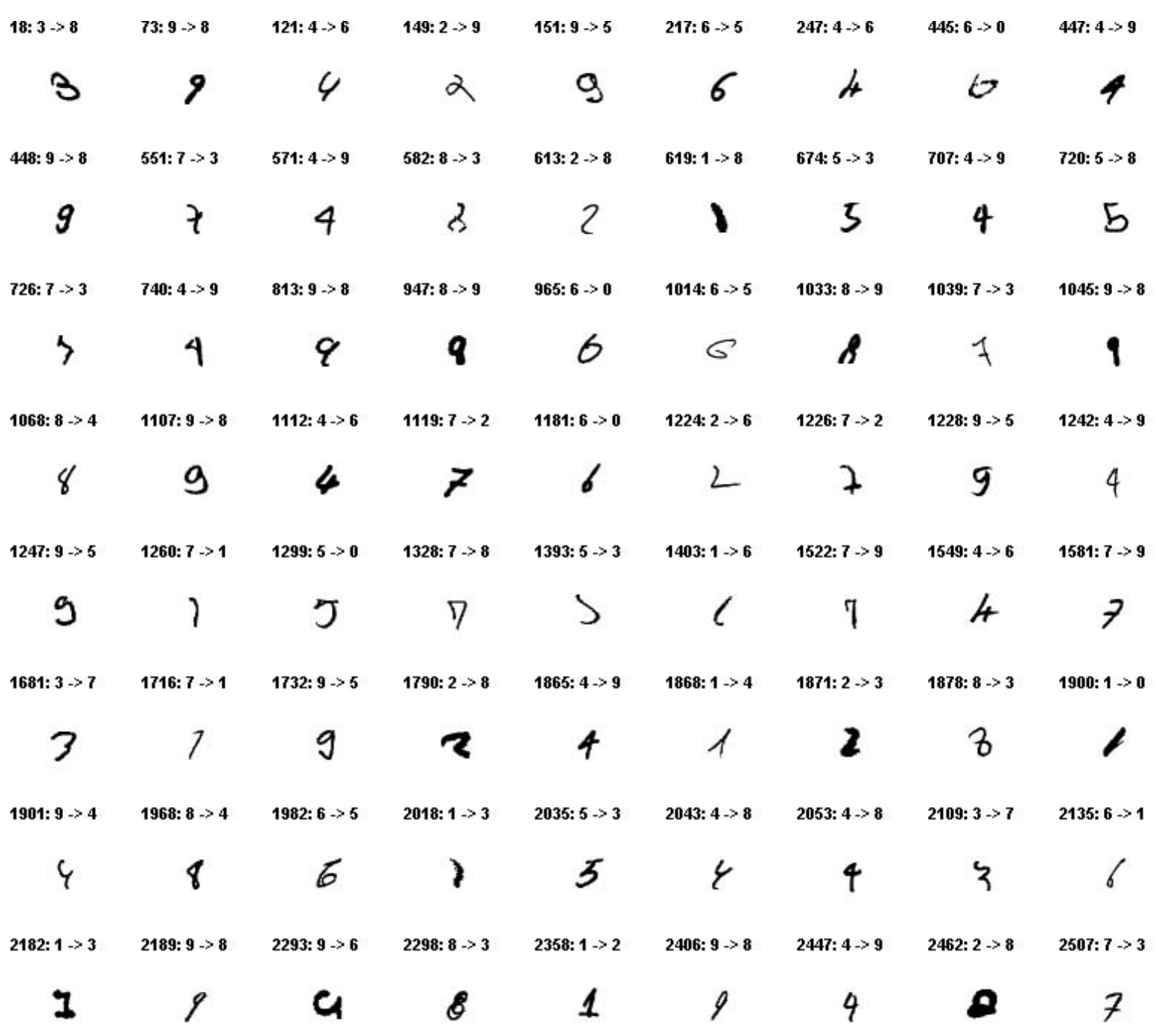

Figura 3.19: Classificações incorretas produzidas pelo nosso classificador 5-NN, usando o banco de imagens MNIST [52]. Como feito em [9], no topo de cada dígito manuscrito, exibimos seu identificador, seguido pela correta classificação e pelo resultado incorreto produzido pelo nosso algoritmo. 


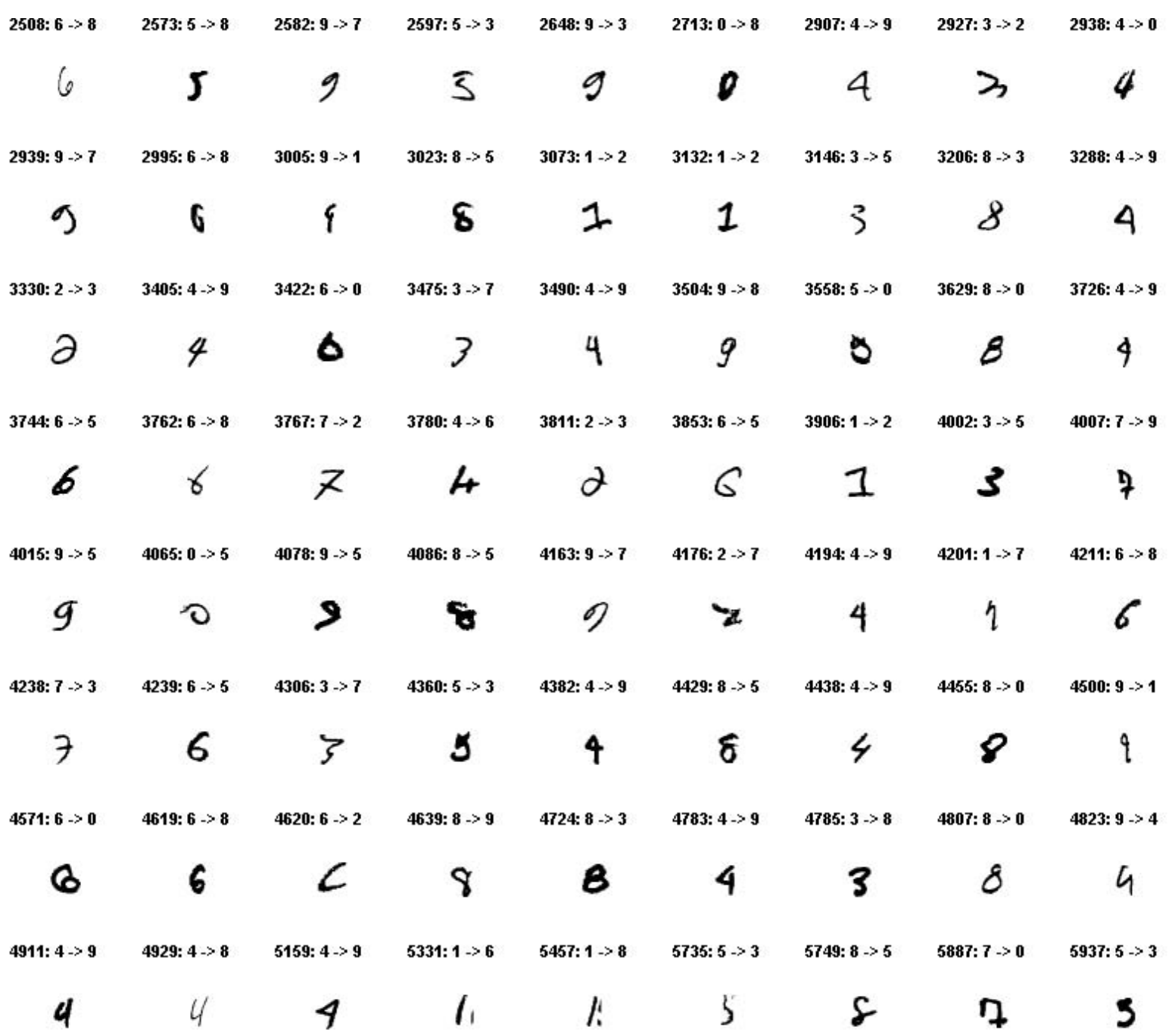

Figura 3.20: Classificações incorretas produzidas pelo nosso classificador 5-NN, usando o banco de imagens MNIST [52]. Como feito em [9], no topo de cada dígito manuscrito, exibimos seu identificador, seguido pela correta classificação e pelo resultado incorreto produzido pelo nosso algoritmo. 


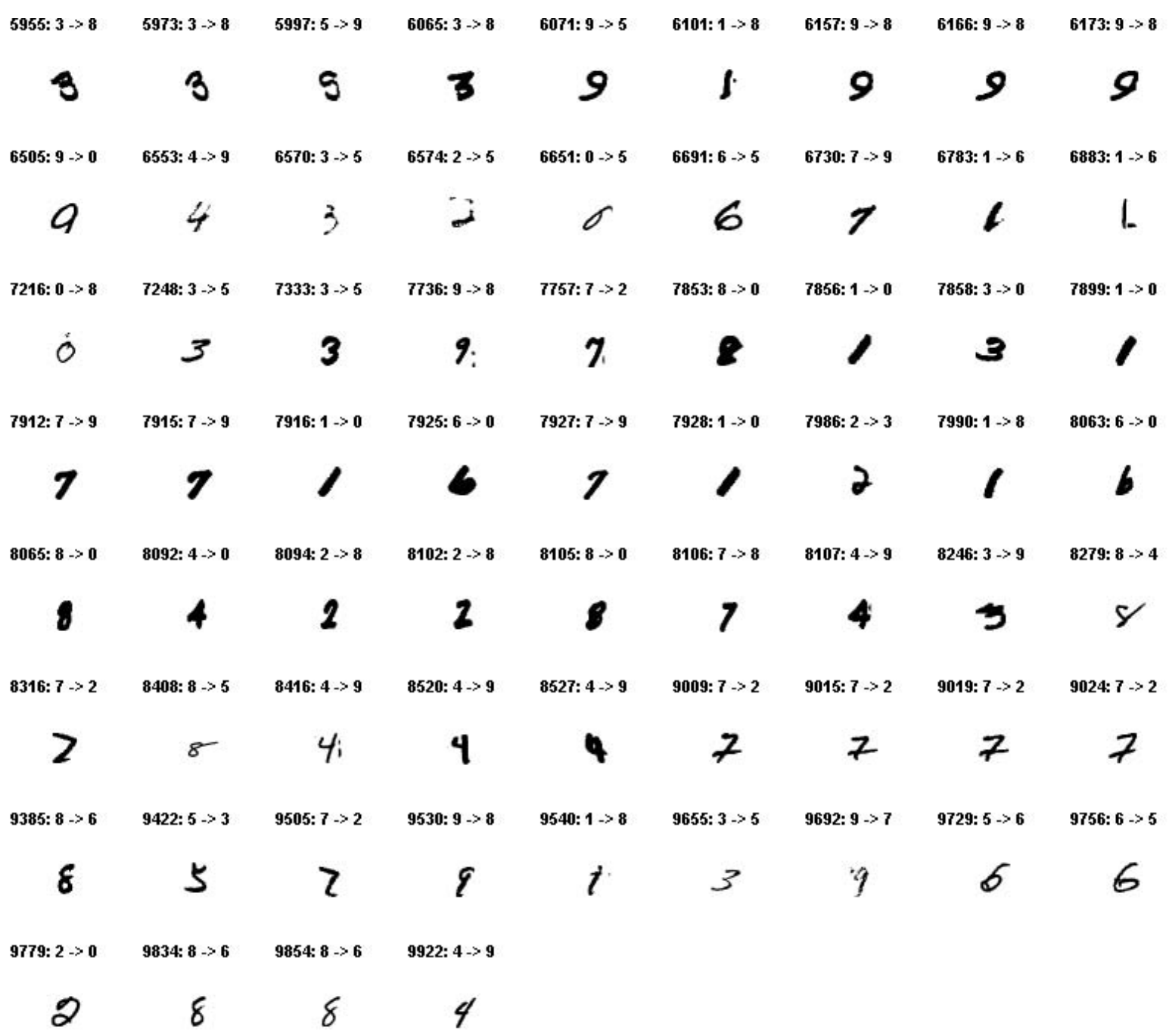

Figura 3.21: Classificações incorretas produzidas pelo nosso classificador 5-NN, usando o banco de imagens MNIST [52]. Como feito em [9], no topo de cada dígito manuscrito, exibimos seu identificador, seguido pela correta classificação e pelo resultado incorreto produzido pelo nosso algoritmo. 
entre 0.0039 e 0.12 . Por exemplo, usando nosso classificador 5-NN com todos os 60000 protótipos, a taxa de erro obtida foi de 0.0211 , menor que a taxa de 0.0309 obtida pelo $K$ NN usando distância $L_{2}$, demonstrando a importância das relações espaciais para melhorar o resultado de classificação. Todos os 211 erros são exibidos nas Figuras 3.19 a 3.21. A Figura 3.18 ilustra o comportamento do nosso classificador $K$-NN para diferentes valores de $K$, resultando em taxas relativamente baixas de erro mesmo para valores altos de $K$, indicando robustez das classificações geradas por nosso método.

\subsection{Aplicação 2: colorização assistida por computa- dor}

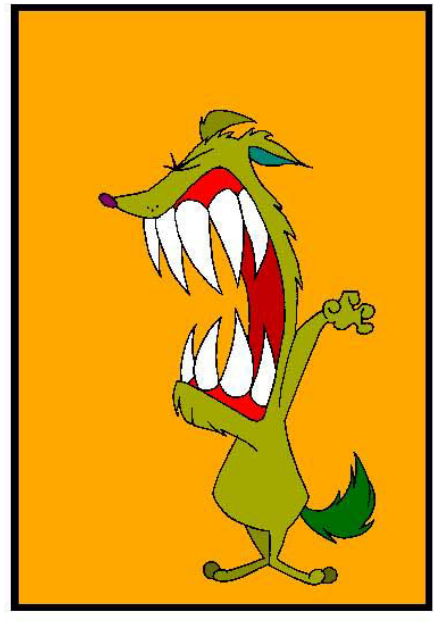

(Modelo)

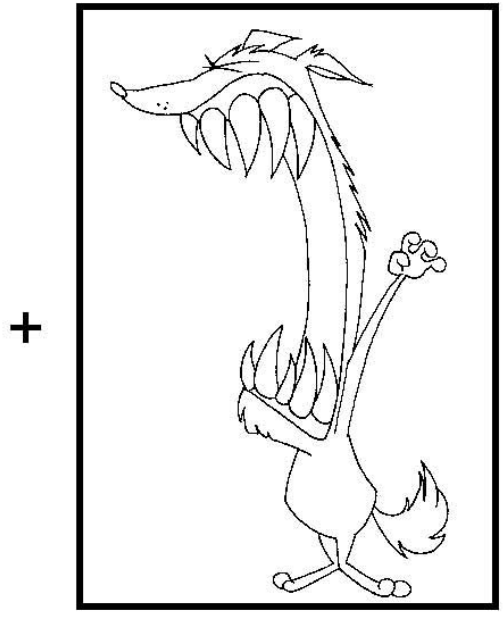

(Entrada)

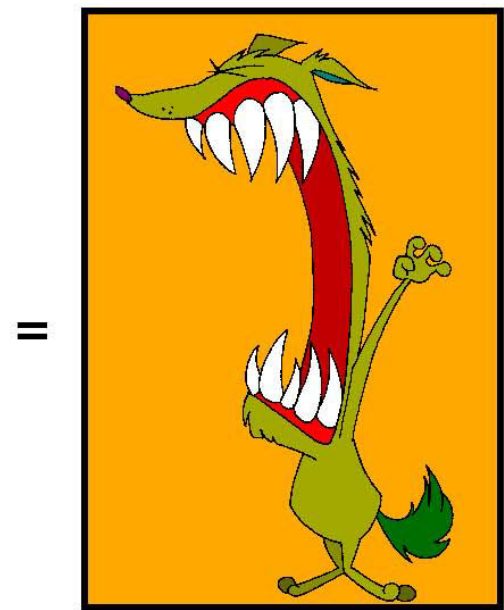

(Resultado)

Figura 3.22: Resumo da colorização assistida por computador.

Desde o final da década de 1960 e início da década de 1970, várias técnicas foram propostas para incluir o uso do computador na produção de animações tradicionais 2D, produzidas quadro-a-quadro. Por exemplo, métodos usando quadros-chave para automatizar a geração de quadros intermediários por interpolação foram descritos em [6, 19].

Conforme observado em [14, 15], o desenho animado assistido por computador é uma das áreas mais desafiadoras no campo de animação por computador. Desde o trabalho 
seminal de Ed Catmull [25], detalhando os diferentes estágios de produção e ressaltando os desafios envolvidos em animações 2D, intensas pesquisas têm sido desenvolvidas nessa área. Entretanto, muitos problemas se mantêm sem solução devido a diversas dificuldades. Por um lado, desenhos animados tradicionais dependem fortemente de uma interpretação artística da realidade. Por outro, animação 2D depende da dinâmica em um mundo imaginário 3D, desenhada por traços 2D pelo artista. Estes aspectos trasformam a maioria das tarefas de animações por computador em problemas ambíguos, fortemente dependentes de questões subjetivas relacionadas a percepção artística.

$\mathrm{Na}$ animação tradicional, geralmente, cada quadro apresenta pequenas alterações em relação ao quadro anterior, de modo a transmitir um movimento suave. Um dos estágios mais trabalhosos e tediantes na produção de uma animação 2D é a colorização de cada quadro. Com o objetivo de agilizar este processo, o problema de colorização assistida por computador (CAC) $[67]^{3}$ pode ser definido da seguinte maneira. Dada uma sequência de animação, o objetivo é rastrear elementos estruturais 2D, representando regiões, ao longo da sequência para estabelecer correspondências entre as regiões provenientes de quadros consecutivos [14], possibilitando uma propagação automática de cores para diferentes quadros. Isto permite modificações rápidas nas cores de uma sequência completa de animação, através de edições/renderizações de alguns quadros isolados.

Um resumo do problema é desenhado na Figura 3.22. Para uma descrição mais aprofundada sobre colorização assistida por computador, veja [15].

\subsubsection{Histórico}

O processo de casamento entre as regiões presentes em quadros consecutivos é uma tarefa não trivial devido à liberdade do artista em produzir a dinâmica dos movimentos na sequência de animação. Existem vários métodos de colorização baseados em correspondência entre regiões, por exemplo [58, 73, 79].

As informações de localização, forma e topologia das regiões são popularmente usadas para o cálculo das correspondências [15] entre os quadros consecutivos, principalmente

\footnotetext{
${ }^{3}$ Esta parte foi desenvolvida em conjunto com o professor Luiz Velho, responsável por nos trazer a aplicação de colorização e disponibilizar as sequências de animações usadas nos experimentos, incluindo as três animações fornecidas por Cesar Coelho.
} 
na presença de pequenas alterações representando movimentos suaves na animação. Por exemplo, como característica extraída das imagens, além da informação da posição, tipicamente usa-se informações de volume, geometria e relações de vizinhança entre as regiões, definidas pelos traços do desenhista.

Um importante trabalho desenvolvido para o problema CAC foi apresentado por Bezerra et al. [14], descrevendo um método baseado em três fatores: área de regiões, pontos 2D (posição) e diferenças topológicas entre regiões adjacentes. Os autores definem uma métrica chamada degree of topological difference $D T D(r, s)$, mensurando a distância entre duas regiões, $r$ e $s$, comparando as respectivas funções de adjacência, definidas para cada região, representando de maneira sofisticada a morfologia das regiões circundantes.

Neste trabalho, propomos uma abordagem mais simples, baseada em área, perímetro e pontos 2D, usados para explorar as relações espaciais através do termo de Markov, definido na Equação 3.3.1. Resultados encorajadores são apresentados na Seção 3.5.4.

\subsubsection{Abordagem de colorização}

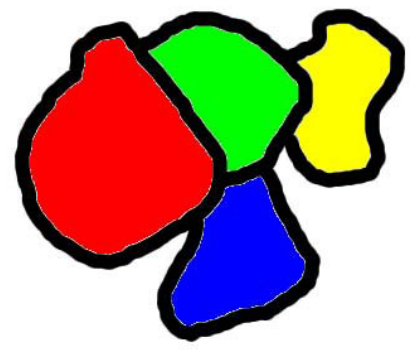

(Regioes)

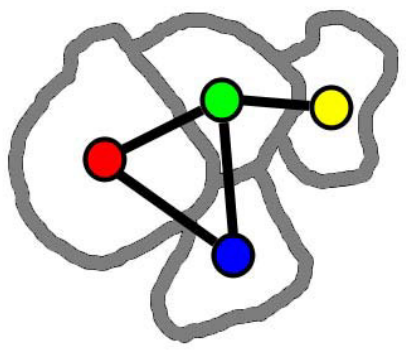

(Grafo)

Figura 3.23: Arestas são criadas entre regiões adjacentes.

Para encontrar uma correspondência entre as regiões, cada região é representada pelo seu centróide. Dados dois quadros da animação, um colorido e outro não-colorido, o objetivo é encontrar uma correspondência entre padrões de pontos, um representando o colorido e outro representando o não-colorido. Os padrões de pontos são codificados por grafos. O quadro colorido é representado pelo grafo modelo $G_{m}$, enquanto o quadro 
não-colorido é representado pelo grafo de entrada $G_{i}$.

Os dois grafos são obtidos de maneira similar (Figura 3.23). Cada centróide é representado por um vértice. Arestas são criadas entre as regiões adjacentes, assumindo que as relações de adjacência são geralmente mantidas entre um quadro e outro. Ambos grafos são planares, resultando em uma computação eficiente das mensagens BP, conforme descrito na Seção 3.3.2. O objetivo é encontrar um MCS entre os grafos da entrada e o modelo para propagar as cores do quadro colorido para o não-colorido (Figura 3.22).

\subsubsection{Distância entre regiões}

Para cada vértice $v$, as informações sobre a aparência são associadas ao atributo $\mu(v)$, codificando a área e o perímetro da região representada por $v$. No papel do termo linear da Equação 3.13, usamos:

$$
D_{p}\left(f_{p}\right)=\max \left\{\frac{\left|\mu_{A}(p)-\mu_{A}\left(f_{p}\right)\right|}{\mu_{A}(p)}, \frac{\left|\mu_{C}(p)-\mu_{C}\left(f_{p}\right)\right|}{\mu_{C}(p)}\right\}
$$

onde $\mu_{A}$ e $\mu_{C}$ representam a área e o perímetro (ou o comprimento do contorno da região), respectivamente, $p \in V_{i}$ e $f_{p} \in V_{m}$. Para mapear um vértice de entrada $p$ a um vértice modelo $f_{p}$, os dois atributos (área e perímetro) devem ser satisfeitos simultâneamente de maneira a deixar a regiões ambíguas para o usuário colorir manualmente.

\subsubsection{Experimentos}

Em todos os exemplos, as regiões brancas representam aquelas que não foram classificadas ou coloridas pelo método. A Figura 3.24 ilustra uma comparação com o método de Bezerra et al., descrito em [14]. Neste exemplo, todas as regiões coloridas foram corretamente classificadas pelo nosso método, deixando 6 regiões brancas, dentre as quais, 5 representam 'regiões esquecidas' representando regiões da entrada com correspodência no modelo (e que deveriam ser colorizadas pelo algoritmo), e 1 região corretamente não-classificada, representando uma nova região que surgiu acima da língua. O método de Bezerra et al. [14] produziu 8 'regiões esquecidas', ilustrando uma considerável melhoria por parte de nosso algoritmo. Note que dentre as 5 'regiões esquecidas' produzidas pela nossa abordagem, 4 foram resultantes de mudanças nas relações de adjacência entre as regiões, 


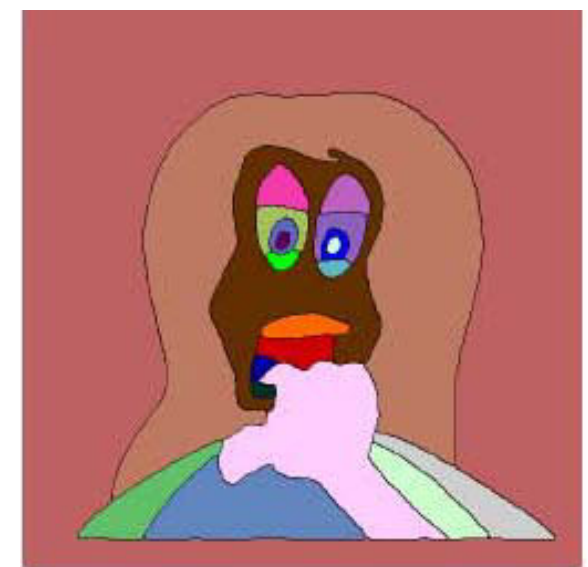

(Modelo)

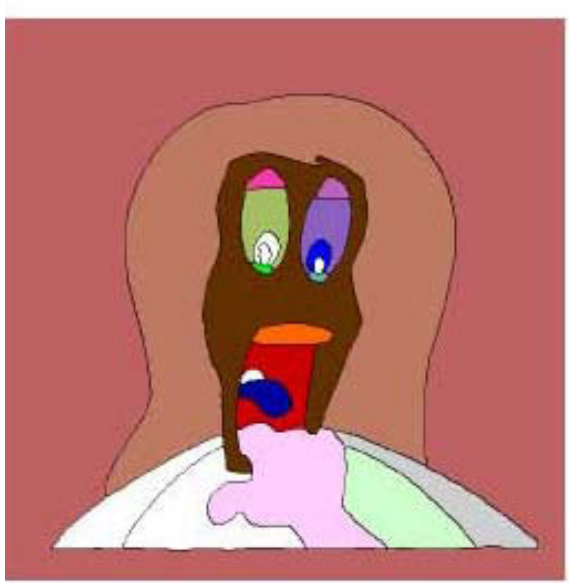

(Nosso resultado)

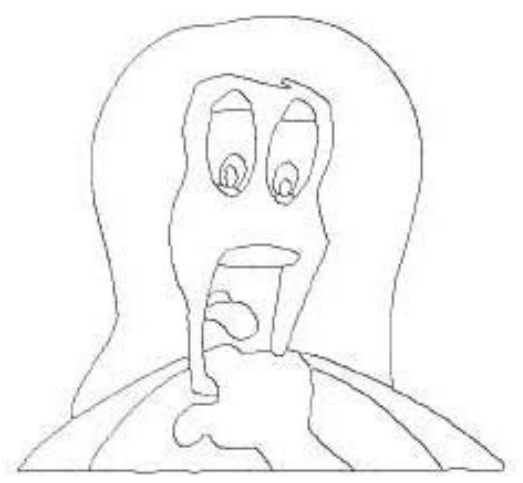

(Entrada)

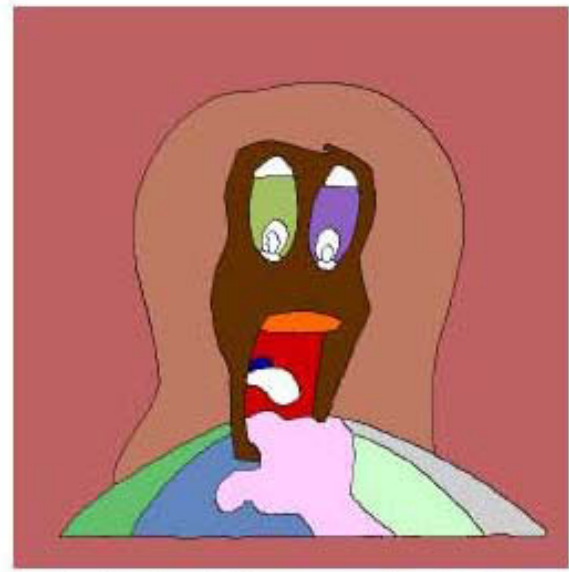

(Trabalho anterior)

Figura 3.24: Exemplo 'face'. Partindo do quadro colorido, representado pelo grafo modelo. O objetivo é colorir o próximo quadro (não-colorido), representado pelo grafo de entrada. Os resultados, do nosso método e do método de Bezerra et al. [14], são exibidos para comparação. 


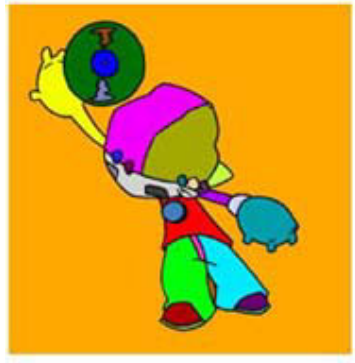

(Modelo)

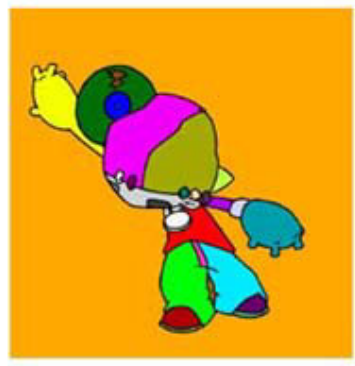

(Resultado)

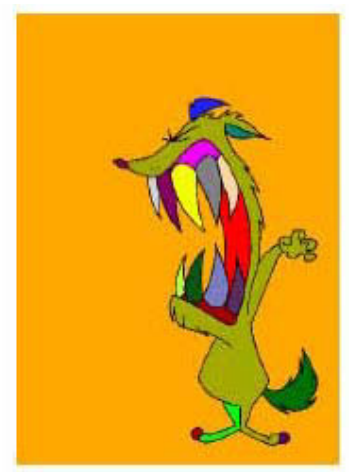

(Modelo)

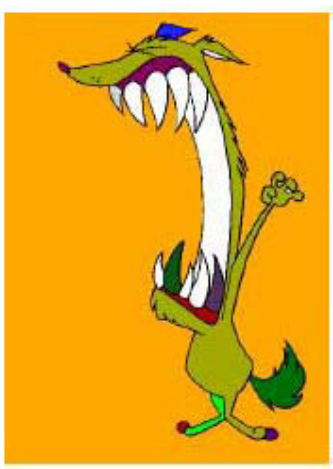

(Resultado)

(a)

(b)

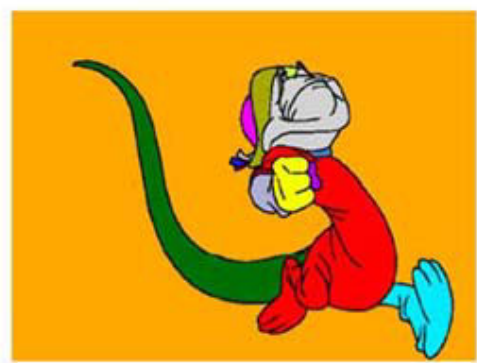

(Modelo)

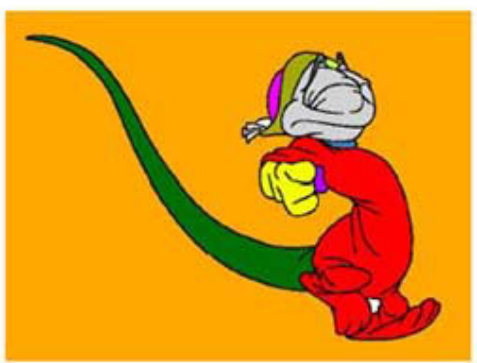

(Resultado)

(c)

Figura 3.25: Exemplos de colorização das animações (a) 'cufa', (b) 'lobinho' e (c) 'calango'. Para cada exemplo, apresentamos o modelo e a entrada colorizada pelo nosso método, respectivamente. 
penalizadas pela nossa técnica. As mudanças de adjacência ocorrem nas duas regiões do corpo e nas duas pupilas.

Nosso método foi testado com mais três animações: 'cufa', 'calango' e 'lobinho'4. Os resultados são ilustrados nas Figuras 3.26 a 3.30. Neste experimento, testamos três fatores: deformações na aparência devido a grandes variações nas regiões correspondentes em termos de área e de perímetro, e deformações estruturais causadas pela dinâmica da animação, provocando fusões e divisões de regiões.

Por exemplo, na Figura 3.25(a), ocorre uma oclusão parcial no disco, causando o desaparecimento de uma região (um dos brilhos no disco), além de causar uma grande variação em sua área visível. A Figura 3.25(b) ilustra um exemplo desafiador, com fortes deformações tanto nas informações de aparência (área e perímetro) quanto nas informações estruturais (devido ao aparecimento de novos dentes, alterando as relações de adjacência). Na Figura 3.25(c), ocorre o desaparecimento de algumas regiões devido à fusão das regiões entre perna/corpo e braço/mão, e o aparecimento de uma nova região entre o pé e a perna.

Finalmente, resultados quantitativos relativos às Figuras 3.24 a 3.30, são ilustrados na Tabela 3.1, exibindo o nome da animação, os números dos quadros, as quantidades de vértices em cada grafo $\left(\left|V_{m}\right|\right.$ e $\left.\left|V_{i}\right|\right)$, a quantidade de regiões coloridas incorretamente, o número de regiões esquecidas em branco mas com correspondência colorida no modelo, e o total de novas regiões deixadas corretamente em branco (sem classificação).

\footnotetext{
${ }^{4}$ As imagens relativas às três animações ('cufa', 'calango' e 'lobinho') foram gentilmente cedidas por Cesar Coelho.
} 

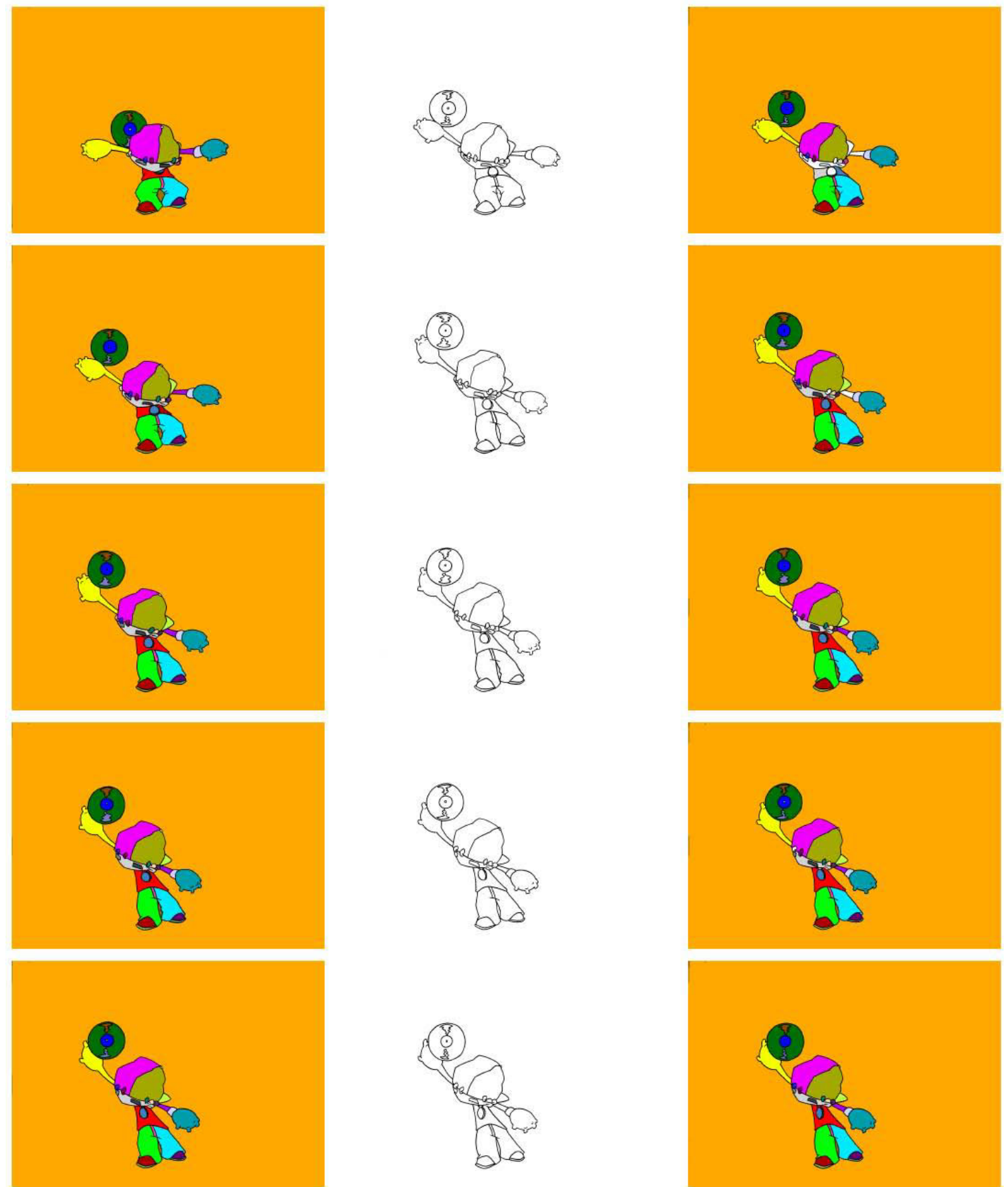

(Modelo)

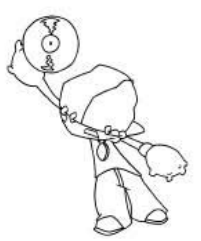

(Entrada)

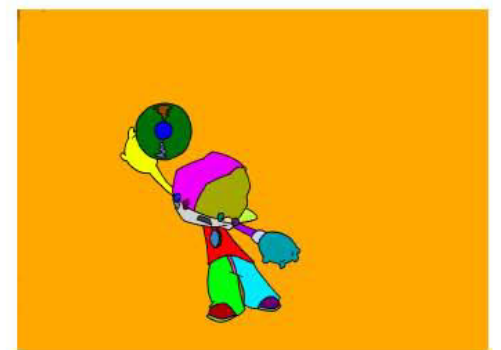

(Nosso resultado)

Figura 3.26: Exemplos de colorização da animação 'cufa'. Quadros 35 a 40. 

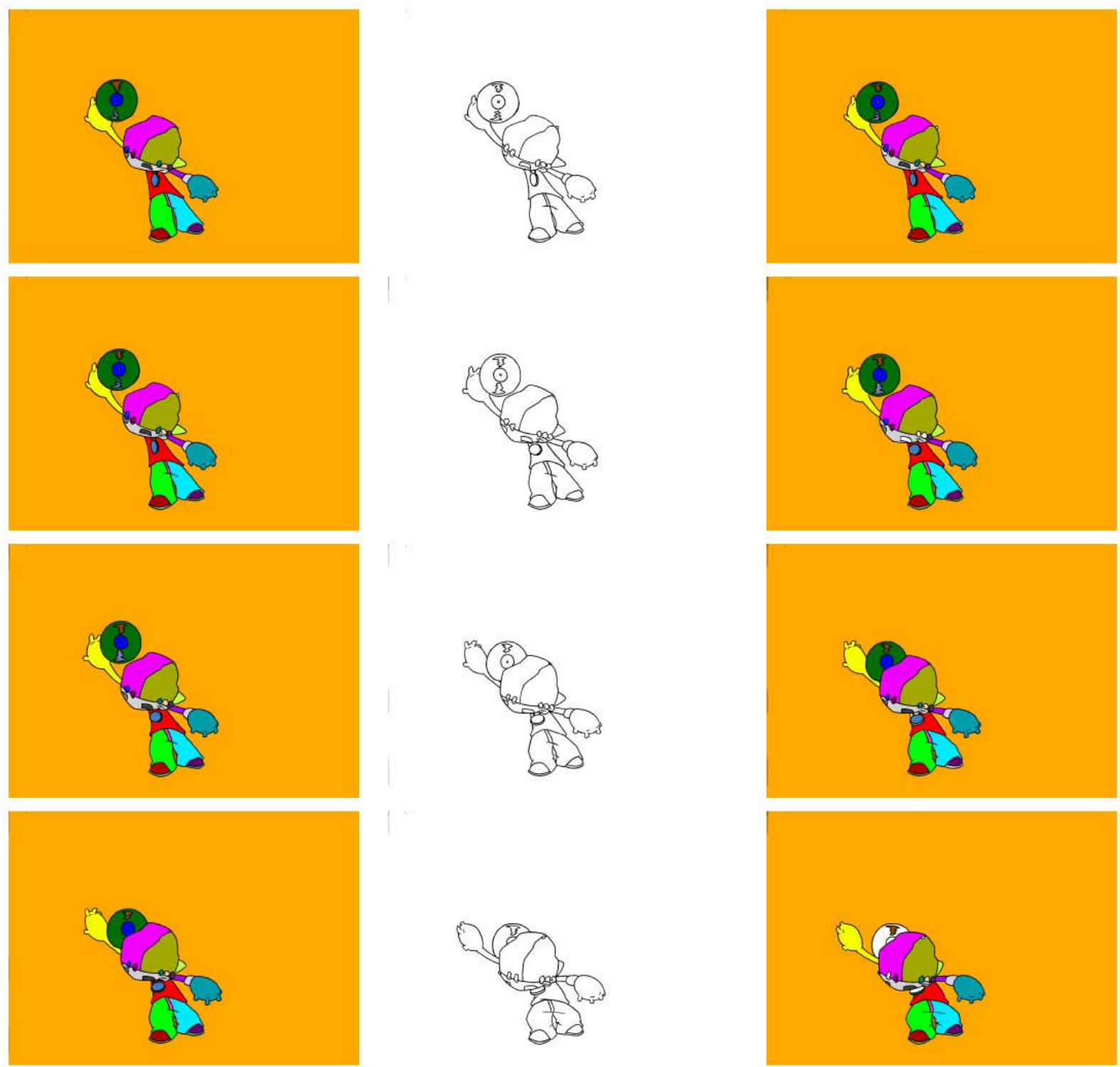

(Modelo)

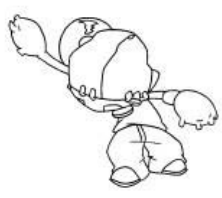

(Entrada)

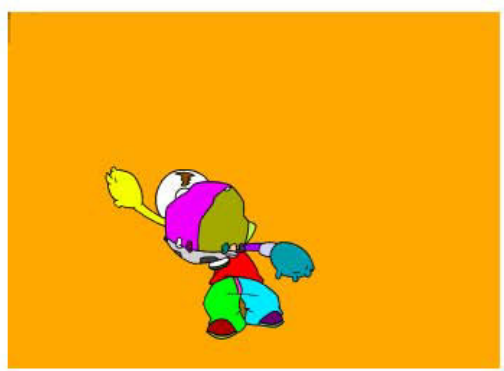

(Nosso resultado)

Figura 3.27: Exemplos de colorização da animação 'cufa'. Quadros 40 a 44. 

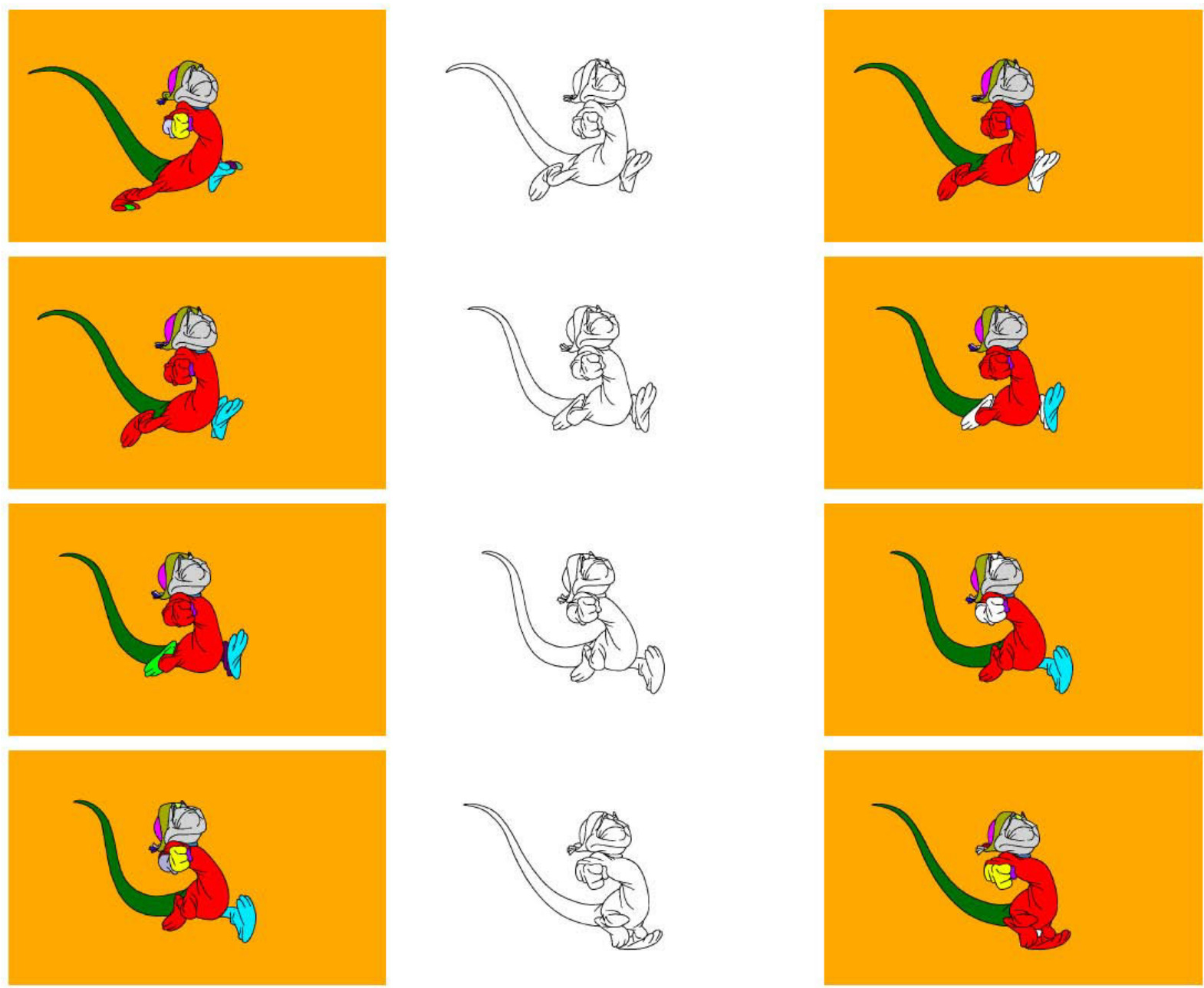

(Modelo)

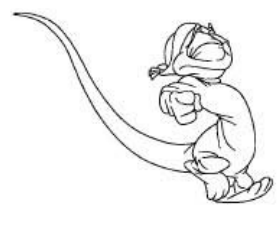

(Entrada)

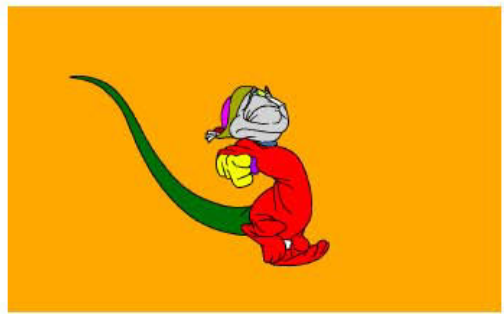

(Nosso resultado)

Figura 3.28: Exemplos de colorização da animação 'calango'. Quadros 21 a 25. 

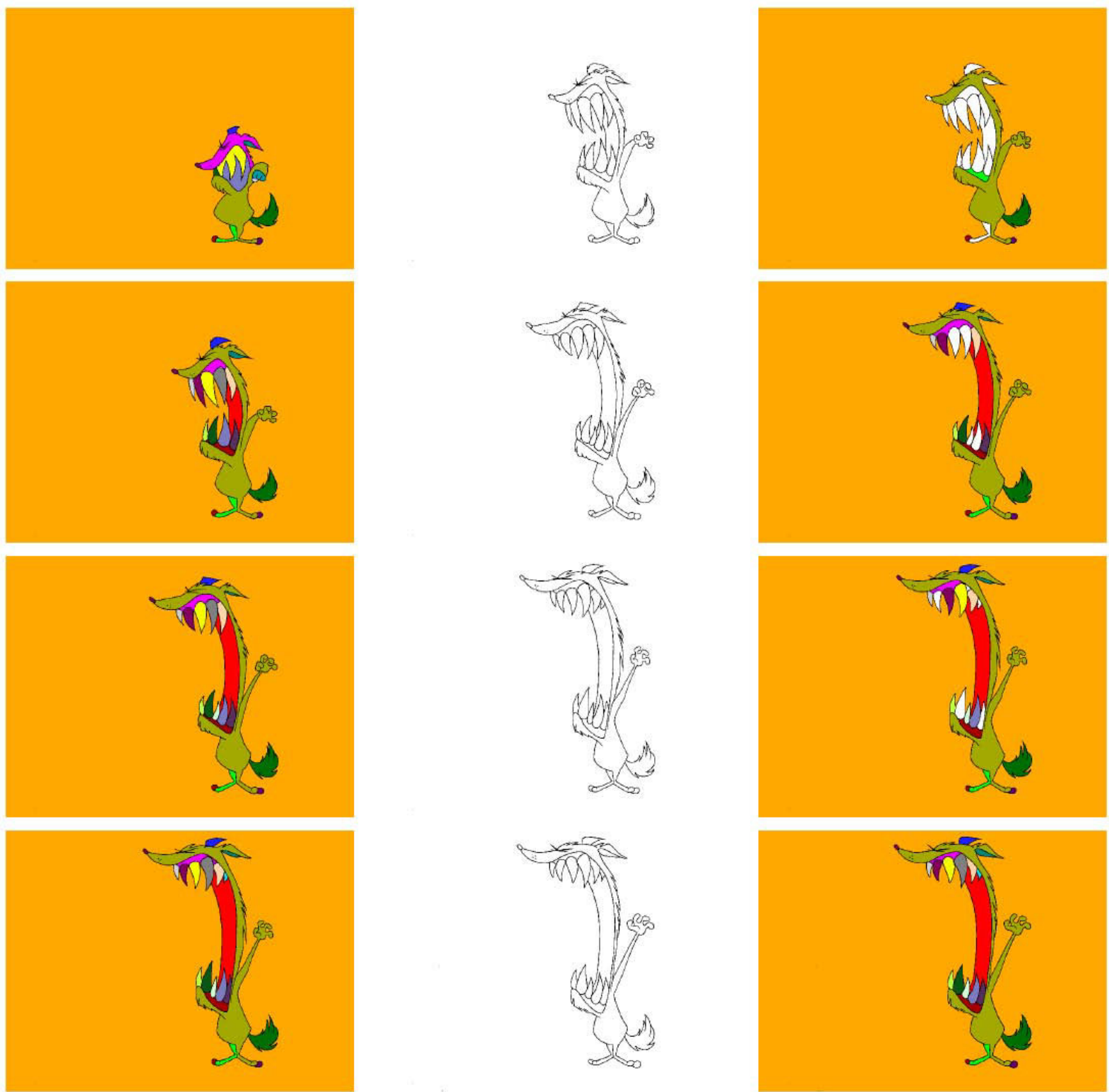

(Modelo)

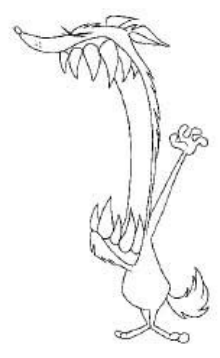

(Entrada)

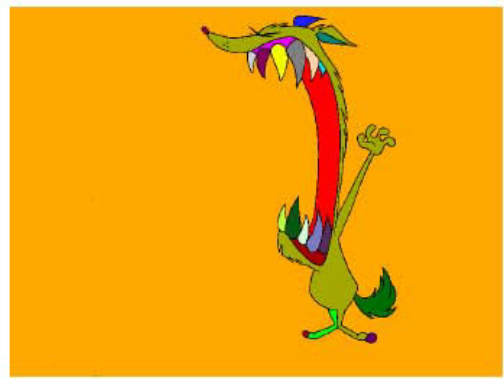

(Nosso resultado)

Figura 3.29: Exemplos de colorização da animação 'lobinho'. Quadros 25 a 29. 

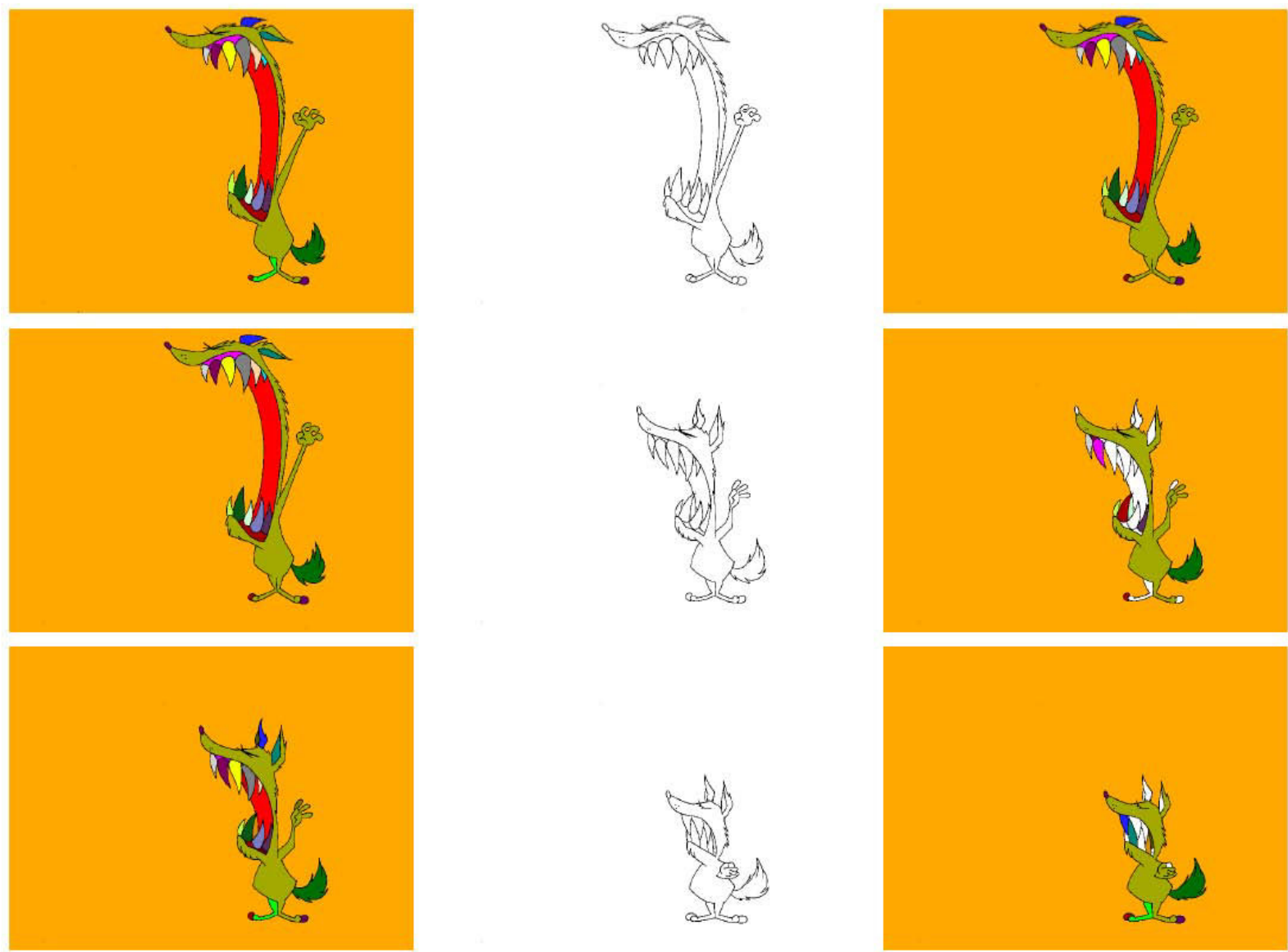

(Modelo)

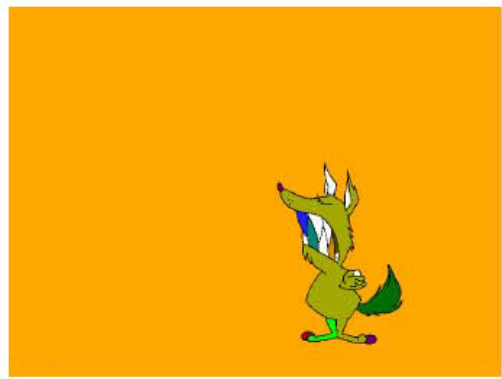

(Nosso resultado)

(Entrada)

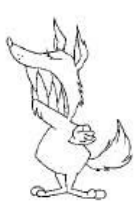

Figura 3.30: Exemplos de colorização da animação 'lobinho'. Quadros 29 a 32. 
Tabela 3.1: Resultados quantitativos das Figuras 3.24 a 3.30.

\begin{tabular}{|c|c|c|c|c|c|c|}
\hline animação & quadros & $\left|V_{m}\right|$ & $\left|V_{i}\right|$ & $\begin{array}{c}\text { colorizações } \\
\text { incorretas }\end{array}$ & $\begin{array}{c}\text { 'regiões } \\
\text { esquecidas' }\end{array}$ & $\begin{array}{c}\text { corretamente } \\
\text { não-classificados }\end{array}$ \\
\hline face & - & 18 & 22 & 0 & $5(22.72 \%)$ & $1(100 \%)$ \\
cufa & 35,36 & 37 & 35 & $4(11.43 \%)$ & $8(22.86 \%)$ & 0 \\
cufa & 36,37 & 35 & 36 & $1(2.78 \%)$ & $3(8.33 \%)$ & $2(100 \%)$ \\
cufa & 37,38 & 37 & 36 & 0 & $3(8.33 \%)$ & 0 \\
cufa & 38,39 & 36 & 36 & $1(2.78 \%)$ & 0 & $1(100 \%)$ \\
cufa & 39,40 & 36 & 35 & 0 & 0 & 0 \\
cufa & 40,41 & 35 & 36 & 0 & $2(5.56 \%)$ & $1(100 \%)$ \\
cufa & 41,42 & 36 & 35 & 0 & $4(11.43 \%)$ & 0 \\
cufa & 42,43 & 35 & 36 & 0 & $4(11.11 \%)$ & $2(100 \%)$ \\
cufa & 43,44 & 36 & 32 & $1(3.13 \%)$ & $4(12.50 \%)$ & 0 \\
calango & 21,22 & 25 & 17 & 0 & $1(5.88 \%)$ & 0 \\
calango & 22,23 & 17 & 20 & 0 & 0 & $3(100 \%)$ \\
calango & 23,24 & 20 & 23 & 0 & 0 & $5(100 \%)$ \\
calango & 24,25 & 23 & 21 & $1(4.76 \%)$ & $3(14.28 \%)$ & $1(100 \%)$ \\
lobinho & 25,26 & 15 & 23 & $1(4.35 \%)$ & $5(21.74 \%)$ & $10(100 \%)$ \\
lobinho & 26,27 & 23 & 23 & $1(4.35 \%)$ & $8(34.78 \%)$ & $2(100 \%)$ \\
lobinho & 27,28 & 23 & 24 & $2(8.33 \%)$ & $3(12.50 \%)$ & $2(100 \%)$ \\
lobinho & 28,29 & 24 & 24 & 0 & 0 & 0 \\
lobinho & 29,30 & 24 & 23 & 0 & $1(4.35 \%)$ & 0 \\
lobinho & 30,31 & 23 & 29 & $4(13.79 \%)$ & $10(34.48 \%)$ & $6(75 \%)$ \\
lobinho & 31,32 & 29 & 21 & $4(19.05 \%)$ & $7(33.33 \%)$ & $2(100 \%)$ \\
\hline
\end{tabular}




\subsection{Resumo da abordagem BP}

Em nossa segunda contribuição, exploramos as relações espaciais propondo um novo termo de Markov, representando penalidades geométricas em termos de distância, orientação e adjacência. O problema de casamento de padrões de pontos foi formulado por campos aleatórios de Markov (MRFs), onde estendemos o algoritmo eficiente baseado em propagação de crenças (BP), descrito em [39], para explorar o novo termo de Markov e estimar uma solução. A eficiência foi obtida através do uso de grafos esparsos.

Para o casamento entre formas (SM), propomos uma nova representação, simples e compacta, explorando as relações espaciais entre os pontos amostrados nas bordas do objeto, resultando em aproximações poligonais para silhueta e para a textura. Nosso experimentos ilustraram resultados satisfatórios de reconhecimento de objetos 3D e de dígitos manuscritos.

Para o problema de colorização assistida por computador (CAC), mostramos resultados encorajadores, testando nossa abordagem para deformações de aparência e de estrutura, usando diferentes animações. Através de fusão e de divisão de regiões, avaliamos a qualidade do método BP para casar grafos com incompatibilidades topológicas. 


\section{Capítulo 4}

\section{Conclusões}

\subsection{Comentários finais}

Duas abordagens para casamento de padrões de pontos, baseadas em casamento entre grafos relacionais com atributos, foram propostas como contribuições originais desta Tese. A primeira executa buscas discretas baseadas em grafos deformados (DG). A segunda representa um método de relaxação probabilística baseado em propagação de crenças (BP). Ambas exploram informações estruturais representadas pelas relações espaciais entre os pontos e informações de aparência, específicos para cada aplicação, para facilitar o processo de casamento.

A Tabela 4.1 resume os resultados obtidos das duas contribuições sobre as quatro aplicações tratadas neste trabalho. Os melhores resultados, usando o método DG, foram para os problemas 2DEM e INIS, enquanto que os melhores resultados obtidos até o momento, usando o método BP, foram para os problemas SM e CAC.

Tabela 4.1: Resumo do desempenho das duas contribuições, DG e BP, sobre as quatro aplicações tratadas neste trabalho.

\begin{tabular}{|c|c|c|c|c|}
\hline & 2DEM & INIS & SM & CAC \\
\hline DG & Bom & Bom & Ruim & $?$ \\
\hline BP & Ruim & Ruim & Bom & Médio \\
\hline
\end{tabular}




\section{Limitações das duas abordagens}

O método BP usual não é adequado para grafos com múltiplos loops e, em geral, não produz boas aproximações para os problemas 2DEM e INIS, onde os conjuntos de pontos podem variar de centenas a milhares de pontos, produzindo um número muito grande de loops nos dois grafos sendo casados.

Entretanto, para o problema CAC, apesar do grafo de entrada e do grafo modelo possuírem múltiplos loops, este número é bastante reduzido, devido à limitação do número de regiões presentes nas animações testadas, justificando os resultados promissores, porém limitados (veja mais adiante os comentários adicionais sobre a abordagem BP), obtidos durante os experimentos.

Para o problema SM, as representações esparsas usadas para codificar as aproximações poligonais do contorno dos objetos contém um número muito reduzido de arestas, resultando em informações estruturais insuficientes para o método DG, sendo esta abordagem inadequada para este tipo de aplicação. Para o problema CAC, o método DG não foi testado neste trabalho, sendo uma sugestão para um passo futuro.

\section{Comentários adicionais sobre a abordagem DG}

Nossa abordagem baseada em DGs é extremamente rápida, com uma complexidade de tempo $O\left(n^{2}\right)$, onde $n=\max \left\{\left|V_{i}\right|,\left|V_{m}\right|\right\}$. Por exemplo, o algoritmo mais rápido conhecido para o problema de casamento entre grafos bipartidos (BGM) possui complexidade computacional $O\left(n^{\frac{5}{2}}\right)$ [69]. O algoritmo graduated assigment (GA) possui complexidade $O\left(n^{3}\right)$ [29]. Tanto o método Húngaro para BGM quanto GA foram testados para o problema 2DEM, onde foram superados pelo método DG em termos de qualidade de resultados.

Além de superar outros métodos em termos de qualidade e rapidez, a simplicidade de nosso método permite que implementações sejam feitas rapidamente, sendo facilmente integrável a um sistema gráfico permitindo interações com o usuário. Nas duas aplicações, 2DEM e INIS, o método DG produziu resultados de alta qualidade, explorando de maneira eficiente o conhecimento a priori fornecido pelo usuário. 


\section{Comentários adicionais sobre a abordagem BP}

Exceto por alguns poucos tipos simples de grafos, por exemplo ávores ou grafos com um único loop, ainda é necessário um entendimento teórico mais profundo sobre os algoritmos baseados em BP para grafos com loops, conforme observado em [94, 95]. Quando aplicado a árvores, o método BP de máximo-produto é conhecido na literatura por convergir a um ponto fixo. Já para grafos com um único loop, o mesmo algoritmo converge para um ponto fixo estável ou oscila de maneira periódica.

Para o problema SM, nossa abordagem baseada em BP produziu resultados satisfatórios devido ao uso de representações esparsas para as formas, codificadas através de grafos esparsos, cujas componentes conexas foram, de maneira geral, árvores ou grafos com um único loop, permitindo aproximações eficientes em termos de qualidade e tempo de processamento. A nova representação é mais simples e compacta que, por exemplo, a representação baseada em distâncias internas, proposta em [56], usada na abordagem descrita em [27], combinando aprendizado e casamento.

Para o problema CAC, apesar dos resultados encorajadores, a qualidade dos resultados de colorização dependeram de ajustes finos do parâmetro $\lambda_{1}$ na Equação 3.13. Nos experimentos, as arestas foram criadas de acordo com a adjacência entre as regiões do desenho, produzindo uma variedade de pequenos loops. No caso de grafos com mais de um loop, as mensagens podem circular indefinidamente e o algoritmo baseado em BP pode não convergir, conforme descrito em [70].

\subsection{Trabalhos futuros}

Recentemente, Caetano et al. [22] propuseram um método de aprendizado para o problema SM, usando pares de treinamento, cujas formas são manualmente casadas, de maneira a ajustar os dois termos da equação de atribuição quadrática, de acordo com os mapeamentos esperados. Os autores exibiram importantes resultados, mostrando que algoritmos de atribuição linear, como é o caso do método Húngaro, combinados com este esquema de aprendizado, podem produzir resultados comparáveis aos melhores métodos de relaxação disponíveis.

Para melhorar a classificação, por exemplo, para o problema SM, é interessante investi- 
gar a possibilidade de combinar o nosso método BP com um esquema de aprendizado, por exemplo [27], substituindo as representações baseadas em distâncias internas [56] pelas representações esparsas propostas nesta Tese.

Para melhorar os resultados do problema CAC, é interessante testar o nosso algoritmo DG com ICP. Outra possibilidade é testar usando a abordagem generalized belief propagation (GBP), descrita em [97]. Nas abordagens BP típicas, as mensagens são sempre provenientes de um único vértice para outro vértice. A idéia básica por trás do GBP é propagar mensagens de grupos de vértices para outros grupos de vértices, fornecendo informações mais significativas de contexto para obter uma melhor estimação da solução. Em [97], os autores de afirmam que o GBP pode melhorar significativamente o método usual de BP no caso de grafos com pequenos loops.

Finalmente, é valioso estender o domínio das aplicações, especialmente envolvendo os dois métodos propostos neste trabalho. Por exemplo, devido ao fato do nosso algoritmo BP ser baseado em uma formulação MRF representando um 'mapeamento da cena para o modelo' (Seção 3.1.2), isto permite procurar por múltiplas ocorrências do modelo, imersas na cena. Usando esta idéia, podemos estender o problema de segmentação interativa de imagens para segmentar múltiplos objetos. Na Figura 4.1, ilustramos alguns resultados preliminares, usando nossa abordagem BP para computar homomorfismos, representando segmentações das diferentes partes de múltiplos objetos. 

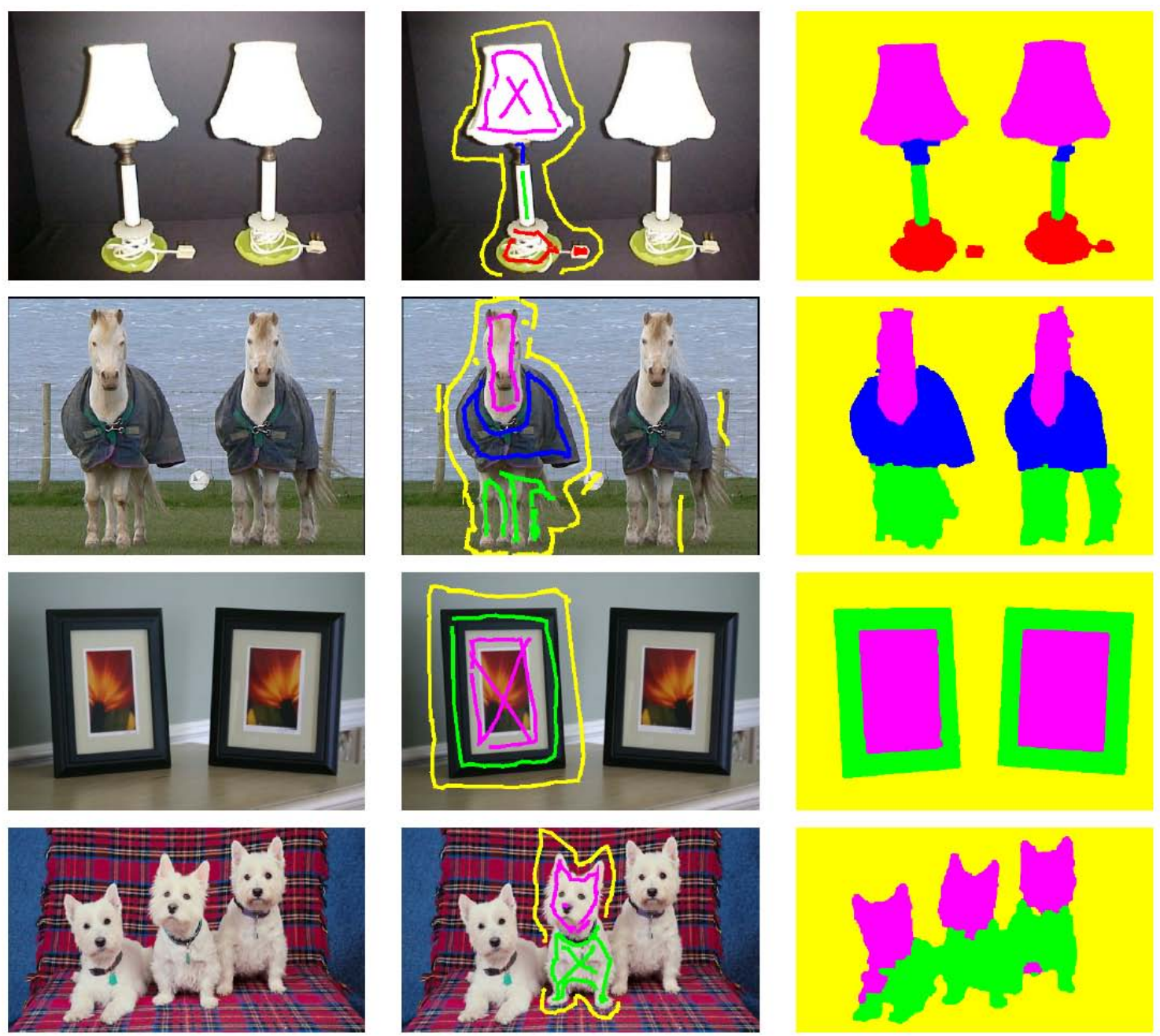

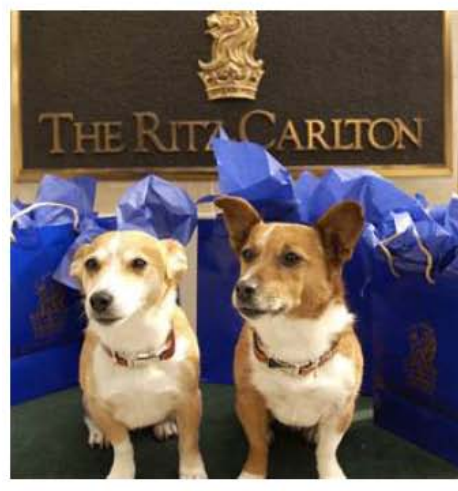

(a)

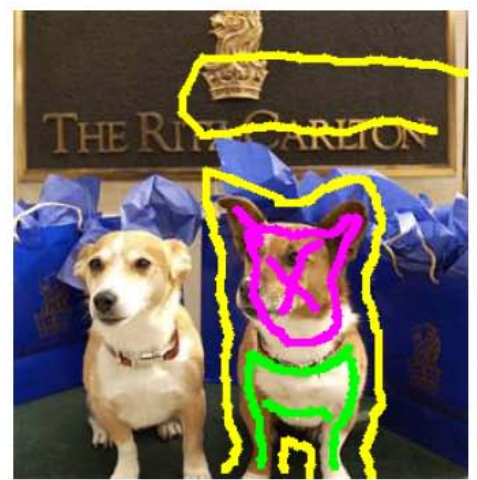

(b)

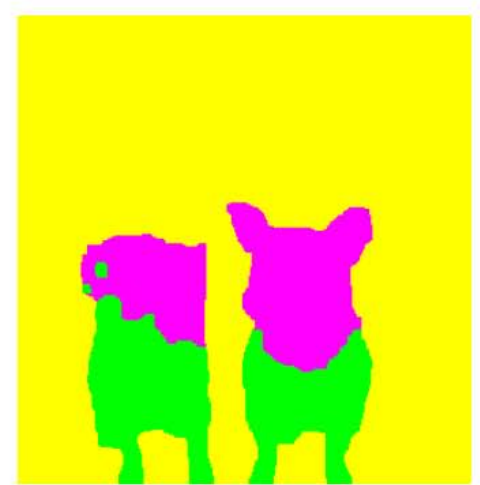

(c)

Figura 4.1: (a) Imagem de entrada. (b) Traços do usuário sobrepostos na imagem. (c) Segmentação. 


\section{Referências Bibliográficas}

[1] http://structuralsegm.sourceforge.net/.

[2] http://www.lecb.ncifcrf.gov/2dgeldatasets/.

[3] R. Adams e L. Bischof, Seeded region growing, IEEE Trans. Pattern Anal. Mach. Intell. 16 (1994), no. 6, 641-647.

[4] A. Almansa, M. Gerschuni, A. Pardo e J. Preciozzi, Processing of 2d electrophoresis gels, 2007 ICCV International Workshop on Computer Vision for Developing Regions, October 2007.

[5] D. Anguelov, D. Koller, P. Srinivasan, S. Thrun, H.-C. Pang e J. Davis, The correlated correspondence algorithm for unsupervised registration of nonrigid surfaces, Advances in Neural Information Processing Systems (Vancouver, Canada), 2004.

[6] R. M. Baecker, Picture-driven animation, AFIPS '69 (Spring): Proceedings of the May 1416, 1969, spring joint computer conference (New York, NY, USA), ACM, 1969, pp. 273-288.

[7] X. Bai e G. Sapiro, Geodesic matting: a framework for fast interactive image and video segmentation and matting, Int. J. Comput. Vision 82 (2009), no. 2, 113-132.

[8] H. G. Barrow e R. J. Popplestone, Relational descriptions in picture processing, Machine Intelligence VI (1971), 377-396.

[9] S. Belongie, J. Malik e J. Puzicha, Shape matching and object recognition using shape contexts, IEEE Trans. Pattern Anal. Mach. Intell. 24 (2002), no. 4, 509-522.

[10] A. C. Berg, T. L. Berg e J. Malik, Shape matching and object recognition using low distortion correspondences, IEEE Conf. on Computer Vision and Pattern Recognition (Washington, DC, USA), vol. 1, IEEE Computer Society, 2005, pp. 26-33. 
[11] S. Berretti, A. Del Bimbo e E. Vicario, Efficient matching and indexing of graph models in content-based retrieval, IEEE Trans. Pattern Anal. Mach. Intell. 23 (2001), 1089-1105.

[12] J. Besag, Spatial interaction and the statistical analysis of lattice systems, Journal of the Royal Statistical Society, Series B 36 (1974), no. 2, 192-236.

[13] P. J. Besl e H. D. McKay, A method for registration of 3-d shapes, IEEE Trans. Pattern Anal. Mach. Intell. 14 (1992), no. 2, 239-256.

[14] H. Bezerra, B. Feijo e L. Velho, A computer-assisted colorization algorithm based on topological difference, Proc. Brazilian Symposium on Computer Graphics and Image Processing (Los Alamitos) (C. Jung e M. Walter, eds.), IEEE Computer Society, Oct. 2006, pp. 71-77.

[15] Hedlena Bezerra, Colorização $3 d$ para animação 2d, Master's thesis, Pontifícia Universidade Católica do Rio de Janeiro, May 2005.

[16] A. Blake, C. Rother, M. Brown e P. Torr, Interactive image segmentation using an adaptive gmmrf model, Proc. of the European Conference on Computer Vision, LNCS, no. 3021, Springer-Verlag, 2004, pp. 428-441.

[17] Y. Boykov e M. Jolly, Interactive graph cuts for optimal boundary and region segmentation of objects in n-d images, In Proc. IEEE Intl. Conf. on Computer Vision 1 (2001), 105-112.

[18] Y. Boykov, O. Veksler e R. Zabih, Fast approximate energy minimization via graph cuts, IEEE Trans. Pattern Anal. Mach. Intell. 23 (2001), no. 11, 1222-1239.

[19] N. Burtnyk e M. Wein, Computer-Generated Key Frame Animation, Journal of the Society of Motion Picture and Television Engineers 80 (1971), no. 3, 149-153.

[20] T. Caelli e T. Caetano, Graphical models for graph matching: approximate models and optimal algorithms, Pattern Recognition Letters 26 (2005), no. 3, 339-346.

[21] T. Caetano, Graphical models and point set matching, Ph.D. thesis, UFRGS, Porto Alegre, RS, BRA, 2004, Advisor: D. A. C. Barone, Coadvisor: T. Caelli.

[22] T. S. Caetano, J. J. McAuley, L. Cheng, Q. V. Le e A. J. Smola, Learning graph matching, IEEE Trans. Pattern Anal. Mach. Intell. 31 (2009), no. 6, 1048-1058.

[23] T. S. Caetano, D. Schuurmans e D. A. C. Barone, Graphical models and point pattern matching, IEEE Trans. Pattern Anal. Mach. Intell. 28 (2006), no. 10, 1646-1663. 
[24] M. Carcassoni e E. R. Hancock, Spectral correspondence for point pattern matching, Pattern Recognition 36 (2003), no. 1, 193-204.

[25] E. Catmull, The problems of computer-assisted animation, ACM SIGGRAPH 1978 papers (New York, NY, USA), ACM, 1978, pp. 348-353.

[26] R. M. Cesar-Jr., E. Bengoetxea, I. Bloch e P. Larrañaga, Inexact graph matching for model-based recognition: evaluation and comparison of optimization algorithms, Pattern Recognition 38 (2005), no. 11, 2099-2113.

[27] L. Chen, J. J. McAuley., R. S. Feris, T. S. Caetano e M. Turk, Shape classification through structured learning of matching measures, IEEE Conf. on Computer Vision and Pattern Recognition, June 2009, pp. 365-372.

[28] W. J. Christmas, J. Kittler e M. Petrou, Structural matching in computer vision using probabilistic relaxation, IEEE Trans. Pattern Anal. Mach. Intell. 17 (1995), no. 8, 749764.

[29] H. Chui e A. Rangarajan, A new algorithm for non-rigid point matching, IEEE Conf. on Computer Vision and Pattern Recognition 2 (2000), 44-51.

[30] _ A new point matching algorithm for non-rigid registration, Comput. Vis. Image Underst. 89 (2003), no. 2-3, 114-141.

[31] L. A. Consularo, R. M. Cesar-Jr. e I. Bloch, Structural image segmentation with interactive model generation, Proc. IEEE Intl. Conf. on Image Processing, IEEE, Piscataway, NJ, 2007.

[32] D. Conte, P. Foggia, C. Sansone e M. Vento, Thirty years of graph matching in pattern recognition, Intl. Journal of Pattern Recognition and Artificial Intelligence 18 (2004), no. 3, 265-298.

[33] T. Cour, P. Srinivasan e J. Shi, Balanced graph matching, Advances in Neural Information Processing Systems (Cambridge, MA) (B. Schölkopf, J. Platt e T. Hoffman, eds.), MIT Press, 2007, pp. 313-320.

[34] E. R. Dougherty e R. A. Lotufo, Hands-on morphological image processing, SPIE PRESS, 2003.

[35] O. Duchenne, J.-Y. Audibert, R. Keriven, J. Ponce e F. Segonne, Segmentation by transduction, IEEE Conf. on Computer Vision and Pattern Recognition, June 2008, pp. 1-8. 
[36] J. Duchi, D. Tarlow, G. Elidan e D. Koller, Using combinatorial optimization within maxproduct belief propagation, Advances in Neural Information Processing Systems, 2007.

[37] M. Eshera e K. S. Fu, A graph distance measure for image analysis, IEEE Trans. on Systems, Man, and Cybernetics 14 (1984), no. 3, 353-363.

[38] P. F. Felzenszwalb, Representation and detection of deformable shapes, IEEE Trans. Pattern Anal. Mach. Intell. 27 (2005), no. 2, 208-220.

[39] P. F. Felzenszwalb e D. P. Huttenlocher, Efficient belief propagation for early vision, Intl. J. Comput. Vision 70 (2006), no. 1, 41-54.

[40] M. A. Fischler e R. A. Elschlager, The representation and matching of pictorial structures, IEEE Trans. Computers 22 (1973), no. 1, 67-92.

[41] R. Rojas G. Friedland, K. Jantz, Siox: simple interactive object extraction in still images, Proc. of the IEEE Int. Symposium on Multimedia (2005), 253-259.

[42] F. Ge, S. Wang e T. Liu, New benchmark for image segmentation evaluation, Journal of Electronic Imaging 16 (2007), no. 3.

[43] S. Geman e D. Geman, Stochastic relaxation, Gibbs distributions, and the Bayesian restoration of images, Readings in uncertain reasoning (1990), 452-472.

[44] S. Gold e A. Rangarajan, A graduated assignment algorithm for graph matching, IEEE Trans. Pattern Anal. Mach. Intell. 18 (1996), no. 4, 377-388.

[45] A. B. V. Graciano, A. Noma, L. A. Consularo, R. M. Cesar-Jr e I. Bloch, Inexact graph matching for segmentation and recognition of object parts, Tech. report, Institute of Mathematics and Statistics, University of Sao Paulo, Brazil, 2009.

[46] L. Grady, Random walks for image segmentation, IEEE Trans. Pattern Anal. Mach. Intell. 28 (2006), no. 11.

[47] E. R. Hancock e R. C. Wilson, Graph-based methods for vision: a yorkist manifesto, Proceedings of the Joint IAPR International Workshop on Structural, Syntactic, and Statistical Pattern Recognition (London, UK), Springer-Verlag, 2002, pp. 31-46.

[48] B. W. Hong, E. Prados, S. Soatto e L. Vese, Shape representation based on integral kernels: application to image matching and segmentation, IEEE Conf. on Computer Vision and Pattern Recognition, vol. 1, IEEE, June 2006, pp. 833-840. 
[49] J. V. Kittler e E. R. Hancock, Combining evidence in probabilistic relaxation, Intl. Journal of Pattern Recognition and Artificial Intelligence 3 (1989), 29-51.

[50] V. Kolmogorov e R. Zabih, What energy functions can be minimized via graph cuts?, IEEE Trans. Pattern Anal. Mach. Intell. 26 (2004), no. 2, 147-159.

[51] L. J. Latecki, R. Lakamper e U. Eckhardt, Shape descriptors for non-rigid shapes with a single closed contour, IEEE Conf. on Computer Vision and Pattern Recognition 1 (2000), 1424.

[52] Y. Lecun, L. Bottou, Y. Bengio e P. Haffner, Gradient-based learning applied to document recognition, Proc. of the IEEE, 1998, pp. 2278-2324.

[53] M. Leordeanu e M. Hebert, A spectral technique for correspondence problems using pairwise constraints, In Proc. IEEE Intl. Conf. on Computer Vision (Washington, DC, USA), IEEE Computer Society, 2005, pp. 1482-1489.

[54] S. Z. Li, Markov random field modeling in computer vision, Springer-Verlag New York, Inc., Secaucus, NJ, USA, 1995.

[55] Y. Li, J. Sun, C.-K. Tang e H.-Y. Shum, Lazy snapping, ACM Trans. Graph. 23 (2004), no. $3,303-308$.

[56] H. Ling e D. W. Jacobs, Shape classification using the inner-distance, IEEE Trans. Pattern Anal. Mach. Intell. 29 (2007), no. 2, 286-299.

[57] B. Luo e E. R. Hancock, Structural graph matching using the em algorithm and singular value decomposition, IEEE Trans. Pattern Anal. Mach. Intell. 23 (2001), no. 10, 11201136.

[58] J. S. Madeira, André Stork e M. H. Gross, An approach to computer-supported cartooning, The Visual Computer 12 (1996), no. 1, 1-17.

[59] D. Martin, C. Fowlkes, D. Tal e J. Malik, A database of human segmented natural images and its application to evaluating segmentation algorithms and measuring ecological statistics, In Proc. IEEE Intl. Conf. on Computer Vision, vol. 2, July 2001, pp. 416-423.

[60] K. McGuinness e N. E. O'Connor, A comparative evaluation of interactive segmentation algorithms, IEEE Transactions on Image Processing 43 (2010), no. 2, 434-444.

[61] H. Murase e S. K. Nayar, Visual learning and recognition of 3-d objects from appearance, Intl. J. Comput. Vision 14 (1995), no. 1, 5-24. 
[62] J. Ning, L. Zhang, D. Zhang e C. Wu, Interactive image segmentation by maximal similarity based region merging, IEEE Transactions on Image Processing 43 (2010), no. 2, 445-456.

[63] A. Noma e R. M. Cesar-Jr, Sparse representantions for efficient shape matching, Proc. Brazilian Symposium on Computer Graphics and Image Processing, IEEE Computer Society, 2010.

[64] A. Noma, A.B.V. Graciano, L.A. Consularo, R.M. Cesar-Jr e I. Bloch, A new algorithm for interactive structural image segmentation, arXiv:0805.1854v2 [cs.CV], 2008.

[65] A. Noma, A. Pardo e R. M. Cesar-Jr, Structural matching of 2d electrophoresis gels using deformed graphs, Pattern Recognition Letters (Accepted) (2010).

[66] A. Noma, A. Pardo e R.M. Cesar-Jr, Structural matching of 2D electrophoresis gels using graph models, Proc. Brazilian Symposium on Computer Graphics and Image Processing (Los Alamitos) (C. Jung e M. Walter, eds.), IEEE Computer Society, Oct. 12-15 2008.

[67] A. Noma, L. Velho e R. M. Cesar-Jr, A computer-assisted colorization approach based on efficient belief propagation and graph matching, CIARP '09: Proceedings of the 14th Iberoamerican Conference on Pattern Recognition (Berlin, Heidelberg), Springer-Verlag, 2009, pp. 345-352.

[68] C. H. Papadimitriou e K. Steiglitz, Combinatorial optimization: algorithms and complexity, Prentice-Hall, Inc., Upper Saddle River, NJ, USA, 1982.

[69] _ Combinatorial optimization: algorithms and complexity, Prentice-Hall, 1998.

[70] J. Pearl, Probabilistic reasoning in intelligent systems: networks of plausible inference, Morgan Kaufmann, 1988.

[71] B. L. Price, B. Morse e S. Cohen, Geodesic graph cut for interactive image segmentation, IEEE Conf. on Computer Vision and Pattern Recognition, 2010.

[72] A. Protiere e G. Sapiro, Interactive image segmentation via adaptive weighted distances, IEEE Transactions on Image Processing 16 (2007), no. 4, 1046-1057.

[73] J. Qiu, H. S. Seah, F. Tian, Q. Chen e K. Melikhov, Computer-assisted auto coloring by region matching, $P G$ '03: Proceedings of the 11th Pacific Conference on Computer Graphics and Applications (Washington, DC, USA), IEEE Computer Society, 2003, p. 175.

[74] M. Rogers e M. Graham, Robust and accurate registration of 2-d electrophoresis gels using point matching, IEEE Transactions on Image Processing 16 (2007), no. 3, 624-635. 
[75] A. Rosenfeld, R. A. Hummel e S. W. Zucker, Scene labeling by relaxation operations, IEEE Trans. on Systems, Man, and Cybernetics 6 (1976), no. 6, 420-433.

[76] C. Rother, V. Kolmogorov e A. Blake, "grabcut": interactive foreground extraction using iterated graph cuts, ACM Trans. Graph. 23 (2004), no. 3, 309-314.

[77] A. Sanfeliu e K. S. Fu, A distance measure between attributed relational graphs for pattern recognition, IEEE Trans. on Systems, Man, and Cybernetics 13 (1983), no. 3, 353-362.

[78] C. Schellewald e C. Schnörr, Probabilistic subgraph matching based on convex relaxation, EMMCVPR, 2005, pp. 171-186.

[79] H. S. Seah e F. Tian, Computer-assisted coloring by matching line drawings, The Visual Computer 16 (2000), no. 5, 289-304.

[80] L. G. Shapiro e R. M. Haralick, Structural descriptions and inexact matching, IEEE Trans. Pattern Anal. Mach. Intell. 3 (1981), no. 5, 504-519.

[81] _ A metric for comparing relational descriptions, IEEE Trans. Pattern Anal. Mach. Intell. 7 (1985), no. 1, 90-94.

[82] D. Sharvit, J. Chan, H. Tek e B. Kimia, Symmetry-based indexing of image databases, J. Visual Comm. and Image Representation 9 (1998), 366-380.

[83] S. Todorovic e N. Ahuja, Region-based hierarchical image matching, Int. J. Comput. Vision 78 (2008), no. 1, 47-66.

[84] _ Unsupervised category modeling, recognition, and segmentation in images, IEEE Trans. Pattern Anal. Mach. Intell. 30 (2008), no. 12, 2158-2174.

[85] P. H. S. Torr, Solving Markov random fields using semi definite programming, 9th International Workshop on Artificial Intelligence and Statistics, 2003.

[86] L. Torresani, V. Kolmogorov e C. Rother, Feature correspondence via graph matching: models and global optimization, Proc. of the European Conference on Computer Vision (Berlin, Heidelberg), Springer-Verlag, 2008, pp. 596-609.

[87] A. Torsello e E. R. Hancock, Computing approximate tree edit distance using relaxation labeling, Pattern Recognition Letters 24 (2003), no. 8, 1089-1097.

[88] W. H. Tsai e K. S. Fu, Error-correcting isomorphisms of attributed relational graphs for pattern analysis, IEEE Trans. on Systems, Man, and Cybernetics 9 (1979), no. 12, 757-768. 
[89] _ Subgraph error-correcting isomorphisms for syntactic pattern recognition, IEEE Trans. on Systems, Man, and Cybernetics 13 (1983), no. 1, 48-62.

[90] S. Umeyama, An eigen decomposition approach to weighted graph matching problems, IEEE Trans. Pattern Anal. Mach. Intell. 10 (1988), 695-703.

[91] M. van Eede, D. Macrini, A. Telea, C. Sminchisescu e S.Dickinson, Canonical skeletons for shape matching, Proc. of the Int. Conf. on Pattern Recognition (Washington, DC, USA), IEEE Computer Society, 2006, pp. 64-69.

[92] O. Veksler, Efficient graph-based energy minimization methods in computer vision, Ph.D. thesis, Cornell University, Ithaca, NY, USA, 1999, Adviser-Ramin Zabih.

[93] L. Vincent e P. Soille, Watersheds in digital spaces: an efficient algorithm based on immersion simulations, IEEE Trans. Pattern Anal. Mach. Intell. 13 (1991), no. 6, 583-598.

[94] Y. Weiss, Correctness of local probability propagation in graphical models with loops, Neural Comput. 12 (2000), no. 1, 1-41.

[95] Y. Weiss e W. T. Freeman, On the optimality of solutions of the max-product beliefpropagation algorithm in arbitrary graphs, IEEE Transactions on Information Theory $\mathbf{4 7}$ (2001), no. 2, 736-744.

[96] C. Yang, R. Duraiswami, N. A. Gumerov e L. Davis, Improved fast gauss transform and efficient kernel density estimation, In Proc. IEEE Intl. Conf. on Computer Vision (Washington, DC, USA), IEEE Computer Society, 2003, p. 464.

[97] J. S. Yedidia, W. T. Freeman e Y. Weiss, Understanding belief propagation and its generalizations, Exploring artificial intelligence in the new millennium (2003), 239-269. 